\title{
Dealing with Multiple Perceptions of 'Reality': Change within a Transorganizational System
}

\author{
Michael Halinski, PhD Candidate \\ Sprott School of Business, Carleton University \\ A thesis proposal submitted to the Faculty of Graduate and \\ Postdoctoral Affairs in partial fulfillment of the requirements \\ for the degree of Doctor of Philosophy, Management \\ Eric Sprott School of Business \\ Carleton University \\ Ottawa, Ontario
}




\begin{abstract}
Transorganizational systems (TSs) are defined as "coalition structures formed by two or more organizations for a common purpose" (Cummings, Blumenthal, \& Greiner, 1983; p. 379). Although an extensive body of knowledge exists on TS outcomes, there has been little research exploring how TSs change over time.

The thesis presents an exploratory, longitudinal, multi-method case study of a change initiative within a TS consisting of a police organization, a hospital, and a social service agency operating in a large city in Canada. The initiative involved the change to a process that is interdependently co-owned by all three organizations and relates to how these three partners deal with persons suspected of having mental illness in the community. Seventy-five semi-structured interviews ( 25 per organization) were conducted before and sixty semi-structured interviews (20 per organization) were conducted six months after the implementation of the planned TS change. The data from these interviews were supplemented with archival data from the police.

We draw from planned change (e.g. Burnes, 2004), TS (e.g. Crosby, Bryson, \& Stone, 2006), and stakeholder (e.g. Mitchel, Agle, \& Wood, 1997) literatures to analyze findings from this thesis. The results of this thesis indicate that the planned TS change has had a measurable impact on the TS. The results also suggest that respondents' perceptions of what they wanted to change (pre-change) and what they observed had changed (post-change) in the TS influenced how they made sense of the planned TS change, and that these sensemaking processes affected their views of interorganizational relationships. Our analysis also suggests that the planned TS change: (1) shifted power within the TS from the hospital to the police, (2) shifted urgency within the TS from the police to the hospital, and (3) eroded the legitimacy of the police in the eyes of healthcare workers.
\end{abstract}




\section{ACKNOWLEDGEMENTS}

This research was not completed by a single individual alone. Many people contributed to development and evolution of this dissertation. First, and foremost, I would like to thank my supervisor, Dr. Linda Duxbury. Throughout the process of conducting this research, she has taught me as much about myself as she has about becoming an academic. I will never forget our journey over the past few years - thank you.

I would also like to thank my committee members: Dr. David Cray, Dr. Michel Rod, Dr. Craig Bennel, and Dr. Raymond Paquin. Your insight and guidance has been critical to the development of this dissertation.

I would also like to thank the respondents who participated in this study. Without the insights provided by police officers, healthcare workers, and community support workers, this research could not have been conducted. In particular, I would like to thank the Deputy Chief of the police service and the Executive Director of the police board who initially came up to visit Linda and I three years ago. Without their commitment to this research, this research would have never begun.

Lastly, this dissertation could not have been completed without the support, encouragement, patience, and motivation of my friends and family. Thank you Greg Dole, Christopher Smith, and Dr. Laura Gover, as you have provided instrumental and emotional support through this lengthy and challenging endeavour. Thank you to my mom, Diane Halinski, as without your support and optimism I may not have been able to finish. And, most importantly, I extend my gratitude and love to the most important person in the world, Dr. Vanessa LaSalle, to whom I dedicate this thesis. 


\section{TABLE OF CONTENTS}

CHAPTER 1: INTRODUCTION................................................................. 1

1.1 FRAMING THE DISSERTATION RESEARCH ....................................5

1.2 RESEARCH CONTRIBUTIONS ...............................................................6 6

1.3 ORGANIZATION OF THE DISSERTATION ............................................ 7

CHAPTER 2: LITERATURE REVIEW ............................................ 9

2.1 THE NATURE OF PLANNED CHANGE ...................................................... 10

2.1.1 Approaches to Studying Change ......................................................... 10

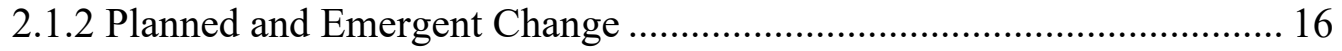

2.1.3 Lewin's Approach to Planned Change................................................ 20

2.1.4 Factors Affecting Planned Change ....................................................... 30

2.1.5 Critique of Planned Organizational Change Literature........................... 37

2.2 TRANSORGANIZATIONAL SYSTEM (TS) CHANGE ........................... 40

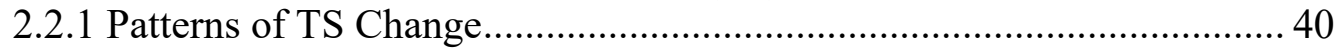

2.2.2 Factors Driving and Shaping TS Change........................................ 42

2.2.3 TS Change in the Public Sector ......................................................... 47

2.2.4 Critique of TS Change Literature ...................................................... 47

2.3 STAKEHOLDER THEORY ............................................................................ 48

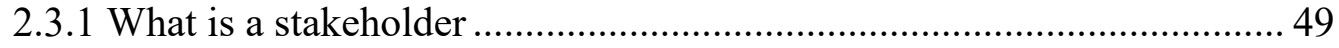

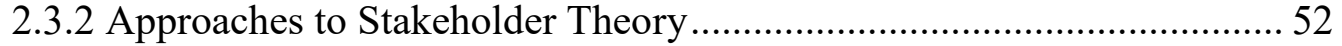

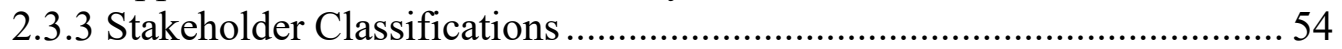

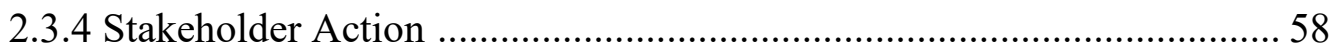

2.3.5 Critique of Stakeholder Literature ..................................................... 62

\section{CHAPTER 3: DEVELOPMENT OF RESEARCH OBJECTIVES}

AND QUESTIONS .............................................................................. 65

3.1 RESEARCH OBJECTIVES............................................................................6 65

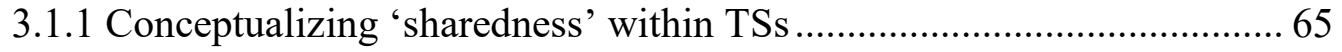

3.1.2 Conceptualizing Transorganizational Change .................................... 68

3.2 RESEARCH QUESTIONS ............................................................................ 74

3.2.1 RESERCH OBJECTIVE ONE: Develop a framework describing how a planned TS change impacts perceptions of TS stakeholder relationships over time

3.2.2 RESEARCH OBJECTIVE TWO: Does the 'sharedness' concept provide a useful lens through which to understand the impact of planned TS change on perceptions of TS stakeholder relationships ........ 75 
CHAPTER 4: RESEARCH DESIGN ................................................. 77

4.1 CASE STUDY METHODOLOGY ............................................................... 78

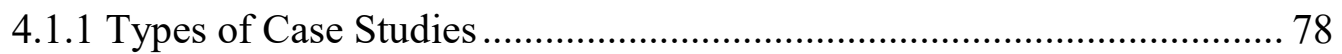

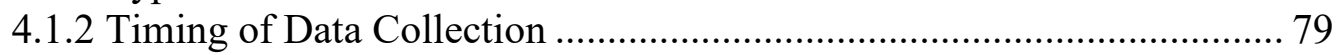

4.1.3 Single- and Multiple-Case Case Studies ............................................... 80

4.1.4 Multi-Method Case Studies ................................................................. 81

4.1.5 Case Study Approaches ..................................................................... 82

4.1.6 Inductive Case Study Roadmap ……………………………………..... 83

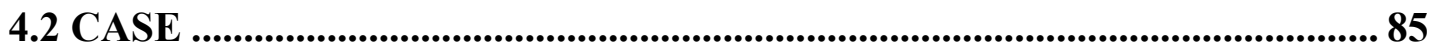

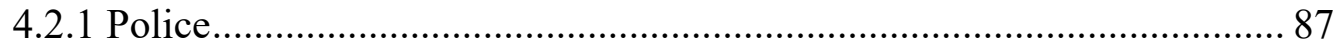

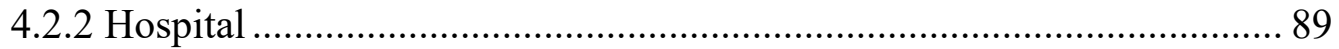

4.2.3 Social Service Agency ........................................................................ 91

4.2.4 Environmental Shifts Impacting the PMI Process .................................. 92

4.2.5 Situation leading up to the Planned TS Change....................................... 94

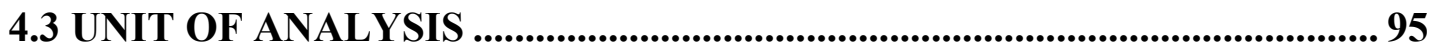

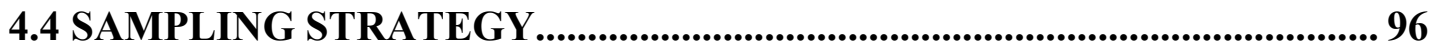

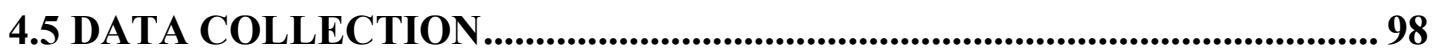

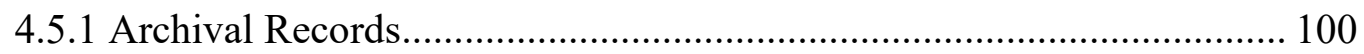

4.5.2 Semi-Structured Interviews................................................................. 101

4.6 INTERACTIONAL EXPERTISE ................................................................. 108

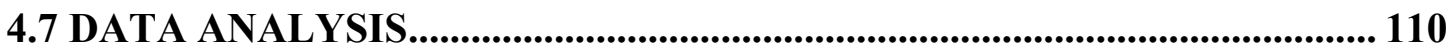

4.7.1 Case Based Qualitative Data Analysis .................................................. 111

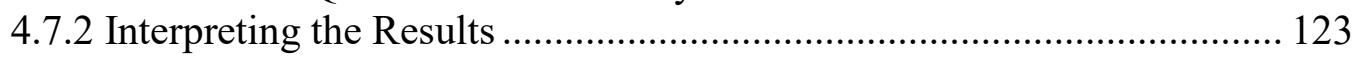

4.7.3 Triangulation ................................................................................... 125

4.8 METHODOLOGY IN PRACTICE............................................................... 126

4.8.1 Description of Time 1 Sample ………………................................. 127

4.8.2 Description of Time 2 Sample ……………………............................. 128

\section{CHAPTER 5: ANALYSIS OF THE RESULTS THROUGH A} CHANGE MANAGEMENT LENS ............................................... 134

5.1 WHAT CHANGED: THE REALITY OF THE PLANNED TS CHANGE . 134

5.1.1 Kick-Off Meeting........................................................................... 135

5.1.2 Specific Changes to be Implemented..................................................... 137

5.2 WHAT CHANGED: AN OBJECTIVE ANALYSIS OF THE PLANNED TS

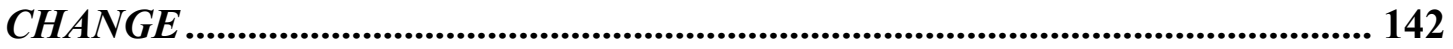

5.2.1 Percentage of PMIs Apprehended by Police per Month.......................... 143

5.2.2 Percentage of EDP Forms Completed by Police per Month.................... 144

5.2.3 Percentage of PMIs Apprehended by Police and Formed by a

Physician per Month ............................................................................ 145

5.2.4 Average Length of Time PMIs Apprehended by Police have to

Wait to be Assessed by a Physician per month.......................................... 146

5.2.5 Key Findings from Analysis of Change Indicators.................................. 147 
5.3 WHAT CHANGED: A PERCEPTUAL ANALYSIS OF THE PLANNED TS CHANGE

5.3.1 Perceptions of Desired Changes within the TS: Total Sample

Pre-Change (Time 1).

5.3.2 Perceptions of Desired Changes within the TS: TS Stakeholder

Sample Pre-Change (Time 1)

5.3.3 Perceptions of What has Changed in the TS: Total Sample Post-

Change (Time 2)

5.3.4 Perceptions of What has Changed in the TS: TS Stakeholder Sample

Post-Change (Time 2)

5.3.5 Perceptions of Desired and Perceived Changes within the TS:

Analysis of the Cross-Sectional Data Set

5.4 WHAT CHANGED: A SENSEMAKING ANALYSIS OF THE

PLANNED TS CHANGE 178

5.4.1 Sensemaking about Planned TS Change

5.4.2 Theoretical Development of a Typology: Making Sense of Planned

TS Change

5.4.3 Methodology: Making sense of Planned TS Change.

5.4.4 Empirical Assessment of the Making Sense of Planned TS Change:

Within-case Analysis 186

5.4.5 Empirical Assessment of the Making Sense of Planned TS Change:

Cross-Case Analysis

5.5 DISCUSSION: PLANNED TS CHANGE

5.5.1 Management Actions Impacted How Change Unfolded in the

TS over time 224

5.5.2 Desired Changes within the TS

5.5.3 Perceived Changes within the TS 227

5.5.4 You Can't Always Get What You Want................................................. 228

5.5.5 Making Sense of Planned TS Change................................................... 229

\section{CHAPTER 6: ANALYSIS OF THE RESULTS THROUGH A}

STAKEHOLDER THEORY LENS

6.1 PERCEPTIONS OF POWER WITHIN THE TS........................................... 232

6.1.1 Perceptions of Power within the TS: Total Sample Pre-Change ........... 233

6.1.2 Perceptions of Power within the TS: TS Stakeholder Sample

Pre-Change 236

6.1.3 Perceptions of Power within the TS: Total Sample Post-Change............ 240

6.1.4 Perceptions of Power within the TS: TS Stakeholder Sample

Post-Change 244

6.1.5 Impact of Planned TS Change on Perceptions of Power within the TS . 247

6.2 PERCEPTIONS OF LEGITIMACY WITHIN THE TS............................. 261

6.2.1 Perceptions of Legitimacy within the TS: Total Sample Pre-Change .... 261

6.2.2 Perceptions of Legitimacy within the TS: TS Stakeholder Sample

Pre-Change 267

6.2.3 Perceptions of Legitimacy within the TS: Total Sample Post-Change... 271 
6.2.4 Perceptions of Legitimacy within the TS: TS Stakeholder Sample

Post-Change

6.2.5 Impact of Planned TS Change on Perceptions of Legitimacy within the TS

6.3 PERCEPTIONS OF URGENCY WITHIN THE TS 293

6.3.1 Perceptions of Urgency within the TS: Total Sample Pre-Change. 293

6.3.2 Perceptions of Urgency within the TS: TS Stakeholder Sample

Pre-Change 298

6.3.3 Perceptions of Urgency within the TS: Total Sample Post-Change ....... 301

6.3.4 Perceptions of Urgency within the TS: TS Stakeholder Sample

Post-Change 306

6.3.5 Impact of Planned TS Change on Perceptions of Urgency within the TS

6.4 DISCUSSION: IMPACT OF PLANNED TS CHANGE ON

PERCEPTIONS OF TS STAKEHOLDER RELATIONSHIPS

6.4.1 DISCUSSION: Perceptions of Power within the TS .............................. 324

6.4.2 DISCUSSION: Perceptions of Legitimacy within the TS ....................... 330

6.4.3 DISCUSSION: Perceptions of Urgency within the TS ........................... 336

CHAPTER 7: CONCLUSIONS

7.1 THE UTILITY OF THE 'SHAREDNESS' CONCEPT FOR STUDYING PLANNED TS CHANGE

7.2 DEVELOP A FRAMEWORK DESCRIBING HOW A PLANNED TS CHANGE IMPACTS PERCEPTIONS OF TS STAKEHOLDER RELATIONSHIPS OVER TIME

7.2.1 Perceptions of TS Stakeholder Relationships

7.2.2 Perceptions of Desired TS Change and Perceptions of What Has

Changed in the TS 360

7.2.3 Making Sense of Planned TS Change Typology 368

7.2.4 Relationships Between Constructs in the Refined Theoretical Framework

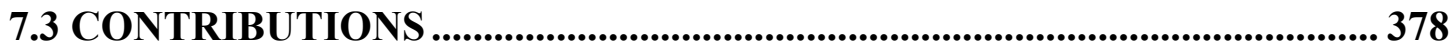

7.4 LIMITATIONS AND DIRECTIONS FOR FUTURE RESEARCH ............ 379

CHAPTER 8: REFERENCES 


\section{LIST OF TABLES}

Table 1: Comparison of Variance and Process Approaches

(Poole et al., 2000)

Table 2: Summary of Factors Affecting Planned Change

Table 3: Steps for Building Theory from Case Study

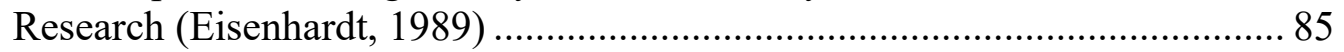

Table 4: Mapping interview questions to Orienting Framework ......................... 103

Table 5: Example of developing the coding scheme ...................................... 113

Table 6: Example of first table type used to display results ............................... 116

Table 7: Example of second table type used to display results........................... 118

Table 8: Example of third table type used to display results ............................... 121

Table 9: Example of fourth table type used to display results ............................. 123

Table 10: Time 1 Interviewee Demographics................................................. 127

Table 11: Demographics of respondents interviewed for a second time .............. 130

Table 12: Demographics of Social Service Agency employees by time

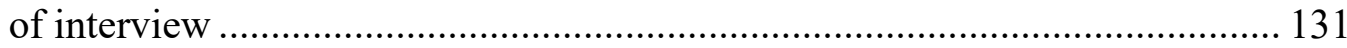

Table 13: Summary of interviews carried out.................................................. 132

Table 14: Mapping decision-makers' objectives to implemented changes and indicators used to evaluate change.

Table 15: Percent of PMIs apprehended by Police per month: Sept. 2014 to May 2015

Table 16: Percent of EDP forms completed by Police per month: May 2014 to May 2015

Table 17: Percent of PMIs apprehended by Police and formed by a physician: Sept. 2013 to May 2015 .

Table 18: Average number of hours PMIs apprehended by Police have to wait to be assessed by a doctor: Sept. 2013 to May 2015

Table 19: Decision-Makers' Objectives and Change Outcomes

Table 20: Perceptions of Desired Changes in the TS: Total Sample Pre-Change

Table 21: Perceptions of Desired Changes in the TS: TS Stakeholder Sample Pre-Change

Table 22: Perceptions of What Has Changed in the TS: Total Sample Post-Change

Table 23: Perceptions of What Has Changed in the TS: TS Stakeholder Sample Post-Change

Table 24: Comparison of Perceptions of Desired and Perceived Changes in the TS

Table 25: Weick's (1995) Seven Properties of Sensemaking.............................. 181

Table 26: Typology of Making Sense of Planned TS change ............................. 182

Table 27: Making Sense of Improved Interorganizational Communication.......... 188

Table 28: Got Desired Change: Increased Interorganizational Communication ... 189

Table 29: Unanticipated Change: Increased Interorganizational Communication 190 
Table 30: Making Sense of Perceptions of an ED Process that Expedites Service for PMIs

Table 31: Got Desired Change: An ED Process that Expedites Service for PMIs 194

Table 32: Thwarted Desires: An ED Process that Expedites Service for PMIs .... 199

Table 33: Unanticipated Change: An ED Process that Expedites Service for

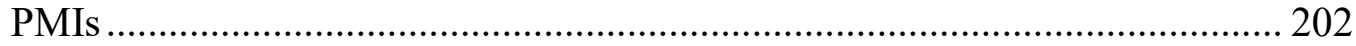

Table 34: Making Sense of Perceptions of Improved MHESU Engagement........ 206

Table 35: Got Desired Change: Improved MHESU Engagement .......................... 207

Table 36: Thwarted Desires: Improved MHESU Engagement ........................... 208

Table 37: Making Sense of Perceptions of Mandatory Completion of EDP

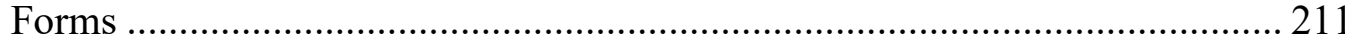

Table 38: Thwarted Desires: Mandatory Completion of EDP Forms .................. 213

Table 39: Unanticipated Change: Mandatory Completion of EDP Forms ........... 214

Table 40: Making Sense of Planned TS change .................................................... 217

Table 41: Perceptions of Power within the TS: Total Sample Pre-Change .......... 233

Table 42: Perceptions of Power within the TS: TS Stakeholder

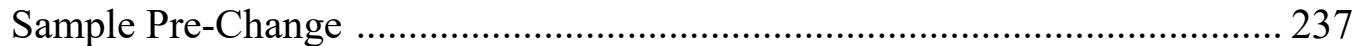

Table 43: Perceptions of Power within the TS: Total Sample Post-Change ........ 241

Table 44: Perceptions of Power within the TS: TS Stakeholder Sample Pre-Change

Table 45: Perceptions of Power within the TS Over Time: Cross-Sectional Data.

Table 46: Perceptions of Power within the TS Over Time: Representative Panel Data

Table 47: Perceptions of Legitimacy within the TS: Total Sample Pre-Change

Table 48: Perceptions of Legitimacy within the TS: TS Stakeholder Sample Pre-Change

Table 49: Perceptions of Legitimacy within the TS: Total Sample Post-Change

Table 50: Perceptions of Legitimacy within the TS: TS Stakeholder Sample Pre-Change 280

Table 51: Perceptions of Legitimacy within the TS Over Time: Cross-Sectional Data...

Table 52: Perceptions of Legitimacy within the TS Over Time: Representative Panel Data ......................................................................... 288

Table 53: Perceptions of Urgency within the TS: Total Sample Pre-Change ...... 294

Table 54: Perceptions of Urgency within the TS: TS Stakeholder Sample Pre-Change

Table 55: Perceptions of Urgency within the TS: Total Sample Post-Change

Table 56: Perceptions of Urgency within the TS: TS Stakeholder Sample Pre-Change

Table 57: Perceptions of Urgency within the TS Over Time: Cross-Sectional Data. 
Table 58: Perceptions of Urgency within the TS Over Time:

Representative Panel Data ........................................................................ 317

Table 59: Comparison of what TS stakeholders mentioned they wanted and

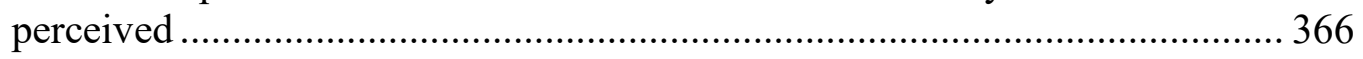




\section{LIST OF FIGURES}

Figure 1: Approaches to Studying Change (Van de Ven \& Poole, 2005) ............. 15

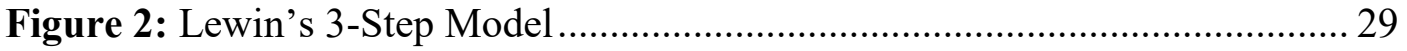

Figure 3: Degree of Organization within TSs (Roberts, 2004) .......................... 45

Figure 4: Frooman's (1999) Typology of Influence Strategies ............................ 59

Figure 5: Conceptualizing 'sharedness' with Common, Shared, and Unique

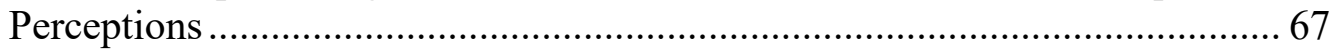

Figure 6: Orienting Framework guiding this study of how a planned TS change impacts perceptions of TS Stakeholder Relationships over time

Figure 7: Decision tree used classify first-order codes based on 'sharedness' construct.....

Figure 8: Timeline of case study research

Figure 9: Example of Venn diagram used to summarize key findings regarding 'sharedness'.

Figure 10: Revisiting the preliminary conceptual framework..... 346

Figure 11: Refined Theoretical Framework of how planned TS change impacts

TS stakeholder relationships over time

Figure 12: Summary of Perceptions of Power within the TS: Pre- and Post-Change 352

Figure 13: Summary of Perceptions of Legitimacy within the TS: Pre- and Post-Change 354

Figure 14: Summary of Perceptions of Urgency within the TS: Pre- and Post-Change 356

Figure 15: Summary of Perceptions of Desired and Perceived Changes within the TS: Pre- and Post-Change. 


\section{LIST OF APPENDICES}

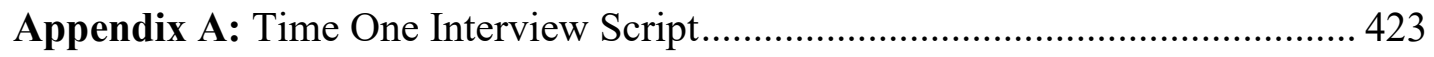

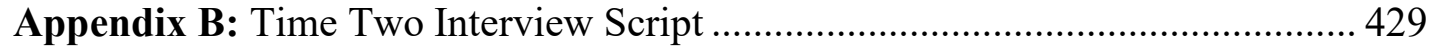

Appendix C: Complete Panel Data Displays..................................................... 434

Appendix D: Data Displays for Intra-Organizational Analysis........................... 438 


\section{CHAPTER 1: INTRODUCTION}

During the past several decades, organizations have experienced significant shifts in their competitive landscape and operating environments (Greenwood \& Hinings, 1996) that have the potential to impact how both researchers and practitioners conceptualize change management. External environmental changes such as increased competition (Goodstein \& Burke, 1991; Kanter, 1991), shifting socio-political environments (Greenwood \& Hinings, 1996), a variable legal landscape (Ivancevich \& Matteson, 2002), rapid technological innovation (Morrison, 1998; Kemelgor, Johnson \& Srinivasan, 2000; Ivancevich \& Matteson, 2002; Kanter, 1991), and dynamic demographic shifts (Senge, 2000; Calo, 2005) have contributed to an increased rate of organizational change (Checa, Maguire \& Barney, 2003). Rossi (2006) notes that for many organizations, these environmental shifts have meant that continuous change is now the norm. Change theoreticians have responded to the above trends by undertaking research which examines 'what changes in organizations' (i.e. content), 'who influences organizational change' (i.e. context), and 'how organizational change unfolds over time' (i.e. process) (Armenakis \& Bedeian, 1999).

The majority of the research in the change management domain relies on models and techniques that were originally conceptualized for the study of change within a single organization (e.g., Judson, 1991; Kotter, 1995; Galpin, 1996; Armenakis, Harris and Field, 1999). Much less is known about change within transorganizational systems (TSs). TSs are defined as "coalition structures formed by two or more organizations for a common purpose" (Cummings, Blumenthal, $\&$ Greiner, 1983; p. 379). This is unfortunate as the extant literature in the management arena reports the need to mitigate organizational decline (Cummings et al., 1983), make sound management decisions (Harrison \& John, 1996), and address the corporate social responsibility 
movement (Porter \& Kramer, 2006) has led to an increase in the number of TSs internationally (Cummings et al., 1983; Cummins \& Worley, 1997; Dyer, Kale \& Singh, 2001; Harper, 1998; Kanter, 1991; Kemelgor et al., 2000; Morrison, 1998). Furthermore, the need to manage change in these unique and complex systems seems to be a challenge faced by virtually all TSs (Bryson, Crosby, \& Stone, 2006).

In the growing yet still small body of research on TS change, scholars have predominantly focused on why TSs change, as opposed to how they change (Ahuja, Soda, \& Zaheer, 2012; Kim, Oh, \& Swaminathan, 2006; Gulati, 1998). More often than not, scholars have focused on changes in interorganizational relationships (i.e., entry into or exit from a TS) as a critical strategic option for organizations attempting to strengthen their capabilities (Hennart, Kim, \& Zeng, 1998; Jones, Hesterly, Fladoe-Lindquist, \& Borgatti, 1998; Das \& Teng, 2000). Much of the previous TS change research has assumed that, if organizations are dissatisfied with partners that do not provide the desired resources, they can (easily) change partners to gain access to such resources from new partners (Kim et al., 2006). This body of research, however, largely ignores theory associated with the embedded nature of interorganizational relationships, which involves stabilizing forces from relationship-specific routines and embedded cultures (Uzzi, 1996). More recently, researchers have argued, using embeddedness logic, that interorganizational relationships play a critical role in how TSs change (Gimeno, 2004; Hagedoorn, 2006). Thus, the primary objective of this thesis is to develop a framework describing how a planned TS change impacts perceptions of interorganizational relationships over time.

'Sharedness' is a new concept to management research that derives from the psychology literature. 'Sharedness', sometimes referred to as social-sharedness, is grounded in two fundamental ideas: (1) groups of individuals can be conceptualized as information-processing 
systems, and (2) the degree to which cognitions, preferences, identities, or perceptions are shared and are being shared within the group affects group dynamics (Tindale \& Kameda, 2000). 'Sharedness' has been traditionally viewed as a lens for analyzing group dynamics within the psychology literature (Kerr et al., 2004). The concept of 'sharedness' has relevance to TSs, as they consist of groups of individuals from different organizations. Despite this fact, we could find little evidence that the 'sharedness' concept has been used to analyze relationships within TSs. Accordingly, the secondary objective of this thesis is to determine the extent to which the 'sharedness' concept provides a useful lens through which to understand the impact of planned TS change on perceptions of interorganizational relationships over time.

To address these research objectives, we undertake a case study that involves a TS consisting of a police organization, a hospital, and a social service agency operating in a large city in Canada. For the purpose of the dissertation research, the scope of our research is limited to a process that is inter-dependently co-owned by all three organizations. This process, which we refer to as the Person suspected of having Mental Illness (PMI) process, relates to how these three partners deal with PMIs in the community. None of the three organizations involved in the PMI process can complete the process on its own. Instead, each organization relies on the unique skillsets of their two partners for task completion. The police manage the initial crises and escort the PMI to the hospital. The hospital psychiatrically assesses and treats the PMI and lets the police know when they can leave the hospital (Canadian law requires the police to stay with the PMI until the hospital retains custody of the PMI). The social service agency contributes ongoing care to the PMI back in the community to minimize the potential for future crises.

The PMI process has recently been impacted by a series of significant changes in the external environment. More specifically, the closure of mental health hospitals and reductions in 
social service funding have increased the number of PMIs that these three organizations have to deal with in a given year and increased the financial and reputational costs of managing the PMI process for all three organizations. These changes to the external environment have, however, differentially impacted the three organizations. The police are spending considerably more time at the hospital waiting for a doctor to assess the PMI (i.e. dependent on hospital resources). During this period, the police are not available to handle other calls in the community (i.e., fewer resources available to provide service to the community). Wait times in the hospital are increasing because of the influx of PMIs brought in by police. Funding for hospitals in the Canadian province, where the study unfolds, are tied to wait times and hospitals with longer wait times may be censored by the Province, see their funding cut, and be the focus of negative coverage by the media. The social service agency is responsible for dealing with PMIs and their families in the community. The PMI, their family, as well as the media have expressed frustration with the status quo and the treatment of those with mental illness by the police and the health care system.

All three of the organizations who were involved in the case study felt that the current PMI process was untenable and desired change. However, the interdependent nature of the relationships between the three organizations in the PMI process suggested that a change resulting in a win for one group (e.g. police are not waiting as long at the hospital) resulted in a loss for another group (e.g. hospital has to add more security costs and overburden their waiting room further). Given the external environment's differential impact on each of the organizations and the fact that a win for one group suggested a loss for another, how might the planned TS change impact perceptions of interorganizational relationships over time? 


\subsection{FRAMING THE DISSERTATION RESEARCH}

This case study involves a unique research opportunity to explore a change in a TS over time. As there is little theoretical or empirical research on change at a level of analysis higher than an organization (Ahuja et al., 2012), there are many different ways to advance theory and methodology in this complex area of research. Accordingly, this section frames this thesis by discussing two critical aspects of the approach taken within this study and two important ways that we restrict the scope of this study.

First, this thesis is grounded in Lewin's seminal research on group dynamics (1943) and planned change (1947a), which focuses on the individual and the collective. We supplement Lewin's research with two more recent theories (i.e., TS, stakeholder theory) that have been developed for higher levels of analysis (i.e., organization, groups of organizations). We use TS theory to explain the unique challenges of collaborating across organizational boundaries (Bryson et al., 2006), whereas we use theory from the stakeholder literature to conceptualize interorganizational relationships within the TS (Mitchell et al., 1997).

Second, this research takes an inductive approach to developing theory from a case study and follows Eisenhardt's (1989) methodology for case study research, which is explained in detail in Chapter 4. Although there is an implicit assumption that theory building from cases is less precise, objective, and rigorous than large-scale hypothesis testing, Eisenhardt and Graebner (2007) note that some areas of research do not have enough existing evidence to build and test hypotheses. While there is a tremendous amount of research in the areas of change, TS, and stakeholder theory, at this point in time, we have limited understanding of how these three areas intersect. Accordingly, the approach taken within this thesis serves to build theory rather than to test theory. 
We limit the scope of this research in two important ways. First, as mentioned earlier, this research considers the views of employees working for each of the three organizations who are part of the TS involved in our case study. We conceptualize each of these organizations as stakeholders and use stakeholder theory to help us understand the impact of planned change on interorganizational relationships. A stakeholder is an individual, group, or organization that may influence or may be influenced by another organization (Frooman, 1984). While it is quite likely that other stakeholders, such as the government or the community, may impact these three organizations and the TS, this research focuses on how the planned TS change impacts only the three organizations involved in the PMI process over time.

Second, this research investigates how the introduction of a planned change impacts relationships within the TS at two points in time (i.e., before a planned change, and six months after the implementation of the change). This research does not, however, directly investigate the myriad of processes (e.g., negotiating, decision-making) occurring at multiple levels of analysis (e.g., individual, organizational, transorganizational) that may also affect the process of TS change. While this research does not neglect the importance of these micro processes, it does not strive to develop theory in any of these areas.

\subsection{RESEARCH CONTRIBUTIONS}

This dissertation contributes to the change, TS, and stakeholder literature in the following ways. First, this research contributes to change theory by developing a framework describing how a planned TS change impacts perceptions of interorganizational relationships over time. The theoretical framework will guide future research on the topic of TS change, and facilitate a comparison of how change takes place in an organization versus how change takes place in a TS. This framework can also help managers who find that their employees are resisting TS changes. 
Second, this research contributes to the TS change literature by exploring how a TS changes without acquiring or removing an organization from the TS. As the majority of the TS change literature focuses on the formation of new and dissolution of existing relationships (Kim et al., 2006), this research contributes to the much smaller body of literature that investigates how interorganizational relationships may change over time (e.g. Gulati, 1998). This case study of changes in interorganizational relationships may also help practitioners understand how changes in their organization impact changes in their partners, and vice versa.

Third, this research also contributes to the stakeholder literature by establishing the linkage between interorganizational relationships and change over time. Given that we ground our understanding of interorganizational relationships in the stakeholder literature, this longitudinal study should offer an important contribution to stakeholder theory, which has largely been viewed from a static perspective (Key, 1999). As this research shows how a planned TS change may impact stakeholders, it may also help practitioners develop planned changes that better manage their relationships with their stakeholders.

\subsection{ORGANIZATION OF THE DISSERTATION}

This dissertation is divided into seven chapters, including this introductory chapter. Chapter 2 reviews the literature used to theoretically ground the research study. This chapter is divided into three main sections: the nature of planned change, transorganizational systems, and stakeholder theory. Chapter 3 presents and justifies the orienting framework for this research. As the framework is based on change, TS, and stakeholder models, this section will also briefly review existing empirical studies in the area and relate it to relevant components of the framework. Chapter 4 features the research design that will be used to conduct this research. This chapter is divided into seven main sections: case study methodology, context, unit of analysis, sampling 
strategy, data collection, interactional experience, and data analysis. The presentation and discussion of findings are shown in Chapter 5 using a change management lens and in Chapter 6 using a stakeholder management lens. Lastly, the concluding chapter addresses the two research objectives, identifies theoretical and practical implications, highlights major contributions, and acknowledges limitations and directions for future research. 


\section{CHAPTER 2: LITERATURE REVIEW}

This literature review has two objectives. First, it seeks to provide the reader with an understanding of the theory and research that frames this dissertation. Second, the review enabled us to identify and construct the conceptual framework guiding this research. Three main bodies of literature were examined as part of this investigation: planned change, TS change, and stakeholder theory.

Section one provides an overview of the literature describing the nature of planned change. Section one is broken down into five subsections. Subsection one situates this study of change within the much broader change management literature. Subsection two defines planned and emergent change. Subsection three reviews Lewin's approach to planned change. Subsection four describes factors affecting planned change. The last subsection critiques the planned change literature and identifies gaps in the literature that will be addressed by this research.

Section two reviews the relevant TS change literature. This section is divided into three subsections. The first subsection overviews four different ways that TSs may change. The second subsection describes factors that may drive or shape TS change. The last subsection, subsection three, critiques the TS literature and identifies gaps to be filled by this research.

Section three presents the relevant stakeholder literature. This section has five parts. In part one, we define the concept of a stakeholder and establish the relevance of this idea to this research. The second subsection outlines the three approaches researchers have taken to study stakeholders. Subsection three reviews stakeholder classifications and then identifies which stakeholder classification is to be used in this research. In subsection four, stakeholder action is described in terms of the selected stakeholder classification. The last subsection critiques the field and identifies gaps in the literature that will be addressed by this research. 


\subsection{THE NATURE OF PLANNED CHANGE}

Change is a complex subject that has been studied from many different perspectives. The purpose of this section of the literature review is to examine relevant theories, concepts, and empirical research that may contribute to an understanding of how planned change unfolds in a TS over time. This section has five subsections. Subsection one summarizes Van de Ven and Poole's (2005) framework conceptualizing approaches to studying change. This framework is then used to situate this dissertation in the existing change management literature. Planned and emergent change are defined in subsection two. Even though this research focuses on a planned change, we expect that some emergent changes may take place as well. In subsection three, Lewin's (1946a; 1946b) approach to planned change is reviewed, and justification for its application in this study presented. In subsection four, the literature about some of the main contextual factors of possible relevance to this thesis are discussed. The last subsection critiques the planned change literature and identifies ways that this research may begin to address these theoretical gaps.

\subsubsection{APPROACHES TO STUDYING CHANGE}

According to Hart (1998), a literature review needs to identify the assumptions that underpin the words under discussion, and evaluate the arguments and findings of particular books and articles of relevance to the research questions of interest. The first section of the literature review does this by clearly articulating some of the assumptions in the organizational change literature that will be adhered to in this study.

A paradigm is a general perspective or way of thinking that reflects fundamental beliefs and assumptions about the nature of organizations (Kuhn, 1974). Researchers do not always explicitly state the paradigms/assumptions underlying their work related to organizational change, even though assumptions are used to anchor theoretical arguments and displays of logic (Yin, 
2003; Yin, 2009). This may, in part, be because the current terminology related to organizational change is imprecise and ambiguous (Marshak, 2002), and thus fails to identify the object of study appropriately (Morgan \& Sturdy, 2000). This section, therefore, clarifies terminology and explicitly states assumptions that will be used throughout this research using Van de Ven and Poole's (2005) framework of approaches to studying change to guide the discussion.

Van de Ven and Poole's (2005) review of the organizational change literature examined how change theorists have studied organizational change and what assumptions they have made. Van de Ven and Poole (2005) grouped their findings based on the epistemology (i.e., the methodological choice) and ontology (i.e., the perspective of organizations) that were used by the researcher to study change.

\subsubsection{EPISTEMOLOGICAL APPROACHES TO STUDYING CHANGE}

There are two definitions of change often used in organization studies: (1) "empirical observation of difference in form, quality, or state over time in an organizational entity. The entity may be an individual's job, a work group, an organizational strategy, a program, a product or the overall organization" (Van de Ven \& Poole, 1995, p. 512), and (2) a narrative describing a sequence of events on how development and change unfold (Poole et al., 2000). When the first definition is used, change is typically studied from a variance perspective (Mohr, 1982), whereas researchers who use the second definition of change typically study change using a processual approach. Table 1 provides a summary comparison of these two approaches, based on the discussion in Poole et al. (2000).

\begin{tabular}{|c|l|}
\hline Variance Approach & \multicolumn{1}{c|}{ Process Approach } \\
\hline Fixed entities with varying attributes & $\begin{array}{l}\text { Entities participate in events and may } \\
\text { change over time }\end{array}$ \\
\hline
\end{tabular}




\begin{tabular}{|l|l|}
$\begin{array}{l}\text { Explanations based on necessary and } \\
\text { sufficient causality }\end{array}$ & $\begin{array}{l}\text { Explanations based on final, formal, and } \\
\text { efficient causality }\end{array}$ \\
\hline $\begin{array}{l}\text { Generality depends on uniformity } \\
\text { across contexts }\end{array}$ & $\begin{array}{l}\text { Generality depends on versatility across } \\
\text { cases }\end{array}$ \\
\hline $\begin{array}{l}\text { Time ordering among independent } \\
\text { variables is immaterial }\end{array}$ & $\begin{array}{l}\text { Time ordering of independent events is } \\
\text { critical }\end{array}$ \\
\hline Emphasis on immediate causation & $\begin{array}{l}\text { Explanations are layered and } \\
\text { incorporate both immediate and distal } \\
\text { causation }\end{array}$ \\
\hline $\begin{array}{l}\text { Attributes have a single meaning over } \\
\text { time }\end{array}$ & $\begin{array}{l}\text { Entities, attributes, events may change } \\
\text { in meaning over time }\end{array}$ \\
\hline
\end{tabular}

Table 1: Comparison of Variance and Process Approaches (Poole et al., 2000)

The variance perspective is employed when researchers view the change being studied as a dependent variable, which may be explained by a set of independent variables (Mohr, 1982; Van de Ven \& Poole, 2005). Studies that take a variance approach employ experimental and survey research designs, grounded in the general linear model (e.g., ANOVA, regression, factor analysis, structural equation modeling). The goal of these studies is to explain and/or predict the occurrence and magnitude of the change, or the effects of change on other variables. Probably the most common type of variance study treats the change construct as a variable, such as rate of innovation (Rogers, 2004) or depth of change (Harrison, 1970). Variance methods have been the dominant approach in studies of organizational change (Poole et al., 2000).

Conversely, process approaches apply to cases where researchers study change by explaining it as a temporal order or sequence in which change events occur (Pettigrew et al., 2001), and are typically based on a story or historical narrative (Van de Ven \& Poole, 2005). Process approaches tend to be more complex than variance approaches because they consider: (1) the 
complexity of events, (2) the temporal connections among events, (3) the different time scales in the same process, and (4) the dynamic nature of processes (Van de Ven \& Poole, 2005). Process theories may incorporate several different types of effects into their explanations including critical events, turning points, contextual influence, formative patterns that give overall direction to the change, and factors that affect the sequencing of events (ibid). As time and time ordering of independent events are critical to the process approach, this type of research requires methods that can identify and test temporal linkages between events and overall temporal patterns (Poole et al., 2000). Most process based research is qualitative in nature and involves a longitudinal research design (Langley \& Abdallah, 2011; Langley, Smallman, Tsoukas, \& Van de Ven, 2013).

\subsubsection{ONTOLOGICAL APPROACHES TO STUDYING CHANGE}

Van de Ven and Poole $(2005, \mathrm{p}$.1387) use the term ontology to refer to whether the researcher perceives the organization as either 'a noun, a social actor, or a real entity ("thing")' or 'a verb, a process of organizing, or emergent flux.' The dichotomy of whether organizations are viewed as consisting of things or processes has been well researched (Tsoukas \& Chia, 2002; Weick \& Quinn, 1999; Van de Ven \& Poole, 2005; Whetten, 2005) and has taken on its own lexicon: strong versus weak forms of organizational change (Tsoukas, 2005), organization versus organizing (Weick, 1979), and things versus processes (Rescher, 1996).

Although expressed in different terms, the distinction between viewing organizations as consisting of things or processes is deeply embedded in the current organizational literature (for review of ontological approaches, see Chia and Langley, 2004 and Whetten, 2005). Within their 'Call for papers' for Organization Studies Summer Workshop, Chia and Langley (2004) distinguish the organization from organizing in terms of strong (i.e. process) and weak (i.e. variance) ways of examining organizational change: 
'The 'weak' view treats processes as important but ultimately reducible to the action of things, while the 'strong' view deems actions and things to be instantiations of processcomplexes. The first perspective appears dominant in much of organizational and social scientific research, and tends to be pragmatic, empirically grounded, and analytical in orientation. The latter perspective has been primarily conceptual, strongly informed by strands of process philosophy, theology and the humanities at large, following especially the lead of philosophers such as James, Whitehead, Bergson, and Deleuze."

Whetten (2005), on the other hand, notes that while valuable insights have been obtained from advocates of the 'strong' view of change, there are four negative implications of treating the 'organizational' in organizational studies as an incidental, inconsequential modifier. Whetten (2005) argues that treating organizations as verbs rather than nouns: (1) threatens the identity of organization studies, (2) jeopardizes the validity of studies in organizations, (3) imperils the coherence of studies of organizations, and (4) fosters overly simplistic explanations and overly general conclusions.

\subsubsection{APPROACHES TO STUDYING CHANGE}

Van de Ven and Poole (2005) use the concepts of epistemology and ontology to conceptualize four possible approaches for studying organizational change (see Figure 1). According to Van de Ven and Poole (2005), assumptions can be made about research that follows each of these approaches to studying change. Approach 1 research assumes the organization is a thing, and change is a dependent variable. Approach 2 research assumes the organization is a thing, and change is a process. Approach 3 research assumes the organization is in emergent flux, and 
change is a process. Approach 4 research assumes the organization is in emergent flux, and change is a dependent variable.

\begin{tabular}{|c|l|l|l|}
\hline & & \multicolumn{2}{|c|}{ An organization is represented as being: } \\
\hline \multirow{2}{*}{$\begin{array}{c}\text { Epistemology } \\
\text { (Method for } \\
\text { studying } \\
\text { change) }\end{array}$} & $\begin{array}{l}\text { Variance } \\
\text { method }\end{array}$ & $\begin{array}{l}\text { A noun, a social actor, } \\
\text { A real entity ("thing") }\end{array}$ & $\begin{array}{l}\text { A verb, a process of organizing, } \\
\text { emergent flux }\end{array}$ \\
\cline { 2 - 5 } & $\begin{array}{l}\text { Process } \\
\text { Variance studies of change in } \\
\text { organizational entities } \\
\text { by causal analysis of independent } \\
\text { variables that explain change in } \\
\text { entity (dependent variable)? }\end{array}$ & $\begin{array}{l}\text { Approach IV } \\
\text { Variance studies of organizing } \\
\text { by dynamic modeling of agent-based } \\
\text { models or chaotic complex adaptive } \\
\text { systems }\end{array}$ \\
\hline & Narratives & $\begin{array}{l}\text { Approach II } \\
\text { Process studies of change in } \\
\text { organizational entities narrating } \\
\text { sequence of events, } \\
\text { stages or cycles of change in the } \\
\text { development of an entity }\end{array}$ & $\begin{array}{l}\text { Approach III } \\
\text { narrating emergent actions and } \\
\text { activities by which collective } \\
\text { endeavors unfold }\end{array}$ \\
\hline
\end{tabular}

Figure 1: Approaches to Studying Change (Van de Ven \& Poole, 2005)

Van de Ven and Poole's (2005) framework can be used to situate this research to both epistemology and ontology. As mentioned in the first chapter, the primary purpose of this thesis is to develop a framework describing how a planned TS change impacts perceptions of interorganizational relationships over time. The fact that we are studying the impact change has on a TS over time means that we are viewing the phenomena through a processual lens (Langley, Smallman, Tsoukas, \& Van de Ven, 2013). This research also conceptualizes the TS, as well as the organizations that make up the TS, as entities (i.e. real things). These two assumptions (i.e., change is a process, TS is an entity) mean that this research follows an Approach 2 methodology to studying change (Van de Ven \& Poole, 2005).

\subsubsection{METHODOLOGICAL IMPLICATIONS}


Studying TS change from an Approach 2 perspective has numerous methodological implications. First, Approach 2 research views the flow of time as irreversible and the temporal succession of events as a developmental process (Van de Ven \& Poole, 2005). The fact that this approach involves the study of events over time means longitudinal data collection is required. More detail on the longitudinal data collection for this research is provided in Chapter 4.

Second, Approach 2 studies focus on the temporal occurrence of significant events. This view regards time as "divisible, but differentiated, with certain points serving as 'critical values" (McGrath \& Kelly, 1996, p.33). Van de Ven and Poole (2005) argue that data should be collected before and after critical events (e.g., before and after a change) to capture the rich data that describes the processes. Thus, the identification of these 'critical' points in time are vital to Approach 2 studies. As described in more detail in section 4.5, this research collected data at two critical points in time: before changes were implemented and 6 months after changes were implemented.

\subsubsection{PLANNED AND EMERGENT CHANGE}

Researchers who are interested in how change comes about focus their discussions on two types of change: planned and emergent (Bamford \& Forrester, 2003). Planned change refers to situations where management deliberately make decisions to change something and where it is assumed that the results of the change can be anticipated (Todnem By, 2005). This may be contrasted with emergent change, where outcomes of change are unexpected, and the consequences of change are unanticipated (ibid). While this thesis focuses on a planned change within a TS, we also expect that emergent changes could take place (Van de Ven \& Poole, 2005). The first part of this section (Section 2.1.2.1) describes research on planned change, while the second part (Section 2.1.2.2) describes research on emergent change. 


\subsubsection{PLANNED CHANGE}

Planned organizational change, as defined by Porras and Silvers (1991, p.52), is:

"a change intervention that alters key organizational target variables that then impact individual organizational members and their on-the-job behaviors resulting in changes in organizational outcomes."

Most planned organizational change models implicitly assume that an organization exists in different states at different times, and that planned change can move the organization (or parts of it) from one state to the next (Burnes, 1996). These assumptions are consistent with the selection of an approach 2 perspective of change in this research (Van de Ven \& Poole, 2005). In most cases of planned change, managers initiate and implement changes, moving the organization from the current state to the desired state, to respond to perceived opportunities to improve organizational performance or achieve a better fit with the environment (Porras \& Silvers, 1991; Orlikowski, 1996). The outcomes of such change initiatives are presumed to be knowable in advance and also, somewhat, controllable (Olikowski, 1996). Most planned change models can be placed in the category of prescriptive literature, as they are dealing with the question of how an organization should change. Planned change models have traditionally dominated the organizational change literature (Orlikowski, 1996).

Planned change research assumes managers play a critical role in organizational change (Tsoukas \& Chia, 2002) and articulates problems of the organization solely from the standpoint of managers (Knights \& McCabe, 2002), particularly senior managers (Balogun, 2006). Planned change models are popular with managers because they legitimize the role of management in the proposed solution (Clark \& Salaman, 1998). The also allow the identity of the manager to be constituted as that of a heroic, transformational leader (ibid). 
The emphasis on the role of managers in change initiatives seems somewhat limited, as scholars have argued that frontline employees are the ones who are changing the organization (Pfeffer \& Veiga, 1999). Few studies have been conducted that explore perceptions of frontline employees throughout the process of planned change. Research by scholars such as Jaffe et al. (1994) and Reay, Golden-Biddle, and Germann (2006) show that inclusion of the perspective of frontline employees in studies of planned change can contribute to our understanding of how planned TS change may impact perceptions of interorganizational relationships over time. Accordingly, this approach to studying planned change (i.e. collecting data from frontline workers) is used in this dissertation (see data collection in Section 4.5).

\subsubsection{EMERGENT CHANGE}

Emergent change is involuntary (Mangham, 1980) and unintended (Armenakis \& Bedeian, 1999) change. Orlikowski (1996, p.65) describes it as "the realization of a new pattern of organizing in the absence of explicit, a priori intentions". That is, the outcome of a change initiative cannot be foreseen at the beginning of the initiative, as change is dynamic and interdependent on many other factors that may also be evolving throughout the change (Van de Ven \& Poole, 2005). The very nature of emergent change means that it only becomes evident retrospectively (Chia, 1999).

The emergent change literature does not deny that planned change may lead to intended outcomes (Balogun \& Johnson, 2005). This literature does argue, however, that change initiatives are also likely to result in unintended outcomes given the complexity of the change process (Weick \& Quinn, 1999). The literature that views change as an emergent phenomenon suggests the change is so rapid that it is impossible for senior managers effectively to identify, plan, and implement the necessary organizational responses (Kanter et al., 1992). This may be why, in contrast to the 
planned approach that tends to see change as top-down driven, the emergent approach tends to see change driven from the bottom-up (Bamford \& Forrester, 2003). This approach emphasizes that change should not be perceived as a series of linear events within a given period of time, but as a continuous, open-ended process of adaptation to changing circumstances and conditions (Burnes, 1996; 2004).

Burnes (1996) argues the general applicability and validity of the emergent approach to organizational change depend on whether or not one believes that all organizations operate in dynamic and unpredictable environments to which they constantly have to adapt. If so, Burnes (1996, p.14) argues "the emergent model is suitable for all organizations, all situations, and at all times.” Burnes' (1996) logic would suggest that the emergent change approach is consistent with research based on Van de Ven and Poole's (2005) Approach 3 or Approach 4, which stress 'processes of organizing' and 'emergent flux.' As this dissertation's assumptions are consistent with Van de Ven and Poole's (2005) Approach 2, which stress an organization is an entity, this research is more consistent with literature that uses the planned change approach, however, acknowledges those researchers (e.g. Armenakis \& Bedeian, 1999) who argue that the implementation of planned change may result in intended and unintended outcomes.

The next section discusses the most prominent model of planned change in the organizational literature: Lewin's (1946a; 1946b) approach to planned change (Burnes, 2004). Lewin's model of planned change involves multiple stages and its use in this study is consistent with Van de Ven and Poole's (2005) approach 2 to studying change.

\subsubsection{LEWIN'S APPROACH TO PLANNED CHANGE}

According to Burnes (2004), Lewin's approach to planned change includes: (1) field theory, (2) group dynamics, and (3) the 3-step model. While these three theories are imperative to 
the study of change (Burnes, 2004), we also believe that Lewin's gatekeeping theory is critical to understanding how change unfolds in a TS over time. The subsections below explain Lewin's four major theoretical contributions: field theory, group dynamics, gatekeeping theory, and the 3-step model.

\subsubsection{FIELD THEORY}

Field theory is an approach to understanding group behavior by trying to map out the totality and complexity of the field in which the behavior takes place (Back, 1992; Burnes, 2004). Lewin argued that understanding the present situation, or the status quo as he terms it, requires the understanding of "certain conditions and forces" (Lewin, 1943, p. 172). Lewin (1943) hypothesized that synthesizing active forces at any given time produces the group environment, or in Lewin's words: the field. Lewin defined a field as "a totality of coexisting facts which are conceived of as mutually interdependent..." (Lewin, 1946a, p. 240) and postulated that individual behavior is a function of the field (1947b). This research applies Lewin's concept of a field to a TS (i.e. PMI process) consisting of multiple mutually interdependent organizations.

Lewin's view was that if one could identify, plot, and establish the magnitude of these forces, then it would be possible not only to understand why individuals, groups, and organizations behave as they do, but also what forces would be required to bring about change. Lewin (1947a) believed that a field was in a continuous state of adaptation and that "change and constancy are relative concepts; group life is never without change, merely differences in the amount and type of change exist" (p. 199). Despite the flow of a field, Lewin saw a behavioral change as a slow process structured around a "quasi-stationary equilibrium" where the effects of forces were offset by that of their counterparts. However, Lewin did recognize that under certain circumstances, such as personal, social, or organizational crisis, the various forces in the field could shift quickly 
and radically. In such situations, established routines and behaviors break down and the status quo is no longer viable; new patterns of activity can rapidly emerge, and a new equilibrium is formed (Kippenberger, 1998; Lewin, 1947a).

More recent research on field theory of change has focused on identification and summation of forces impacting such change. Researchers working in this area have identified different types of forces that may impact change, such as funding, legislation, innovation and competitors (Battilana \& Casciaro, 2012; Elie-Dit-Cosaque, Pallud \& Kalika, 2011; Jones, 2010; Adler \& Koehn, 2012; Lunenburg, 2010). Typically, researchers conduct an analysis of some or all of these forces to provide information that will allow them to manage change better (Schein, 1996). The tool used to conduct such an analysis is called a force field diagram. A force field analysis is a framework for understanding how forces influence a situation and identifying which resultant forces need to be strengthened or diminished to bring about change (Lewin, 1946b).

Lewin argued that the equilibrium (i.e., current state) needs to be destabilized before old behavior can be discarded and new behavior successfully adopted. That is to say that the driving forces of change need to be greater than the restraining forces of change. Thus, the identification of change drivers and change restrainers may offer managers a clearer picture of what needs to be levered to bring about change.

Driving forces of change are "events, activities or behaviors that facilitate the implementation of change" (Whelan-Berry, Gordon \& Hinings, 2003, p. 100). These forces have been given multiple names over the years (e.g., change drivers, change accelerator, catalysts, action levers; Jick, 1995; Whelan, 1997; Porras \& Hoffer, 1996), although the underlying concept of increasing the strength of force to unfreeze a force field remains the same. Forces for change can be internal or external. Some examples of internal driving forces of change include vision, 
leadership, human resources, communication and enabling changes in structure and processes (Whelan-Berry, Gordon \& Hinings, 2003; Bridges, 2003; Nadler \& Tushman, 1990; Kemelgor, Johnson \& Srinivasan, 2000). Some examples of external driving forces of change include stakeholders or government (e.g., Den Hond \& de Bakker, 2007; Government of Ontario, 2012). Studies of successful change initiatives revealed that the adoption of multiple change drivers involving adjustments of structural arrangements and organizational processes is often necessary (Pettigrew et al., 2001).

One driving force for change that has received increasing attention in the literature is readiness to change (Jones, Jimmieson \& Griffiths, 2005). Readiness to change is reflected in organizational members' beliefs, attitudes, and intentions regarding the extent to which (1) the changes are needed and (2) the organization's ability to change (Armenakis, Harris \& Mossholder, 1993). Because the energy, inspiration, and support necessary to create readiness must come from within an organization, readiness is most often associated with internal change drivers (i.e., organizational leaders, managers; Armenakis et al., 1993).

Restraining forces of change, which have also been referred to as barriers to change and forces impeding change (e.g., Argyris, 1993; Post \& Altma, 1994; Burke, 2010), are defined as events, activities or behaviors that promote the status quo (Lewin, 1946a). Restraining forces can be internal or external. Several examples of internal restraining forces that have been identified in the literature are communication problems, group norms, organizational culture, senior management and lack of planning (e.g., Burke, 2010; Post \& Altma, 1994). Several examples of external restraining forces that have been shown in the literature are legal constraints and stakeholders (e.g., Burke, 2010; Post \& Altma, 1994). 
Resistance to change has been identified as a major barrier to change. Resistance to change was originally identified by Coch and French (1948) and later studied by a series of researchers (Dent \& Goldberg, 1999; Piderit, 2000; Kotter \& Schlesinger, 1979). Resistance to change has been historically defined as "behavior which is intended to protect an individual from the effects of real or imagined change" (Zander, 1950, p.9). Researchers have identified different forms of resistance. For example, Morrison and Milliken (2000) theorize that lower level employees may resist change by staying silent and not suggesting things the manager may disagree with for fear that the manager will punish them. Or, del Val and Fuentes (2003), in their review of resistance to change, identified five different sources of resistance to change: (1) perceptions of need to change, (2) low motivation for change, (3) lack of creative response, (4) political, and (5) cultural deadlocks and different characteristics. More recently, Ford, Ford, and D'Amelio (2008) argue that resistance can be more appropriately understood as a dynamic among three elements: (1) recipient action, which is any behavior or communication that occurs in response to a change initiative; (2) agent sensemaking, including agents' interpretations of and meanings given to actual or anticipated recipient actions as well as the accounts agents take as a function of their own interpretations and meanings; and (3) agent-recipient relationship, which provides the context in which the first two elements occur. Whatever form resistance to change takes, it has been traditionally viewed as a force from employees resisting change and thus can be costly for managers trying to change their organizations (Ford, Ford \& D'Amelio, 2008).

There have been many strategies developed to overcome resistance to change (see reviews by Kotter \& Schlesinger, 1979; Dent \& Goldberg, 1999; Morrison \& Milliken, 2000; Ashforth \& Mael, 1998). For example, Armenakis and colleagues (1993) suggest that managers should take a more proactive role in organizational change by readying their employees mentally and physically 
for the proposed change. Kotter (1995) argues that leaders who develop a sense of urgency (i.e. reason for the change) are more likely to overcome initial resistance than those who do not. Kan and Parry (2004) find that organizational change breeds paradoxes and paradoxes breed resistance to change as individuals do not understand their new role in the organization. Kan and Parry (2004) argue that the development of paradoxes and resistance to change can be overcome by identifying and reconciling the perceived paradoxes.

\subsubsection{GROUP DYNAMICS}

Lewin (1947a, p. 165) defines a group by stating: “... it is not the similarity or dissimilarity of individuals that constitutes a group, but interdependence of fate". Even though Lewin (1947a) speaks about a group in terms of individuals that are interdependently related, his theory can be applied to this research, which involves a group of organizations that are interdependently related by virtue of their involvement in the PMI process.

Group dynamics stresses that group behavior, rather than individual behavior, should be the main focus of a change (Dent \& Goldberg, 1999). Lewin (1947a) argues that it is fruitless to concentrate on changing individual behavior because the individual in isolation is constrained by group pressures to conform. In the context of TS change, therefore, this would imply: (1) change takes place within the three organizations that are part of the TS, and (2) change efforts should focus on group level factors such as group norms, roles, interactions, and socialization processes to create disequilibrium and change (Schein, 2002). In other words, the behavior of the individual organization within the TS is constrained by TS pressures, and thus less likely to affect how planned change unfolds in a TS over time.

Lewin's (1947a) group dynamics theory has been extended by social psychologists, such as Strasser and Titus (1985), Tindale and Kameda (2000), and Kameda et al. (2002), who 
emphasize the importance of 'sharedness' between group members. 'sharedness' is based on two ideas: (1) groups of individuals can be conceptualized as information-processing systems, and (2) the degree to which cognitions, preferences, etc. are shared and are being shared within the group affects group dynamics (Tindale \& Kameda, 2000). The idea that things that are shared among group members tend to have an inordinate impact on group decisions and behaviors (Kameda et al., 2002) is consistent with Lewin's (1946a) theory of group dynamics, which suggests that group pressures constrain individuals.

The study of 'sharedness' has been gaining considerable attention in the organizational literature. For example, the notion of 'sharedness' has been applied to teams (e.g., Huber \& Lewis, 2010; Rico, Sanchez-Manzanares, Gil, \& Gibson, 2008; Cronin \& Weingart, 2007; Grutterink, Van Der, Molleman, \& Jehn, 2010; Gibson \& Earley, 2007; Celani \& Tasa, 2010). It is also related to the concept of sensemaking (Weick, 1995; Weick et al., 2005) in that sensemaking is (1) grounded in identity construction (i.e. meaning individuals' mental schema will influence how they interpret events), (2) enactive (i.e. meaning that it is a process that allows individuals to translate meaning into action), and (3) social (i.e. meaning individuals consult others and talking is central) (see Weick's (1995) properties of sensemaking). As sensegiving reflects one individual contributing to another's ability to make sense of an event, 'sharedness' may also be linked to sensegiving (e.g., Balogun, 2003; Maitlis \& Lawrence, 2007). More specifically, sensegiving may contribute to the extent that perceptions are shared between individuals.

Unfortunately, the majority of research on group dynamics and 'sharedness' has approached these phenomena from the context of a single organization. This dissertation research extends this literature by exploring these phenomena within three organization implementing a planned TS change. 


\subsubsection{CHANNELS AND GATEKEEPERS THEORY}

Even though Lewin (1947b) argues that change initiatives should be directed at the group level, he also notes that certain individuals in the group are uniquely positioned to facilitate change. It was for this reason that he developed the theory of channels and gatekeepers.

Lewin's $(1947 \mathrm{a} ; 1947 \mathrm{c})$ theory of channels and gatekeepers explores how certain individuals within a group may influence the process of change more than others within the group. In conceptualizing this theory, Lewin (1947a) assumes that not all members of a group are equally important in determining how change unfolds within the group over time. In so doing, Lewin (1947a) contends that social change can best be understood by concentrating on those people with the most power within the group, whom he refers to as "gatekeepers." According to Lewin (1947a), gatekeepers are individuals within a group who make critical group decisions and, as a result, may directly impact the group over time.

In his original work, Lewin (1947a) provides the example of food preparation when describing his theory of channels and gatekeepers. Lewin (1947a) discusses the process of food preparation consisting of a series of steps, which he refers to as channels. One channel could be the grocery store, where food is selected and purchased, while another channel may be the kitchen, where food is cleaned and cooked. It should be noted that potential channels may overlap, such as in the case of how an individual obtains the food (e.g., from a family garden, from the grocery story, from a neighbour). In each of these channels, there are gatekeepers who control the movement of the food within the channel. For example, in the grocery store channel, a shopper (i.e. the gatekeeper of the grocery store channel) may decide to buy corn instead of potatoes because it was on sale. In the kitchen channel, the cook (i.e. the gatekeeper) may decide to boil the corn instead of cooking it on the barbecue. These gatekeepers make decisions within their 
respective channel (i.e., what to buy, how to cook it) that influence how the food will be prepared and, thus, may decide to change how the food was previously prepared. Thus, according to Lewin's (1947a) reasoning, how the process of change unfolds may best be understood by concentrating on those gatekeepers who are making key decisions that can affect changes in the system over time.

Lewin (1951, p.187) believed that his theory of channels and gatekeepers could generally be applied:

"This situation holds not only for food channels but also for the traveling of a news item through certain communication channels in a group, for movement of goods, and the social locomotion of individuals in many organizations. A university, for instance, might be quite strict in its admission policy and might set up strong forces against the passing of weak candidates. Once a student is admitted, however, the university frequently tries to do everything in its power to help everyone along. "

In the context of this research on TS change, Lewin's (1947a) channel and gatekeeper theory may apply to how the three organizations within the TS behave within the PMI process. One may argue that the PMI process could be broken down into different channels including apprehension and assessment. In such a conceptualization, the apprehension channel may include activities related to the process of police officers arriving on the scene, talking with witnesses, and deciding whether the PMI should be apprehended; while the assessment channel may include activities related to the process of healthcare professionals triaging and assessing the PMI physically and mentally. This conceptualization would imply that: (1) police officers may be gatekeepers within the apprehension channel, and (2) healthcare professionals may be gatekeepers within the assessment channel. As employees of the police and hospital may be gatekeepers within 
the PMI process, they may also be uniquely positioned to affect how planned TS change impacts TS stakeholder relationships over time.

While Lewin's research on group dynamics and gatekeeping theory contribute to our understanding of the group's and individual's role in the process of change, understanding the internal dynamics of a group is not sufficient by itself to bring about and sustain change. Lewin (1947a, p. 228) was concerned that

"after a 'shot in the arm', group life soon returns to the previous level... This indicates that it does not suffice to define the objective of a planned change in group performance as the reaching of a different level. Permanency at the new level... should be included in the objective."

As suggested by the above quote, Lewin (1947a) distinguishes between creating and sustaining change. As conceptualized in his 3-Step Model of change, Lewin (1947a) believes that change initiatives should be designed to both establish and sustain change.

\subsubsection{LEWIN'S 3-STEP MODEL}

The conceptualization of change as a 3-step procedure is probably what Lewin is best known for among change management researchers (Burnes, 2004). Lewin (1947a) postulated that change unfolds in a system (e.g., organization, TS) through three successive levels of change: (1) unfreezing the present level, (2) moving to the new level, and (3) refreezing the new level (see Figure 2). Each level, or step, corresponds to a particular equilibrium, which is determined by the forces maintaining the quasi-stationary equilibrium at each point in time (Lewin, 1947a).

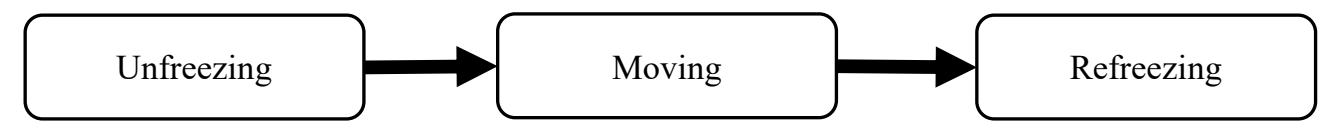

Figure 2: Lewin's 3 Step Model 
Unfreezing refers to the processes involved in destabilizing a force field (Lewin, 1947a) and allows individuals (or organizations) to unlearn old behaviors and learn new behaviors (Burnes, 2004). As mentioned previously, force field destabilization occurs when driving forces of change are greater than restraining forces of change.

Once the system is unfrozen, it can move to a new state and take on new behaviors (Lewin, 1947a). The moving stage of Lewin's 3-Step Model of change is theorized as the process of trialand-error through implementation and alteration of change efforts (Lewin, 1947b), and is consistent with Schein (1996, p.62) who notes that unfreezing is not an end in itself; it “...creates motivation to learn but does not necessarily control or predict the direction."

To reinforce changes made during the moving step, refreezing needs to occur (Lewin, 1947a). Refreezing refers to the stabilization of change at a new quasi-stationary equilibrium to ensure that new behaviors are relatively safe from regression (Cummings \& Worley, 2009). An important aspect about refreezing is that new behavior must be, to some degree, congruent with the rest of the behavior, personality and environment of the learner or it will simply lead to a new round of disconfirmation (Schein, 1996). This is why Lewin saw the permanency of change as a group activity, because unless the group is also transformed, changes to individual behavior will not be sustained: "If one succeeds in changing group standards, this same force field will tend to facilitate changing the individual and will tend to stabilize the individual conduct on the new group level" (Lewin, 1947a).

\subsubsection{FACTORS AFFECTING PLANNED CHANGE}

Lewin's approach to planned change reviewed in the previous section describes how change is conceptualized to unfold in organizations (or in this case in TSs) over time. In reality, the change process is not nearly as orderly as the model implies. The steps of planned change can 
shift in a variety of ways depending on factors such as the readiness of the organization to change (Armenakis et al., 1993), the change agent's skills and values (Kotter, 2008), and the organization's context (Pettigrew et al., 2001). Thus, the way change unfolds, even when planned, can vary enormously from one situation to another. Within this section, four contextual factors that are relevant to this dissertation study on planned change within a TS are identified: (1) the magnitude of organizational change (i.e. fine-tuned vs. radical), (2) the rate of change (i.e. episodic vs. continuous), (3) the degree to which the system is organized (i.e. under-organized vs. overorganized), and (4) the sector in which the change takes place (i.e. public vs. private). This section reviews the literature describing each of the above dimensions of change, shows how this factor is conceptualized in the dissertation case study, and outlines how this factor may influence how the planned change being studied unfolds over time (see Table 2 for a summary of this discussion).

\begin{tabular}{|l|c|}
\hline \multicolumn{1}{|c|}{ Factor } & Dissertation Case Study \\
\hline $\begin{array}{l}\text { Magnitude of Change: Fine- } \\
\text { tuning or Radical }\end{array}$ & Radical Change \\
\hline $\begin{array}{l}\text { Tempo of Change: Episodic } \\
\text { or Continuous }\end{array}$ & Episodic Change \\
\hline $\begin{array}{l}\text { Degree of Organization: } \\
\text { Under or Over }\end{array}$ & Under-organization \\
\hline Sector: Public or Private & \multicolumn{1}{|c|}{ Public } \\
\hline
\end{tabular}

Table 2: Summary of Factors Affecting Planned Change

\subsubsection{MAGNITUDE OF CHANGE}

Planned changes within organizations can be characterized as falling along a continuum ranging from fine-tuning exercises to radical, large-scale change efforts. Fine-tuning is also known as convergent change (Greenwood \& Hinings, 1996) and tends to involve limited dimensions and levels of the organization, such as attempting to change a single process within an organization. These planned changes occur within the context of the organization's existing business strategy, 
structure, and culture and are aimed at improving the status quo (Nadler \& Tushman, 1989). Radical change, on the other hand, is often referred to as frame-breaking change (Nadler \& Tushman, 1989; Greenwood \& Hinings, 1996) and is directed at significantly altering how organizations operate. Radical planned changes tend to involve simultaneous changes to several organizational dimensions, including structure, culture, reward systems, information processes, and work design (Nadler \& Tushman, 1989).

Radical changes, sometimes referred to as re-creation, involve a significant break or departure from an organization's past (Nadler \& Tushman, 1989). Because boundaries of radical changes are not absolute or "tangible", as compared to fine-tuning changes, such change typically requires more effort than fine-tuning (Nadler \& Tushman, 1989). Examples of radical changes include changes to the management support structure (Engestrom, Kerosuo \& Kajamaa, 2007), significant changes to revenue streams (Reay \& Hinings, 2005), and the development of new departments (Sillince, Harindranath \& Harvey, 2001).

The dissertation case study involves changes to the PMI process, which might reflect finetuning change at the organizational level (e.g. continuous improvement of the status quo). It is important to note, however, that the main change we are studying takes place at the level of the TS, as organizations will be required to change how they operate within the TS by building structures and developing relationships to facilitate operations between organizations. Accordingly, we feel this case study is likely to involve a radical change at the TS level.

\subsubsection{TEMPO OF CHANGE}

Planned change initiatives can also vary on the tempo of change. The tempo of a change can be understood as the characteristic rate, rhythm, or pattern of work or activity (Weick \& Quinn, 1999). Two tempos of change are commonly discussed in the change literature: episodic and 
continuous. Episodic change refers to "an occasional interruption or divergence from equilibrium" (Weick \& Quinn, 1999, p.366), whereas continuous change involves continually updating of work processes and social practices (ibid). In their comparison of episodic and continuous change, Weick and Quinn (1999) make the case that episodic change is infrequent, discontinuous, and involves substantial divergence from the status quo (see Table 2), while continuous change is constant, evolving, and requires endless modifications. Examples of episodic changes include Apple Computer's discontinuous changes in strategy, structure, and culture as it moved from the leadership of Steve Jobs through that of John Sculley, Michael Sprindler, Gil Amelio, and back to Jobs (Tushman, Rielly \& Charles, 1996). Examples of continuous change, in contrast, include improvisations to standard procedures that lead to improved performance (Moorman \& Miner, 1998; Orlikowski, 1996; Brown \& Eisenhardt, 1998).

It is important to note that there can be an element of planning in both continuous and episodic change. For example, Tsoukas and Chia (2002) argue human agency is always involved in change, whereas Chae and Hill (2000) note that different changes may require various levels of planning for change agents to believe that they can anticipate the outcome of their actions. Interestingly, although Weick and Quinn (1999) equate continuous change to unplanned change, they also note that an intervention may be used to orient continuous change, which would suggest some planning is required to orchestrate the intervention.

As this case study involved a planned change that is discontinuous and radical within the TS, we conceptualize it using episodic change theory. Some theoretical examples of episodic change include punctuated equilibrium (Gersick, 1991), revolutionary (Romanelli \& Tushman, 1994) and replacement (Ford \& Ford, 1994) models, all of which are consistent with Van de Ven and Poole's Approach 2 to studying change. 


\subsubsection{DEGREE OF ORGANIZATION}

Planned change initiatives can also vary depending on the degree to which organizations (or in this case a TS) are organized. Degree of organization refers to the tightness of the structure governing collaborative parties (Roberts, 2004). Over-organization can be found in organizations with numerous organizational dimensions (e.g., policies and procedures) that are too rigid and overly defined for effective task performance (Anderson \& Jap, 2012). Such organizations tend to be described as overly mechanistic and bureaucratic. Under-organization is reflected in collaborations that do not have enough structure for effective task performance (Brown, 1980), such as interorganizational collaborations (e.g., Westley \& Vredenburg, 1991), information technology supply networks (e.g., Gunter, Grote \& Thees, 2006), and community development (e.g., Jamal \& Getz, 1995). In such cases, relationships among diverse groups and participants typically must be organized with complex, uncertain tasks. As the dissertation case study involves changes within a TS, where three diverse groups must collaborate to accomplish a complex and largely unpredictable task, we feel the case typifies an under-organized system.

Planned change in under-organized situations may increase organization by many different strategies including clarifying roles, structuring communication between manager and employee, and specifying responsibilities (Cummings \& Worley, 2009). According to Brown (1980), planned change in under-organized systems require a modification from the steps described in Lewin's 3-Step Model of change and include the following four steps:

1) Identification: This step identifies key individuals and organizations who need to be involved in the planned change. In many under-organized situations, people can be so disconnected that there is ambiguity about who should be included in the planned change (Roberts, 2004). Without the proper identification of who should be involved in 
implementing changes, situations with broad disagreement on what the problem is often result in failed action (Weber \& Khademian, 2008).

2) Convention: In this step, the key individuals and organizational members are brought together to begin organizing efforts for the planned change.

3) Organization: This step involves the creation of different organizing mechanisms to structure the newly required interactions among people and organizations. This may include, for example, the creation of a team that establishes communication channels and coordinates appropriate plans and procedures (Keen, 2003) and then passes that information to the remaining parties.

4) Evaluation: In the final step, the outcomes of the organization stage are assessed and might signal the need for further refinement of the identification, convention, or organization steps.

\subsubsection{CHANGE IN PUBLIC SECTOR}

In addition to the above-reviewed factors, planned change may also be influenced by whether the change takes place in a private or public sector setting (e.g., Pollitt and Bouckaert, 2003; Caldwell, 2009). As the dissertation case study involves three organizations that have been identified as public sector entities within Kuiper et al.'s (2014) recent review of change in public sector organizations, we feel that the dissertation case study involves planned public sector change. Our consideration of the literature identified three differences between the public and private sector, which may impact planned change: bureaucracy, drivers of change, and interorganizational relationships. Details on each of these factors are discussed in the section below, as they may have an impact on how the planned TS change is perceived and unfolds. 
Bureaucracy. One of the fundamental differences between private and public sector firms is the level of bureaucracy (i.e., legislation and rules) within the organization (Kuipers et al., 2014). According to Rainey (1997), bureaucracy relates to organizational processes such as the clarity of organizational goals and the amount of 'red tape'. Healthcare, police, social service agencies, as well as other public sector organizations tend to be impacted by bureaucracy more than that of private sector firms (Boyne, 2002; Wilson, 2000). For example, Mattox (2001) uses evidence from the World Trade Center attack to argue that many of the agencies and departments providing healthcare to victims were unable to act as bureaucracy overwhelmed much of their disaster plans. Moreover, Clark (2005) argues that the traditional bureaucratic models of policing may no longer be functional for policing post-modern society where decentralized decision-making is more effective. Give that this research involves changes to a TS consisting of three public sector organizations, bureaucracy is likely to play a role in how planned change unfolds in the TS over time.

Drivers of Change. As noted previously, drivers of change are forces acting on public sector organizations that challenge the status quo. Major drivers of change are likely to influence the direction of the planned change (Pettigrew et al., 2001). There have been a variety of drivers of change identified in the public sector literature, such as changing client demands (Christiansen, 2006), the availability of new technologies (Vann, 2004), and financial crises (Hendriks \& Tops, 2003). Central government decisions including policy changes and new regulations are typical drivers of reorganization and the implementation of change in healthcare (e.g., Jespersen et al., 2002), policing (e.g., Bayley, 2008) and social service agencies (e.g., Reichard, 2003).

The major forces driving the planned change to the PMI process, which is the focus of this dissertation, were the closure of mental health hospitals (i.e. change in policy) and the increased 
number of non-institutionalized individuals identified with mental illness (i.e. change in public demands). These two forces are consistent with the literature on change drivers in the public sector (Christiansen, 2006; Jespersen et al., 2002).

Intergovernmental Relationships. Governments are designed to disperse power and function so that no one government sector or agency is all-powerful (Kuipers et al., 2014). As a result, government entities are often comprised of networks of independent agencies providing different public services to the same customers (e.g. the PMI process). The result is an intergovernmental relations environment where multiple levels of governments share power, responsibility and, in some cases, resources (Bryson, 2011). These governmental relationships can influence planned change efforts particularly if one key government agency, who holds a required resource, resists the change (Painter, 1991) or is unwilling or unavailable to participate in the planned change. For example, Steadman, Deane, Borum and Morrissey (2000) compared the outcomes of major models of police responses to mental health emergencies (i.e., PMI process) in three American cities. Their data strongly suggest that collaborations between the criminal justice, the mental health system, and the advocacy community plus essential services greatly improved the effectiveness of the PMI process (Steadman et al., 2000). Research by Steadman and colleagues (2000) supports the idea that intergovernmental relationships are of particular importance to changes in a TS consisting of public sector organizations.

This research involves three public sector organizations who each have command over a unique set of resources (i.e., they each have their own expertise and skills), each of which is required to complete the PMI process. If one of these partners resists a particular change to the PMI process, it is likely that TS change would unfold differently than if all partners were 
"supportive" of a particular change. Using Lewin's terminology, the partner in possession of TS resources that are critical to the change is a gatekeeper to the TS change.

\subsubsection{CRITIQUE OF PLANNED ORGANIZATIONAL CHANGE LITERATURE}

Despite the continued refinement of theory describing planned change, our review of the literature identifies a number of areas where the theory is lacking. Below are six critiques of the planned change research and ways that this study intends to address these gaps.

First, Lewin's planned approach to change has been commonly criticized as being too simplistic and mechanistic for a world where organizational change is a continuous and openended process (Burnes, 2004). Those more familiar with Lewin's approach argue however that this is not a valid criticism but instead a blatant misinterpretation of the approach to planned change proposed by Lewin (Burnes, 2004). It was Lewin's intention that field theory, group dynamics, gatekeeping theory, and the 3-Step Model be considered in tandem, not separately. If one considers field theory, group dynamics, gatekeeping theory, and the 3-Step Model as complementary parts of a planned approach to change, it becomes clear that Lewin's approach is not over simplified (Burnes, 2004). It is an approach that nestles group behavior into a complex field of forces. It highlights the dual importance of individual actors (or gatekeepers as Lewin refers to them) and the social context of change. This dissertation echoes Burnes' (2004) response

to this criticism by theoretically grounding this research in three of Lewin's theories (i.e., field theory, group dynamics, and gatekeeping theory). In other words, this research explores employees' perceptions of a planned change to get a better understanding of how they may drive, restrain, and shape interorganizational relationships over time.

A second deficiency with the general conceptualization of planned change in the extant literature involves a lack of knowledge about how the stages of planned change differ across 
situations. Lewin's planned approach to change specifies a general set of steps that are intended to apply to most change efforts. However, as shown in section 2.1.4, change efforts can vary considerably depending on such factors as the magnitude of change, the rate of change, the degree of organization, and 'sharedness'. Accordingly, this research takes the following steps to ensure a rigorous investigation of planned change. We begin by classifying the type of change along five dimensions that the literature of change indicated may have relevance to this study (see Table 2). Doing so allows us to tap into the appropriate research when interpreting our results.

Third, our review of the planned change literature found that the majority of change research uses a variance approach to study change (Van de Ven \& Poole, 2005). This research contributes to the change literature by using a process approach to study TS change.

The fourth critique of planned change research is related to the lack of research on this topic in the public sector literature. Kuipers et al. (2014) concluded his recent review of change in the public sector by noting that there is a need for more "in-depth empirical studies of the change process within various public contexts" (p.16). This study addresses this void in the public sector literature by examining changes in a TS consisting of a hospital, a police organization, and a social service agency.

Within the same review, Kuipers and colleagues (2014) identified a second interesting gap in our understanding of planned change by noting that virtually all studies of change have used two theoretical frameworks: (1) institutional theory, which is context-aware, and (2) generic change theory, which is detail-oriented to processes and behaviors. They argue that scholars need to “... look for opportunities to use the strengths of different theoretical approaches to study the field" (ibid, p.16). This study addresses this gap by using stakeholder theory (see Section 2.3) as 
a form of contextual framework to investigate how the relationships between public sector organizations within a TS may change over time.

Lastly, our review of the planned change literature found that the majority of change research has taken place within single organizations (Burnes \& Cooke, 2012), and as a result, does not fully explain how planned change unfolds in TSs. This research addresses this critique by exploring how planned change unfolds in a TS. The next section reviews the TS change literature.

\subsection{TRANSORGANIZATIONAL SYSTEM (TS) CHANGE}

The literature reviewed in the previous section, which describes how planned change may unfold, is primarily based on research conducted at either the individual or the organizational level. Change in organizations can, however, be considerably different from change in TSs (Crosby, Bryson, \& Stone, 2006; Huxham \& Vangen, 2000). As such, the purpose of this section is to review the growing, yet still small, literature on TS change.

Although an extensive body of knowledge exists on TS outcomes and on how TS structures may contribute to the creation of outcomes (e.g., Powell et al., 2005; Oliver \& Ebers, 1998; Galaskiewicz, 1985), less attention has been paid to understanding how TSs emerge, evolve, and change over time (Ahuja et al., 2012). Thus, this section draws on numerous literatures including strategic alliances (e.g. Das \& Teng, 2000), interorganizational networks (e.g. Ahuja et al., 2012), change (e.g. Van de Ven \& Poole, 1995), and cross-sector collaborations (e.g. Bryson et al., 2006) to review research on TS change. Such a review should help in our quest to better understanding how a planned TS change may impact perceptions of interorganizational relationships over time. This section is broken down into three subsections. The first section (Section 2.2.1) provides an overview of four patterns of change in multi-organization systems. The second section, Section 
2.2.2, describes factors that may drive or shape TS change. Lastly, the third section summarizes and critiques the TS change literature, and identifies ways how this study will begin addressing identified gaps and challenges.

\subsubsection{PATTERNS OF TS CHANGE}

Although there has been a significant focus on TS change involving the formation of new and the dissolution of existing TS partnerships (Kim et al., 2006), there have been other patterns of TS change identified in the literature (Koka et al., 2006). Our review of literature suggests that there are four different ways that TSs may change: (1) a new organization may be added to an existing TS, (2) an old organization may be removed from an existing TS, (3) the relationship between two organizations within a TS may become stronger over time, and (4) the relationship between two organizations within a TS may become weaker over time.

The first two forms of TS change (i.e. the addition or subtraction of an organization from the TS) have been well explored in the strategic alliance and interorganizational network literature, and have seen a considerable amount of theory development. Strategic alliance researchers have explored alliance formation and dissolution (e.g., Phene \& Tallman, 2014), how this formation or dissolution may impact organizational and alliance performance (e.g. Gimeno, 2004), and, to a lesser extent, the impact of alliance formation and dissolution on alliance structures (Das \& Teng, 2000). The interorganizational network literature, on the other hand, has explored adding or removing an organization from the perspective of forming a new linkage or removing an old linkage between two organizations (Ahuja et al., 2012).

In contrast, few studies have explored the strengthening or weakening of linkages between organizations within a TS over time. Mariotti and Delbridge (2012, p.514) studied the strengthening and weakening of linkages within the European Motorsport industry by exploring 
potential ties, which refer to "embryonic relationships that have the opportunity of, and potential for, further development", and latent ties, which "are established ties that are currently inactive". They argue that strong ties become weak as more latent ties form, and weak ties can become strong when more potential ties are realized between existing partners (Mariotti \& Delbridge, 2012). Koka et al. (2006) theorize that changes in the environment can lead to changes in uncertainty and munificence, which refers to the extent to which resources available to a firm are plentiful or scarce, after taking into account the number of firms competing for those resources (Anderson \& Tushman, 2001). Koka et al. (2006) argue that interorganizational linkages are likely to strengthen when environmental shifts decrease uncertainty and munificence, and likely to weaken when environmental changes mean uncertainty decreases but munificence increases.

\subsubsection{FACTORS DRIVING AND SHAPING TS CHANGE}

As noted previously, research on TS change is scattered across several different literatures. While each of these pieces of literature approach TS change from a slightly different perspective, they have all contributed to our understanding of TS change by identifying their own set of factors that drive and/or shape TS change over time. Within this section, we identify and review four factors that may drive or shape how change unfolds in a TS over time: (1) agency, (2) opportunity, (3) inertia, and (4) degree of organization.

\subsubsection{AGENCY}

Agency refers to the focal actor's motivation and ability to shape relations, create beneficial links, dissolve unprofitable links or shape an advantageous structure (Ahuja et al., 2012). Focal actors may purposively choose or not choose to establish connections with certain other actors in

their TS, by forming or dissolving linkages between organizations within the TS, or by strengthening or weakening relationships (Emirbayer \& Mische, 1998; Burt, 2005). From the 
agency perspective, organizations within the TS may deliberately seek to change the TS so that they benefit in some way, such as accessing desirable TS resources (Das \& Teng, 2000). This research involves a TS consisting of three organizations that are differentially impacted by the external environment. The three organizations within the TS may, therefore, each be driven by an agency to change the TS in a way that benefits them, without considering how it will impact their TS partners.

\subsubsection{OPPORTUNITY}

An opportunistic perspective suggests that some TS changes are more likely than others, given the opportunities that present themselves. Opportunity reflects Blau's (1994) idea of the structural context of the situation. More specifically, Blau (1994) notes that actions (or changes in the case of this dissertation) are more or less likely to occur than others considering the structure of a particular system. While some architectures may limit an organization's ability to influence the system, others may offer advantages (Capaldo, 2007). Researchers have found that some dyadic behaviour is driven by convenience (Gulati \& Gargiulo, 1999) or by proximity (Rivera et al., 2010). For example, Rivera et al. (2010, p.105) argue that

"the most elementary proximity hypothesis is that interaction increases with geographic/physical propinquity. Being proximate is thought to encourage chance encounters and opportunities for interaction, which can lead to the formation of new relationships and the maintenance of existing ones."

This research involves a TS consisting of three organizations that are obligated to interact with each other based on government legislation. The organizations within the TS may, therefore, be driven by the opportunity to change the TS based on criteria set out by the government mandate. 


\subsubsection{INERTIA}

Inertia includes the pressures for persistence and change (Coleman, 1988) and refers to the durability of social structures as well as the social processes by which the principal actor's actions are influenced, directed, and constrained by norms and institutional pressures. In other words, TSs may not change because of routines or norms that develop over time. Persistence matters because the factors that impede or inhibit change in TSs may also be as relevant for TS change as those that enhance change (Kim et al., 2006). In their review of the network inertia literature, Kim et al. (2006) argued that inertia may be impacted by factors at the organizational level (e.g., organization age, size), dyadic level (e.g. network tie duration), network level (e.g. structural holes), and field level (e.g. institutional environment). As this dissertation case study involves three organizations that have existed for a long time, it is possible inertial structures have formed within the TS and that they may influence how the planned TS change may impact interorganizational relationships over time.

\subsubsection{DEGREE OF ORGANIZATION}

Roberts (2004) envisions the degree of organization within a TS as being related to the "tightness of the collaborative structure governing the collaborative parties" and conceptualizes TSs falling on a continuum of 'tightness', as shown in Figure 3. Largely dependent on the degree of organization (or tightness) of the TS, Roberts (2004) classifies four different types of TSs: coalitions, alliances, partnerships, and joint ventures. Coalitions are defined as engaging in a "non-trivial degree of coordinated activity over time" (Sabatier, 1998, p. 103). Partnerships are "a relationship in which [multiple groups] are jointly committed to the success of whatever process [they] are in" (Oshry, 2007, p. xxi). Alliances are "voluntary cooperative inter-firm agreements aimed at achieving competitive advantage for partners" (Das \& Teng, 2000, p. 33). 
Finally, joint ventures are defined as activities "... in which the operations of two or more firms are partially, but not totally, functionally integrated in order to carry out activities..." (Caloghirou, Ioannides \& Vonortas, 2003; OECD, 1986).

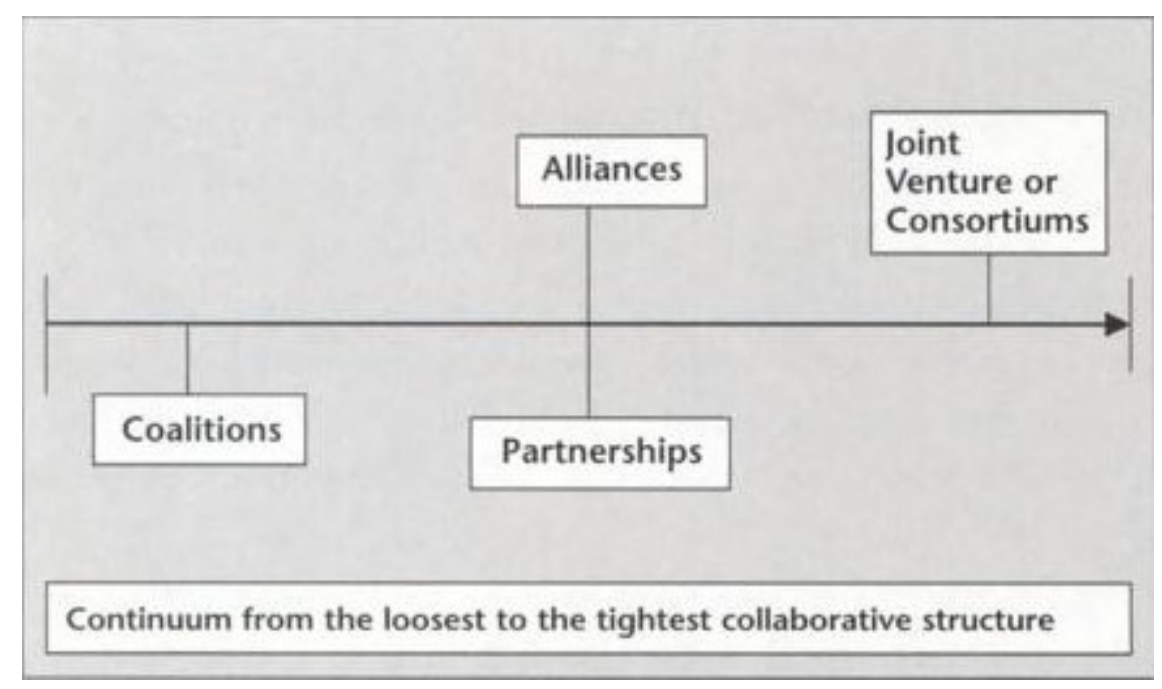

Figure 3: Degree of Organization within TSs (Roberts, 2004)

Coalitions, partnerships, alliances and joint ventures have different mechanisms that may impact their behavior, and thus shape how TS change unfolds over time. According to Roberts (2004), coalitions usually have the least structure and a low degree of organization compared to other TSs, often relying only on terms of reference and a decision-making process. Coalitions tend to be used for advocacy purposes. As a result, coalitions forgo a vision development process for a process for reaching agreement on objectives on an advocacy strategy.

Partnerships, according to Roberts (2004), are slightly more organized than coalitions in that they may have some documentation outlining measures to achieve a common goal. The term partnership is particularly favored by government entities (e.g., Allen, Juillet, Paquet \& Roy, 2001; Gazley, 2008) and can include anything from organized consultation processes to service delivery partnerships controlled by contractual agreement. 
Alliances, often termed strategic alliances in the management literature, involve at least two partner organizations that remain legally independent after the alliance is formed. They share benefits and managerial control over the performance of assigned tasks and make continuing contributions in one or more strategic areas, such as technology or production (Roberts, 2004).

Joint ventures demonstrate a high degree of organization: they have a clear purpose or a common goal, involve a tightly focused service or structure to share administrative functions, and have a formal agreement regarding partner contributions and commitment of resources (Roberts, 2004). Partner organizations remain autonomous, with separate decision-making boards and administration.

In this research, the TS under study involves three public sector organizations who are working collaboratively to deliver a service. While there was no formally agreed upon policy between partners, it was expected that all parties would sign a memorandum of understanding (MOU) before any change to the PMI process. An MOU is a signed document describing the general principles of ongoing cooperation, coordination, and alignment between signing parties. These facts would suggest that there is a moderate level of organization within the TS we are studying. Also relevant is the fact that there is evidence of a service delivery partnership controlled by contractual agreement. Based on Roberts' (2004) framework, it would appear therefore that the TS we are studying falls into the partnership group. In other words, we plan to explore how a planned TS change impacts perceptions of interorganizational relationships in a moderately organized TS. Given Brown's (1980) research on under-organized systems, the process of change in moderately organized TSs is likely to be different from the process of change in under- and over-organized TSs. 


\subsubsection{CRITIQUE OF TS CHANGE LITERATURE}

Despite the growing body of literature associated with TSs, there are still significant theoretical and empirical gaps in the TS change research. Below are three critiques of the TS change literature and ways that this study intends to address these shortcomings.

First, there is limited theoretical and empirical research exploring how TSs change over time (Ahuja et al., 2012). Kim et al. (2006) argue that the traditional assumption that organizations enter or exit partnerships based solely on the idea that a different partnership arrangement may increase value, rather than considering the difficulty in actually changing the partnership arrangement, has led to limited research on the process of TS change. This case study contributes to understanding the process of TS change by studying a TS and its interorganizational relationships before and after changes have been implemented.

Second, research on TS change is spread out across various literature (e.g., strategic alliance, networks, change, and cross-sector collaborations). While this could be considered a strength of the field (i.e. theoretical triangulation), most TS change scholars draw from only one or two literatures when conducting their research (Bryson et al., 2006). The implications of this are that the development of the TS change concept is currently compartmentalized. The thesis research, on the other hand, contributes more broadly to the TS change literature by drawing from numerous fields to develop a theoretical framework of how a planned TS change impacts perceptions of interorganizational relationships over time.

Third, there has been limited research exploring stakeholders' perceptions and how they may shape TS change over time. Although this review of the literature identified five factors that 
may impact TS change, none of them considered stakeholders' perceptions. This is unfortunate, as the organizational change literature has shown that workers' perceptions of the change may impact how change unfolds (Armenakis et al., 1993; Armenakis \& Bedeian, 1999), and the broader management literature has shown that 'sharedness' may impact group dynamics (Luo, 2005). Interestingly, however, there has been very little research on perceptions or 'sharedness' within an interorganizational context. Accordingly, this research contributes to the TS change literature by exploring 'sharedness' within a TS undergoing a planned change.

\subsection{STAKEHOLDER THEORY}

The stakeholder literature has theoretically and empirically explored the notion that interorganizational relationships may influence change in larger systems. Therefore, in this thesis we felt it appropriate to use stakeholder theory as the lens to connect the two key ideas in our model: planned TS change and the relationships between organizations within a TS undergoing such change.

According to Lewin (1947a), gatekeepers are individuals that hold the most power within a group and may use their power to influence how change unfolds over time. While gatekeeping theory may be helpful to understand how change unfolds in a group of individuals, there has been very little exploration of the theory in other settings (Shoemaker, Eichholz, Kim \& Wrigley, 2001).

The stakeholder literature, on the other hand, provides theory and empirical evidence supporting the notion that some organizations within a group of organizations may be more powerful than others and may use their power to influence how change unfolds in the system over time. This literature also suggests that, while interorganizational power may be a significant dimension of understanding how organizations are connected, there are other dimensions to interorganizational 
relationships which can be equally important to understanding interorganizational dynamics (Mitchell et al., 1997).

Accordingly, the purpose of this section of the literature review is to situate this dissertation research in the stakeholder literature and theoretically develop our conceptualization of interorganizational relationships. The first section defines who a stakeholder is to justify the use of stakeholder theory within this research. The second section outlines the three approaches to stakeholder theory taken in the literature in order to situate this research in the appropriate research stream. Third, the literature on stakeholder classifications is reviewed, and one stakeholder classification is selected for use in this research, as it classifies stakeholders based on their perceived interorganizational influence. It is within this section that we develop our conceptualization of interorganizational relationships. The literature that links the stakeholder classification selected to stakeholder action is reviewed in the fourth subsection in order to help us understand how certain stakeholders may drive other organizations to take action, while other stakeholders may restrain organizations from taking action. Finally, in the fifth subsection, we summarize and critique the stakeholder literature, and identify ways in which this study will begin addressing these gaps and challenges.

\subsubsection{WHAT IS A STAKEHOLDER?}

The term "stakeholder" has been used indiscriminately in the last two decades in both practitioner and academic literature (Mainardes et al., 2011). The term is popular with practitioner groups in business, governments, health-related entities, and even the media. Despite this widespread usage, many practitioners who adopt the term neither define the concept nor provide a clear description as to what constitutes a stakeholder or stakeholder behavior (Mainardes et al., 2011). Academics, on the other hand, tend to define the stakeholder concept so broadly that they 
have been unable to reach consensus on an accepted definition. The original use of the word stakeholder, coined in an internal memorandum at the Stanford Research Institute in 1963, referred to "those groups without whose support the organization would cease to exist" (Freeman \& Reed, 1983). Fifty years later, over 66 different definitions for the term stakeholder appear in the research literature (Bryson, 2004; Buchholz \& Rosenthal, 2005; Pesqueux \& Damak-Ayadi, 2005; Friedman \& Miles, 2006; Beach, 2008).

Although many of these definitions share great similarity, they differ on how they see the directionality of influence between stakeholder and organization: is it the stakeholder that influences the organization, is it the organization that influences the stakeholder, or is it both? For example, Eden and Ackermann (1998, p.117) define stakeholders as "people or small groups with the power to respond to, negotiate with, and change the strategic future of the organization" and Donaldson and Preston (1995, p.67) define stakeholders as "Groups or persons with legitimate interests." In these conceptualizations of a stakeholder, similar to that of its original use at the Stanford Research Institute in 1963, the stakeholders have an influential role over the organization. On the other hand, Clarkson's (1998, p.2) definition of stakeholders, "those persons or interests that have a stake, something to gain or lose as a result of its [the organization's] activities", suggests that organizations have influence over the stakeholders.

The co-existence of these two conceptualizations suggests that there may be bi-directional influence between stakeholder and organization. The literature offers multiple definitions for the term stakeholder to reflect this claim.

- "Any person, group or organization that can place a claim on the organization's attention, resources, or output, or is affected by that output" (Bryson, 2004, p. 27) 
- "Those individuals or groups who depend on the organization to fulfill their own goals and on whom, in turn, the organization depends" (Johnson \& Scholes, 2002, p. 206)

- "Any individual or group who can affect or is affected by the actions, decisions, policies, practices, or goals of the organization" (Carroll, 1996, p. 74)

- "An individual or group that has some kind of stake in what business does and may also affect the organization in some fashion" (Buchholz \& Rosenthal, 2005, p. 138)

- "Groups or individuals who can actually or potentially affect or be affected by the achievement of governance network outcomes" (Mitchell, Agle \& Wood, 1997, p. 869)

Each of these definitions use slightly different words to convey a similar message: stakeholders are individuals, groups, and/or organizations who may influence or may be influenced by another individual's, group's, and/or organization's resources (Bryson, 2004), strategies (Eden \& Ackermann, 1998), interests (Carroll, 1996), and outcomes (Mitchell et al., 1997).

Although the concept of stakeholder is commonly applied to the relationship between one organization and one stakeholder, stakeholders may also participate in TSs composed of multiple different parties (Sink, 1991; Boje \& Rosile, 2003; Worley \& Parker, 2011). In fact, Worley and Parker (2011) use the term 'multi-stakeholder sustainability network' to describe how the concept of TSs may be conceptually expanded from its focus on organizations to include stakeholderstakeholder and stakeholder-organization processes. Worley and Parker's (2011) research showed how governmental agencies, businesses, philanthropic organizations, NGOs, consulting firms, and private citizens tried to move from an under-organized and tacit set of ineffective relationships toward a structural collaboration to gain common benefits. They found that some stakeholders influenced the multi-stakeholder sustainability network (i.e. TS) more than others (Worley \& Parker, 2011). 
Thus, while the concept of a stakeholder may be applied to individuals, groups, and organizations influencing or being influenced by organizations, it may be more narrowly applied to those individuals, groups, and organizations influencing or being influenced by others within a TS. Given that this research seeks to increase our understanding of how planned change unfolds in a TS consisting of three organizations (i.e., police, hospital and social service agency), this research is more concerned with organizations within a TS than individuals, groups, or organizations external to a TS. Accordingly, we limit the field's broad conceptualization of a stakeholder for this research by defining a TS stakeholder as an organization within a TS that may influence or may be influenced by another organization within the same TS. Thus, within this case study, we view the police, hospital, and social service agency as TS stakeholders.

\subsubsection{APPROACHES TO STAKEHOLDER THEORY}

There are three approaches to stakeholder theory (Friedman \& Miles, 2006): normative, descriptive, and instrumental. The normative approach to stakeholder theory defines how businesses should operate, especially in relation to moral principles (Friedman \& Miles, 2006). From Freeman's (1998) perspective, organizations should not engage in relationships with stakeholders merely as a means of raising organizational performance. Rather, organizations should develop these relationships within a fair, ethical, and morally correct framework, where interests are not purely economic, and where both the actions of management as well as the results obtained are justified (Friedman \& Miles, 2006). Research carried out under this approach is normally related to business ethics and what organizations should do according to a moral standard (e.g., Kaler, 2002).

The descriptive approach to stakeholder theory seeks to describe and explain characteristics of the interactions between organizations and stakeholders (Friedman \& Miles, 2006). This 
perspective discusses issues relating to the nature of the firm, how managers act, and how managers think strategically about the involvement of stakeholders (Donaldson \& Preston, 1995). Researchers have used the descriptive approach to stakeholder theory as a lens to rank stakeholders in terms of organizational importance (Mitchell et al., 1997) or attribute meanings to each stakeholder (Jawahar \& McLaughlin, 2001). Research carried out under this approach is normally exploratory and seeking to understand how organizations and stakeholders are connected (Mainardes, Alves \& Raposo, 2011).

The instrumental approach to stakeholder theory explores how stakeholder management may be used to attain organizational performance objectives by contributing to a manager's strategic decision-making (e.g., maintaining certain stakeholder relationships) (Donaldson \& Preston, 1995; Jones \& Wicks, 1999). This approach relates primarily to the management of relationships within specific stakeholder groups (Freeman, 1984). Some researchers have used the instrumental approach to explain interorganizational relationships with stakeholders, particularize stakeholder interests, and raise managerial awareness of organizational decisions, processes, and policies based on the analysis of each stakeholder (Starik, 1994; Mitchell et al., 1997). Other researchers extend the instrumental approach to a higher level of analysis, suggesting organizational performance depends on networks of stakeholders (Rowley, 1997).

As the case study in this thesis describes how the relationships within three TS stakeholders influence and are influenced by planned changes that take place within a TS over time. Of the three possible approaches to stakeholder theory, this research is most likely to contribute to the descriptive approach to stakeholder theory. 


\subsubsection{STAKEHOLDER CLASSIFICATIONS}

Stakeholders have been classified based on the level and type of influence they exert on organizations and what Freeman (1994) calls "the principle of who or what really counts". Researchers argue that they can learn more about stakeholders by categorizing and comparing similar stakeholders (Freeman \& McVea, 2001). The purpose of this subsection is to provide the reader with a general understanding of how researchers have classified stakeholders. Stakeholder classifications are relevant to this research as some classification schemes may suggest alternative ways to conceptualize interorganizational relationships within TSs.

Researchers have used all three of the approaches to stakeholder theory (i.e., descriptive, instrumental and normative) discussed in the previous subsection to develop classification schemes for stakeholders. Descriptive stakeholder classifications focus on the relationship between stakeholder and organization. For example, Kochan and Rubinstein (2000) suggested that all stakeholders should be categorized by the way they influence organizations and listed three criteria to identify the salience of potential stakeholders in terms of the organization:

1) The extent to which they contribute valuable resources to the enterprise;

2) The extent to which they put these resources at risk and would realize costs were the organization to fail or their relationship with the organization terminate; and

3) The power they have over the organization.

Pelle-Culpin (1998) also uses the descriptive approach to classify stakeholders based on their relationship to external entities. Pelle-Culpin (1998) offers the distinction between institutional stakeholders (i.e., those involved in laws, regulations, interorganizational entities, plus professional organizations that may be specific to a given industry), economic stakeholders (i.e. actors operating in the markets of the organization in question), and ethical stakeholders emanating 
from ethical and political pressure groups (i.e. a group whose figuration may be more difficult to define).

Next, instrumental stakeholder classifications focus on performance criteria. An example of a performance oriented classification is provided by Atkinson, Waterhouse, and Wells (1997). They theorize that companies exist to achieve their primary objectives, which are controlled by the organization's owners, and secondary objectives, which are dictated by the primary objectives and represent operational targets (ibid). Atkinson and colleagues (1997) categorize stakeholders into two groups: environmental (customers and community) and process (employees and suppliers). They further suggest that each stakeholder group is evaluated by the owners (i.e., shareholders) on the basis that they contribute to the primary and secondary objectives (ibid).

The final type of stakeholder classification, based on the normative approach to stakeholder theory, categorizes stakeholders based on how they ought to operate. Kaler (2002), for example, uses a business ethics perspective to classify three types of stakeholders: (1) claimants, stakeholders with some claim on the services of an organization; (2) influencers, stakeholders with only a capacity to influence the workings of the business; and (3) combinatory, stakeholders with both of these requirements. Kaler (2002) argues from a business ethics perspective; stakeholder management has to be about improving the moral conduct of businesses by managing stakeholders to improve more than just the organization's interests. He concludes that, for the purposes of business ethics, the only real type of stakeholder is a claimant, as both influencer and combinatory have the potential to entertain morally neutral strategic considerations (Kaler, 2002).

A second example of stakeholder classifications, based on the normative approach to stakeholder theory, was conceptualized by Mitchell, Agle and Wood (1997). They argue that stakeholders possess or are attributed to possess one, two, or all three of the following attributes: 
(1) the stakeholder's power to influence the organization, (2) the legitimacy of the stakeholder's relationship with the organization, and (3) the urgency of the stakeholder's claim on the firm (Mitchell et al., 1997). Stakeholder power exists when Stakeholder A can get Stakeholder B to do something that Stakeholder B would not have otherwise done (Mitchell et al., 1997, p. 865). Stakeholder legitimacy represents the belief that the actions of a stakeholder are desirable or appropriate within the organization's accepted norms and values (Mitchell et al., 1997, p. 866). Stakeholder urgency includes both criticality and time urgency, such that a stakeholder's claim is considered to be urgent when it is critical and/or a delay in response is unacceptable (Mitchell et al., 1997, p. 867).

The utility of this classification scheme comes from its simplicity in determining stakeholder salience. Mitchell et al. (1997) define salience as "the degree to which managers give priority to competing stakeholder claims" (p. 869). They argue that stakeholders with more attributes (i.e., power, legitimacy, urgency) are more salient to managers than stakeholders with fewer attributes and that managers should respond to salient stakeholders before responding to non-salient stakeholders (Mitchell et al., 1997). Mitchell, Agle and Wood's (1997) power, legitimacy, and urgency classification scheme has been widely referenced and empirically tested (e.g., Agle, Mitchell \& Sonnenfeld, 1999; Harvey \& Schaefer, 2001; Parent \& Deephouse, 2007; Winn \& Keller, 2001; Howard, Vidgen \& Powell, 2003).

As Mitchell et al.'s (1997) theory classifies stakeholders based on how organizations are perceived to be connected, their three dimensions of stakeholder attributes will be used in this research to conceptualize interorganizational relationships. For the purpose of this research, TS stakeholder relationships refer to the interorganizational coordinating mechanisms of power, legitimacy, and urgency within a TS. These three TS stakeholder attributes may be defined in the 
context of this research as follows: (1) power refers to a TS stakeholder's ability to influence other TS stakeholders, (2) legitimacy refers to the generalized perception or assumption that the actions of a TS stakeholder within the TS are desirable or proper, and (3) urgency refers to the degree to which TS stakeholder claims call for immediate action. With the addition of this terminology, we can say that the primary objective of this research is to develop a framework describing how a planned TS change impacts perceptions of TS stakeholder relationships over time.

Mitchell et al.'s (1997) theory of stakeholder power, legitimacy, and urgency has also been explored in the context of change. In an interesting post-hoc case study analysis of multiple organizations involved in the Pan AM games, Parent and Deephouse (2007) classified stakeholders based on managerial perceptions of the stakeholder's power, legitimacy, and urgency. Parent and Deephouse (2007) found that the classifications for certain stakeholders changed over time (i.e. the manager perceived a stakeholder possessing urgency in the beginning and then not possessing urgency later on). Although the focus of Parent and Deephouse's (2007) study was not on the concept of change, it does provide empirical support that perceptions of stakeholder's power, legitimacy, and urgency can change over time. The next section provides a brief review of how researchers have extended Mitchell et al.'s (1997) framework by linking power, legitimacy, and urgency to stakeholder action. This gives us a better understanding of how perceptions of power, legitimacy and urgency of the various TS stakeholders in our case study (i.e., police, hospital, and social service agency) may either drive or restrain TS stakeholders from taking action leading to change.

\subsubsection{STAKEHOLDER ACTION}

Researchers have extended Mitchell et al.'s (1997) research by theorizing linkages between stakeholder action and the possession of stakeholder: (1) power (Frooman, 1999), (2) legitimacy 
(den Hond \& de Bakker, 2007), and (3) urgency (Rowley \& Moldoveanu, 2003; Bundy et al., 2013). Although stakeholder action is not explicitly defined in the literature, stakeholders act to enhance interests or preserve identities (Rowley \& Moldoveanu, 2003). Interest-based action, on the one hand, occurs when a stakeholder mobilizes to protect their interests (Tilley, 1978). Identity-based action, on the other hand, refers to stakeholder actions that are driven by the need to retain a stakeholder's affiliation to a group (Rowley \& Moldoveanu, 2003). For example, an individual who feels a strong affiliation with an environmental activist group, such as Greenpeace, will reinforce his or her identity as an environmentally conscious person by repeatedly participating in the group's activities, even though these activities are not expected to protect or enhance their collective interests (Abrahamson \& Fombrun, 1994). The next three subsections describe how the possession of stakeholder attributes may relate to action.

\subsubsection{STAKEHOLDER POWER}

Frooman (1999) extends the notion of stakeholder power by developing a framework that categorizes perceptions of the relative power between organization and stakeholder. Drawing from conservation of resources theory, Frooman (1999) envisions four types of resource dependency relationships (see Figure 4): (1) stakeholder dependent on organization for resources, (2) organization dependent on stakeholder for resources, (3) organization dependent on stakeholder

for resources and stakeholder dependent on organization for resources, and (4) no dependency for resources between organization and stakeholder. Within this framework, Frooman (1999) argues that stakeholders are more powerful than organizations when organizations are dependent on them for resources but they are not reliant on the organization for resources. Frooman (1999) concludes that stakeholders with varying levels of power will take different actions to influence organizations. In the context of this case study on TS change, Frooman's (1999) framework 
suggests that the actions TS stakeholders may take with respect to TS change may be based on the relative power (i.e. resource relationship) each TS stakeholder is perceived to possess.

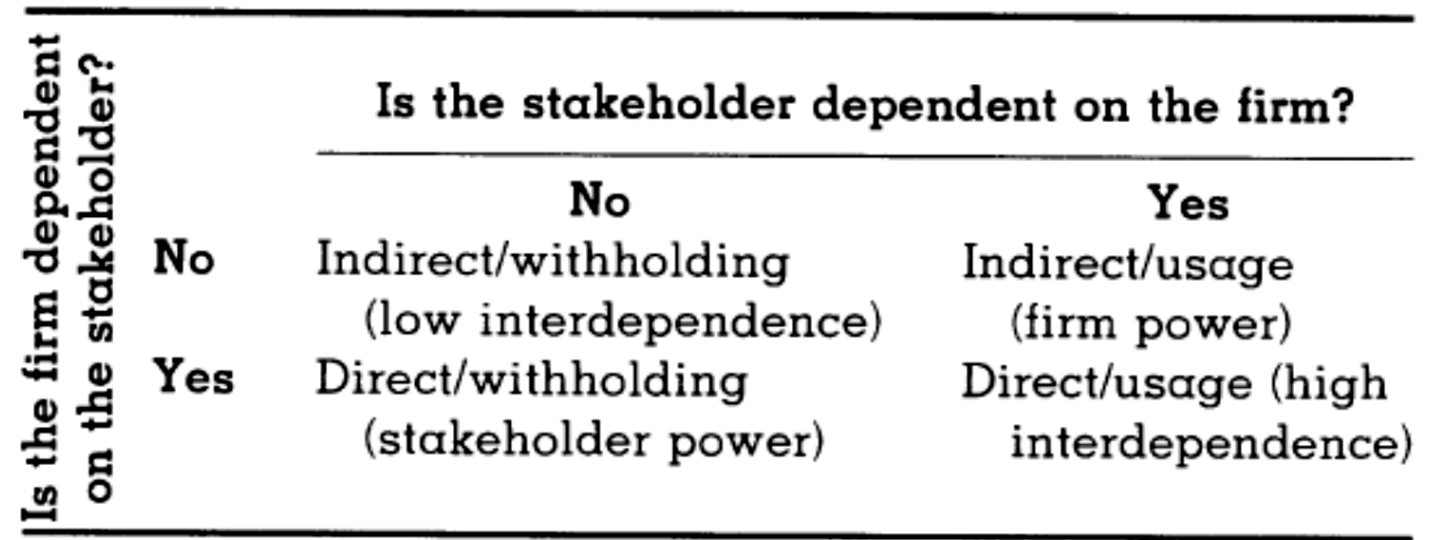

Figure 4: Frooman's (1999) Typology of Influence Strategies

Supporting Frooman's (1999) theorization of power in terms of resource dependence, previous studies exemplify how stakeholder behavior may be dependent on the resources the stakeholders have. In a study of Amoco Corporation, wealthier stakeholder groups were found to influence the decision by Amoco to respond to activist pressure (Hoffman, 1996). Similarly, a handful of papers in the environmental justice literature have looked at the effect of local community pressure on firm behavior and found, in particular, that wealthier constituencies tend to have less polluting firms within their communities (Arora \& Cason, 1999). Although power has been linked to action (Frooman, 1999) and power has been well documented as a key factor in organizational change (e.g., Pettigrew, 1979; Pfeffer, 1981), limited research could be identified that links interorganizational power, or actions associated with interorganizational power, to TS change. In this research, we seek to establish: (1) which of the three TS stakeholders are perceived to have power within the TS, (2) how these perceptions of power relate to planned TS change, and (3) how perceptions of power within the TS change over time. Frooman's (1999) research would 
suggest that the TS stakeholder with the most power might have more influence on how planned change unfolds in a TS than their counterparts with less power.

\subsubsection{STAKEHOLDER LEGITIMACY}

Den Hond and de Bakker (2007) draws on the social movement literature and change theory to explore how stakeholder legitimacy influences corporate social change. Den Hond and de Bakker (2007) argue that stakeholders may invoke moral or pragmatic legitimacy in order to influence larger systems (Greenwood et al., 2002; Lounsbury \& Glynn, 2001). Moral legitimacy refers to an evaluation of whether the organization "effectively promote[s] societal welfare, as defined by the audience's socially constructed value system” (Suchman, 1995, p. 579), whereas pragmatic legitimacy “rests on the self-interested calculations of an organization's most immediate audiences" (Suchman, 1995, p. 578). Den Hond and de Bakker (2007) suggest that stakeholders with different types of legitimacy (e.g., moral or pragmatic) will take different actions to influence organizations.

This literature review has also found empirical research to support the links between moral legitimacy and stakeholder action. For example, a stakeholder with moral legitimacy may take corporate symbols out of context and try to convince the public at large that the firm belongs to some morally disfavored taxonomic category (Bennett, 2004). Although stakeholder legitimacy has been linked to institutional change (den Hond \& de Bakker, 2007), no research could be uncovered connecting perceived stakeholder legitimacy to TS change, a shortcoming that will be addressed in this research. In this study, we seek to establish: (1) which of the three TS stakeholders are perceived to have moral legitimacy within the TS, (2) how these perceptions of legitimacy relate to planned TS change, and (3) how these perceptions of legitimacy within the TS change over time. The above literature would support the idea that TS stakeholders with higher 
levels of perceived moral legitimacy might have a greater influence on how planned change unfolds in a TS than their counterparts with less perceived moral legitimacy.

\subsubsection{STAKEHOLDER URGENCY}

Rowley and Moldoveanu (2003) use social movement and social identity theories to form a link between stakeholder urgency and stakeholder action. Rowley and Moldoveanu (2003, p.204) argue that the literature reveals the assumption that interest intensity, defined as " $a$ stakeholder group's degree of discontent or feeling of urgency", is the primary condition driving it to take action to influence another firm. While the theoretical link between urgency and change has not been firmly established (Rowley \& Moldoveanu, 2003), urgency has been shown empirically to positively influence outcomes. For example, Andersson and Bateman (2000) found that stakeholder groups who frame their environmental concerns as urgent issues are more likely to achieve favorable outcomes than those who do not frame their concerns as urgent. The psychology literature also provides some evidence that the urgency of a claim will influence the likelihood of response. In particular, time pressures have been shown to make decision-makers more prone to take action (Dror, Busemeyer \& Basola, 1999).

Even though an urgent claim may trigger the desire of the stakeholder to take action, Rowley and Moldoveanu (2003) argue that it may not necessarily influence the stakeholder's ability to act: "the desire to act and actual group mobilization are separated by the cost of organizing for such action” (p. 208). To overcome these costs, Rowley and Moldoveanu (2003) suggest, stakeholders are more likely to act when multiple stakeholders are willing to engage in action simultaneously, and thus are ready to share the "costs" associated with change. For example, Knight and Greenberg (2002) described the situation where multiple activist groups teamed up in the anti-sweatshop movement to exert pressure on major brand producers in the 
apparel and shoe industry. Multiple groups working together suggest the presence of an important issue that needs attention and has been theorized to associate with higher levels of firm responsiveness, "the degree to which a firm is willing to provide a thoughtful response to stakeholder concerns or commit to continued work on the issue" (Bundy et al., 2013, p. 352). In this research, we seek to establish: (1) which of the three TS stakeholders are perceived to have urgency within the TS, (2) how these perceptions of urgency relate to planned TS change, and (3) how these perceptions of urgency within the TS change over time. The above review of the literature would support the idea that TS stakeholders perceived to have greater urgency would have greater influence on how planned change unfolds in a TS than their counterparts perceived to have less urgency.

\subsubsection{CRITIQUE OF STAKEHOLDER LITERATURE}

This review of the stakeholder literature identifies numerous theoretical and empirical gaps that will be addressed by this research. One of the major issues with the stakeholder field, previously identified by Key (1999), is the notion that stakeholder theory incorrectly approaches the environment as something static that does not change. This research addresses this criticism by exploring how a planned TS change impacts perceptions of TS stakeholder relationships over time.

The next criticism relates to how researchers have studied the relationships between organization and stakeholder. Many researchers (e.g., Stoney \& Winstanley, 2001; Comeche \& Loras, 2010) argue that empirical treatment of these relationships has been oversimplified. Stoney and Winstanley (2001) label stakeholder theory as a political pluralism theory. Adopting a Marxist criticism of pluralism, these authors argue that stakeholder theory supplies an excessively simplistic conceptualization of relationships. Stoney and Winstanley (2001) argue, for example, 
considering power as a good that may be negotiated between the organization and stakeholders is very limited in its explanations of the means by which different stakeholders and organizations interact. Without the consideration of multiple divergent interests, Comeche and Loras (2010) argue, stakeholder theory may easily be subverted to a unitary concept. This research takes numerous strategies to addresses this critique. First, this research explores stakeholders from multiple perspectives: power, legitimacy, and urgency. Second, this case study has been designed to capture data on these multiple perspectives using interviews, which allow for an in-depth investigation of each of these stakeholder attributes. Third, this research collects data from three TS stakeholders, not just one, to get a better understanding of the TS stakeholder relationships. Finally, data is collected on TS stakeholder relationships at two separate time periods, which allow for a detailed investigation how a planned TS change may impact perceptions of TS stakeholder relationships over time.

Lastly, the stakeholder literature is inconsistent when discussing how stakeholders may influence organizations. As noted in the discussion of the definition of stakeholder, the stakeholder model represents the relationship between the stakeholders and the organizations by means of a bi-directional influence. In other words, researchers assume that the relationships between stakeholders are reciprocal given that each may impact the other regarding losses and gains as well as rights and duties (Evan \& Freeman, 1988). Other research in the area suggests, however, that not all relationships are equal and that the intensity of interaction in one direction might be quite different from the other direction depending on the stakeholder relationships (Post, Preston \& Sachs, 2002; Phillips, 2003). This latter argument (i.e. not all relationships are equal) is consistent with Lewin's (1947a) gatekeeping argument, which suggests that not all individuals in a group are 
equally important. This research addresses this critique by exploring both directions of influence on each TS stakeholder (i.e., organization perspective, stakeholder perspective). 


\section{CHAPTER 3: DEVELOPMENT OF RESEARCH OBJECTIVES AND QUESTIONS}

Miles and Huberman (1994) argue that an orienting framework helps the researcher set boundaries for the research, and develop research questions that guide the data collection and analysis (see Chapter 4). This section of the thesis uses the literature reviewed in Chapter 2 to develop research objectives and an orienting framework to guide the research. The overarching research objectives are first presented in Section 3.1. This is followed by the more specific research questions in Section 3.2.

\subsection{RESEARCH OBJECTIVES}

This thesis has two overarching objectives. The first is to develop a framework describing how a planned TS change impacts perceptions of TS stakeholder relationships over time (Research Objective 1). The second is to investigate whether the 'sharedness' concept provides a useful lens through which to understand the impact of planned TS change on perceptions of TS stakeholder relationships (Research Objective 2). These objectives are discussed in this section as follows. We first define what we mean by 'sharedness' (Section 3.1.1), a key construct in our framework describing how a planned TS change impacts perceptions of TS stakeholder relationships over time. This is followed by the development of constructs and linkages within our preliminary orienting framework (Section 3.1.2).

\subsubsection{CONCEPTUALIZING 'SHAREDNESS' WITHIN TSs}

A key goal of this study is to explore how 'sharedness' across organizational boundaries may be impacted by planned TS change. The idea of 'sharedness' within a TS has rarely been explored, and there is no pre-existing framework for conceptualizing the idea of 'sharedness' across organizational boundaries within TSs. The literature reviewed in Chapter 2 does, however, offer 'pockets' of theory that might aid in the development of this concept. 
'Sharedness' has been defined as "the degree to which cognitions, preferences, identities, etc. are shared and are being shared within groups" (Tindale \& Kameda, 2000, p. 123). The 'sharedness' concept derives from two ideas: (1) groups of individuals can be conceptualized as information-processing systems, and (2) the degree to which perceptions are shared and are being shared within the group affects group dynamics (Tindale \& Kameda, 2000). In this study, TS stakeholders are conceptualized as groups of employees who interact across organizational boundaries within a TS. 'Sharedness' logic would then imply that the degree to which perceptions are shared within each group of employees (i.e. Hospital, Police, Social Service Agency) and between the different groups of employees across organizational boundaries will affect the dynamics of multi-organization collaboration, and potentially TS change initiatives (Halinski \& Duxbury, 2015). The extent to which this logic holds is examined in this research study.

As 'sharedness' is viewed as a group phenomenon (i.e. information can be shared or hidden from the group), it is commonly conceptualized as a continuum (Kerr \& Tindale, 2004). On one end of the continuum, perceptions are completely shared within the group, while on the other end, perceptions are held by a single member and hidden from the group. This conceptualization, however, de-emphasizes the notion that a pair of individuals within a larger group may share a perception that is not held by the entire group. The broader management literature has found that dyadic partnerships within larger groups/systems of organizations may share similar perceptions that end up driving them to take action against the larger system (Rowley \& Moldoveanu, 2003). This conceptualization of 'sharedness' at the individual level is expanded to the level of the TS stakeholder in this thesis, as we examine the extent to which pairs of TS stakeholders, as well the entire TS, may share similar or hold different perceptions. 

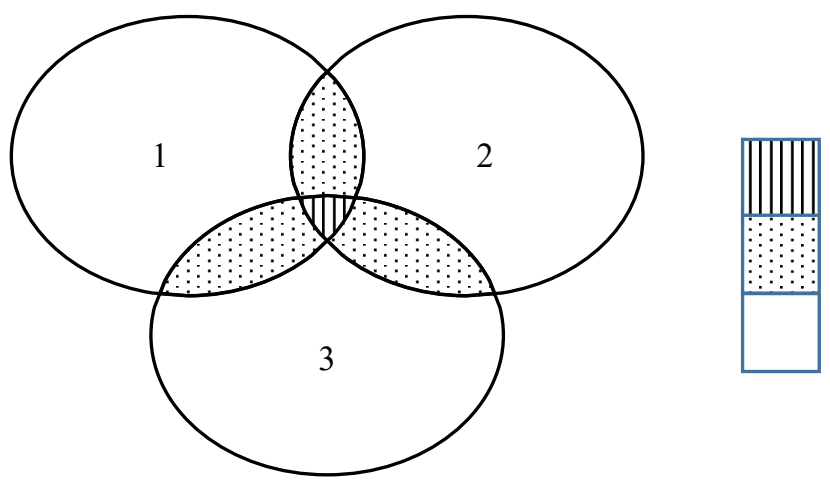

Common Perceptions

Shared Perceptions

Unique Perceptions

Figure 5: Conceptualizing ‘sharedness' with Common, Shared, and Unique Perceptions

Figure 5 illustrates our conceptualization of 'sharedness', which we define as the degree to which perceptions of the same objective reality are shared between and among TS stakeholders within the same TS. Circles 1, 2, and 3 represent the perceptions held by three different TS stakeholders within the same TS ${ }^{1}$. In this thesis, we examine 'sharedness' within the TS based on two sets of perceptions: (1) perceptions of power, legitimacy, and urgency within the TS, and (2) perceptions of desired and observed changes within the TS.

There are two aspects of 'sharedness' that are important to this thesis: the degree of consensus and intensity of the overlapping views. The degree of consensus refers to which partners have similar views. Based on a three TS stakeholder framework, there are three possible consensus states of 'sharedness': common, shared, and unique. We define these as follows. Common Perceptions occur when all TS stakeholders have the same perspective on a particular topic (e.g., TS stakeholder relationships, change). Shared Perceptions occur in TSs with three or more TS stakeholders when two of the TS stakeholders have the same view on a particular topic but this view is not shared across the third TS stakeholder. Unique Perceptions occur when the views of

\footnotetext{
${ }^{1}$ A similar figure could be used to describe a TS of 2 or more organizations. We use three organizations in the figure to be consistent with the number of organizations participating within the TS in the dissertation research.
} 
a TS stakeholder do not overlap with any other TS stakeholder. The intensity of 'sharedness' refers to the extent that views from different TS stakeholders overlap. Situations where there are a large amount of overlap between TS stakeholders are considered strong, whereas situations with a little amount of overlap between TS stakeholders are considered weak. The operationalization of these two terms are discussed in Section 4.7.1.2.

\subsubsection{CONCEPTUALIZING TRANSORGANIZATIONAL CHANGE}

The primary research objective of this thesis is to develop a framework describing how a planned TS change impacts perceptions of TS stakeholder relationships over time. The preliminary conceptual framework that will guide the investigation of this objective is presented in Figure 6 below. In this thesis, the guiding framework is applied to a TS consisting of three TS stakeholders.

The orienting framework consists of three different constructs: (1) Perceptions of TS Stakeholder Relationships, (2) Perceptions of Desired TS Changes, and (3) Perceptions of What Has Changed in the TS over time. Perceptions of TS Stakeholder Relationships refers to each TS stakeholder's perceptions of their own and their TS partners' power, legitimacy, and urgency within the TS. Perceptions of Desired TS Changes refers to what each TS stakeholder perceives needs to change within the TS. Perceptions of What Has Changed in the TS refers to each TS stakeholder's perceptions of what has changed in the TS between Time 1 (pre-change) and Time 2 (six months after the introduction of the planned TS change). 
Figure 6: Orienting framework describing how a planned TS change impacts perceptions of TS stakeholder relationships over time

\section{Time 1}

Perceptions of TS Stakeholder Relationships

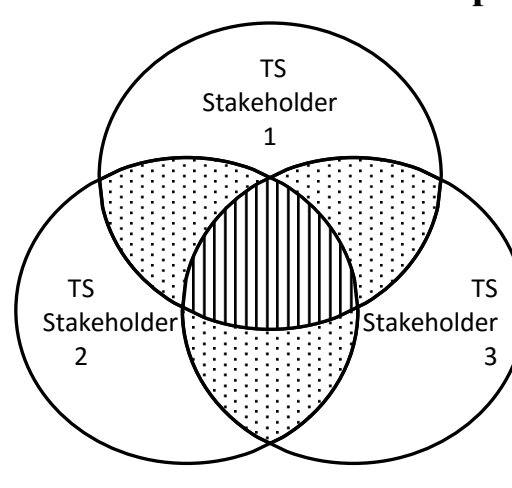

Time 2

Perceptions of TS

Stakeholder Relationships
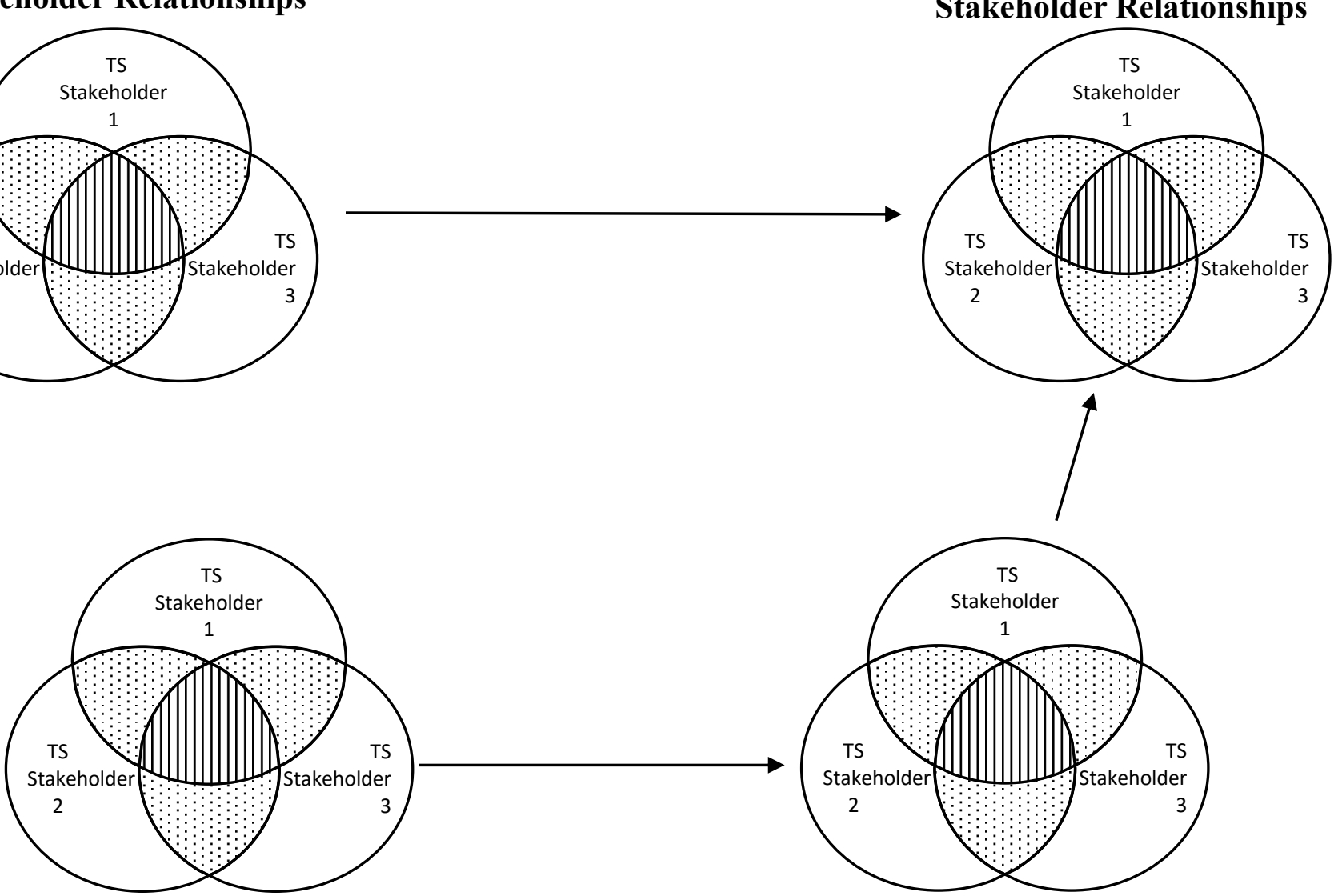

Perceptions of Desired TS Changes

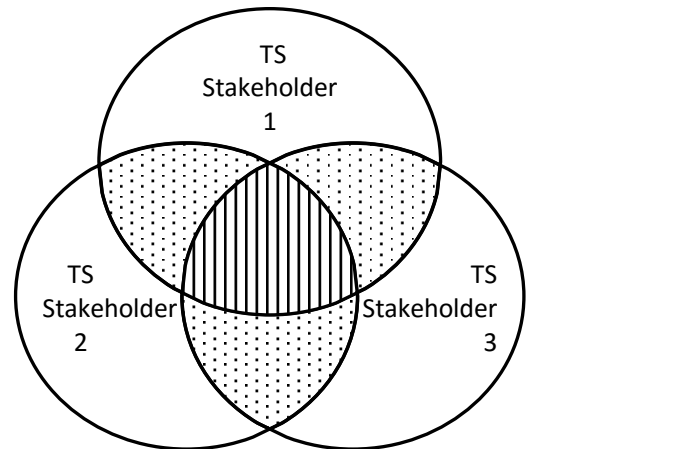

Perceptions of What Has Changed in the TS 
It should be noted that the guiding framework treats the time period in between Time 1 and Time 2 as a "black box". Although there are likely to be many important events taking place during this time interval (e.g., discussions, negotiations, decision-making, trial and error changes), the focus of this research is on how a planned TS change impacts perceptions of TS stakeholder relationships over time. The decision to explore changes in a construct (i.e. Perceptions of TS Stakeholder Relationships) at two separate times is consistent with Van de Ven and Poole's (2005) Approach 2 for studying change.

The description of the conceptual framework will begin by justifying the constructs underpinning the framework of how a planned TS change impacts perceptions of TS stakeholder relationships over time, as represented in Figure 6 . This is followed by a discussion of the suspected relationships between the constructs.

\subsubsection{PERCEPTIONS OF TS STAKEHOLDER RELATIONSHIPS}

Perceptions of TS Stakeholder Relationships refers to each TS stakeholder's perceptions of the extent to which they and the other TS stakeholders in the TS possesses power, legitimacy, and urgency within the TS. This view of relationships is consistent with Mitchell et al.'s (1997) stakeholder classification.

Stakeholder theory identifies three attributes that can describe relationships between TS stakeholders: power, legitimacy, and urgency (Mitchell et al., 1997). Stakeholder theory suggests that TS stakeholders who have critical resources, to which others are dependent, are perceived to have power within the TS (Frooman, 1999). Resources that are linked to power may include money (Hoffman, 1996), access to third party resources (McCarthy \& Zald, 2001), access to networks (Bovaird, 2005), infrastructure (Arora \& Cason, 1999), organizational learning (Roome \& Wijen, 2006), and materials and symbols (Mitchell et al., 1997). The resource-based perspective 
would suggest that an organization is perceived to have power when it can leverage the resources it has to make another group do what they want even if that group does not want to (Mitchell et al., 1997).

Stakeholder legitimacy has been theorized to be the generalized perception that the actions of a stakeholder are desirable, proper or appropriate within some socially constructed system of norms, beliefs, and values (Suchman, 1995). Stakeholders with legitimacy have a greater ability to cause harm to other organizations than illegitimate stakeholders (Rowley, 1997). This has frequently been recognized in the stakeholder literature as a critical attribute linking a stakeholder to an organization (Phillips, 2003; Driscoll \& Crombie, 2001; den Hond \& de Bakker, 2007)

Stakeholder urgency has been defined as the degree to which stakeholder claims call for immediate action (Mitchell et al., 1997). There are two components to stakeholder urgency: time sensitivity and criticality. Time sensitivity refers to the degree to which managerial delay in attending to the claim or relationship is unacceptable to the stakeholder, while criticality refers to the importance of the claim or the relationship to the stakeholder (Mitchell et al., 1997, p.869). Even though urgency and its theoretical linkage to time have been shown to relate to organization's perceptions of salient stakeholders (Agle et al., 1999), it is frequently overlooked by researchers (Mosakowski \& Earley, 2000; Driscoll \& Starik, 2004). This shortcoming is rectified in this research.

\subsubsection{PERCEPTIONS OF DESIRED TS CHANGES}

Perceptions of Desired TS Changes refers to what each TS stakeholder perceives needs to change within the TS, and taps into what Armenakis and Bedeian (1999) refer to as the "content" issues of change. Described as the substance of contemporary changes, the content of a change has been studied by researchers defining factors that comprise the targets of both successful and 
unsuccessful change efforts and how these factors relate to overall effectiveness (Armenakis \& Bedeian, 1999). Examples of factors that have been investigated include alternative strategic orientations, organizational structures, and performance-incentive systems (Burke-Litwin, 1992; Vollman, 1996).

\subsubsection{PERCEPTIONS OF WHAT HAS CHANGED IN THE TS}

Perceptions of What has Changed in the TS refers to each TS stakeholder's perceptions of what has changed in the TS post the introduction of planned change to the TS (i.e. between Time 1 and Time 2). Armenakis and colleagues (1999) have observed that most theoretical models of the change process (e.g., Judson, 1991; Kotter, 1995; Galpin, 1996) support Lewin's (1946a) conceptualization of the change process including three steps: unfreezing, moving and refreezing. Conceptually then, perceptions of what has changed in a TS should be measured in the refreezing stage of change. In this thesis, we assume that the TS is in the refreezing stage of change six months after the introduction of the planned TS change. Behaviors of possible interest may be related to consolidating and institutionalizing (Judson, 1991), publicizing the connection between the change effort and success (Kotter, 1995), and measuring, reinforcing and refining the change (Galpin, 1996).

\subsubsection{RELATIONSHIP BETWEEN PERCEPTION OF TS STAKEHOLDER RELATIONSHIPS AT TIME 1 AND TS STAKEHOLDER RELATIONSHIPS AT TIME 2}

The conceptual framework posits that Perceptions of TS Stakeholder Relationships at Time 1 relates to Perceptions of TS Stakeholder Relationships at Time 2. This theoretical linkage is supported by theory arguing that interorganizational relationships within a TS tend to be sustained over time (Peteraf, 1993), and that institutional arrangements and cultural orientations that characterize these business systems are usually very stable (Bachmann, 2003). Moreover, the 
public sector literature also supports the idea that cross-sector relationships are only likely to change in extreme conditions of sector failure, where single-sector efforts to solve a public problem are tried and fail (Bryson et al., 2006). This may explain why the stakeholder literature has provided little evidence to suggest that perceptions of power, legitimacy, and urgency change over time (see exception, Parent \& Deephouse, 2007).

\subsubsection{RELATIONSHIP BETWEEN PERCEPTIONS OF DESIRED TS CHANGES AT TIME 1 AND PERCEPTIONS OF WHAT HAS CHANGED IN THE TS AT TIME 2}

The conceptual framework posits that Perceptions of Desired TS Changes at Time 1 can affect Perceptions of What Has Changed in the TS at Time 2. Armenakis and Bedeian (1999) note that managerial actions taken in response to content considerations (i.e., desired changes) primarily result in two types of perceptions: perceptions in line with intentional outcomes and perceptions in line with unintentional outcomes. Intentional outcomes are the expected results from a change initiative and most often related to increased operational efficiency and effectiveness (Armenakis \& Bedeian, 1999). Numerous researchers (e.g., Judson, 1991; Kotter, 1995; Galpin, 1996) have conceptualized intended outcomes by modeling step-wise linkages between 'planning desired changes' and 'consolidating and institutionalizing the new state'.

\subsubsection{RELATIONSHIP BETWEEN PERCEPTIONS OF WHAT HAS CHANGED IN THE TS AT TIME 2 AND PERCEPTIONS OF TS STAKEHOLDER RELATIONSHIPS}

\section{AT TIME 2}

The conceptual framework posits that Perceptions of What Has Changed in the TS at Time 2 can affect Perceptions of TS Stakeholder Relationships at Time 2. As TSs change, TS resources are also likely to change (Feldman, 2004). Changing the network's distribution of resources may reduce a TS stakeholder's resource dependence on their partner and, according to Frooman's 
(1999) conceptualization of power, may reduce the partner's perceived power. Changing how TS stakeholders access TS resources may also impact the perceived legitimacy of a TS stakeholder (Lounsbury \& Glynn, 2001). Lastly, assuming that a TS stakeholder's access to certain resources was originally viewed as an urgent concern for that stakeholder, changing how they access resources may also reduce the TS stakeholder's urgent concerns. The above theory supports the inclusion in our model of a link between perceptions of what has changed in the TS and perceptions of TS stakeholder relationships at Time 2.

\subsection{RESEARCH QUESTIONS}

In exploratory research, an initial set of research questions helps focus data collection and constrain observations (Miles \& Huberman, 1994). The literature review and conceptual development given in the previous sections were used to generate a number of research questions that are organized by research objective in the subsections below.

\subsubsection{RESEARCH OBJECTIVE ONE: Develop a framework describing how a planned TS change impacts perceptions of TS stakeholder relationships over time}

The overarching objective of this research is to develop a framework describing how a planned TS change impacts perceptions of TS stakeholder relationships over time. Consistent with earlier conceptualizations of stakeholders (Mitchell et al., 1997) and change (Van de Ven \& Poole, 2005; Armenakis \& Bedeian, 1999), this research explores the TS change process by examining how three different TS stakeholders perceive relationships and TS change (i.e., desired changes, perceived changes) at two points in time. This approach enables the researcher to compare how TS stakeholders' perceptions of the TS stakeholders and the change itself held prior to the implementation of planned TS changes to their perceptions six months after the planned changes were implemented. This is done by seeking answers to the following research questions. 
1. How do TS stakeholders' perceptions of (a) power, (b) legitimacy, and (c) urgency within the TS before the implementation of planned change compare to their perceptions of (a) power, (b) legitimacy, and (c) urgency within the TS six months after the implementation of planned TS change?

2. How do TS stakeholders' perceptions of what needs to be changed with the TS compare to their perceptions of what has changed within the TS six months after the implemented of planned TS change?

3. How might TS stakeholders' perceptions of what has changed within the TS impact their perceptions of (a) power, (b) legitimacy, and (c) urgency within the TS six months after the implemented of planned TS change?

\subsubsection{RESEARCH OBJECTIVE TWO: Does the 'sharedness' concept provide a useful lens through which to understand the impact of planned TS change on perceptions of TS stakeholder relationships?}

The secondary objective of this research is to explore whether the 'sharedness' concept provides a useful lens through which to understand the impact of planned TS change on perceptions of TS stakeholder relationships. Whereas the first objective targets how the planned TS change impacted perceptions of TS stakeholder relationships and the change itself, the second objective is more focused on whether the 'sharedness' lens provides a useful perspective to understand how those perceptions may be impacted by the planned TS change. Even though previous research has shown that 'sharedness' may impact how change unfolds (e.g. impact on decision-making processes) (Tindale \& Kameda, 2000), we could find no evidence to suggest that anyone has investigated 'sharedness' in throughout a planned TS change. 


\section{CHAPTER 4: RESEARCH DESIGN}

A longitudinal, multi-method, exploratory, single-case case study design was selected to test the theoretical framework outlined in Figure 6 and address the research objectives and questions outlined in the previous section. Case studies are rich empirical descriptions of particular instances of real-life situations that typically draw on data from a variety of sources (Eisenhardt \& Graebner, 2007) and examine a phenomenon within its context (Yin, 2003). The role of context is a defining feature of case studies, as other approaches such as experiments and surveys attempt to examine variables separate from context (Yin, 2003). This makes this methodological approach particularly relevant when an in-depth understanding of the phenomenon is required and where the relationship between the context and the phenomenon is unclear (Patton, 2002; Yin, 2009).

The lack of theoretical and empirical development that exists within the TS change literature would suggest that an investigation of how a planned TS change impacts perceptions of TS stakeholder relationships would benefit from a methodology that can capture context. Although the stakeholder literature suggests that stakeholders influence how organizations behave, theory has not been developed exploring either how TS stakeholder relationships may impact change over time or how the implementation of a planned TS change may impact TS stakeholder relationships over time. The change literature is limited, despite being extensive and complex, to a single organization, which limits our understanding of TS change. What the change process literature has revealed, however, is that change process models are highly dependent on the situational contexts in which they are studied. All of these factors support the use of a case study methodology in the thesis research.

The first section of this chapter describes in greater detail the concept of case studies, the strengths and weaknesses of case studies, and the roadmap that was used in this research to build 
theory from case studies. The second section of this chapter describes the case itself. The unit of analysis for this study will be identified and justified in section three. The fourth section of this chapter discusses how the data used in this study were collected and analysed. Included in this section is a description of the archival records and semi-structured interview data collection techniques. The fifth section of this chapter outlines how the researcher gained the interactional experience necessary to analyze the data. The sixth section of this chapter presents the data analysis techniques and procedures that were used to analyze and triangulate the collected data. The final section discusses the challenges that we faced when collecting data for this thesis, and the implications of those challenges in terms of theory development.

\subsection{CASE STUDY METHODOLOGY}

Case studies are a research strategy that focus on understanding the dynamics present within single settings (Eisenhardt, 1989). Within this section, we justify the use of an exploratory, single-case, multi-method case study to increase our understanding of how planned TS change impacts TS stakeholder relationships over time. More specifically, the section discusses the following characteristics of case studies: types of case studies, timing of data collection, number of cases, number of sources, and case study approaches.

\subsubsection{TYPES OF CASE STUDIES}

The case study research design can vary according to the type of contribution the researcher intends to make with their research. Case studies have been employed to serve many purposes, the most typical being descriptive, explanatory and exploratory (Post \& Andrew, 1982; Babbie, 2001). A descriptive case study provides a complete description of a phenomenon within its setting, while an explanatory case study seeks to explain specifically why an event occurs (Post \& Andrew, 1982; Yin, 2003). Conversely, exploratory studies are undertaken when a researcher 
seeks to gain a better understanding of a previously unexplored phenomenon (Babbie, 2001). Given the lack of research investigating how planned TS change impacts TS stakeholder relationships over time, this research uses an exploratory case study approach.

Exploratory research is more deliberate and analytical than descriptive. Exploratory research is aimed at either developing structures suitable for future research in the area that may or may not also be case studies, or for determining the feasibility of undertaking a more extensive case study (Babbie, 2001; Yin, 2003; Myers, 2009). Exploratory research is useful for theory building (Babbie, 2001; Eisenhardt, 1989), particularly when research questions address the "how" and "why" in unexplored research areas (Edmondson \& McManus, 2007). The case study approach has also been acknowledged as a valuable research strategy for developing emergent theory or for sharpening existing theory, particularly when using data from multiple sources (Eisenhardt \& Graebner, 2007; Babbie, 2001). These theory building strengths of exploratory case study research provide further support to the decision to use an exploratory case study design in this research.

\subsubsection{TIMING OF DATA COLLECTION}

Case studies may be retrospective (i.e., conducted at a single point in time) (e.g., Weick, 1993), or real-time (i.e., conducted longitudinally over time) (e.g., Brown \& Eisenhardt, 1998). Retrospective cases rely on interviews and primary and secondary archival data that build up the number and depth of cases efficiently and so enable a researcher to cover more informants and include more cases. In contrast, real-time cases employ longitudinal data collection of interviews and observations, both of which help to mitigate retrospective sensemaking and impression management (Eisenhart \& Graebner, 2007). This research uses a longitudinal real-time approach 
in order to avoid retrospective biases. More details about data collection for this research are provided in Section 4.5.

\subsubsection{SINGLE- AND MULTIPLE-CASE CASE STUDIES}

Case study research can be built on either single or multiple cases (Eisenhardt, 1989; Myers, 2009). The multiple-case case study approach typically provides a stronger platform for building theory (Eisenhardt \& Graebner, 2007; Noor, 2008). Multiple cases allow researchers to compare cases, which provides researchers an additional means of building theory that single-case case studies do not have (Eisenhardt, 1989). The theory developed from multiple cases is also considered more robust because it is developed from a variety of empirical evidence. According to Eisenhardt and Graebner (2007), for example, multiple illustrations regarding the nature of latent constructs and their relationships, means that definitions can be more accurate and descriptions more precise. This also means that, in general, the scope of exploration and theoretical elaboration for the multiple-case case studies are broader and deeper.

The multiple-case case study approach, however, presents several challenges to researchers. First and foremost, conducing multiple-case case studies require more resources. Assuming that obtaining and maintaining relationships with sample sites over time require some level of resources from the researcher, increasing the number of sample sites would also imply an increase in the amount of resources required to conduct research. In addition to extra resource expenditure, there is an inherent complexity in selecting multiple cases that "contribute to theory development within the set of cases" (Eisenhardt \& Graebner, 2007, p. 27). This complexity comes about because each case must be chosen with care to complement other cases. This makes the process of selecting cases logically different from other types of research (Yin, 2009; Eisenhardt, 1989). Even though Eisenhardt and Graebner (2007) make a convincing argument for multiple- 
case case studies, there is added difficulty in selecting multiple cases when the phenomenon of interest (i.e. TS change) is practically difficult to research and has not been previously explored.

Single cases are the most common type of case study (Yin, 2009). They are often chosen to represent an unusual or rare circumstance, where the presence of a phenomenon is portrayed (Yin, 2009). Arguably, the most famous case study is Weick's (1993) single-case description of the Mann Gulch disaster. The uniqueness of the Mann Gulch case allowed Weick to argue in favor of the sensemaking concept. Restricting his analysis to that of a single case allowed Weick to delve into detail to depict how the phenomena influenced the situation. Even though the context dependent nature of single cases limits their generalizability, Sigglekow (2007) and others (e.g., Yin, 2009) recommend researchers use single cases, by arguing that such a strategy allows the researcher to focus their attention on how the phenomenon of interest may influence a single setting rather than how multiple settings may influence the phenomenon of interest. The complexity and uniqueness of the PMI process support the decision to use a single-case approach in this research.

\subsubsection{MULTI-METHOD CASE STUDIES}

Case studies may collect data using a single-method or a multi-method approach. The concept of triangulation suggests a multi-method approach for collecting data is preferred over a single-method approach (Patton, 2002).

Triangulation refers to the use of multiple techniques to reduce bias present in any single perspective (Myers, 2009; Yin, 2009). The benefits of triangulation include "increasing confidence in research data, creating innovative ways of understanding a phenomenon, revealing unique findings, challenging or integrating theories, and providing a clear understanding of the problem" (Thurmond, 2001, p.254). Patton (2002) identifies four types of triangulation including 
data (i.e., involves time, space and persons), investigator (i.e., involves multiple researchers in an investigation), theoretical (i.e., involves using more than one theoretical scheme in the interpretation of the phenomenon), and methodological (i.e., involves using more than one method to gather data). Yin (2009) argues methodological triangulation encourages the use of multiple collection methods aimed at substantiating or highlighting the same phenomena that are pertinent to the case study. Triangulation using both qualitative and quantitative data is recommended to provide richer and stronger evidence from which new theories can arise (Eisenhardt, 1989; Siggelkow, 2007).

This study intends to capitalize on many of the benefits of triangulation by: (1) developing a preliminary theoretical framework (Chapter 3) based on theory from three bodies of literature and (2) using a multi-method approach for conducting case studies that incorporate qualitative and quantitative data collection. More detail on qualitative and quantitative data collection methods are provided later in this chapter.

\subsubsection{CASE STUDY APPROACHES}

The role qualitative and quantitative data plays in case studies is largely determined by the approach researchers take when conducting case study research. Three approaches, or principles of reasoning, have appeared in the literature: deductive, inductive and abductive (Ong, 2012). When a case study uses deductive reasoning, the procedure is similar to an experiment: a hypothesis is formulated, and testable consequences are derived from deduction. Case studies inductively generating theory or conceptualizations are based on data from within a case. Abduction combines deductive and inductive reasoning and refers to an ability to act based on the conceptualization of a case (Ong, 2012). Given the focus of this research on building theory from facts in a case, this case study adheres to an inductive approach. 
Inductive case study research involves qualitative data collection and analysis as the primary data type that drives theory development. Qualitative data is useful for exploring and understanding the underlying relationships in the phenomena of interest (Eisenhardt, 1989b), which in this case involves planned TS change.

Inductive case study research may also utilize quantitative data to complement and support qualitative analysis (Creswell, 2009; Eisenhardt, 1989). For instance, quantitative data may be used to assess a large sample of participants with the intention of identifying key participants for further qualitative research (Creswell, 2009). Quantitative data can also corroborate findings from qualitative evidence (Eisenhardt, 1989).

This exploratory research uses a single case, multi-method, longitudinal case study design to inductively investigate how planned TS change impacts TS stakeholder relationships over time. The next section describes a roadmap that is frequently used to conduct inductive case study research.

\subsubsection{INDUCTIVE CASE STUDY ROADMAP}

Roadmaps are useful guides that provide instruction and facilitate how to perform a task. In relation to conducting management research, there have been many roadmaps offered to researchers that aid in conducting defendable, relevant research (e.g., Gilmartin \& D'Aunno, 2007; Jaworski \& Kohli, 1996; Mennecke et al., 2007; Papazoglou, Traverso, Dustdar \& Leymann, 2008; Vermesan et al., 2011).

Roadmaps can be general and act as a guide of what to research, such as the case with Gilmartin and D'Aunno (2007) who reviewed the leadership literature and produced a roadmap describing what leadership researchers should do to advance leadership research. Conversely, roadmaps can be detailed and outline appropriate methodologies for conducting research, such as 
the case with Jaworski and Kohli's (1996) roadmap that specifies the appropriateness of certain methodological approaches when conducting market orientation research. In the case of this thesis, this research utilizes a roadmap that illustrates how to conduct inductive case study research that was developed by Eisenhardt (1989).

Eisenhardt (1989) laid out well-defined steps of what activities are required to carry out case studies for theory development. Her roadmap is still frequently referenced, despite being over twenty years old, and used today to conduct case study research (e.g., Bingham \& Eisenhardt, 2006; Graebner, 2004; Elsbach \& Kramer, 2003). For example, Greenwood and Suddaby (2006) used Eisenhardt's roadmap to conduct case study research examining how a known instance of institutional change at the center of a field occurred (i.e., promotion of change by elite firms within the accounting profession). They justified their approach in terms of extending institutional theory and the ability of qualitative data to explicate the complex social processes involved. Similarly, this research extends TS, change, and stakeholder theories by following Eisenhardt's (1989) steps and using qualitative data to delve into the phenomena of planned TS change.

Adapted from the work of Eisenhardt (1989), Table 3 presents the process steps that are involved in this research. Key steps and activities are included in this table, as well as a column corresponding to sections of the thesis where details on how these steps/activities are to be employed in this research. The remainder of this chapter discusses the major steps involved in conducting theory-building case study research, as shown chronologically in Table 3. 


\begin{tabular}{|c|c|c|}
\hline Step & Activity & Thesis Section \\
\hline \multirow[t]{2}{*}{ Getting Started } & Definition of research question & $\begin{array}{l}3.0 \text { - } 3.2 \text { Orienting } \\
\text { Framework }\end{array}$ \\
\hline & Possibility of a priori constructs & \\
\hline \multirow{3}{*}{ Selecting Cases } & & 4.2 Case \\
\hline & Theoretical sampling & 4.3 Unit of Analysis \\
\hline & & 4.4 Sampling \\
\hline \multirow{2}{*}{$\begin{array}{l}\text { Crafting Instruments } \\
\text { and Protocols }\end{array}$} & Multiple data collection methods & 4.5 Data Collection \\
\hline & Qualitative and quantitative data combined & \\
\hline Entering the Field & Overlap data collection and analysis & $\begin{array}{l}\text { 4.5.1 Longitudinal } \\
\text { Data Collection }\end{array}$ \\
\hline \multirow[b]{2}{*}{ Analyzing Data } & Within-case analysis & 4.6 Data Analysis \\
\hline & $\begin{array}{l}\text { Cross-case pattern search using divergent } \\
\text { techniques }\end{array}$ & \\
\hline \multirow{3}{*}{ Shaping Hypotheses } & $\begin{array}{l}\text { Iterative tabulation of evidence for each } \\
\text { construct }\end{array}$ & 4.6 Data Analysis \\
\hline & Replicate logic across cases & \\
\hline & $\begin{array}{l}\text { Search evidence for "why" behind } \\
\text { relationships }\end{array}$ & \\
\hline Enfolding Literature & $\begin{array}{l}\text { Comparison with conflicting literature } \\
\text { Comparison with similar literature }\end{array}$ & 4.6 Data Analysis \\
\hline Reaching Closure & Theoretical saturation when possible & 4.6 Data Analysis \\
\hline
\end{tabular}

\subsection{CASE}

The case study involves a change to a process that occurs where police interact with persons with mental illness and bring them involuntarily to the hospital which is participating in this research. Mental illness is a clinically diagnosable disorder. Extensive examination from a trained psychiatrist is typically required to determine whether a person has a mental illness, what type of mental illness they may have, and the severity of the mental illness. This research uses the term persons with mental illness (PMIs) to refer to individuals whom the public or the police suspect may have a mental illness because their behavior endangers themselves or others. PMIs may or may not have a psychiatrically diagnosed mental illness. Within this study, we classify individuals 
perceived to have a mental illness as PMIs, regardless of their actual diagnosed disorder, because it is these perceptions that trigger the actions under the Ontario Mental Health Act (OMHA).

The OMHA is an Ontario law that governs the administration of mental health care. The main purpose of the law is to regulate the involuntary admission of people into a psychiatric hospital. There are three common ways a person may be involuntarily admitted to a psychiatric facility in Ontario for a 72-hour observation period: (1) the police bring a PMI to a doctor; (2) an individual lays information before a Justice of the Peace, who may decide to order a psychiatric assessment; or (3) a doctor orders a psychiatric assessment. This case study only examines situations where police interact with PMIs and bring them involuntarily to the hospital participating in this research.

The research "site" for this multi-method case study is a TS consisting of three organizations: a police force, a hospital, and a social services agency. This TS is mandated to manage the PMI process in the area in which the hospital is located. The PMI process refers to the set of steps that the three organizations take to address the needs of PMIs who have been brought to the attention of the police because they have endangered themselves or others. None of the three organizations involved in the PMI process can complete the process on their own. Instead, each organization relies on the unique skillsets of their two partner organizations for task completion. For example, police manage the initial crises and escort the PMI to the hospital; the hospital psychiatrically assesses and treats the PMI and lets the police know when they can leave the hospital (Canadian law requires the police to stay with the PMI until they are either admitted to the hospital or discharged); and the social service agency contributes ongoing care to the PMI back in the community to minimize the potential for future crises. 
Within the context of the conceptual framework described in Chapter 3, the police, hospital and social service agency can be conceptualized as three TS stakeholders belonging to the same TS. One of the goals of this TS, as reflected in the case, is to provide a service to the PMIs in the area of the hospital that require mental health support. As discussed in the following three sections, each of these three TS stakeholders is likely to have different perspectives on the PMI process.

\subsubsection{POLICE}

The police organization participating in this case study has over two thousand sworn $^{2}$ and one thousand non-sworn members. It also has a fleet with over 500 vehicles. The police organization has four different units: traffic enforcement, investigation, special (i.e., tactical response unit) and community support. The community support unit responds to calls for service involving PMIs.

Police officers respond to calls for service based on a priority system. The system ranges from 1 to 5 , where 1 is prioritized most urgent and typically involves immediate and emergent threats of endangerment, and 5 is prioritized less urgent and typically involves less immediate and non-emergent situations. Calls for service involving PMIs are generally prioritized 1, as they require the most immediate attention to keep the community safe.

In line with a general police mandate to maintain the community safe, the role of the police in the PMI process is to provide safety and security to the PMI and nearby bystanders. Typically, the police receive a call for service involving a PMI and need to respond immediately to manage the crisis. Section 17 of the Mental Health Act (2010) states:

"Where a police officer has reasonable and probable grounds to believe that a person is acting or has acted in a disorderly manner and has reasonable cause to believe that the

\footnotetext{
${ }^{2}$ Police officers may be sworn to an oath. This gives them a range of duties and powers including use of force options, the power to arrest and detain and use of a gun.
} 
person, (a) has threatened or attempted or is threatening or attempting to cause bodily harm to himself or herself; (b) has behaved or is behaving violently towards another person or has caused or is causing another person to fear bodily harm from him or her; or (c) has shown or is showing a lack of competence to care for himself or herself; and in addition the police officer is of the opinion that the person is apparently suffering from mental disorder of a nature... the police officer may take the person in custody to an appropriate place for examination by a physician."

When at the hospital, the police officer has to wait with the PMI until a doctor becomes available. Section 33 of the Mental Health Act (2010) states: "A police officer or other person who takes a person in custody to a psychiatric facility shall remain at the facility and retain custody of the person until the facility takes custody of him or her in the prescribed manner."

Once the doctor assesses the PMI, the police transfer the custody of the PMI to the hospital and the police are free to leave.

The role of the police in the PMI process depends on the actions of the hospital and the social service agency. The police are dependent on the hospital, as they have to wait at the hospital until a doctor psychiatrically assesses the PMI. This waiting period results in a loss of resources (man and vehicle hours) for the police. As police do not have the skillset to assess PMIs psychiatrically, they are dependent on doctors within the hospital to evaluate the PMI. The police are also reliant on the social service agency to reduce the need for calls for service with respect to PMIs in the community. Typically, the police have to respond to crises when the social service agency is not able to provide the ongoing care for the PMI that is required to maintain their mental health. In situations where the mental health of a PMI deteriorates over time, and the social service 
organization is not able to increase the care provided to that individual, there is a higher potential for an event to trigger crises for the PMI.

\subsubsection{HOSPITAL}

The hospital participating in this study is managed by an external health system that provides programs and services for the surrounding residents. The health system has more than 900 physicians, 4,300 staff, and 1,000 volunteers. The hospital itself holds more than 600 beds. The hospital provides a broad range of services to the community: cancer care, cardiac care, critical care, diabetes treatment and care, diagnostic imaging, dialysis, general and internal medicine, mental health and addictions, surgery, women's and children's healthcare services and the emergency department. The TS put in place to manage the PMI process narrows our focus to the hospital's emergency department (ED).

This hospital has a very busy ED with over 500 visitors a day. The cumulative length of time an individual or patient waits to see a doctor or specialist (i.e., wait time) as part of entering through the ED is determined by three factors: (1) the number of patients more seriously ill than the focal patient (i.e. the PMI), including those brought in by ambulance; (2) the number of tests that are required (e.g., blood, X-ray, CT scan); and (3) whether the patient needs a consultation with a specialist ${ }^{3}$.

The seriousness of all patients brought in the ED is determined by the Canadian Triage and Acuity Scale (CTAS). CTAS is a national guideline produced by the Canadian Association of Emergency Physicians and prioritizes patients in a fair manner. For example, let's look at two patients: one with a small, stable cut on their leg and another who is bleeding from their head. After comparing each patient's CTAS, the doctor would examine the patient with a head injury

\footnotetext{
${ }^{3}$ Retrieved from hospital website.
} 
before examining the patient with a leg injury, even if the patient with a leg injury arrived first. According to CTAS, the patient who is most unstable at the time should be assessed and treated first.

It should be noted that the CTAS states mental health patients "present a unique challenge for triage" (Warren et al., 2008, p. 229). While the CTAS also references several metrics that may be used to assess patients with mental illness (e.g., Lewis, 2002; Ayliffe et al., 2005), there is only a vague understanding of how to prioritize physical injuries and mental health cases in relation to each other (Warren et al., 2008). This contributes to the confusion of how to prioritize and manage PMIs entering the ED, including those PMIs brought in by police.

As per the hospital's general mandate to keep the community healthy, the role of the hospital, and more specifically the ED, in the PMI process is to assess and treat the PMI professionally and take custody of the PMI from the police. Typically, a PMI (escorted by a police officer) entering the ED is immediately triaged by a nurse and assigned a CTAS level. The PMI and police then wait to be called by the doctor. The length of wait is determined by the number and severity of other patients in the ED who have more severe cases (i.e., higher CTAS levels). Once the ED doctor assesses the PMI, the ED doctor decides whether the PMI is free to leave or needs to be detained longer for an assessment by a psychiatrist. Under Section 15 of the Mental Health Act (2010):

"Where a physician examines a person and has reasonable cause to believe that the person, (a) has threatened or attempted or is threatening or attempting to cause bodily harm to himself or herself; (b) has behaved or is behaving violently towards another person... the physician may make application in the prescribed form for a psychiatric assessment of the 
person... detain[ing] the person who is the subject of the application in a psychiatric facility

and to restrain, observe, and examine him or her in the facility for not more than 72 hours."

In the case the PMI is detained for a psychiatric assessment, a security guard from the hospital relieves the police officer of their duty and the police are allowed to leave. The PMI is then assessed and treated by the psychiatrist within 72 hours. When the psychiatrist feels the PMI is no longer at risk of endangering themselves or others, the psychiatrist discharges the PMI and the PMI is allowed to leave the hospital.

Within the PMI process, the hospital is dependent on police and social service organizations. The hospital is required to assess all PMIs brought into the ED by the police even if the PMI does not require medical attention. The more PMIs brought into the hospital by the police, the more the hospital needs to consume their own resources and assess PMIs. The hospital is dependent on the social service in that if the social service cannot provide the ongoing care that is required of the PMI, then the PMI may experience a crisis and be brought by the police to the hospital.

\subsubsection{SOCIAL SERVICE AGENCY}

For over 50 years, the social service agency participating in this case study has been a pioneer in providing services for PMIs and educating Canadians about mental health issues. As a leading, community-based mental health organization, the social service agency can be an innovative partner in proactively strengthening individual support to PMIs, and developing a responsive mental health system. In addition to providing a variety of mental health services, the social service agency is committed to community development, mental health awareness, and addressing the stigma surrounding mental illness. 
The role of the social service agency in the PMI process is to provide ongoing care and support (i.e., outside the hospital) to PMIs who need help managing their mental illness. In theory, the more care provided by this agency, the less likely the PMI is to have a crisis that requires intervention by the police and, by extrapolation, the hospital. The social service agency is dependent on the police and the hospital in that the treatment and care provided by the police and hospital to the PMI "in crisis" may influence the amount of resources the social service agency needs to spend on the PMI to manage their mental health when they return to the community.

\subsubsection{ENVIRONMENTAL SHIFTS IMPACTING THE PMI PROCESS}

The PMI process has recently been impacted by a series of major changes in the external environment. The closure of mental health hospitals (e.g., Mulvale, Abelson \& Goering, 2007) and the reduction of social service funding (e.g., Aronson \& Sammon, 2000) has increased the number of PMIs that these three organizations have to deal with in a given year and increased the financial and reputational costs of managing the PMI process for all three organizations. These changes to the external environment have, however, differentially impacted the three organizations.

The external shifts negatively impact police in two ways. First, police are consuming more resources "on the road", as they respond to a greater number of calls for service involving PMIs (and potential PMIs) than in the past (Callender, Chandrasekera, Lillie \& McGurk, 2013). Second,

police are spending considerably more time at the hospital with the PMI waiting for a doctor to become available. As police resources are strained responding to the increase in PMIs, the hospital resources are also strained. Unfortunately, as hospital resources become more strained, the hospital is less capable of responding to PMIs and forces police officers to wait longer. During 
this time, the police are not available to handle other calls in the community (i.e., decline in service).

The external shifts negatively impact the hospital in that while the ED is seeing more visits by PMIs (i.e., greater resource consumption), the hospital has not received additional funding to offset costs associated with these increases. As a result, all patients (PMIs and other visitors) are facing longer wait times. Whereas police are concerned with increased wait times for PMIs, the hospital is concerned with increased wait times for all patients as this reflects a reduction in service and may negatively impact hospital funding. ED funding in the Canadian province where the study unfolds are tied to wait times and hospitals with longer wait times may be censored by the province and see their funding cut. If the hospital is unable to meet provincially set standards for hospital wait times (based on CTAS level), the hospital would see a reduction in ED funding.

The external shifts also negatively impact the social service agency by forcing them to play a more active role in the provision of mental health care to PMIs in the community without increased funding to offset costs. These shifts see social service employees increasing the number of PMIs in their portfolio (i.e., increased case workload). This strains the social service's resources, as more PMIs are allocated to each social service employee, and reduces the service provided to PMIs, which, in turn, increases the likelihood that these individuals will experience a mental health crisis as care becomes more reactive than proactive. The reduction in public resources means that the social service agency needs to play a larger role mediating the linkage between the community and the hospital.

\subsubsection{SITUATION LEADING UP TO THE PLANNED TS CHANGE}


To address the negative impact of the changes to the external environment on the police, hospital and social service agency, the Deputy Chief of the police service, the Executive Director of Emergency Services at the hospital, and the Chief Operating Officer of the social service agency participating in this study all agreed in the Summer of 2013 to participate in a case study research project in collaboration with the researcher's supervisor and the researcher. A steering committee was created in the Fall of 2013 to guide the focus and overall execution of the study. The steering committee has representation from senior management within the police, hospital, and social service agency participating in the study.

The goal of the steering committee was to determine the best way to change how the police, hospital and social service agency manage PMIs who are involuntarily brought to the hospital by the police. All three organizations committed to changing the PMI process after an analysis of the current PMI process was completed.

This TS (i.e., police, hospital and social service agency) provided an ideal research site for the study of planned TS change for three reasons. First, this research opportunity presented a TS that was expected to undergo a change. The TS was thus highly likely to provide the opportunity to observe the phenomenon of interest for this case study (i.e., planned TS change), a factor that is important when selecting cases for case study research (Yin, 2009). Focusing the research on a particular setting also minimizes the risk that sources of extraneous variation will conflate the findings (Eisenhardt, 1989). Second, the engagement and willingness of the organizations to participate in the study facilitate the use of the qualitative techniques that are recommended for process based change research (Van de Ven \& Poole, 2005). Third, the researchers' long-term involvement with these organizations meant that the longitudinal data necessary for addressing the research objectives was accessible. 


\subsection{UNIT OF ANALYSIS}

After defining the research question(s), the next key component of case study design is to identify the unit of analysis (Yin, 2009). The unit of analysis refers to the entity, object, or event under study (Myers, 2009). According to Singleton and Straits (2010), the unit of analysis "is simply what or who is to be described or analyzed" (p.81). Yin (2003) suggests the identification of the unit of analysis is closely related to the objectives of the research and the way in which the research questions have been defined. While research studies in the management area have traditionally only had a single unit of analysis, Scandura and Williams (2000) review methodological practices within the management literature to argue that there may be value in conducting research with multiple units of analysis. More specifically, they contend that larger research studies may employ triangulation from a level of analysis perspective to "improve internal and external validity as the combination of separate research strategies in one study helps to counter the trade-offs inherent in others" (Scandura \& Williams, 2000, p.1252). Accordingly, this research was designed to include two different units of analysis: the stakeholder and the individual. The rationale behind this decision is given below.

First, the research objectives, the research questions, and the conceptual framework for this research lead us to identify the TS stakeholder as one of the two units of analysis. Choosing the TS stakeholder as the unit of analysis means that this research involves three units (i.e., police, hospital, and social service agency) who are linked to each other within the PMI process. Accordingly, this choice of unit of analysis supports our primary objective of understanding how planned TS change impacts perceptions of TS stakeholder relationships over time by exploring three sets of TS stakeholder perceptions within the framework of a single case. 
Second, as TS stakeholder level phenomena are usually impacted by phenomena at the individual level (Snijders, 2011), this thesis also undertakes a limited set of analyses where the individual is the primary unit of analysis. More specifically, our initial analysis of the data suggested that individual sensemaking impacted perceptions of planned TS change over time. To enrich our understanding of how the planned TS change impacts TS stakeholders over time, we undertook a follow-up analysis using a sensemaking lens where the analysis was focused on the responses given by our respondents (see Section 5.4).

\subsection{SAMPLING STRATEGY}

A very important part of theory-building case studies is case selection (Myers, 2009). Eisenhardt (1989) suggests that case study researchers use purposeful or theoretical sampling practices when selecting cases for inclusion in their study as a means to better focus their attention on theoretically useful cases. In this research, we seek to understand how planned TS change impacts TS stakeholder relationships over time by examining TS stakeholders' employees' perceptions of each other and the change itself. The primary criteria for inclusion of a TS stakeholder into this study is that the TS stakeholder: (1) has a member of their organization participate on the steering committee that is dedicated to improving the PMI process, and (2) participates in the TS before the intervention (i.e., Time 1) as well as after the intervention (i.e., Time 2).

The first criterion ensures that the TS stakeholder is directly involved in changing the PMI process. Although other groups, such as the families of patients, may also influence TS change, they are not included in this case study, as they have not participated in the process of planning and implementing PMI process change. This criterion touches on the idea of intensity sampling. Intensity sampling logic seeks the selection of cases that exhibit the dimension of interest intensely, 
so as to maximize the availability of rich information on the phenomenon (Patton, 2002). In this case, this meant that our study focused on those TS stakeholders who are the most connected to the planned TS change process.

The second criterion ensures the researcher can assess the impact of time on the participants' responses. In other words, it allows the researcher to compare the planned TS changes to TS stakeholders' perceptions of what actually changed within the TS. It is not possible to test the preliminary orienting framework if the sampling strategy does not meet this condition.

Case studies using qualitative methods frequently require researchers to determine sample size based on saturation, not population representativeness (Charmaz, 2006; Morse et al., 2009). Saturation refers to the point at which "the collection of new data does not shed any further light on the issue under investigation" (Mason, 2010, p.1). As saturation depends on a number of factors, such as familiarity with the topic and experience in the field, the identification and demonstration of the saturation point is very difficult (Mason, 2010). To help newer researchers identify saturation, some guidelines have been provided regarding approximate sample sizes in order to reach saturation in theory building studies using interviews. Some recommended sample sizes for qualitative interview studies are 20-30 (Creswell, 1998), 30-50 (Morse, 1994) and 25 (Charmaz, 2006).

Based on the above recommendations, the target sample size for the longitudinal interview data set of this study is 25 respondents per TS stakeholder, totaling 75 respondents.

\subsection{DATA COLLECTION}

The objective of this section is to provide the reader with an understanding of the data collection strategy for this inductive case study research. Inductive case studies use cases as the 
basis from which theory emerges inductively (Eisenhardt \& Graebner, 2007). Collecting data in research that has emergent theory can present major challenges associated with planning (Goulding, 2002). These data collection techniques rely heavily on the researcher's ability to alternate between complete immersion in the data and separation from the data until categories, properties, and relationships emerge (Goulding, 2002). Predicting exactly what types of data will ultimately contribute to the emergence of key categories and theory is extremely challenging (Goulding, 2002; Ng \& Hase, 2008). We used two strategies during the data collection to overcome this challenge. First, the researcher utilized data from multiple time periods. Second, the researcher utilized multiple data collection methods. These two strategies are described below.

Longitudinal research concerns the collection and analysis of data over time and is important in process studies of change. As Van de Ven and Huber (1990) noted, the question of 'how' change emerges, develops, continues, and terminates over time remains largely unanswered because most change researchers engage in cross-sectional research. Armenakis and Bedian (1999, p. 311) state that future research into the temporal sequence of events that unfold as change occurs would provide a deeper understanding of the dynamics of change and permit the testing of current and new theories addressing 'how' organizations (or in our case the TS and TS stakeholders) change.

Longitudinal research can be defined as research in which: (1) data are collected for each item or variable for two or more distinct periods; (2) the subjects or cases analysed are the same, or at least comparable, from one period to the next; and (3) the analysis involves some comparison of data between or among periods (Menard, 1991, p.4). Ruspini (2000) outlines two different types of data that may be regarded as longitudinal: repeated cross-sectional studies and prospective designs. First, repeated cross-sectional studies involve cross-sectional data recorded in a 
succession of surveys or interviews at two or more points in time, with a new sample on each occasion (ibid). Where cross-sectional data are repeated over time with a high level of consistency between questions, it is possible to incorporate a time trend into the analysis. Second, prospective designs involve interviewing the same individuals repeatedly across time (ibid). One variation of this design is representative panels, which involve a random sample of respondents who are repeatedly interviewed at fixed intervals (Buck et al., 1994). As this type of longitudinal research allows the researcher to detect and establish the nature of individual change, they are well-suited for the analysis of both social change and dynamic behavior (Ruspini, 2000).

Prior to any planned changes to the TS, benchmark (Time 1) data were collected from the police database, as well as 75 respondents within the TS. These data provide us with contextual information on the TS, as well as information on how the respondents in our TS view two constructs of interest to this study: Perceptions of TS Stakeholder Relationships and Perceptions of Desired TS Changes. Time 1 data consist of archival data and semi-structured interviews.

Time 2 data, which were used to provide contextual information on the TS post-change and to identify TS stakeholder perceptions of what has changed in the PMI process over time and perceptions of how TS stakeholder relationships have changed over time, were collected approximately six months after the planned changes were implemented. The Time 2 data were collected from police and hospital in the form of archival records and semi-structured interviews. Data were collected from the social service agency in the form of semi-structured interviews. The two data collection methods are explained and their use in this study justified in the next three sections.

\subsubsection{ARCHIVAL RECORDS}


Archival data is important when studying change because it provides benchmark (or tombstone) data on how the system initially operates, and facilitates comparison to data describing how the system operates after changes have been made (Womack \& Jones, 1996). Archival research involves seeking out and extracting evidence from original archival records relating to constructs of interest (Singleton \& Straits, 2010). Archival research allows researchers to avoid response bias and investigate a large amount of data efficiently (Singleton \& Straits, 2010). Response bias refers to a tendency of a respondent to answer in a certain biased direction (such as in the direction of social desirability) as a function of the content or form of survey questions (Singleton \& Straits, 2010). Archival research techniques have been used in many studies to examine specific populations (e.g., Logan, Stults \& Farley, 2004), and often add a critical perspective to understanding case studies of lean processes (e.g., Bhasin \& Burcher, 2006). The strengths of archival research suggest that this data collection technique may be appropriate to complement and corroborate the qualitative findings within a case study that involves perceptions from multiple different TS stakeholders at two points in time.

There were two sets of archival records that were collected and analyzed in this case study. More specifically, the police provided an Excel spreadsheet to the researcher that identified all occurrences (they refer to as "calls for service") where police were called to service for a mental health related issue in 2013 and 2014. Similarly, the hospital provided an Excel spreadsheet to the researcher that identified all occurrences (they refer to as "visits") where patients suspected to have a mental illness visited the ED in $2014^{4}$. This archival data allowed us to calculate a variety of quantitative indicators (e.g. wait times) pre and post the introduction of the planned TS change,

\footnotetext{
${ }^{4}$ Initial investigation of the hospital data revealed that, prior to the start of this study, the hospital was unable to identify patients brought in by police. They immediately changed how they tracked patients and began tracking mental health patients brought in by police in December 2013.
} 
and thus provide us with objective information on the extent to which the planned TS change actually led to changes in the PMI process. These data could be then compared to qualitative data on respondents' perceptions of change. More details on the type of archival data collected (Section 5.1) and the analysis of the indicators (Section 5.2) are provided in the following chapter.

\subsubsection{SEMI-STRUCTURED INTERVIEWS}

One-on-one, semi-structured interviews are used as the primary source of data for this case study. One-on-one interviews are valuable instruments for data collection in inductive case studies because they are "open-ended yet direct, shaped yet emergent, and paced yet unrestricted" (Charmaz, 2006, p. 28). In a semi-structured interview, the interviewer uses an interview guide with specific questions that are organized by topics but are not necessarily asked in a specified order (Bailey, 2007). Interviews are usually used in case study research and allow the researcher to gather rich data from people in various roles and situations (Myers, 2009).

There were two sets of interviews for this case study: Time 1 interviews conducted before the introduction of the planned TS change and Time 2 interviews conducted 6-months after the implementation of the change. The complete interview script for Time 1 and Time 2 interviews can be found in Appendix 1 and 2. Table 4 summarizes the constructs of the preliminary orienting framework, the research questions associated with these constructs, and the interview questions used to collect the data needed to address the objective and answer our research questions. The next four sections provide details on each of the open-ended questions listed in Table 4. 


\begin{tabular}{|c|c|c|}
\hline Model & Research Questions & Interview Questions \\
\hline $\begin{array}{l}\text { Perceptions of } T S \\
\text { Stakeholder } \\
\text { Relationships at } \\
\text { Time } 1 \text { and Time } 2\end{array}$ & $\begin{array}{l}\text { How do TS stakeholders' perceptions of (a) } \\
\text { power, (b) legitimacy, and (c) urgency } \\
\text { within the TS before the implementation of } \\
\text { planned change compare to their perceptions } \\
\text { of (a) power, (b) legitimacy, and (c) urgency } \\
\text { six months after the implementation of the } \\
\text { planned TS change? }\end{array}$ & $\begin{array}{l}\text { Time } 1 \text { and Time } 2 \text { : } \\
\text { How is power displayed in the PMI process? } \\
\text { How do the police display power in the PMI } \\
\text { process? } \\
\text { How does the hospital display power in the } \\
\text { PMI process? } \\
\text { How does the social service agency display } \\
\text { power in the PMI process? } \\
\text { How is legitimacy displayed in the PMI } \\
\text { process? } \\
\text { What makes it socially acceptable or okay } \\
\text { for an organization to be involved in the } \\
\text { PMI process? } \\
\text { Why is each stakeholder involved in the } \\
\text { PMI process? } \\
\text { Why does an organization participate in the } \\
\text { PMI process to begin with? } \\
\text { What actions in the PMI process would you } \\
\text { consider urgent? } \\
\text { Why do you say this? }\end{array}$ \\
\hline $\begin{array}{l}\text { Perceptions of } \\
\text { What Has } \\
\text { Changed In the TS } \\
\text { at Time } 2\end{array}$ & $\begin{array}{l}\text { How do TS stakeholders' perceptions of } \\
\text { what needs to be changed with the TS } \\
\text { compare to their perceptions of what has } \\
\text { changed within the TS six months after the } \\
\text { implementation of the planned TS change? }\end{array}$ & $\begin{array}{l}\text { Time 1: } \\
\text { What parts of the PMI process need to be } \\
\text { changed? } \\
\text { Why these parts? } \\
\text { What changes would you personally make to } \\
\text { the PMI process to make it more effective? } \\
\text { Time } 2 \text { : } \\
\text { What have you observed has changed in the } \\
\text { PMI process over the past couple of } \\
\text { months? } \\
\text { Why do you say this? }\end{array}$ \\
\hline $\begin{array}{l}\text { Perceptions of } \\
\text { What Has } \\
\text { Changed In the TS } \\
\text { at Time } 2\end{array}$ & $\begin{array}{l}\text { How do TS stakeholders' perceptions of } \\
\text { what has changed in the TS six months after } \\
\text { the implementation of the planned TS } \\
\text { change compare to their perceptions of (a) } \\
\text { power, (b) legitimacy, and (c) urgency six }\end{array}$ & $\begin{array}{l}\text { Time 2: } \\
\text { How is power displayed in the PMI process? } \\
\text { How is legitimacy displayed in the PMI } \\
\text { process? } \\
\text { What actions in the PMI process would you } \\
\text { consider urgent? }\end{array}$ \\
\hline $\begin{array}{l}\text { Perceptions of TS } \\
\text { Stakeholder } \\
\text { Relationships at } \\
\text { Time } 2\end{array}$ & $\begin{array}{l}\text { months after the implementation of the } \\
\text { planned TS change? }\end{array}$ & $\begin{array}{l}\text { What have you observed has changed in the } \\
\text { PMI process over the past couple of } \\
\text { months? }\end{array}$ \\
\hline
\end{tabular}

Table 4: Mapping interview questions to Orienting Framework. 


\subsubsection{BACKGROUND INFORMATION}

This part of the interview was designed to collect pertinent background information about the respondent and their background with respect to the PMI process. It includes questions asking respondents to describe what they did for a living, what their position was (if applicable), how they interacted with PMIs (e.g., provided care as a nurse) and how frequently they interacted with PMIs. Identical information was collected in both the Time 1 and Time 2 interviews.

\subsubsection{PMI PROCESS}

This section, which was only included in the Time 1 interview script, was designed to give us an understanding of the PMI process. This information was used by the steering committee when identifying the planned TS changes. An approach typified by Dutton et al. (2001) was used to develop the interview script for this task. In their study on change, Dutton et al. (2001) asked each respondent to describe a personal attempt to bring an issue to the attention of top management. Through a partially directed interview protocol, the respondents were asked to describe the major steps in a particular process and how they interpreted them. Specific probes were used by Dutton et al. (2001) to ask additional questions about the overall context, what worked well, and what could have been done differently. Their goal was to capture, as completely as possible, an account of the process in question as it unfolded over time.

This approach fits quite well with the objectives of this thesis and was used to capture accounts of the PMI process as experienced firsthand by the respondent. Part II of the interview started with the following preamble:

"The majority of police organizations and hospitals in Ontario have been affected by the closure of mental health hospitals and the subsequent increase in the number of calls to the police involving persons with mental illness. Police and 
hospitals have been working together to manage this situation. Currently there are a set of steps that are undertaken when police and health care services are asked to manage a "call for service" dealing with a person with mental illness - we refer to this set of steps as the PMI process. The next set of questions were designed to give us a greater understanding of how this process works from your perspective.

I would like you to walk me through the steps of the PMI process as you have experienced it (e.g. from your point of view)."

To receive a more complete description of the respondent's perspective of the PMI process, the researcher used several prompts. These prompts, such as asking "why", "what did you do next" or "how long did that take", encouraged the respondent to discuss the PMI process in detail. Although this format of prompting further discussion of the PMI process increased the length of time it took to conduct each interview, the responses from this section of the interview provided the researchers with background information on the PMI process (e.g., steps in the PMI process) to help them interpret responses to questions from other sections of the interview.

\subsubsection{TS STAKEHOLDER RELATIONSHIPS}

This section appeared in both Time 1 and Time 2 interview scripts. The questions in this section follow an approach used by Parent and Deephouse (2007) and Mitchell et al. (1997) in their work on stakeholder analysis. In their study on the committee responsible for organizing the Pan Am games, Parent and Deephouse (2007) asked respondents to first identify who they felt were the relevant stakeholders in their research (i.e., stakeholders to the organizers of the Pan Am games), and then to discuss their relationship with the stakeholders based on Mitchell et al.'s (1997) power, legitimacy and urgency attributes. The current research used a similar approach.

We began by asking all TS stakeholders to identify stakeholders to the PMI process. This line of 
questioning helped the researchers determine whether or not they had the appropriate TS stakeholders in the steering committee and when conceptualizing the TS. We then asked a number of questions to help us understand the perceived relationships between the various stakeholders using Mitchell et al.'s (1997) stakeholder framework as a lens through which to navigate these discussions.

To explore TS stakeholder relationships, informants were asked questions about how power, legitimacy and urgency were displayed in the PMI process and who displayed these attributes. According to Mitchell et al. (1997), power is the ability of an entity to get someone to do something they do not want to do. We asked respondents at Time 1 and then again at Time 2: "How is power displayed in the PMI process?" Several additional questions, which asked "How do the police display power in the PMI process?", "How does the hospital display power in the PMI process?", and "How does the Social Service Agency display power in the PMI process?", were used as prompts to ensure respondents considered how power was displayed (or not displayed) by each of the partners in the PMI process.

Legitimacy refers to the generalized perception that the actions of a stakeholder are desirable, proper, or appropriate within some socially constructed system of norms, beliefs, and values (Suchman, 1995). Our pre-test of the interview questions determined that respondents had problems interpreting questions that included the word "legitimacy". This finding is consistent with prior research in the area that has reported that respondents often have considerable difficulty responding to interview questions that use the word 'legitimacy' (Parent \& Deephouse, 2007), as the legitimacy construct is vague and imprecise (Mitchell et al., 1997) and requires interpretation at the individual, organizational, and societal levels of analysis (Suchman, 1995). To develop the appropriate wording for our interview question associated with legitimacy, we looked at how 
others had conceptualized legitimacy. This review revealed that legitimacy: (1) refers to socially accepted or expected behaviors (Davis, 1973), (2) is a product of action (Drori \& Honig, 2013), and (3) is considered a tool for consolidating organizational reputation, either externally (Suddaby \& Greenwood, 2005) or internally (Bitektine, 2011).

Accordingly, the line of questioning we used in our Time 1 and Time 2 interview scripts to capture respondents' perceptions of TS stakeholder legitimacy involved asking questions to help us understand legitimacy using a social acceptability lens. More specifically, we asked respondents: "How is legitimacy displayed in the PMI process?" We prompted this question by asking: "What makes it socially acceptable or okay for an organization to be involved in the PMI process?"

Urgency refers to the degree to which managerial delay in attending to the claim or relationship is unacceptable to the stakeholder (Mitchell et al., 1997). In order to gain an understanding how respondents perceive urgency within the TS before planned changes were implemented, we asked respondents at Time 1 and Time 2: "What actions in the PMI process would you consider urgent?" We then used the answers from the section of the interview on the PMI process to determine which TS stakeholder was responsible for this activity.

\subsubsection{TS CHANGE MANAGEMENT}

The final section of both interview scripts sought to capture respondents' perceptions of TS change. In the Time 1 interview script, questions were asked to help us understand what types of changes each of the TS stakeholder groups would like to see within the PMI process (i.e. Desired TS Changes). More specifically, we asked respondents at Time 1: "What parts of the PMI process need to be changed?" Several additional questions, which asked "Why these parts?" and "What 
changes would you personally make to the PMI process to make it more effective?" were used to further prompt respondents.

In the Time 2 interview script, questions were asked to help us understand what respondents perceived had changed in the TS over time. We did this by asking respondents during the Time 2 interview: "What have you observed has changed in the PMI process over the past couple of months?"

\subsection{INTERACTIONAL EXPERTISE}

To study people, subject matter, and their context in meaningful ways, Collins (2004) argues that researchers need to acquire "interactional expertise", which they define as the kind of knowledge required for one to communicate about a domain without necessarily being able to practice in that domain (see Collins \& Evans, 2007). Researchers who have honed their interactional expertise know how to relate to practitioners (e.g., police officers, healthcare professionals, social workers) in ways that engage them in sharing what they know, technical content, and what is going on in the setting. This involvement provides researchers not only access to, but also an appreciation of practitioners' views, activities, and interests, which helps them better interpret what practitioners are saying. Bruns (2013) provides a nice example of developing interactional expertise in her work. She studied cancer biology lab practices for 6 months before starting her 18-month ethnographic field study of 12 cancer research projects, pointing out that direct work exposure is important for understanding how scientists apply specialized knowledge from other disciplines.

For this case study, the researcher developed interactional expertise through: (1) passively observing participants as they were involved in the PMI process, and (2) conducting focus groups. Details on each of these methods are given below. 
Participant observation refers to observing participants in their natural setting as they go about their regular business (Bailey, 2007). Three forms of participant observation provided the researcher with interactional expertise. First, the researcher observed the PMI process from the police perspective during 60 hours of 'ride alongs' in September 2013. A 'ride along' is an arrangement for a civilian (i.e. the researcher) to spend a shift in the passenger seat of a police vehicle, observing the work day of an officer. Second, the researcher observed the PMI process from the hospital perspective during 10 hours of emergency department shadowing in September 2013. Emergency department shadowing is when an emergency department nurse allows an individual (i.e., the researcher) to follow and observe as they perform their daily activities. Third, the researcher observed the group decision-making process that took place within the steering committee after Time 1 data was collected and before changes were implemented. Observing the group decision-making process allowed the researcher to get a better understanding of what issues within the PMI process were more important to the managers from each TS stakeholder. Throughout all three forms of participant observation, the researcher passively observed and took notes, while the practitioners went about their daily routine.

Focus groups are semi-structured discussions among a small group of participants led by a skilled interviewer (Myers, 2009). Focus groups are often used to learn how people think about a topic (Fowler, 1995; Krueger \& Casey, 2000). Two focus groups were conducted in September 2013 that involved PMIs and PMI family members. Both focus groups were three hours in length and facilitated by the researcher and experienced staff from the social service agency. Two questions were asked during these focus groups: (1) "Think back to the times you were brought to the hospital by police and didn't want to go with them. Can you give me a couple words to describe what you remember most about these experiences?", and (2) "Can you give me a couple words to 
describe how you felt during this experience?" These focus groups provided the researcher with interactional expertise with respect to how the PMI viewed the process.

\subsection{DATA ANALYSIS}

According to Eisenhardt (1989) and her steps for building theory from case study research (see Table 3), researchers entering the field and collecting data should overlap data collection and analysis. Yin (2009) argues that data analysis be driven by the unique combination of qualitative and quantitative data. Although both qualitative and quantitative data were collected for this study, qualitative data were the primary type of data used to address the research objectives for this study. Both Eisenhardt (1989) and Yin (2009) have acknowledged Miles and Huberman (1994) as the formulator of a comprehensive methodology for the analysis of case based qualitative data. Accordingly, Miles and Huberman's (1994) approach was used for this case study.

Miles and Huberman (1994) recommend that case studies utilize a systematic way to store collected data. Yin (2009) also recommends that a suitable software application be used to support qualitative data analysis. QSR-Nvivo Version 10, a software package that facilitates qualitative data analysis, was used in this research to sort and code the collected data.

In preparation for the data analysis, all of the recorded interviews were transcribed, saved as Microsoft Word documents, and then imported into Nvivo to form the source documents for the data analysis. We used Nvivo's classification function to note the respondent's membership within a TS stakeholder unit (i.e., police, hospital, social service). Classifying respondents by TS stakeholder facilitates the cross-case analysis that took place later in the data analysis procedure.

Once the data has been imported and appropriately classified, the data can be analyzed, triangulated, and interpreted (Eisenhardt, 1989; Yin, 2009). The following sections describe how data were analyzed (Section 4.7.1), interpreted (Section 4.7.2), and triangulated (Section 4.7.3). 


\subsubsection{CASE BASED QUALITATIVE DATA ANALYSIS}

Miles and Huberman's (1994, p.56) first stage in data analysis is undertaken to reduce the large volume of qualitative data collected during the interview process. This is done by having the researcher develop codes to serve as "retrieval and organizing devices" that subsequently allow the researcher to manipulate the data more easily. Miles and Huberman (1984) describe codes as abbreviations, or tags, for assigning concise meanings to a segment of descriptive data. This iterative process produces a structured representation of the coded data, commonly referred to as a coding scheme. Assigning codes in this way facilitates the process of identifying and aggregating all data segments relevant to a particular theme, construct, or research question. Moreover, codes can be counted, organized, aggregated and displayed to support theoretical inferences (Johnson et al., 2007). The following two subsections describe how the coding scheme was created (Section 4.7.1.1) and displayed (Section 4.7.1.2).

\subsubsection{DEVELOPING THE CODING SCHEME}

The development of the coding scheme for Time 1 data consists of five steps. The first step involved grouping the data by TS stakeholder and reading through all of the interview transcripts associated with a single TS stakeholder. The second step involved identifying first-order codes within the data associated with a single TS stakeholder. This step follows the advice of Eisenhardt (1989) who recommends that the researcher conducts within-case analysis prior to cross-case analysis. These first-order codes are assigned to segments of text and are typically descriptive (Yin, 2009). After the first TS stakeholder group's transcripts were coded, the researcher repeated this step for the other two groups.

As the analysis and coding continues on a within-TS stakeholder basis, the researcher becomes more knowledgeable about the dynamics of each TS stakeholder, and may also note 
patterns emerging across TS stakeholders. The third step in the coding scheme process involves grouping first-order codes into second-order categories. These second-order categories are assigned to highlight cross-case patterns and to add elements of interpretation to the coding scheme as they are confirmed (Miles \& Huberman, 1994). If too many segments of data get the same second-order category, new first-order codes are created and the coding scheme is revised. Accordingly, the coding scheme is continually updated and refined as the researcher analyzes the interview data (Miles \& Huberman, 1994). The process continues in the fourth and final step as similar categories are gathered into aggregate theoretical dimensions, which create the constructs in the conceptual framework.

An example of the coding scheme created from the Time 1 data is presented in Table 5 to illustrate the data analysis process. This example comes from responses to the question "How is power displayed in the PMI process?" In this example, the table highlights the step by step creation of one specific branch of the coding scheme that was developed using Nvivo. Each step is represented by a column in the table. 


\begin{tabular}{|c|c|c|c|c|}
\hline Text Segment & $\begin{array}{l}\text { First-Order } \\
\text { Coding }\end{array}$ & $\begin{array}{l}\text { Second-Order } \\
\text { Categories }\end{array}$ & $\begin{array}{l}\text { Aggregate } \\
\text { Theoretical } \\
\text { Dimensions }\end{array}$ & $\begin{array}{l}\text { Construct in } \\
\text { Conceptual } \\
\text { Framework }\end{array}$ \\
\hline $\begin{array}{l}\text { "They [ED physicians] can change the } \\
\text { medication the client takes... it can } \\
\text { change the plan we need to follow." } \\
\text { (Social Service Agency Frontline) }\end{array}$ & $\begin{array}{l}\text { Doctor decides } \\
\text { PMI's treatment }\end{array}$ & \multirow{3}{*}{$\begin{array}{l}\text { Perceived Power } \\
\text { Displayed by } \\
\text { Hospital }\end{array}$} & \multirow{5}{*}{$\begin{array}{l}\text { Perceptions of } \\
\text { TS Stakeholder } \\
\text { Power }\end{array}$} & \multirow{7}{*}{$\begin{array}{l}\text { Perceptions of } \\
\text { TS } \\
\text { Stakeholder } \\
\text { Relationships }\end{array}$} \\
\hline $\begin{array}{l}\text { "It depends on the staff who are on but } \\
\text { definitely the staff are in control of } \\
\text { when the police will get to speak with } \\
\text { the physician or with their patient and } \\
\text { how long they will wait there." } \\
\text { (Hospital Frontline) }\end{array}$ & $\begin{array}{l}\text { Hospital staff } \\
\text { decide when } \\
\text { police officers } \\
\text { can leave }\end{array}$ & & & \\
\hline \multirow[t]{5}{*}{$\begin{array}{c}\text { "We have the power to keep them for } \\
72 \text { hours... We can decide whether } \\
\text { we're going to keep you or whether } \\
\text { we're going to let you go." (Hospital } \\
\text { Manager) }\end{array}$} & $\begin{array}{l}\text { Doctor decides } \\
\text { whether PMI is } \\
\text { formed }\end{array}$ & & & \\
\hline & & $\begin{array}{c}>\text { Perceived Power } \\
\text { Displayed by Social } \\
\text { Service Agency }\end{array}$ & & \\
\hline & & $\begin{array}{l}>\text { Perceived Power } \\
\text { Displayed by Police }\end{array}$ & & \\
\hline & & & $\begin{array}{c}>\text { Perceptions of } \\
\text { TS Stakeholder } \\
\text { Legitimacy }\end{array}$ & \\
\hline & & & $\begin{array}{c}>\text { Perceptions of } \\
\text { TS Stakeholder } \\
\text { Urgency }\end{array}$ & \\
\hline
\end{tabular}

Table 5: Example of developing the coding scheme

The first column of Table 5 represents the raw data and gives three examples of comments made by respondents from different TS stakeholders. Each describes details of one way that the Hospital is perceived to display power within the TS. Each of these text segments were coded into first-order codes using Nvivo. The names given to these first-order codes are displayed in the second column. This coding process exemplifies the first stage within-case analysis process described above. As the data analysis progressed, certain text segments were coded as "Doctor decides PMI's treatment", "Hospital staff decide when police officers can leave", "Doctor decides whether PMI is formed", or other first-order codes not shown in the example. 
Once first-order codes were assigned to interview data from all three TS stakeholders, the researcher began to look for similarities between first-order codes within and across TS stakeholders. For the example shown in Table 5, the researcher noted the similarities for the three first-order codes and grouped them into a second-order category called "Perceived Power Displayed by Hospital", as shown in column three. A similar process was conducted (but not shown in the example) for other second-order categories including "Perceived Power Displayed by Social Service Agency", and "Perceived Power Displayed by Police".

As the data analysis continued, the researcher noted similarities between the second-order categories and grouped these categories into the aggregate theoretical dimension of "Perceptions of TS Stakeholder Power". The aggregate theoretical dimensions for this example are shown in the fourth column. The three aggregate theoretical dimensions identified for this particular example were then linked to a construct within the conceptual framework: Perceptions of TS Stakeholder Relationships at Time 1. Table 5 (see above) and the accompanying description thus illustrate the data analysis process from raw data to a structured set of connected data for one construct of the conceptual framework.

\subsubsection{DISPLAYING CODED DATA}

The next stage of data analysis involves displaying the coded data. Eisenhardt and Graebner (2007) assert the best way to build theory from case studies is to develop theory in sections, organize the results around the theory, and make extensive use of tables and other visual devices to summarize case evidence. Miles and Huberman (1994) also contend that evidence for theoretical constructs should be presented in separate tables.

Miles and Huberman (1994) suggest there are two primary benefits of counting qualitative data codes. First, these displays of counts enable the researcher to get a clearer picture of what the 
coded data is showing by indicating the relative importance of a particular coded response. Second, counts are also used to observe patterns and highlight data that suggests leads for further investigation.

The format used to display coded data in this research was driven by the research questions and conceptual framework shown in Figure 6, and by the coding scheme as articulated in the previous section. Data displays allow the researcher to develop initial propositions to explain how planned change unfolds in a TS over time by observing the relationships between possible variables (Miles \& Huberman, 1994). Accordingly, the data displays in this research are used, not only to present the results, but also to continue the analysis process in order to answer the research questions and fulfill thesis objectives.

The researcher used Nvivo queries to create the qualitative data displays. As the analysis of the raw data produced a large amount of data for this thesis, it was not feasible to present all the data and all the stages of analysis in detail. Instead, we focus on showing the data and the analysis that address the research questions and the objectives of this thesis. For the sake of transparency, we show all responses provided by respondents in the data tables. It should be noted, however, that not all the results displayed in the data tables are discussed in the text. This does not mean that the findings not discussed are unimportant, but instead that they are not what the researcher would view as critical to the objectives of the thesis. Readers are free, however, to peruse all the data and make their own judgements.

Four types of tables and one type of Venn diagram are used to display the qualitative results presented and discussed in this thesis. Examples of each of these four displays are shown in Table 6 (Total Sample), Table 7 (Sample by TS Stakeholder), Table 8 (Sample by Time: Cross-Sectional Data), and Table 9 (Sample by Time: Representative Panel Data), and discussed below. 


\begin{tabular}{|c|c|c|}
\hline Aggregate Theoretical Dimension & \multicolumn{2}{|c|}{$\begin{array}{c}\text { Total } \\
(n=75)\end{array}$} \\
\hline & $\#$ & $\%$ \\
\hline Second-order category 1 & 72 & 96 \\
\hline First-order code 1 & 44 & 59 \\
\hline First-order code 2 & 30 & 40 \\
\hline First-order code 3 & 21 & 28 \\
\hline Second-order category 2 & 10 & 13 \\
\hline First-order code 4 & 10 & 13 \\
\hline Second-order category 3 & 6 & 8 \\
\hline First-order code 5 & 6 & 8 \\
\hline
\end{tabular}

Note: Summation of First-order codes may not sum to Second-order category

Table 6: Example of first table type used to display results

As noted previously, first-order codes are abbreviations, or tags, for assigning concise meanings to a segment of descriptive data (Miles \& Huberman, 1984), which in this case come from our analysis of the interviewee responses. Second-order categories refer to a grouping of similar first-order codes, and are assigned to highlight cross-case patterns and to add elements of interpretation to the coding scheme (Miles \& Huberman, 1984). Aggregate theoretical dimensions refer to a grouping of similar second-order categories and aid in linking qualitative data to constructs within the theoretical framework. The first table type presents data (i.e., first-order codes, second-order categories, and the aggregate theoretical dimension) calculated using the total sample of respondents.

The first type of table (see Table 6) follows a particular row display format. The aggregate theoretical dimension being examined is provided in the top left corner of the table. The secondorder categories are each given a row of their own, marked with a shaded background. The firstorder codes (white background) are then presented in descending order under their respective second-order category, with those codes cited more often appearing first. 
Two types of numerical data are shown in the table columns: the number of respondents giving a particular response (column 2) and the percentage of the interviewees citing a particular response (column 3). Finally, it should be noted that the first-order codes might not always add up to the aggregated totals for the second-order categories, as one respondent may cite multiple first-order codes within the same second-order category.

The second table type (see Table 7 as an example) is used to present Time 1 or Time 2 data in a way that allows for cross-stakeholder data analysis. The same row display format used for the first table type is also used for the second table type. There are eight columns in the second table type. Column 1 provides labels for the first and second order codes representing the theoretical dimension under study. Columns 2 and 3 show the number of interviewees for TS Stakeholder 1 giving a particular response, and the percentage of the interviewees for TS Stakeholder 1 that cited a given response. Columns 4 and 5, and columns 6 and 7, provide these data for TS Stakeholder 2 and TS Stakeholder 3, respectively. It should be noted that perceptions (i.e. first-order codes) are only included in this table when they are mentioned by at least $10 \%$ of a TS stakeholder. Column 8 provides labels describing the extent that employees' perceptions exhibit 'sharedness' across stakeholder boundaries. The procedure taken to classify perceptions within the 'sharedness' construct is shown in the decision tree provided in Figure 7 and discussed in more detail below. 


\begin{tabular}{|c|c|c|c|c|c|c|c|}
\hline \multirow{2}{*}{ Aggregate Theoretical Dimension } & \multicolumn{2}{|c|}{ Stakeholder 1} & \multicolumn{2}{|c|}{ Stakeholder 2} & \multicolumn{2}{|c|}{ Stakeholder 3} & \multirow[t]{2}{*}{ 'Sharedness' Exhibited } \\
\hline & \# & $\%$ & \# & $\%$ & \# & $\%$ & \\
\hline Second-order category 1 & 24 & 96 & 24 & 96 & 22 & 88 & Strong Common \\
\hline First-order code 1 & 7 & 27 & 4 & 16 & 11 & 44 & $\begin{array}{c}\text { Weak Unique in Shared Situation (Stk } \\
\text { 3) }\end{array}$ \\
\hline First-order code 2 & 9 & 36 & 11 & 44 & 8 & 32 & Weak Common \\
\hline First-order code 3 & 18 & 72 & 19 & 76 & 11 & 44 & Strong Shared (Stk 1, Stk 2) \\
\hline Second-order category 2 & 0 & o & $\mathbf{0}$ & 0 & 10 & 40 & Strong Unique (Stk 3) \\
\hline First-order code 4 & 0 & 0 & 0 & 0 & 10 & 40 & Strong Unique (Stk 3) \\
\hline Second-order category 3 & 6 & 24 & $\mathbf{0}$ & $\mathbf{0}$ & $\mathbf{0}$ & $\mathbf{0}$ & Weak Unique (Stk 1) \\
\hline First-order code 5 & 6 & 24 & 0 & 0 & 0 & 0 & Weak Unique (Stk 1) \\
\hline
\end{tabular}

Note. Stk $=$ TS stakeholder.

Table 7: Example of second table type used to display results 


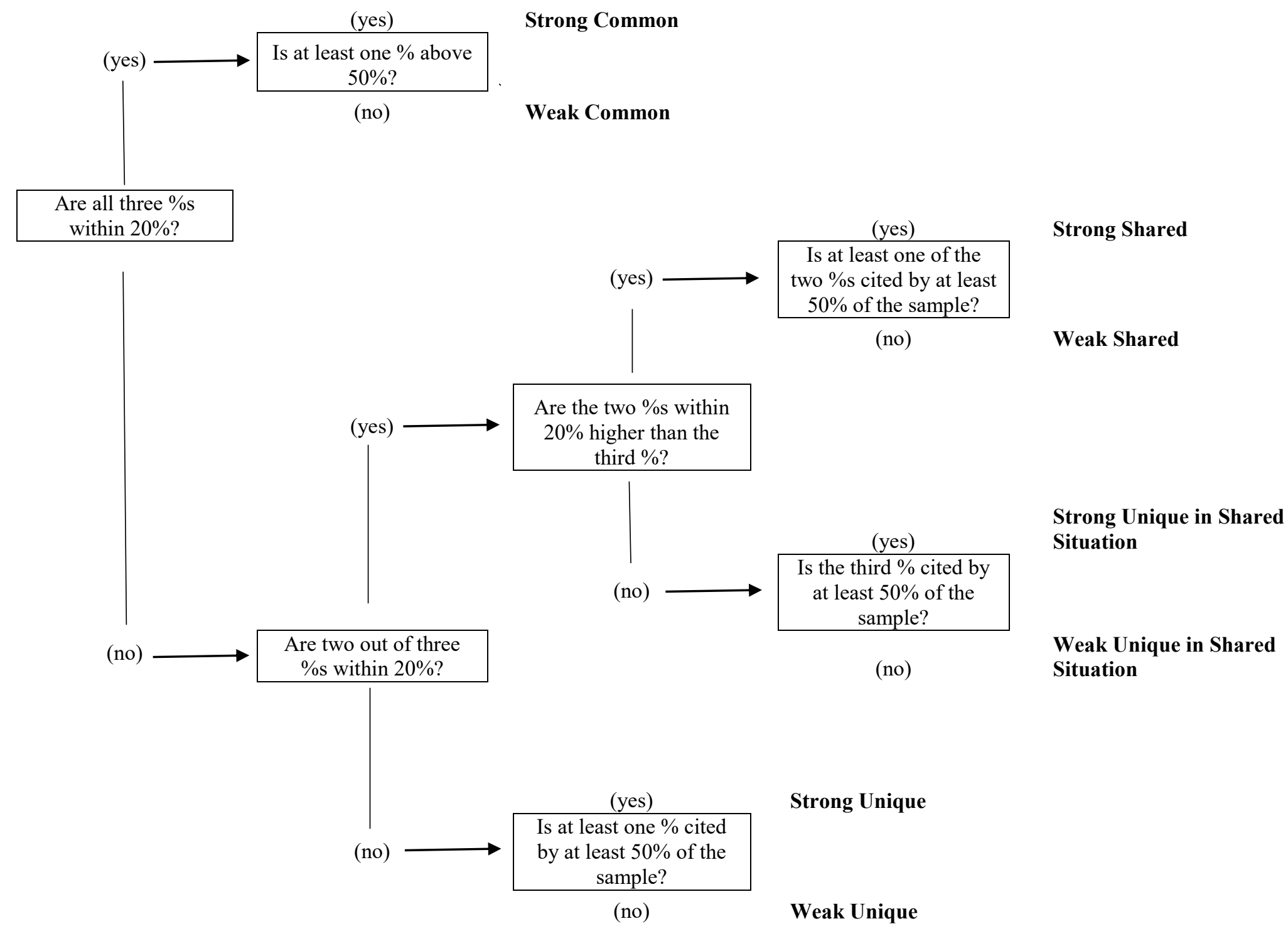

Note. \% refers to the percentage of respondents from each TS stakeholder who made a comment that we grouped under a particular first-order code.

Figure 7: Decision tree used classify first-order codes based on 'sharedness' construct 
The first step in operationalizing a perception within our 'sharedness' construct was to determine whether it was a common perception. If the percentage of respondents who cited a particular response from all three TS stakeholders were within $20 \%$, we considered this perception 'common'. For those perceptions cited by at least $50 \%$ of the respondents from each TS stakeholder, we considered them a strong view (i.e. strong common). All other common views we considered weak common.

If all TS stakeholders did not commonly hold the perception, we next examined whether the perception would be classified as unique. If the percentage of respondents who cited a particular response was not within $20 \%$ for two TS stakeholders, we considered the perception 'unique'. Those unique views cited by at least $50 \%$ of the respondents from a TS stakeholder were considered strong unique, while all others were considered weak unique.

Perceptions may also be considered unique if the percentage of respondents who cited a particular response from one TS stakeholder was more than $20 \%$ higher than the percentage of respondents who cited a particular response from either other TS stakeholder. In this case, we considered these unique perceptions in a shared situation. For this type of unique perception, views cited by at least $50 \%$ of the respondents in one TS stakeholder are considered 'strong unique in shared situations', while views cited by fewer than $50 \%$ of the respondents in one TS stakeholder are considered 'weak unique in shared situations.

Lastly, perceptions were classified as 'shared' if the percentage of respondents who cited a particular response from one TS stakeholder was within $20 \%$ of that of another TS stakeholder, and the percentage was higher than the percentage of respondents who cited that response from the third TS stakeholder. For those shared perceptions, views were classified as 'strong shared' 
when at least $50 \%$ of the respondents cited that response, while they were classified as 'weak shared' when less than $50 \%$ of the respondents cited that response. Finally, we acknowledge that the criteria used to classify 'sharedness' is somewhat arbitrary. It does, however, give us an appreciation of which views are shared and which are not, and how strongly views are shared.

The third table type (see Table 8 as an example) presents 'sharedness' data at Time 1 and Time 2 based on cross-sectional data so that we can see how 'sharedness' changes over time. There are three columns within this table. The first column provides labels for first-order codes. Columns 2 provides labels describing the extent that employees' perceptions exhibit 'sharedness' across stakeholder boundaries at Time 1. Column three presents the same type of data as column two but for Time 2. We consider shift in 'sharedness' of a particular perception (i.e. a first-order code) when the 'sharedness' label provided at Time 1 is different from the 'sharedness' label provided at Time 2. It should be noted that we only include first-order codes in this table if they have a common, shared, or unique label provided at either Time 1 or Time 2.

\begin{tabular}{|cc|c|c|}
\hline Aggregate Theoretical Dimension Over Time & $\begin{array}{c}\text { Pre-Change } \\
\text { Perception (Common } \\
\text { / Shared / Unique) }\end{array}$ & $\begin{array}{c}\text { Post-Change } \\
\text { Perception } \\
\text { (Common / Shared } \\
\text { / Unique) }\end{array}$ \\
\hline Second-Order Category & $\begin{array}{c}\text { Strong Shared (Org1, } \\
\text { Org2) }\end{array}$ & $\begin{array}{c}\text { Strong Shared (Org1, } \\
\text { Org2) }\end{array}$ \\
\hline First-Order Code 1 & Strong Shared (Org1, Org2) & $\begin{array}{c}\text { Strong Shared (Org1, } \\
\text { Org2) }\end{array}$ \\
\hline First-Order Code 2 & Weak Common & $\begin{array}{c}\text { Weak Shared (Org1, } \\
\text { Org2) }\end{array}$ \\
\hline Second-Order Category & Strong Common & $\begin{array}{c}\text { Strong Shared (Org2, } \\
\text { Org3) }\end{array}$ \\
\hline & First-Order Code 3 & Strong Common & $\begin{array}{c}\text { Strong Shared (Org2, } \\
\text { Org3) }\end{array}$ \\
\hline First-Order Code 4 & Weak Common & Weak Common \\
\hline First-Order Code 5 & Weak Unique (Org2) & Weak Unique (Org2) \\
\hline Second-Order Category & Strong Unique (Org3) & Strong Unique (Org3) \\
\hline & First-Order Code 6 & Strong Unique (Org3) & Strong Unique (Org3) \\
\hline First-Order Code 7 & Weak Unique (Org3) & Weak Unique (Org3) \\
\hline
\end{tabular}

Table 8: Example of third table type used to display results 
Table 9 shows the final table type, which was designed to facilitate comparison of perceptions within the TS at Time 1 to perceptions within the TS at Time 2 based on representative panel data. It should be noted that this table type only compares data from two stakeholders: hospital and police. Further information on why data from only two stakeholders are compared in this table type are provided in Section 4.8.

There are four columns within this fourth type of table. The first column provides labels for first-order codes. The second column specifies whether a particular response was provided at Time 1 or Time 2. The third and fourth columns show the number of respondents (and in brackets the percentage of respondents) from Stakeholder 1 and 2, respectively, that provided a particular response in either Time 1 or Time 2 interviews. We consider a change in perception when a particular response (i.e. first-order code) was mentioned by at least five respondents per TS stakeholder (i.e. $25 \%$ of TS stakeholder sample) either: (1) at Time 1 but not at Time 2, or (2) at Time 2 but not at Time 1 . Again, we note that this $25 \%$ cut off is somewhat arbitrary. We feel, however, that is a high enough percent of the sample to reduce the inclusion of spurious findings while at the same time making the analysis to be presented and discussed in this thesis manageable. It should also be noted that, while previous table types present data from all first-order codes, this table type only presents data from first-order codes that reflect a change in perception. In so doing, this table type allows us to focus our analysis on those perceptions that we believe 'changed' over time. 


\begin{tabular}{|c|c|c|c|}
\hline Aggregate Theoretical Dimension & $\begin{array}{l}\text { Perception } \\
\text { mentioned }\end{array}$ & $\begin{array}{c}\text { Stk 1 } \\
(n=20)\end{array}$ & $\begin{array}{c}\text { Stk 2 } \\
(n=20)\end{array}$ \\
\hline \multicolumn{4}{|l|}{ Second-Order Category 1} \\
\hline \multirow{2}{*}{ First-Order Code 1} & $\begin{array}{c}\text { Said T1 } \\
\text { not T2 } \\
\end{array}$ & $\begin{array}{c}6 \\
(30 \%) \\
\end{array}$ & $\begin{array}{c}0 \\
(0 \%) \\
\end{array}$ \\
\hline & $\begin{array}{c}\text { Said T2 } \\
\text { not T1 }\end{array}$ & $\begin{array}{c}1 \\
(5 \%)\end{array}$ & $\begin{array}{c}1 \\
(5 \%) \\
\end{array}$ \\
\hline \multicolumn{4}{|l|}{ Second-Order Category 2} \\
\hline \multirow{2}{*}{ First-Order Code 2} & $\begin{array}{l}\text { Said T1 } \\
\text { not T2 }\end{array}$ & $\begin{array}{c}7 \\
(35 \%)\end{array}$ & $\begin{array}{c}2 \\
(10 \%)\end{array}$ \\
\hline & $\begin{array}{c}\text { Said T2 } \\
\text { not T1 }\end{array}$ & $\begin{array}{c}6 \\
(30 \%)\end{array}$ & $\begin{array}{c}1 \\
(5 \%)\end{array}$ \\
\hline \multicolumn{4}{|l|}{ Second-Order Category 3} \\
\hline \multirow{2}{*}{ First-Order Code 3} & $\begin{array}{l}\text { Said T1 } \\
\text { not T2 }\end{array}$ & $\begin{array}{c}0 \\
(0 \%)\end{array}$ & $\begin{array}{c}10 \\
(50 \%)\end{array}$ \\
\hline & $\begin{array}{l}\text { Said T2 } \\
\text { not T1 }\end{array}$ & $\begin{array}{c}0 \\
(0 \%)\end{array}$ & $\begin{array}{c}10 \\
(50 \%)\end{array}$ \\
\hline
\end{tabular}

Note. $\mathrm{T} 1=$ Time $1 ; \mathrm{T} 2=$ Time 2. Stk $=$ TS stakeholder. Shaded cells identify changes in perceptions.

Table 9: Example of fourth table type used to display results

\subsubsection{INTERPRETING THE RESULTS}

The presentation of criteria for interpreting case study data is one of the final key components to this research design. Although there is no precise way to interpret findings in case study data analysis (Yin, 2009), proponents of case study and qualitative research specifically recommend that findings be judged by their substantive significance (Miles \& Huberman, 1994; Patton, 2002; Yin, 2009). Attaching significance to what has been found allows the researcher to then offer explanations, draw conclusions, and extrapolate lessons (Patton, 2002). The argument for substantive significance is made by presenting findings and conclusions that are judged to deepen the understanding of the phenomenon, to be sound and consistent in support of the findings, and to relate to other findings in the study (Patton, 2002). 
Case study researchers provide three cautions to interpreting qualitative case study data. First, Miles and Huberman (1994) warn against quantitative modes of thinking when interpreting data displays showing frequency counts. Second, Yin (2009) suggests that speculating on subtle patterns be avoided in favor of gross patterns whose interpretations are less likely to be challenged. Third, statistical techniques are inappropriate where samples have been purposefully selected to support theory development (Yin, 2009), as is the case in this research.

In this research, the interpretation of results was primarily based on data displays similar to the examples provided in Table 7, Table 8, and Table 9. The second table type allows frequency counts to be compared across TS stakeholders, the third table type allows 'sharedness' labels to be compared across time, and the fourth table type allows frequency counts to be compared across time for each TS stakeholder. Such displays are a convenient way to view the masses of data that qualitative analysis produces and allows the researcher to identify constructs and seek patterns and connections in the data that are important to the research objectives of this research. Presenting the coded data in tables also allow readers to make their own judgements about patterns in the data, which may support the researcher's interpretation of the data.

On a final note about interpretation, judgements about the importance of emerging relationships identified in qualitative data are based on substantive rather than statistical significance. In this thesis a great deal of effort was made to describe only the relative ranking of frequency counts and percentages between TS stakeholders for specific concepts, as recommended by Miles and Huberman (1994), and to avoid using language that infers statistical inference. When comparing results across TS stakeholders, however, the volume of data and the complexity of the planned TS change as described by the respondents often means that the narrative required to 
explain differences between TS stakeholders can be awkward, lengthy, and complex. To simplify the writing, when a particular concept is mentioned more often by one TS stakeholder than another, it is often easier and more meaningful to say that the concept is more likely to be cited by one TS stakeholder than by the others. The reader should keep in mind, however, that wherever the phraseology 'more or less likely' has been used in this thesis, it is not intended to infer statistical significance. Rather these terms are used to make the results and discussion sections more readable.

\subsubsection{TRIANGULATION}

Theoretically, triangulation is similar to cross-case analysis except that instead of comparing data by cases, the researcher compares data by data source (Eisenhardt, 1989). This strategy exploits unique insights possible from different types of data collection. Eisenhardt (1989, p.541) argues:

"when a pattern from one data source is corroborated by the evidence from another, the finding is stronger and better grounded. When evidence conflicts, the researcher can sometimes reconcile the evidence through deeper probing of the meaning of the differences."

Triangulation made possible by multiple data collection methods provides stronger substantiation of constructs and hypotheses (Eisenhardt, 1989). However, triangulation of data can be difficult to practically implement (Perlesz \& Lindsay, 2003). The following section describes how interview data may be triangulated with quantitative data, which in the case of this thesis comes from archival records.

\subsubsection{TRIANGULATION THROUGH QUANTITATIVE DATA}


As discussed earlier in this thesis, quantitative data can be used to complement qualitative data in case study research. Theory building case study research often uses quantitative data as an additional source of data to triangulate qualitative data at the case level (Johnson et al., 2007). In this research, the quantitative data reinforces and verifies the themes and content provided by the organizations in the qualitative data.

For example, the police record the length of time that police officers are waiting at the hospital for a physician to assess their apprehended PMI, and have summarized this data in a spreadsheet that shows the average police wait time per month. Over the course of the case study, the length of time police officers wait in the ED may change as a result of the implementation of planned TS changes. These archival records may allow us to identify actual changes in wait times between Time 1 and Time 2 (i.e. by comparing wait times before changes were implemented to wait times six months after changes were implemented). We can then compare these quantitative findings to perceptions of what has changed in the TS (at Time 2) in order to get a better understanding of how TS change unfolds over time in response to a planned TS change. This form of triangulation is particularly important as it allows the researcher to explore changes in perceptions of each TS stakeholder by: (1) comparing TS stakeholders' perceptions to unbiased archival data, and (2) investigating the notion that one TS stakeholder's perceptions may be closer to 'reality' than that of another TS stakeholder.

\subsection{METHODOLOGY IN PRACTICE}

Throughout this chapter, we have described our approach to conducting a rigorous research study that involves collecting and analyzing data from multiple organizations (i.e. Police, Hospital, and Social Service Agency) over multiple time periods (i.e. Time 1 and Time 2). It is rare, 
however, that the practice of longitudinal research always goes as planned (Eisenhardt \& Graebner, 2007; Yin, 2009). This section describes the sample demographics of respondents at Time 1 (Section 4.8.1) and at Time 2 (Section 4.8.2). It also discusses the implications on data analysis and theory development of having a number of respondents drop out of the study (Section 4.8.3).

\subsubsection{DESCRIPTION OF TIME 1 SAMPLE}

Time 1 interviews were conducted during the fall of 2013. Table 10 shows the gender, job type, and frequency with which respondents interacted with PMIs for interviewees belonging to each of the three TS stakeholders at Time 1.

\begin{tabular}{|c|c|c|c|c|}
\hline Time 1 & Hospital & Police & $\begin{array}{c}\text { Social } \\
\text { Service } \\
\text { Agency } \\
\end{array}$ & Total \\
\hline \multicolumn{5}{|l|}{ Gender } \\
\hline Male & $12(48 \%)$ & $22(88 \%)$ & $11(44 \%)$ & $45(60 \%)$ \\
\hline Female & $13(52 \%)$ & $3(12 \%)$ & $14(56 \%)$ & $30(40 \%)$ \\
\hline \multicolumn{5}{|l|}{ Job Type } \\
\hline Manager (e.g. Sergeant, Doctor, Supervisor) & $9(36 \%)$ & $7(28 \%)$ & $0(0 \%)$ & $16(21 \%)$ \\
\hline Frontline (e.g. Constable, nurse, social worker) & $16(64 \%)$ & $18(72 \%)$ & $25(100 \%)$ & $59(79 \%)$ \\
\hline \multicolumn{5}{|l|}{ Frequency interacting with PMIs } \\
\hline Hourly & $6(24 \%)$ & $0(0 \%)$ & $10(40 \%)$ & $16(21 \%)$ \\
\hline Daily & $14(56 \%)$ & $15(60 \%)$ & $15(60 \%)$ & $44(59 \%)$ \\
\hline Weekly & $4(16 \%)$ & $7(28 \%)$ & $0(0 \%)$ & $11(15 \%)$ \\
\hline Monthly & $1(4 \%)$ & $3(12 \%)$ & $0(0 \%)$ & $4(5 \%)$ \\
\hline Total & 25 & 25 & 25 & 75 \\
\hline
\end{tabular}

Table 10: Time 1 Interviewee Demographics

Slightly more males than females participated in our Time 1 interviews ( $60 \%$ male). This was largely due to the number of male police officers (22 out of 25) interviewed at Time 1 . The Hospital (48\% male) and the Social Service Agency (44\% male) samples were fairly equally distributed in terms of the number of men and women interviewed. 
Over three-quarters (79\% frontline) of the interviewees at Time 1 were frontline workers, while the remainder were managers. Within the Hospital sample, $64 \%$ of the people interviewed were frontline workers such as Hospital administrators, ED triage nurses, ED nurses, ED resource nurses, or security guards, while the remainder acted in a managerial role such as an ED physician, a MHESU psychiatrist, a supervisor, or a manager. The Police sample consisted of $72 \%$ frontline workers (i.e. constables) and 28\% managers (i.e. sergeant, staff sergeant). All Social Service Agency employees interviewed at Time 1 were frontline staff (i.e., community support workers, nurses) working for either the Mental Health and Justice team or the Assertive Community Treatment (ACT) team.

Over half (59\%) of the total Time 1 sample interacted with PMIs daily and only four respondents (mainly sergeants in the Police sample) interacted with PMIs monthly. It appears that respondents from the Social Service Agency organization interacted with PMIs more frequently than their counterparts in other organizations $-40 \%$ of the Social Service Agency sample interacted hourly and $60 \%$ of the Social Service Agency sample interacted daily with PMIs. The Police sample, on the other hand, consisted of respondents who interacted with PMIs daily (60\%), weekly $(28 \%)$, or monthly $(12 \%)$.

\subsubsection{DESCRIPTION OF TIME 2 SAMPLE}

A second round of interviews (i.e. Time 2 interviews) took place over a three-month period in winter 2015. These interviews happened approximately one year after the first set of interviews, and six months after the planned changes were implemented.

It was not possible to schedule a second interview with all the respondents that were interviewed at Time 1. Fifteen respondents no longer worked for the same TS stakeholder, six had 
transitioned within their TS stakeholder and no longer deal with the PMI process, and five were on long-term leave (e.g. maternity leave). Table 11 presents the sample of those people from the first round of interviews who were interviewed for a second time. To help the reader, the number without brackets refers to the number of individuals who were interviewed at both Time 1 and Time 2, whereas the number in brackets refers to the number of individuals who were interviewed prior to the implementation of change (i.e. Time 1). Finally, the percentages in Table 11 refer to the proportion of respondents who participated in both Time 1 and Time 2 interviews.

Examination of the data found in Table 11 reveals three important findings that impact our ability to draw meaningful conclusions from changes to perceptions over time. First, as eighty percent of the respondents interviewed at Time 1 from the Hospital were also interviewed at Time 2, we have confidence in our ability to examine planned TS change over time based on the perceptions of healthcare workers. Second, given that eighty percent of the police officers interviewed at Time 1 were also interviewed at Time 2, we have confidence in our ability to explore planned TS change over time based on police officers' perceptions. 


\begin{tabular}{|c|c|c|c|c|}
\hline Time 2 & Hospital & Police & $\begin{array}{c}\text { Social } \\
\text { Service } \\
\text { Agency }\end{array}$ & Total \\
\hline \multicolumn{5}{|l|}{ Gender } \\
\hline Male & $\begin{array}{c}9(12) \\
75 \% \\
\end{array}$ & $\begin{array}{c}19(22) \\
86 \% \\
\end{array}$ & $\begin{array}{c}2(11) \\
18 \%\end{array}$ & $\begin{array}{c}30(45) \\
67 \%\end{array}$ \\
\hline Female & $\begin{array}{c}11(13) \\
85 \% \\
\end{array}$ & $\begin{array}{l}1(3) \\
33 \% \\
\end{array}$ & $\begin{array}{c}7(14) \\
50 \% \\
\end{array}$ & $\begin{array}{c}19(30) \\
63 \% \\
\end{array}$ \\
\hline \multicolumn{5}{|l|}{ Job Type } \\
\hline Manager (e.g. Sergeant, Doctor, Supervisor) & $\begin{array}{l}7(9) \\
78 \%\end{array}$ & $\begin{array}{l}6(7) \\
86 \%\end{array}$ & $\begin{array}{c}0(0) \\
0 \%\end{array}$ & $\begin{array}{c}13(16) \\
81 \%\end{array}$ \\
\hline Frontline (e.g. Constable, nurse, social worker) & $\begin{array}{c}13(16) \\
81 \% \\
\end{array}$ & $\begin{array}{c}14(18) \\
78 \% \\
\end{array}$ & $\begin{array}{c}9(25) \\
36 \% \\
\end{array}$ & $\begin{array}{c}36(59) \\
61 \% \\
\end{array}$ \\
\hline \multicolumn{5}{|l|}{ Frequency interacting with PMIs } \\
\hline Hourly & $\begin{array}{l}5(6) \\
83 \% \\
\end{array}$ & $\begin{array}{c}0(0) \\
0 \% \\
\end{array}$ & $\begin{array}{c}4(10) \\
40 \% \\
\end{array}$ & $\begin{array}{c}9(16) \\
56 \% \\
\end{array}$ \\
\hline Daily & $\begin{array}{c}11(14) \\
79 \%\end{array}$ & $\begin{array}{c}14(15) \\
93 \%\end{array}$ & $\begin{array}{c}5(15) \\
33 \%\end{array}$ & $\begin{array}{c}30(44) \\
68 \%\end{array}$ \\
\hline Weekly & $\begin{array}{l}3(4) \\
75 \%\end{array}$ & $\begin{array}{l}5(7) \\
71 \%\end{array}$ & $\begin{array}{c}0(0) \\
0 \%\end{array}$ & $\begin{array}{c}8(11) \\
73 \%\end{array}$ \\
\hline Monthly & $\begin{array}{c}1(1) \\
100 \% \\
\end{array}$ & $\begin{array}{l}1(3) \\
33 \% \\
\end{array}$ & $\begin{array}{c}0(0) \\
0 \%\end{array}$ & $\begin{array}{l}2(4) \\
50 \%\end{array}$ \\
\hline Total & $\begin{array}{c}20(25) \\
80 \%\end{array}$ & $\begin{array}{c}20(25) \\
80 \%\end{array}$ & $\begin{array}{c}9(25) \\
36 \% \\
\end{array}$ & $\begin{array}{c}49(75) \\
65 \% \\
\end{array}$ \\
\hline
\end{tabular}

Table 11: Demographics of respondents interviewed for a second time

Third, unfortunately, only 9 of the original 25 Social Service Agency employees who participated in the Time 1 interviews could be contacted and were available to be interviewed a second time. This may have been because there were significant internal changes taking place for the Social Service Agency organization between Time 1 and Time 2 including a change in CEO, a change in $\mathrm{COO}$, and a significant turnover of employees as frontline workers followed their senior leaders who left the organization. To compensate for the decreased number of Social Service Agency employees available to be interviewed at Time 2, we recruited an additional 11 Social Service Agency employees for the Time 2 interview process. These 11 individuals all worked for the Social Service Agency at Time 1 and had frequent interactions with the PMI process and their partnering TS stakeholders.

Table 12 below shows the demographics of the Social Service Agency employees who participated in each round of interviews. Sixteen employees participated in only Time 1 
interviews, nine participated in both Time 1 and Time 2 interviews, and eleven participated in only Time 2 interviews. The demographics of the employees interviewed at each of these time periods show some similarity. First, the majority of the Social Service Agency employees interviewed at each point in time were frontline workers (only 2 of the 11 new employees interviewed at Time 2 were managers). Second, the majority of Social Service Agency employees interviewed at each point in time interacted with PMIs daily.

\begin{tabular}{|c|c|c|c|}
\hline \multirow[b]{2}{*}{ Interview Round Participated In } & \multicolumn{3}{|c|}{ Social Service Agency } \\
\hline & $\begin{array}{c}\text { Only } \\
\text { Time } 1\end{array}$ & $\begin{array}{c}\text { Time } 1 \text { and } \\
\text { Time } 2\end{array}$ & $\begin{array}{c}\text { Only } \\
\text { Time } 2\end{array}$ \\
\hline \multicolumn{4}{|l|}{ Gender } \\
\hline Male & 9 & 2 & 3 \\
\hline Female & 7 & 7 & 8 \\
\hline \multicolumn{4}{|l|}{ Job Type } \\
\hline Manager & 0 & 0 & 2 \\
\hline Frontline (e.g. community support worker, nurse) & 16 & 9 & 9 \\
\hline \multicolumn{4}{|l|}{ Frequency interacting with PMIs } \\
\hline Hourly & 6 & 4 & 4 \\
\hline Daily & 10 & 5 & 6 \\
\hline Weekly & 0 & 0 & 1 \\
\hline Monthly & 0 & 0 & 0 \\
\hline Total & 16 & 9 & 11 \\
\hline
\end{tabular}

Table 12: Demographics of Social Service Agency employees by time of interview

We were faced with two options with respect to how we could analyze the Social Service Agency data: (1) include the data from the 9 Social Service Agency respondents who interviewed at Time 1 and at Time 2 within our analysis of panel data, or (2) include the data from the 20 Social Service Agency respondents who interviewed at Time 1 and the data from the 20 Social Service agency respondents who were interviewed at Time 2 within our cross-sectional data analysis. We 
decided to use the second approach (cross-sectional data). The first approach involved an analysis with a very small $\mathrm{n}$ (i.e. 9 respondents), which limited our ability to draw conclusions on causality. The demographic similarities between the Time 1 and Time 2 Social Service Agency samples gives us further confidence in the appropriateness of this approach.

Table 13 provides information on the total interview sample. The table shows the number of people interviewed in each round of data collection. The column for Time 2 contains two numbers: the number not in brackets refers to the number of individuals interviewed at Time 2, while the number in brackets refers to the number of individuals interviewed at Time 2 that were also interviewed at Time 1. In all, 75 people were interviewed at Time 1, 60 were interviewed at Time 2, and 49 were interviewed at both time periods.

\begin{tabular}{|c|c|c|c|c|c|c|c|c|}
\hline \multirow{2}{*}{ Complete Sample } & \multicolumn{2}{|c|}{$\begin{array}{l}\text { Hospital } \\
(n=20)\end{array}$} & \multicolumn{2}{|c|}{$\begin{array}{c}\text { Police } \\
(n=20)\end{array}$} & \multicolumn{2}{|c|}{$\begin{array}{c}\text { Social Service } \\
\text { Agency } \\
(\mathbf{n}=\mathbf{2 0})\end{array}$} & \multicolumn{2}{|c|}{$\begin{array}{c}\text { Total } \\
(n=60)\end{array}$} \\
\hline & Time 1 & $\begin{array}{c}\text { Time } 2 \\
\text { (Time 1 \& } \\
\text { Time 2) } \\
\end{array}$ & Time 1 & $\begin{array}{l}\text { Time } 2 \\
\text { (Time } 1 \& \\
\text { Time 2) } \\
\end{array}$ & Time 1 & $\begin{array}{l}\text { Time } 2 \\
\text { (Time } 1 \& \\
\text { Time 2) } \\
\end{array}$ & Time 1 & $\begin{array}{c}\text { Time } 2 \\
\text { (Time 1 \& } \\
\text { Time 2) } \\
\end{array}$ \\
\hline \multicolumn{9}{|l|}{ Gender } \\
\hline Male & 12 & $9(9)$ & 22 & $19(19)$ & 11 & $5(2)$ & 45 & 33 (30) \\
\hline Female & 13 & $11(11)$ & 3 & $1(1)$ & 14 & $15(7)$ & 30 & 27 (19) \\
\hline \multicolumn{9}{|l|}{ Job Type } \\
\hline $\begin{array}{r}\text { Manager (e.g. Sergeant, } \\
\text { Doctor, Supervisor) }\end{array}$ & 9 & 7 (7) & 7 & $6(6)$ & 0 & $2(0)$ & 16 & $15(13)$ \\
\hline $\begin{array}{r}\text { Frontline (e.g. Constable, } \\
\text { nurse, social worker) }\end{array}$ & 16 & $13(13)$ & 18 & $14(14)$ & 25 & $18(9)$ & 59 & $45(36)$ \\
\hline \multicolumn{9}{|l|}{$\begin{array}{r}\text { Frequency interacting } \\
\text { with PMIs }\end{array}$} \\
\hline Hourly & 6 & $5(5)$ & 0 & $0(0)$ & 10 & $7(4)$ & 16 & $12(9)$ \\
\hline Daily & 14 & $11(11)$ & 15 & $14(14)$ & 15 & $12(5)$ & 44 & 37 (30) \\
\hline Weekly & 4 & $3(3)$ & 7 & $5(5)$ & 0 & $1(0)$ & 11 & $9(8)$ \\
\hline Monthly & 1 & $1(1)$ & 3 & $1(1)$ & 0 & $0(0)$ & 4 & $2(2)$ \\
\hline Total & 25 & $20(20)$ & 25 & $20(20)$ & 25 & $20(9)$ & 75 & $60(49)$ \\
\hline
\end{tabular}

Table 13: Summary of interviews carried out 
In summary, as only 9 Social Service Agency respondents participated in both sets of interviews, we do not have confidence to longitudinally analyze Social Service Agency data on an individual level. The fact that the Social Service Agency sample at Time 1 is demographically similar to that at Time 2 gives us confidence that the cross-sectional study of Social Service Agency respondents will provide us with some idea of how the planned TS changes impacted perceptions of TS stakeholder relationships at the group level. Given that $80 \%$ of the respondents $(n=20)$ in both the Hospital and Police samples participated in both rounds of interviews, we were able to collect a panel data set from Hospital and Police respondents that gives us confidence in our ability to analyse these two TS stakeholder samples at the individual level (i.e. compare responses at the level of the respondent). 


\section{CHAPTER 5: ANALYSIS OF THE RESULTS THROUGH A CHANGE MANAGEMENT LENS}

This chapter presents and discusses data that speak to the changes that were introduced within the TS as part of the planned change initiative. The chapter is divided into four sections, each of which provide us with a different perspective on the change. We begin in Section 5.1 with a brief summary of the events that occurred prior to the introduction of any changes within the TS. The objective criteria which were used by the stakeholders to evaluate the impact of the changes introduced as part of this initiative on outcomes of interest are outlined and discussed in Section 5.2. Section 5.3 presents key findings from the Time 1 (what respondents wanted to change within the TS) and Time 2 (what respondents observed had changed within the TS over time) that relate to the two change constructs in our theoretical framework (see Figure 6). In Section 5.4, we use sensemaking theory to help us understand how respondents made sense of the planned TS change. Finally, in Section 5.5 we draw from the change literature when discussing key findings from this chapter.

\subsection{WHAT CHANGED: THE REALITY OF THE PLANNED TS CHANGE}

The theoretical framework used to orient this study (see Section 3.1) explores two dimensions of change: (1) what stakeholders wanted changed within the TS prior to any changes being introduced, and (2) what stakeholders perceived had changed within the TS six months after the planned changes had been implemented. This chapter provides the reader with relevant information that contributes to our understanding of the planned TS change (see Figure 8) from multiple perspectives. The activities that led up to the launch of the planned TS change are presented first (Section 5.1.1). This is followed in Section 5.1.2 with a description of the changes 
that were implemented as part of the planned TS change along with the rationale behind their implementation.

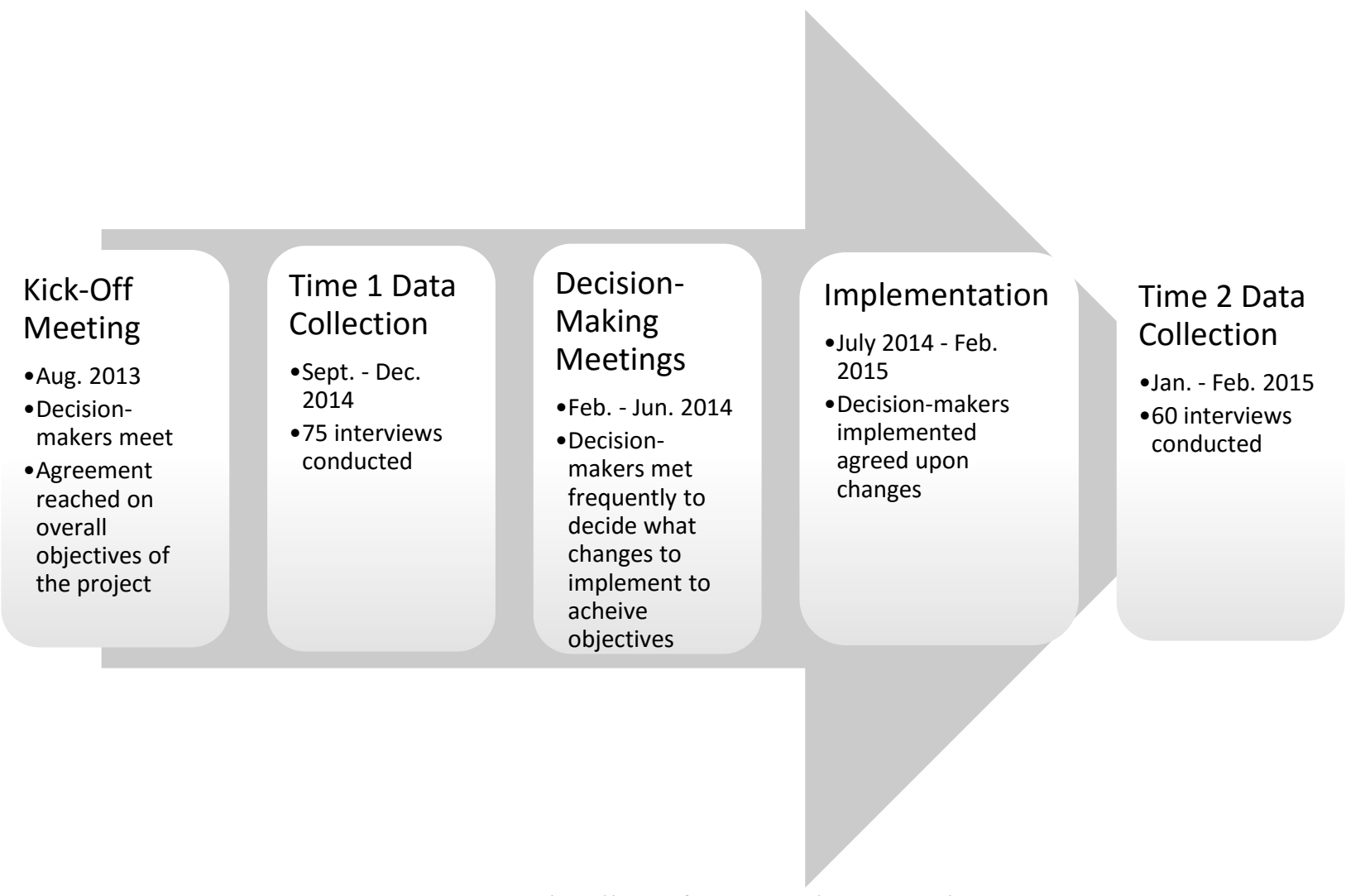

Figure 8: Timeline of case study research

\subsubsection{KICK-OFF MEETING}

In August of 2013 a meeting was held to discuss the planned TS change. Attendees included senior managers from each of the three organizations (i.e. decision-makers) within the TS, the researcher, and the researcher's supervisor. The purpose of the kick-off meeting was to: (1) formally introduce the senior managers to each other, (2) agree on the objective of the planned TS change, and (3) identify metrics that would be used to evaluate the success of any changes to the system over time. After initial introductions, the group quickly agreed that they needed to improve the relationships between the three organizations within the PMI process so that they 
could provide a better service to PMIs. After three hours of discussions, the group agreed that the first step in building these relationships would be to determine how best to decrease the amount of time that PMIs have to wait in the Hospital during the PMI process. To achieve this overarching objective, the group of decision-makers decided that they would utilize the following threepronged approach:

1) Identify and implement strategies to decrease the number of PMIs apprehended by Police and brought to the Hospital;

2) Identify and implement strategies to decrease the number of PMIs apprehended by Police, brought to the Hospital, and not formed; and

3) Identify and implement strategies to decrease the length of time PMIs apprehended by Police have to wait in the Hospital before being evaluated.

At the point in time the decision-makers identified these three goals, it was thought that the three objectives had the potential to benefit each of the three TS stakeholders. More specifically, the Hospital would primarily benefit from the first two objectives, as they could result in: (1) fewer PMIs entering the ED overall, and (2) fewer PMIs entering the ED who do not have legitimate reasons to be there. The Social Service Agency would also primarily benefit from the first two objectives, as they could result in fewer situations where PMIs have their rights removed by police officers. The Police would primarily benefit from objective three, as this could result in police officers spending less time in the Hospital.

The actual strategies used to achieve these three objectives were decided upon by the decision-makers between February and June 2014. How they made these decisions is outside the 
scope of this thesis (see Halinski \& Duxbury (2015) for details on the decision-making process).

The decisions on what was to be changed and why are described in detail in the following section.

\subsubsection{SPECIFIC CHANGES TO BE IMPLEMENTED}

The decision-makers agreed upon a set four specific changes that were to be implemented within the TS to achieve their three objectives: (1) police mental health training, (2) an In Short Term Emergency Deviation (InSted) program, (3) the mandatory completion of Emotionally Disturbed Person (EDP) forms by the Police, and (4) an escalation and intervention policy. Table 14, below, maps out each of these four specific changes (i.e. middle column) against the decisionmakers' three objectives that were outlined in the initial kick-off meeting (i.e. left-hand column), as well as a set of indicators (i.e. right-hand column) that were used to evaluate the success of the change. Details on each of these planned changes are provided in the sections below.

\begin{tabular}{|l|l|l|}
\hline \multicolumn{1}{|c|}{$\begin{array}{c}\text { Decision-Makers } \\
\text { Objectives } \\
\text { (see Section 5.1.1) }\end{array}$} & \multicolumn{1}{|c|}{$\begin{array}{c}\text { Changes Implemented } \\
\text { (see Section 5.1.2) }\end{array}$} & $\begin{array}{l}\text { Indicators Used to Evaluate } \\
\text { if Change resulted in } \\
\text { desired outcome } \\
\text { (see Section 5.2) }\end{array}$ \\
\hline $\begin{array}{l}\text { Decrease the number of PMIs } \\
\text { apprehended by the Police per } \\
\text { month }\end{array}$ & 1) InSted Program & $\begin{array}{l}\text { 1) Percentage of PMIs apprehended } \\
\text { by Police per month }\end{array}$ \\
\hline $\begin{array}{l}\text { Decrease the number of PMIs } \\
\text { apprehended by the Police and } \\
\text { not formed per month }\end{array}$ & $\begin{array}{l}\text { 2) Police Mental Health Training } \\
\text { 3) Mandatory Completion of EDP Forms }\end{array}$ & $\begin{array}{l}\text { 2) Percentage of EDP forms } \\
\text { completed by Police per month } \\
\text { 3) Percentage of PMIs apprehended } \\
\text { by Police and formed by physician } \\
\text { per month }\end{array}$ \\
\hline $\begin{array}{l}\text { Decrease average length of } \\
\text { time PMIs apprehended by the } \\
\begin{array}{l}\text { Police have to wait in the } \\
\text { Hospital before being } \\
\text { evaluated per month }\end{array}\end{array}$ & $\begin{array}{l}\text { 4) Average length of time PMIs } \\
\text { apprehended by Police have to wait } \\
\text { to be assessed by a physician per } \\
\text { month }\end{array}$ \\
\hline
\end{tabular}

Table 14: Mapping decision-makers' objectives to implemented changes and indicators used to evaluate change

\subsubsection{IN SHORT TERM EMERGENCY DEVIATION (InSted) PROGRAM}


Although most of the media attention related to the PMI process is devoted to challenges at the frontend of the process (e.g., police training, police apprehensions, police wait times), our analysis of the PMI process using Lean methodologies (outside the scope of this thesis) identified significant challenges that occur at the tail end of the PMI process. Most notably, when a PMI is released from the Hospital (either they weren't formed in the ED or they were discharged from the MHESU), s/he typically walks out of the Hospital without being connected to the necessary support services in the community (e.g., a shelter, a Social Service Agency worker). As a result, this discharged PMI often lacks the necessary community resources to cope with their illness. Without this support, the PMI is more likely to have a reoccurrence of their mental health issue and return to the Hospital after being apprehended by Police. This situation led the decisionmakers to expect that linking discharged PMIs to services in the community would improve the long-term care of these individuals and reduce recidivism (i.e., reduce PMI relapses, lessen the number of repeat apprehensions by Police).

To connect more discharged PMIs with services in the community, the group of decisionmakers elected to implement the In Short Term Emergency Deviation (InSted) Program in the Hospital. This program involved hiring a Social Service employee (i.e. the InSted worker) to work from within the MHESU. The primary role of the InSted worker is to connect "frequent flyers" (the label police and healthcare workers have given to PMIs who came into the ED at least three times in the past month), to the appropriate services in the community. InSted workers have complete access to the Social Service database, and thus can provide MHESU staff with more information to help them assess and treat the PMIs.

\subsubsection{POLICE MENTAL HEALTH TRAINING}


In recent decades, the number of interactions between PMIs and police officers has increased significantly across Canada (Cotton \& Coleman, 2010). While most of these interactions are minor in nature and are resolved uneventfully, some result in less than positive outcomes (e.g. Coleman \& Cotton, 2010). Police organizations are reacting to this situation by administering new mental health training programs, which provide their officers with the skillsets required to appropriately identify and apprehend PMIs who are endangering themselves and/or others (e.g. Cotton \& Coleman, 2010). This trend led the decision-makers to expect that improving the Police's mental health training program would give police officers a better understanding of mental health issues. This increase in knowledge would, it was hoped, lead to more accurate 'diagnosis' of potential mental health issues at the scene, which would, in turn, lower the number of PMIs brought to the hospital who were not formed (i.e. not presenting with a mental health issue). The decision-makers also expected that this decrease in demand would lead to an increase in the quality of care given to the PMI during an apprehension.

The Police agreed to change their mental health training program in two ways. First, the Police mandated that all officers who were responsible for bringing PMIs to the Hospital complete a 30-minute online mental health training module that was administered by Police management. This module focused on the presentation and conditions of individuals with mental illness. Second the Police also increased the number of in-person mental health training sessions from three sessions per year to four sessions per year. It should be noted that each session is offered to a different group of police officers and that, at the time when we collected Time 2 data, not all police officers had taken this program. 


\subsubsection{MANDATORY COMPLETION OF EMOTIONALLY DISTURBED PERSON (EDP) FORMS}

Even though the Police have nominally included the task of completing an Emotionally Disturbed Person (EDP) form in their policies and procedures associated with PMI apprehensions, they have seen a limited adoption of this practice. An EDP form is a sheet the apprehending police officer fills out to provide background information about the PMI to the attending ED physician and/or MHESU psychiatrist. The goal of this form is to provide doctors with as much information as possible so that they can properly assess (and potentially form) the PMI. Both Hospital and Police managers agreed that, before the implementation of the planned TS change, the EDP form was frequently not completed by police officers. The limited compliance in completing these forms was considered unfortunate, as it was felt that the information on this form might allow doctors to assess PMIs more accurately. The decision-makers expected that, by mandating police officers to complete EDP forms, they would increase the amount of relevant information available to the attending ED physician and/or MHESU psychiatrist. This information would, in turn, allow these doctors to assess (and potentially form) the PMIs more appropriately. The hospital would also give feedback to the officers who filled out the form as it was hoped that this feedback would increase the officers' ability to accurately determine which individuals to bring to the hospital.

The following changes were institutionalized to increase the number of police officers completing EDP forms. First, the Police started printing EDP forms on carbon copy paper so that two copies would be produced when the police officer filled out the form. One completed form would be left at the Hospital so that the doctor could use it in their assessment of the PMI while the second would be brought back to the Police service to ensure that the officers were filling them 
out. Next, the police officers would be required to have their supervisor on duty sign off their copy of the form, a requirement that was expected to increase the compliance of police officers filling out the EDP form. Lastly, the signed off Police copy would be sent to the Police's mental health coordinator for assessment, so as to increase feedback from the frontline workers filling out the EDP forms to senior Police managers who are developing these policies.

\subsubsection{ESCALATION AND INTERVENTION POLICY}

Police services across Canada are challenged by the amount of resources that are consumed by police officers waiting in hospitals for doctors to assess apprehended PMIs (Coleman \& Cotton, 2010). This waiting period stems from the fact that, under the mental health act, police officers are obligated to remain at the hospital with the PMI until the custody of the PMI is transferred from the Police to the Hospital. Throughout this research, it was understood that this transfer of custody occurred when a physician assesses (i.e. forms or releases) the PMI. As police officers were obligated to remain at the Hospital until a doctor assessed the apprehended PMI, the decision-

makers expected that implementing a strategy within the Hospital that addressed when doctors assessed those PMIs who were brought to the Hospital by the Police would decrease the amount of time police officers were spending in the Hospital.

The Hospital and Police took several steps to decrease the amount of time police officers were spending in the Hospital. First, a memorandum of understanding (MOU) was created to delineate expectations for the Hospital and the Police when participating in the PMI process. Police and Hospital management agreed that Hospital staff would target a 2 hour wait time for the physician's initial assessment of any PMI brought into the Hospital by the Police. They also agreed that in those cases where the PMI had not been seen by a physician within a 2-hour time period, 
frontline police officers would contact their supervisor, and the supervisor would call the ED resource nurse to remind them that police officers have been waiting 2 hours. The resource nurse would then prioritize the apprehended PMI within the cue of patients waiting to see the doctor, and verbally communicate that police officers had been waiting over two hours to see the physician. It was expected that increased prioritization and verbal communication should remind the physician that they needed to assess the PMI next. Second, a special telephone was installed in the ED so that police officers could call Hospital staff to let them know that they had apprehended a PMI and were approaching the Hospital with a PMI requiring assessment. This practice has been used in other Police - Hospital collaborations and is based on the supposition that such a call would allow the Hospital to prepare resources (e.g., beds, nurses) for incoming PMIs, which would expedite the processing of the PMI and reduce police wait times.

In this thesis, the term planned TS change refers to these four sets of changes (i.e., Police mental health training, InSted Program, mandatory completion of EDP form, and escalation and intervention policy). The next section analyzes quantitative data to determine the extent to which the implementation of the planned TS change in fact changed behavior within the TS.

\subsection{WHAT CHANGED: AN OBJECTIVE ANALYSIS OF THE PLANNED TS CHANGE}

The decision-makers identified four indicators that they felt would allow them to identify the extent to which the planned TS change had resulted in the desired outcomes: (1) percentage of PMIs apprehended by Police per month, (2) percentage of EDP forms completed by Police per month, (3) percentage of PMIs apprehended by Police and formed by a physician per month, and (4) the average length of time PMIs apprehended by Police have to wait to be assessed by a physician per month. This section begins with the presentation of data associated with each of the 
four indicators (Section 5.2.1 through Section 5.2.4) and ends with a summary of key findings from this stage of the analysis (Section 5.2.5).

\subsubsection{PERCENTAGE OF PMIs APPREHENDED BY POLICE PER MONTH}

The percentage of PMIs apprehended by Police per month was calculated by dividing the number of PMI apprehensions per month by the number of Police - PMI interactions per month. A PMI apprehension is defined as occurring when a police officer interacts with a PMI in the community and brings the PMI involuntarily to the hospital under the mental health act. A Police - PMI interaction, on the other hand, is defined as occurring when a police officer interacts with a PMI in the community and records their interaction in their database. Percentage of PMIs apprehended by police officers per month data are shown in Table 15. Each month's percentage reflects the likelihood that police officers would apprehend PMIs they interacted with during that month. The data are presented in a format that allows us to easily compare the percentage of police officers apprehending PMIs they interact with in the community (i.e. "percent apprehended") in a particular month to the percent apprehended in the same month one year later (i.e. compare May 2014 data with May 2015 data). Data collected in the months after the planned TS change was implemented are indicated with the grey shading. Examination of the data in Table 15 indicates that the "percent apprehended" prior to the introduction of the planned TS change is lower than the "percent apprehended post change". In other words, there has been an increase in the percentage of PMIs apprehended by the Police per month post-implementation of the planned TS change. Based on our analysis, these findings may suggest that the InSted program has not reduced the number of PMIs brought to the Hospital. 


\begin{tabular}{|c|c|c|c|c|c|c|c|c|c|c|c|c|}
\hline & 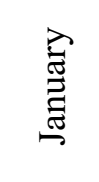 & 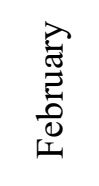 & 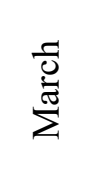 & $\overline{\vec{Z}}$ & $\stackrel{\overrightarrow{\mathrm{J}}}{\mathrm{\lambda}}$ & $\stackrel{\Xi}{\Xi}$ & $\stackrel{\gtrsim}{\Xi}$ & 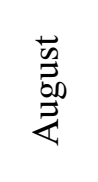 & 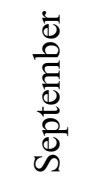 & $\begin{array}{l}\overline{0} \\
\stackrel{0}{0} \\
\stackrel{0}{0}\end{array}$ & $\begin{array}{l}\dot{\bar{d}} \\
\ddot{Z} \\
\overline{0} \\
z \\
z \\
z\end{array}$ & 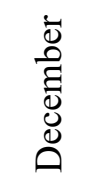 \\
\hline 2013 & & & & & & & & & $51 \%$ & $53 \%$ & $50 \%$ & $54 \%$ \\
\hline 2014 & $52 \%$ & $54 \%$ & $50 \%$ & $54 \%$ & $52 \%$ & $50 \%$ & $57 \%$ & $55 \%$ & $55 \%$ & $57 \%$ & $57 \%$ & $55 \%$ \\
\hline 2015 & $56 \%$ & $58 \%$ & $59 \%$ & $59 \%$ & $60 \%$ & & & & & & & \\
\hline
\end{tabular}

Table 15: Percent of PMIs apprehended by Police per month: Sept. 2014 to May 2015

\subsubsection{PERCENTAGE OF EDP FORMS COMPLETED BY POLICE PER MONTH}

Table 16 shows the percentage of EDP forms completed by police officers per month between May 2014 and May 2015. Ideally we would have begun collecting this information when we initiated our Time 1 data collection (i.e. September to December 2013). This was not possible, however, as the Police were not collecting this type of data at that time. In fact, they only began collecting this data when we pointed out the need. The first set of data available on this indicator was available in May 2014. The format of Table 16 is the same as was used in Table 15 (i.e. grey shading used to identify data that were collected after the change was introduced). While the average percentage of EDP forms completed before the implementation of any change was 59\% (i.e. May and June 2014), the average percentage of EDP forms completed after the changes were implemented was $78 \%$ (i.e. July 2014 to May 2015). The fact that the rate of completing EDP forms has increased over time (i.e. between Time 1 and Time 2), supports the idea that the strategy of mandating police officers to complete EDP forms was effective.

\begin{tabular}{|c|c|c|c|c|c|c|c|c|c|c|c|c|}
\hline & $\begin{array}{l}\text { 志 } \\
\text { 志 }\end{array}$ & 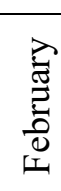 & $\begin{array}{l}\frac{5}{0} \\
\stackrel{\vec{J}}{\Sigma}\end{array}$ & $\overline{\mathrm{z}}$ & $\sum^{\mathrm{I}}$ & $\Xi$ & $\frac{\lambda}{\Xi}$ & $\begin{array}{l}\overrightarrow{w_{0}} \\
\overrightarrow{b_{0}} \\
\vec{z}\end{array}$ & 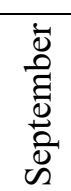 & 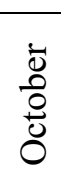 & $\begin{array}{l}\dot{0} \\
\bar{\Xi} \\
\text { ठे } \\
z\end{array}$ & 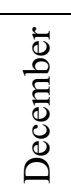 \\
\hline 2013 & & & & & & & & & & & & \\
\hline
\end{tabular}




\begin{tabular}{|l|l|l|l|l|l|l|l|l|l|l|l|l|}
2014 & & & & & $56 \%$ & $61 \%$ & $66 \%$ & $75 \%$ & $75 \%$ & $76 \%$ & $76 \%$ & $78 \%$ \\
\hline 2015 & $80 \%$ & $78 \%$ & $84 \%$ & $86 \%$ & $85 \%$ & & & & & & & \\
\hline
\end{tabular}

Table 16: Percent of EDP forms completed by Police per month: May 2014 to May 2015

\subsubsection{PERCENTAGE OF PMIS APPREHENDED BY POLICE AND FORMED BY A PHYSICIAN PER MONTH}

We calculated the percentage of PMIs apprehended by Police and formed by the ED physician per month (i.e. "apprehended - formed") by dividing the number of PMI apprehensions per month that were formed by the physician by the total number of Police apprehensions per month. These data are shown in Table 17. Similar to the format used in the two preceding tables, the gray squares identify post-change data. Examination of these data on a month by month basis show that the percentage of PMIs "apprehended-formed" has increased since the TS Planned Change was introduced from $71 \%$ on average pre change to $78 \%$, on average, post change. It is also noteworthy that, with one exception (May) the percentage "apprehended-formed" was higher each month post-change than could be observed in the same month pre-change. This analysis indicates that the proportion of PMIs apprehended by Police and formed by a physician has increased between Time 1 and Time 2, and suggests that mandating the completion of EDP forms and the mental health training had the desired effect. 


\begin{tabular}{|c|c|c|c|c|c|c|c|c|c|c|c|c|}
\hline & $\begin{array}{l}\vec{\Xi} \\
\stackrel{\vec{\Xi}}{\Xi} \\
\stackrel{\Xi}{\Xi}\end{array}$ & 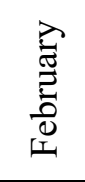 & $\begin{array}{l}\frac{1}{0} \\
\text { 䒕 }\end{array}$ & $\overline{\bar{z}}$ & $\stackrel{\vec{\Theta}}{\Sigma}$ & $\stackrel{\Xi}{\Xi}$ & $\stackrel{\gtrsim}{\Xi}$ & $\begin{array}{l}\vec{v} \\
\vec{E} \\
\vec{E} \\
\vec{Z}\end{array}$ & 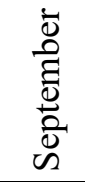 & $\begin{array}{l}\dot{\bar{D}} \\
\stackrel{0}{0} \\
\stackrel{0}{0} \\
0\end{array}$ & 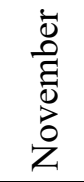 & 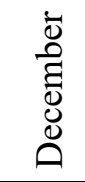 \\
\hline 2013 & & & & & & & & & $70 \%$ & $68 \%$ & $70 \%$ & $71 \%$ \\
\hline 2014 & $70 \%$ & $68 \%$ & $70 \%$ & $70 \%$ & $80 \%$ & $75 \%$ & $75 \%$ & $81 \%$ & $76 \%$ & $78 \%$ & $74 \%$ & $77 \%$ \\
\hline 2015 & $79 \%$ & $83 \%$ & $78 \%$ & $81 \%$ & $80 \%$ & & & & & & & \\
\hline
\end{tabular}

Table 17: Percent of PMIs apprehended by Police and formed by a physician: Sept 2013 to May

2015

\subsubsection{AVERAGE LENGTH OF TIME PMIS APPREHENDED BY POLICE HAVE TO WAIT TO BE ASSESSED BY A PHYSICIAN PER MONTH}

Data showing the mean number of hours PMIs apprehended by Police have to wait to be assessed by a doctor (i.e. "apprehended - wait time") are given in Table 18. Again, grey shading is used to identify data from months after the planned TS change was implemented. It should be noted that wait times can vary considerably by month as seasonal trends affect ED volumes. For example, given that the months related to cold and flu season have higher ED volumes than other months, we would expect that wait times in the winter would be longer than wait times in the summer. Accordingly, our analysis below compares monthly data on an annual basis (e.g. September 2013 data is compared to September 2014 data).

Examination of the data in Table 18 reveals that the average length of time PMIs apprehended by Police have to wait to be assessed by a physician has decreased over time (i.e. average number of hours PMIs apprehend by Police had to wait to be assessed by a physician was lower post change than reported in the same month pre-change). These data support the following conclusions: (1) the average length of time that PMIs apprehended by Police have to wait to be 
assessed by a physician decreased over time, and (2) the escalation and intervention policy had the desired effect.

\begin{tabular}{|c|c|c|c|c|c|c|c|c|c|c|c|c|}
\hline & 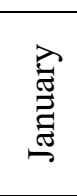 & 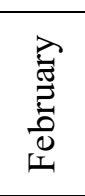 & $\begin{array}{l}\frac{\pi}{0} \\
\text { ¿̇ँ }\end{array}$ & $\overline{\bar{Z}}$ & $\stackrel{\vec{\Xi}}{\Sigma}$ & $\stackrel{\Xi}{\Xi}$ & $\vec{\Xi}$ & $\begin{array}{l}\vec{w} \\
\overrightarrow{0} \\
\vec{Z} \\
\vec{\sum}\end{array}$ & 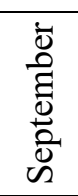 & $\begin{array}{l}\overline{0} \\
\stackrel{0}{0} \\
\stackrel{0}{0}\end{array}$ & $\begin{array}{l}\dot{\bar{D}} \\
\ddot{0} \\
\overline{0} \\
\stackrel{0}{0} \\
z\end{array}$ & 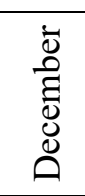 \\
\hline 2013 & & & & & & & & & 2.94 & 3.13 & 3.08 & 3.18 \\
\hline 2014 & 3.44 & 3.05 & 2.55 & 3.06 & 2.33 & 2.43 & 1.84 & 2.10 & 2.27 & 2.03 & 2.07 & 2.60 \\
\hline 2015 & 2.44 & 2.45 & 2.37 & 2.40 & 2.41 & & & & & & & \\
\hline
\end{tabular}

Table 18: Average number of hours PMIs apprehended by Police have to wait to be assessed by

a doctor: Sept 2013 to May 2015

\subsubsection{KEY FINDINGS FROM ANALYSIS OF THE CHANGE INDICATORS}

Table 19 maps the decision-makers' three objectives to our findings from the analysis of the change indicators. The data in Table 19 support the conclusion that two out of the three decision-makers' objectives were achieved over the course of this case study through the implementation of the four specific changes discussed earlier.

\begin{tabular}{|l|l|}
\hline \multicolumn{1}{|c|}{ Decision-Makers' Objectives } & \multicolumn{1}{c|}{ Key Findings } \\
\hline $\begin{array}{l}\text { Decrease the number of PMIs apprehended by } \\
\text { Police and brought to the Hospital per month }\end{array}$ & $\begin{array}{l}\text { Percentage of PMIs apprehended by Police and } \\
\text { brought to the Hospital has increased over time }\end{array}$ \\
\hline $\begin{array}{l}\text { Decrease the number of PMIs apprehended by } \\
\text { Police, brought to the Hospital, and not formed by a } \\
\text { physician per month }\end{array}$ & $\begin{array}{l}\text { Percentage of EDP forms completed by Police has } \\
\text { increased over time } \\
\text { Percentage of PMIs apprehended by Police and } \\
\text { formed by a physician has increased over time }\end{array}$ \\
\hline $\begin{array}{l}\text { Decrease the average length of time PMIs } \\
\text { apprehended by Police have to wait in the Hospital } \\
\text { before being evaluated per month }\end{array}$ & $\begin{array}{l}\text { Average length of time PMIs apprehended by } \\
\text { Police have to wait to be assessed by a physician } \\
\text { has decreased over time }\end{array}$ \\
\hline
\end{tabular}

Table 19: Decision-Makers' Objectives and Change Outcome

The first objective was to decrease the number of PMIs apprehended by police officers and brought to the Hospital per month. Analysis of the data (see Table 15) indicates that this outcome 
was not achieved. In fact, analysis of the data suggests that there has been an increase in the percentage of PMIs apprehended by police officers per month since the implementation of the planned TS change. One plausible explanation for this increase may be that the InSted program has little impact on the number of citizens with mental health issues within the community. The other possible explanation is that the additional mental health training for police officers has increased the police officers' awareness of mental health issues, and resulted in Police apprehending a higher percentage of PMIs they interact with in the community

The second objective was to decrease the number of apprehended PMIs who were brought to the Hospital by the Police and not formed by a physician. Analysis of the data found in Table 16 and Table 17 suggests that this objective has been met. The percentage of PMIs apprehended by Police and formed by a physician has increased over time. This finding may have been due to police improving their mental health training, which has allowed officers to better "diagnose" individuals with mental health issues. It may have also been affected by the fact that police officers are completing EDP forms at a higher rate, which means that police are passing along more information to physicians so that they can assess the PMIs appropriately.

The third objective was to decrease the average length of time that PMIs apprehended by Police have to wait to be assessed by a physician. Analysis of the data found in Table 18 implies that this has also occurred. The average length of time that PMIs apprehended by Police have to wait to be assessed by a physician decreased considerably from Time 1 to Time 2 . This finding would suggest that the intervention and escalation policy was effective at decreasing the length of time that PMIs apprehended by Police have to wait to be assessed by a physician. 
In conclusion, the four sets of changes (i.e. the planned TS change) that were implemented in July 2014 have had a measureable impact on three out of four of the objective change indicators used in this study. We now turn to qualitative data exploring the views of frontline employees involved in this TS with regards to what they felt needed to change and what they observed had changed in the PMI process over time.

\subsection{WHAT CHANGED: A PERCEPTUAL ANALYSIS OF THE PLANNED TS CHANGE}

This section will examine the results of data analysis relating to what respondents wanted to change within the TS at Time 1 and what they thought changed at Time 2. The analysis reported in this section is guided by the following research question:

How do TS stakeholders' perceptions of what needs to be changed with the TS compare to their perceptions of what has changed within the TS six months after the implementation of the planned TS change?

Findings presented and discussed in this section are obtained from analysis of our repeated crosssectional data set (i.e. 75 respondents at Time 1 and 60 respondents at Time 2).

The presentation of findings associated with perceptions of desired and perceived changes within the TS are broken down as follows. First, we present perceptions of what respondents felt needed to change within the TS before the implementation of planned changes. Findings using the total sample are presented first in Section 5.3.1, and then followed in Section 5.3.2 by an examination of the findings at the level of the TS stakeholder. Second, we present perceptions of what respondents felt had changed in the TS six months after the implementation of planned changes. In Section 5.3.3, we present findings from the total sample, whereas Section 5.3.4 presents an examination of these post-change findings at the level of the TS stakeholder. Lastly, 
Section 5.3.5 examines the 'sharedness' of perceptions of desired and perceived changes within the TS.

\subsubsection{PERCEPTIONS OF DESIRED CHANGES WITHIN THE TS: TOTAL SAMPLE PRE-CHANGE (TIME 1)}

Analysis of the Time 1 interviews resulted in the identification of thirteen first-order codes describing the types of changes respondents wanted to see in the TS, along with their respective second-order categories. These first-order codes and second-order categories (shaded) are displayed in Table 20. Three of these changes were not attached to any one TS stakeholder and related to changes in how the TS itself operated (i.e. transorganizational system changes). Three related to PMI process adjustments within the Hospital while three were requests for the Police to change their behaviour within the PMI process. Very few respondents talked about desired changes in how the Social Service Agency worked within the TS. Analysis of the second-order categories is reported first, followed by the analysis of first-order codes. Responses mentioned by less than $10 \%$ of the total sample will not be discussed in this section. 


\begin{tabular}{|c|c|c|}
\hline \multirow{2}{*}{$\begin{array}{l}\text { Perceptions of Desired Changes in the } \\
\text { TS: Total Sample Pre-Change (Time 1) }\end{array}$} & \multicolumn{2}{|c|}{$\begin{array}{c}\text { Total } \\
(n=75)\end{array}$} \\
\hline & \# & $\%$ \\
\hline Desired Changes at System Level & 21 & 28 \\
\hline $\begin{array}{r}\text { More effective communication between TS } \\
\text { stakeholders } \\
\end{array}$ & 16 & 22 \\
\hline Increased community supports for police & 4 & 5 \\
\hline Reduced stigmatization throughout PMI process & 4 & 5 \\
\hline Desired Hospital Changes & 59 & 79 \\
\hline Shorter waiting time for PMI and Police & 55 & 73 \\
\hline Increased MHESU engagement & 33 & 44 \\
\hline $\begin{array}{r}\text { Other Hospital Changes (i.e. improve PMI } \\
\text { experience, time to disposition, admission criteria) }\end{array}$ & 7 & 8 \\
\hline Desired Police Changes & 20 & 27 \\
\hline Training Police to apprehend PMIs appropriately & 14 & 19 \\
\hline Police compete EDP form & 6 & 8 \\
\hline $\begin{array}{r}\text { Other Police changes (i.e. communication to } \\
\text { families, response once PMI formed) }\end{array}$ & 2 & 3 \\
\hline Desired Social Service Agency Changes & 4 & 5 \\
\hline Improve Mobile Crisis services in community & 1 & 1 \\
\hline Increased psychiatric areas in the community & 1 & 1 \\
\hline $\begin{array}{r}\text { Increased Social Service Agency training to improve } \\
\text { initial assessments }\end{array}$ & 1 & 1 \\
\hline More mental health education for family & 1 & 1 \\
\hline
\end{tabular}

Table 20: Perceptions of Desired Changes in the TS: Total Sample Pre-Change (Time 1)

Second-Order Categories: Our analysis of the second-order categories shows that: respondents were more than twice as likely to mention desired changes within the Hospital (79\% of total sample) than to mention desired changes at either the system level ( $28 \%$ of total sample) or within the Police ( $27 \%$ of total sample). Virtually none of the respondents ( $5 \%$ of total sample) expressed a desire for a change to the Social Service Agency. Analysis of the first-order codes helps us understand why this might be the case. 
Desired Changes at System Level: Respondents mentioned three changes that they wanted to see within the TS that were not directly attached to any specific TS stakeholder. Only one of these responses was mentioned by more than $10 \%$ of the sample. In this case, one in five respondents talked about the lack of communication or miscommunication between TS stakeholders within the current PMI process and identified ways that they would like to see communication be improved within the PMI process. This type of comment was given the descriptor 'more effective communication between TS stakeholders'. For example:

"I think there could be clearer communication with... when do you stay, when do you go... because a lot of the nurses might not know that the Police can leave, or we ask them if they want to leave and it may not be really clear communication. I think that's something that could be improved." (Hospital MHESU Psychiatrist)

"Communication could be a lot better between the Hospital and Police. Building that relationship needs to happen. I know sometimes you look on the nurses' and doctors' faces... they're not too happy that we're there. There have been occasions where they're telling us what we should have done out on the road, and that shouldn't be happening." (Police Sergeant)

"I think the communication between the Hospital and the [Social Service] organisation, something happens along the way, kind of like a broken telephone. I know that when we send our information that we highlight it at the time is a part of the sort of community treatment team, but for some reason things just get missed, they'll miss medication that they're on, even though we send them our contact information they'll say they tried to contact us and they don't get our information, they don't get us on the phone, so therefore they 
couldn't inject the client with the injection, they couldn't treat, so they discharged them." (Social Service Agency Frontline)

Desired Hospital Changes: During the Time 1 interviews, respondents identified two changes that they would like the Hospital to make with respect to the PMI process: (1) shorten waiting time for Police, and (2) increase MHESU engagement. In the first case, seventy-three percent of the respondents interviewed at Time 1 mentioned that they thought PMIs and police officers were waiting too long in the Hospital, and wanted the Hospital to make changes in order to reduce the amount of time PMIs and Police were waiting. This type of comment was given the descriptor 'shorten waiting time for PMI and Police'. For example:

"I think we're pretty timely in getting our police officers out, but I think that's something that we could improve upon." (Hospital Manager)

"I believe it's the wait time between the individual arriving [at the ED] and getting assessed by the ER [emergency room] doctor. Yes, since we're so busy, one of the busiest hospitals in Ontario.... it takes a while for the individual to be seen by the ER doctor and assessed." (Hospital Nurse)

"The waiting times there are absolutely unacceptable. You have... you have two officers that can be out in the road increasing public safety, increasing officer safety, increasing all kinds of things, meanwhile, we're stuck sitting at the hospital and it's not just one or two people that get arrested daily for Mental Health Act, it's three, four, five, six, seven, it's several people daily that are arrested under the Mental Health Act so we have multiple officers tied up. I've seen where we've had as many as fifteen cruisers lined up out in front of emerge 
sitting there because we have Mental Health Act patients that need to be seen but just aren't getting taken care of. The Hospital needs to address this." (Police Sergeant)

"I have spent up to eight hours in emergency with a suicidal client before even being seen by a physician; that's not right." (Social Service Agency Frontline)

Respondents also commented that employees within the MHESU (e.g., crisis workers, social workers, mental health nurses, and psychiatrists) were either not involved early enough in the PMI process or needed to be involved more regularly in the PMI process. Nearly half of the respondents interviewed at Time 1 made this type of comment, which we labeled: 'Increased MHESU engagement' to reflect the change they desired. The following quotes illustrate this firstorder code:

"The social worker is the filter because the [ED] doctor doesn't want to go in and sit for 45 minutes to an hour and talk with the patient and hear every single detail. They don't have the time to do that because you could have 20 mental health patients at any one time to sit down with. The problem is the social worker doesn't want to engage with the patient unless they have an $[E D]$ doctor to either form or help the social worker manage or guide that patient through after." (Hospital Resource Nurse)

"I mean mental health patients don't get ill 9-5 Monday to Friday and yet for whatever reason we don't have our psychiatry team available 24/7 and so patients get backlogged late at night and weekends, unsociable hours. Unlike other services like surgery and internal medicine, psychiatry continues to work pretty cushy hours. Now they have made improvements in that they now take 9-10 pm on most weekdays but part of the flow process would be a collaborative approach would be having a psychiatrist not necessarily 24/7 but 
available more quickly to do their assessments in conjunction with the emergency physician who is worried about the medical stability of the patient because you have way too much handover... right now you have the police telling the ER telling the social worker telling the psychiatrist what is going on, whereas if the police were communicating directly with the psychiatrist or at least the social worker there would be less steps where information could get lost." (Hospital ED Physician)

"I know that they have a process in the Hospital to be medically cleared first, so sometimes psychiatrists may be available, but they won't see the client until the medical doctor will have seen the client first." (Social Service Agency Frontline)

Desired Police Changes: Approximately a quarter of the respondents in the Time 1 sample mentioned that they would like to see a change to how the Police operated within the PMI process. The most frequently mentioned desired change to Police's operations related to their mental health training program. Fourteen out of the seventy-five employees interviewed before the implementation of changes mentioned that they thought more mental health training would improve how Police apprehended PMIs. We labeled this type of comment: 'Training Police to apprehend PMIs appropriately'. Illustrative quotes for this first-order code are provided below:

"I think the times when it [the PMI process] doesn't work very well are because the front line people that are going out, which happen to be police officers, aren't necessarily trained well enough in the area of intervention within mental health, and I think that's the sort of major change that needs to happen." (Social Service Agency Frontline)

“...Police education... I guess sometimes the language that they use, the way that they identify individuals. They tend to flag individuals as EDP and that's what they refer to them 
as, the EDP and not by their names or last names or what not. So yeah education, the way they deal with the clients, the way that they speak to them, things like that... Sometimes they can be quite forceful and maybe handcuffs are not necessary in some situations and maybe they are necessary in some situations but I think if they were educated they would understand what is dangerous and what's not." (Social Service Agency Frontline)

Desired Social Services Changes: Finally, very few respondents gave suggestions on how the Social Service Agency could change to improve the PMI process.

\subsubsection{PERCEPTIONS OF DESIRED CHANGES WITHIN THE TS: TS STAKEHOLDER SAMPLE PRE-CHANGE (TIME 1)}

Table 21 provides similar information to that which was shown in Table 20, but in this case, the data are broken down by TS stakeholder to allow us to determine similarities and differences in what the various TS stakeholders wanted to change before the implementation of the planned TS change. Analysis of the second-order categories is reported first, followed by the analysis of first-order codes. 


\begin{tabular}{|c|c|c|c|c|c|c|c|}
\hline \multirow{2}{*}{$\begin{array}{c}\text { Perceptions of Desired Changes in the } \\
\text { TS: TS Stakeholder Sample Pre-Change } \\
\text { (Time 1) }\end{array}$} & \multicolumn{2}{|c|}{$\begin{array}{c}\text { Hospital } \\
(n=25)\end{array}$} & \multicolumn{2}{|c|}{$\begin{array}{l}\text { Police } \\
(n=25)\end{array}$} & \multicolumn{2}{|c|}{$\begin{array}{l}\text { Social Service } \\
\text { Agency } \\
(\mathbf{n}=\mathbf{2 5})\end{array}$} & \multirow[t]{2}{*}{$\begin{array}{c}\text { Perception } \\
\text { (Common / Shared / } \\
\text { Unique) }\end{array}$} \\
\hline & \# & $\%$ & \# & $\%$ & \# & $\%$ & \\
\hline Desired Changes at System Level & 4 & 16 & 7 & 28 & 13 & 52 & Strong Unique (SS) \\
\hline $\begin{array}{r}\text { More effective communication between TS } \\
\text { stakeholders }\end{array}$ & 1 & 4 & 7 & 28 & 8 & 32 & Weak Shared (P, SS) \\
\hline Desired Hospital Changes & 23 & 92 & 25 & 100 & 11 & 40 & Strong Shared $(\mathrm{H}, \mathrm{P})$ \\
\hline Shorter waiting time for PMI and Police & 20 & 80 & 25 & 100 & 10 & 40 & Strong Shared $(\mathrm{H}, \mathrm{P})$ \\
\hline Increased MHESU engagement & 21 & 84 & 7 & 28 & 5 & 20 & Strong Unique $(\mathrm{H})$ \\
\hline Desired Police Changes & 10 & 40 & $\mathbf{0}$ & $\mathbf{0}$ & 12 & 48 & Weak Shared (H, SS) \\
\hline Training Police to apprehend PMIs appropriately & 4 & 16 & 0 & 0 & 12 & 48 & Weak Unique (SS) \\
\hline Police complete EDP form & 6 & 24 & 0 & 0 & 0 & 0 & Weak Unique $(\mathrm{H})$ \\
\hline
\end{tabular}

Note: $\mathrm{H}=$ Hospital; $\mathrm{P}=$ Police; $\mathrm{SS}=$ Social Service Agency. Only responses mentioned by at least three respondents (i.e. $>10 \%$ ) from a TS stakeholder are shown.

Table 21: Perceptions of Desired Changes in the TS: TS Stakeholder Sample Pre-Change (Time 1) 
None of the second-order categories met our criteria for a common perception. Two of the second-order categories were classified as shared perceptions. More specifically, respondents from the Hospital and the Police samples strongly shared a similar perception that they desired changes within the Hospital. Similarly, respondents from the Hospital and the Social Service samples weakly shared a similar perception that they desired changes within the Police. Lastly, we identified one strong perception held uniquely by Social Service Agency respondents. More specifically, half of the respondents in the Social Service Agency sample desired a change at the TS level.

Additional information can be obtained by looking at the first order codes. The finding that, despite the fact there was a high number of desired changes identified at Time 1, there were no commonly shared perceptions between the various groups on what needed to change within the TS (i.e. no "desired changes" first-order code met our criteria for a common perception). This finding indicates that there was no consensus within the TS on how the PMI process should change. That being said, our analysis of the data identified two shared and three unique perceptions of how the system should change that are worthy of note.

Consideration of the two shared perceptions of desired changes within the TS reveals that one of them was a strong view while the other was a weak view. More specifically, the majority of police officers ( $100 \%$ of Police sample) and healthcare workers ( $80 \%$ of Hospital sample) strongly shared the perception that PMI and Police wait times needed to decrease, while one in three respondents in the Police (28\% of Police sample) and the Social Service Agency (32\% of Social Service Agency sample) samples mentioned that communication needed to improve between TS stakeholders (i.e. weak shared perception). These findings would suggest that 
respondents are more aware of the need to make changes associated with decreasing PMI and Police wait times than for improving communication between TS partners.

Lastly, our analysis of the data revealed three unique perceptions of desired changes within the TS. In the first case, a majority of healthcare workers ( $84 \%$ of Hospital sample) wanted to see increased engagement from the MHESU in the PMI process. This perception was strongly held by this group. In the second case, respondents in the Social Service Agency (32\%) weakly held the unique view that Police needed better training to improve how they apprehend PMIs who are having a mental health crisis. In the third case, twenty-four percent of those in the Hospital sample held the unique perception that Police did not complete EDP forms consistently when bringing PMIs to the Hospital. This perception met our classification of a weak desired change. We labeled this type of comment 'Police complete EDP form'. For example, two healthcare workers commented:

"The EDP form is rarely done. So there's inconsistent completion of the EDP form by Police.

So the communication of what had happened in the community to the Hospital is... I mean there's some communication but I think that could be improved." (Hospital MHESU Manager)

“A number of police forces in the province, use what's called an EDP form, an Emotionally Disturbed Person form. And in [another hospital] I work at, I get a copy of it every single time. There is never a patient who makes it to psychiatry without that form being on their chart. This is something that I believe [the Police] are supposed to fill out. I have yet to see one in the three months that I have been there. So, and I've seen a fair number of patients brought in by Police. But for whatever reason, a copy of it doesn't seem to make it onto the 
patient's chart. And so, if they do fill out a form, it's been entirely useless to me because I don't see it." (Hospital MHESU Psychiatrist)

The following points summarize our analysis of what respondents wanted to change within the TS before the implementation of the planned TS change, using the cross-sectional data set:

- Police and Hospital stakeholders strongly shared the view that changes were needed to shorten the length of time police officers were waiting at the Hospital for their PMI to be assessed by a physician.

- Police and Social Service Agency stakeholders weakly shared the view that there should be more communication between TS stakeholders.

- Hospital stakeholder expressed a strong desire to increase MHESU engagement, and a weak desire to have police officers complete EDP forms.

- Social Service Agency expressed a weak desire for police to receive more mental health training.

\subsubsection{PERCEPTIONS OF WHAT HAS CHANGED IN THE TS: TOTAL SAMPLE POST-} CHANGE (TIME 2)

Analysis of the Time 2 interview data resulted in the identification of nine first-order codes which described the ways in which the respondents perceived the TS had changed since the implementation of the planned TS change. All first-order codes are grouped with their respective second-order category and displayed in Table 22. Two of these changes were not attached to any one TS stakeholder and related to changes in how the TS itself operated (i.e. grouped under the second-order category: perceived system level changes). Two were related to PMI process 
adjustments within the Hospital, two were changes to how the Police behaved within the TS, and two were associated with changes to how the Social Service Agency operated in the PMI process. Analysis of the second-order categories is presented first. This is followed by a discussion of the first-order codes. Three of the first-order codes were mentioned by less than $10 \%$ of the total sample and will not be discussed in this section.

\begin{tabular}{|c|c|c|}
\hline \multirow{2}{*}{$\begin{array}{l}\text { Perceptions of What Has Changed in the } \\
\text { TS: Total Sample Post-Change (Time 2) }\end{array}$} & \multicolumn{2}{|c|}{$\begin{array}{c}\text { Total } \\
(\mathbf{n}=60)\end{array}$} \\
\hline & \# & $\%$ \\
\hline Perceived System Level Changes & 37 & 62 \\
\hline Increased interorganizational communication & 37 & 62 \\
\hline Greater understanding of TS stakeholder roles & 4 & 7 \\
\hline Perceived Hospital Changes & 36 & 60 \\
\hline $\begin{array}{r}\text { ED created new process that expedites service for } \\
\text { PMIs who are brought in by Police }\end{array}$ & 35 & 58 \\
\hline $\begin{array}{r}\text { Hospital increased communication between ED and } \\
\text { MHESU }\end{array}$ & 9 & 15 \\
\hline Perceived Police Changes & 17 & 28 \\
\hline Police mandate completion of EDP form & 14 & 23 \\
\hline $\begin{array}{r}\text { Police increased communication between frontline } \\
\text { officers and supervisors }\end{array}$ & 6 & 10 \\
\hline Perceived Social Service Agency Changes & 21 & 35 \\
\hline $\begin{array}{r}\text { Social Service Agency created InSted program to } \\
\text { support PMI }\end{array}$ & 21 & 35 \\
\hline $\begin{array}{r}\text { Social Service Agency changed how they make } \\
\text { decisions (i.e. risk tables) }\end{array}$ & 1 & 2 \\
\hline Perceived No Change within PMI Process & 5 & 8 \\
\hline Perceived no change within PMI process & 5 & 8 \\
\hline
\end{tabular}

Table 22: Perceptions of What Has Changed in the TS: Total Sample Post-Change (Time 2)

Second-order categories: Several observations can be made by examining the data associated with the second-order categories (shaded in Table 22). First, it is interesting to note that when asked during the Time 2 interviews what changes they perceived had occurred within the TS, respondents were twice as likely to say that they observed system level changes (62\% of 
total sample) or Hospital changes (60\% of total sample) than to talk about Police changes ( $28 \%$ of total sample) or Social Service Agency changes (35\% of total sample). Second, the fact that only five respondents ( $8 \%$ of total sample) were not able to identify a single change that had occurred within the TS between Time 1 and Time 2 suggests that the four sets of changes that were initiated as part of the planned TS change did, in fact, impact the PMI process in an observable fashion. Analysis of the first-order codes helps us understand why this might be the case.

Perceived System Level Changes: According to almost two-thirds of the employees who participated in the Time 2 interviews, communication TS stakeholders had increased within the TS over time. The following quotes illustrate the meaning of the first-order code 'increased interorganizational communication':

"I would just say increased communication between the Police and primarily myself in the Hospital about who's coming in and what type of patients they're bringing in and how long they've been waiting. " (Hospital Resource Nurse)

"A lot of the time, prior to this year, before these initiatives, we would typically be sitting in a back hall, sometimes we may not be seen, or we may be seen initially, triaged, and then put somewhere and forgotten about, and then when things become available later on down the road, we could spend two, three, four, five hours in the Hospital, sometimes up to eight hours in the Hospital, waiting for a room or waiting to see a doctor. Now, typically, if we go to the Hospital, we have a more positive interaction with the staff, and typically we'll be seen quicker or at least our concerns will be addressed. If there's no room, at least somebody's telling us, here's the situation." (Police Constable) 
"Yes, we're supposed to contact our supervisor and the supervisor comes and he speaks to, I guess, the charge nurse in regards to having the doctor come out and see us." (Police Constable)

"I've noticed that, in the past, we wouldn't really get any calls from the Hospital, and it would be like the time would go by... but I think that they're getting a bit better with calling us faster. Thinking about that, when the person does get admitted they are now calling us within a day or two ... yes, that's certainly got better." (Social Service Agency Frontline)

Perceived Hospital Changes: Over half of the respondents interviewed $(58 \%$ of total sample) mentioned that they had observed improvements to the flow of PMIs within the ED since the changes had been implemented which they attributed to their impression that healthcare workers expedited the assessment process for PMIs brought in by Police. Many of these respondents, when talking about this change, specifically mentioned the Hospital's two-hour goal of assessing PMIs brought in by police officers. The descriptor 'ED created a new process that expedites service for PMIs who are brought in by Police' was given to this type of comment. The following quotes illustrate this perceived changed:

"Well the changes are that when the police officers bring the patients to the hospital we have to see them within two hours, despite the acuteness of other patients that are in the department or the high volumes in the department. So we have to attend to these patients in the same two hours." (Hospital ED Nurse)

"I think we've got a better way of identifying that this patient population is in the emerge and escalating a process when we're reaching that target point of two hours." (Hospital Manager) 
"Now there's a triage nurse available for us to liaise with to ensure that if our officers are beyond the two-hour time limit that they, I guess for lack of a better term, be pushed up as far as priority is concerned so that has certainly helped things a lot." (Police Sergeant)

Nine respondents $(15 \%$ of the total sample) noted during the Time 2 interviews that communication between the ED and the MHESU had increased, an occurrence they attributed to the fact that the ED expedites assessments for PMIs who are brought in by Police. This type of comment was given the label: 'Hospital increased communication between ED and MHESU'. Illustrative quotes describing this first-order code are provided by Hospital employees below:

"I think we talk more with the ED folks because... there's more people being formed from there, so you're having this discussion of policies and procedures of the hospital more often." (Hospital Security Guard)

"I would say there is a bit more communication than there was before. Because the numbers are high they need to be on point with security, we all need to be on the same page or else something bad is going to happen." (Hospital ED Nurse)

"I think it [decreased wait times] has forced the emergency to have better communication with psychiatrists on a more timely basis... What's happening up to the minute in the Emerg in regards to psychiatric patients to be assessed by psychiatry, as opposed to a year ago they [PMIs] were waiting probably 24 hours in the Emerg for a psychiatric assessment and I would say, now, it's improved quite a bit, I would say sometimes it's relatively soon after or up to eight to 12 hours after, so, probably improved 50\%, this last year." (Hospital Resource Nurse) 
Perceived Police Changes: Respondents interviewed after changes were implemented also identified two ways in which they felt that Police behaviour had changed after the introduction of the planned TS change: (1) Police mandate completion of EDP form, and (2) increased communication between frontline officers and their supervisors. About a quarter of the total sample mentioned that, as a result of the implemented changes, Police were mandated to document the PMI process with an EDP form. This type of comment was given the descriptor 'Police mandate completion of EDP form'. Sample comments that were given this code label are provided below.

"I believe last year when we spoke Police weren't filling out those EDP forms and now... It looks like we're really consistent on having those things filled out and kept and stuff like that." (Hospital ED Nurse)

"It's filled out at the Hospital and brought back to Police. Yes, it seems to me officers now are aware that they have to be filling out that form and submitting it, whereas before maybe it wasn't as clear as to what they were supposed to do with it, but the importance of it now is much more important." (Police Sergeant)

"There has been changes in our service as to how we do those [EDP forms]. Before, I think it was more of a, more voluntary, I guess? Just if the officer felt like filling it out, and it's now been made mandatory as part of our service, to fill it out and send it to the mental health coordinator." (Police Constable)

Ten percent of respondents interviewed after changes were implemented within the TS mentioned that communication between frontline police officers and their supervisors had increased over the past year. These individuals attributed this increase to the introduction of new 
procedures associated with filling out EDP forms (i.e. frontline officers had to meet with their supervisors so that their supervisor could sign off on the EDP form). This type of comment was given the label 'Police increased communication between frontline officers and supervisors'. For example:

"I realize the [EDP] forms that they fill out now more or less are for tracking purposes. I would assume allowing our organization to have a better understanding as far as what the wait times are... But Imean as far as the greater picture it's forcing us to communicate more with our staff and be more cognizant of the wait times." (Police Sergeant)

"It has given us time to chat and review the outcome because we're checking out - did he end up getting formed? How long were you there? That sort of thing, so a little bit more debriefing the process and things after the fact." (Police Sergeant)

Perceived Social Service Agency Changes: Finally, when asked what changes they observed to the PMI process at Time 2, respondents mentioned one change related to the Social Service Agency's role in the PMI process. More specifically, over a third of the respondents ( $35 \%$ of total sample) mentioned the In-Short-Term-Emergency-Deviation (InSted) Program or aspects of this program. These respondents discussed how the InSted program that had been introduced involved a Social Service employee working from within the MHESU to coordinate the discharge of PMIs from the Hospital to community services. The following comments are examples of quotes that were given the descriptor 'Social Service created InSted program to support PMI':

"Within the last year we have within the Social Service initiated a programme called in short term emergency diversion which is the InSted programme." (Social Service Agency Manager) 
"We started up a brand new programme. We're seeing clients who otherwise would not be served quickly, because there's no other programme in [the city] that can see people that quickly and consistently during crisis. So we've seen clients go from Hospital to InSted to our short term case managers within... pretty much immediately. They'd be seen, intaked and then seen by a worker following their discharge from hospital. So there's no other program that does that.” (Social Service Agency Frontline)

"MHESU doesn't have a discharge planner on the unit, and so, when people were being discharged before, it was a matter of giving phone numbers and hoping that that individual would then follow up on those numbers. Now, we can provide that telephone follow-up... And we can refer to my counterparts in the community. So I guess we're bridging that gap, in the fact that we can provide that telephone follow-up." (Social Service InSted Worker)

\subsubsection{PERCEPTIONS OF WHAT HAS CHANGED IN THE TS: TS STAKEHOLDER SAMPLE POST-CHANGE (TIME 2)}

Table 23 shows six first-order codes and four second-order categories representing what respondents had observed had changed within the TS after the implementation of the planned TS change. These data are broken down by TS stakeholder. We first report our analysis of secondorder categories (shaded), then present our analysis of first-order codes. 


\begin{tabular}{|c|c|c|c|c|c|c|c|}
\hline \multirow{2}{*}{$\begin{array}{l}\text { Perceptions of What Has Changed in the } \\
\text { TS: TS Stakeholder Sample Post-Change } \\
\text { (Time 2) }\end{array}$} & \multicolumn{2}{|c|}{$\begin{array}{l}\text { Hospital } \\
(n=20)\end{array}$} & \multicolumn{2}{|c|}{$\begin{array}{l}\text { Police } \\
(n=20)\end{array}$} & \multicolumn{2}{|c|}{$\begin{array}{l}\text { Social Service } \\
\text { Agency } \\
(\mathbf{n}=\mathbf{2 0})\end{array}$} & \multirow{2}{*}{$\begin{array}{c}\text { Perception } \\
\text { (Common / Shared / } \\
\text { Unique) }\end{array}$} \\
\hline & $\#$ & $\%$ & $\#$ & $\%$ & $\#$ & $\%$ & \\
\hline Perceived System Level Changes & 12 & 60 & 13 & 65 & 12 & 60 & Strong Common \\
\hline Increased interorganizational communication & 12 & 60 & 13 & 65 & 12 & 60 & Strong Common \\
\hline Perceived Hospital Changes & 18 & 90 & 17 & 85 & $\mathbf{0}$ & $\mathbf{0}$ & Strong Shared $(\mathrm{H}, \mathrm{P})$ \\
\hline $\begin{array}{r}\text { ED created new process that expedites service for } \\
\text { PMIs who are brought in by Police }\end{array}$ & 18 & 90 & 17 & 85 & 0 & 0 & Strong Shared $(\mathrm{H}, \mathrm{P})$ \\
\hline $\begin{array}{r}\text { Hospital increased communication between ED and } \\
\text { MHESU }\end{array}$ & 8 & 40 & 1 & 5 & 0 & 0 & Strong Unique $(\mathrm{H})$ \\
\hline Perceived Police Changes & 1 & 5 & 14 & 70 & $\mathbf{0}$ & $\mathbf{0}$ & Strong Unique $(\mathrm{P})$ \\
\hline Police mandate completion of EDP form & 1 & 5 & 13 & 65 & 0 & 0 & Strong Unique $(\mathrm{P})$ \\
\hline $\begin{array}{r}\text { Police increased communication between frontline } \\
\text { officers and supervisors }\end{array}$ & 0 & 0 & 6 & 30 & 0 & 0 & Weak Unique (P) \\
\hline Perceived Social Service Agency Changes & 5 & 25 & $\mathbf{0}$ & $\mathbf{0}$ & 16 & 80 & Strong Unique (SS) \\
\hline $\begin{array}{r}\begin{array}{r}\text { Social Service Agency created InSted program to } \\
\text { support PMI }\end{array} \\
\end{array}$ & 5 & 25 & 0 & 0 & 16 & 80 & Strong Unique (SS) \\
\hline
\end{tabular}

Note: $\mathrm{H}=$ Hospital; $\mathrm{P}=$ Police; $\mathrm{SS}=$ Social Service Agency.

Table 23: Perceptions of What Has Changed in the TS: TS Stakeholder Sample Post-Change (Time 2) 
Our analysis identified four second-order categories describing what respondents perceived had changed in the TS post the introduction of the planned TS change. In the first case, there was a strong common perception of change at the system level. In the second instance, there was a strong shared perception between the Hospital and Police of a change within the Hospital. In the last two cases, there were strong unique perceptions that the Police and Social Service Agency perceived changes within their TS stakeholder.

Examination of the data associated with the six first-order codes provides additional information on how the different TS stakeholders perceived that the planned TS change had impacted the TS. Our analysis showed that two-thirds of the respondents in each TS stakeholder sample perceived that communication between TS stakeholders had increased and improved over time. This meets the criteria for classification as a strong common perception.

Additionally, a second first-order code met our classification for a shared perception. In this case, the majority of respondents in the Hospital ( $90 \%$ of Hospital sample) and Police (85\% of Police sample) samples mentioned that healthcare staff were now expediting service for PMIs who were brought in by the Police.

Finally, three perceptions of how the TS had changed over time were held uniquely by one of the three TS stakeholders. Community support workers ( $80 \%$ of Social Service sample) strongly held the unique perception that the introduction of the InSted program was a change in the TS. Respondents in the Police sample, on the other hand, held two unique perceptions. More specifically, Police informants uniquely perceived that they had changed when they were mandated to formally complete the EDP form ( $65 \%$ of Police sample) and that there was now more frequent communication between frontline officers and their supervisors ( $30 \%$ of Police sample). 
Summarizing our analysis of what respondents observed had changed within the TS after the implementation of the planned TS change is the following list of five points:

- All three TS stakeholders strongly shared the common view that there was increased communication between TS partners post-change.

- Hospital and Police stakeholders strongly shared the view that the planned TS change resulted in a new ED process to expedite medical service for PMIs who were brought in by police officers.

- The Hospital expressed the strong view that there was more communication between the ED and the MHESU post-change as compared to pre-change.

- Police had the view that the planned TS change had impacted their protocol of filling out the EDP form (strong), and their communication between frontline officers and sergeants (weak) post-change.

- Respondents in the Social Service Agency sample had the strong view that the InSted program started post-change.

\subsubsection{PERCEPTIONS OF DESIRED AND PERCEIVED CHANGES WITHIN THE TS:} ANALYSIS OF THE CROSS-SECTIONAL DATA SET

The purpose of this section is to examine the cross-section data set in order to increase our understanding of: (1) the extent to which the changes the various TS stakeholders perceived had been implemented within the system corresponded to changes that they had indicated that they wanted to see implemented, and (2) how these perceptions might potentially impact TS stakeholder relationships. To do so, we followed three steps. 
First, we examined Time 1 data describing the changes that the respondents in the three stakeholder groups wanted to see in the TS. We classified responses (first-order codes and secondorder categories) into three categories: (1) common (all three stakeholders wanted these changes to the TS), (2) shared (two out of three stakeholders wanted these changes to the TS), and (3) unique (only one stakeholder wanted this change to the TS). We also categorized these perceptions as being strong $(50 \%$ or more of respondents in each relevant stakeholder group wanted this change) or weak (less than $50 \%$ of the respondents in each relevant stakeholder group wanted this change).

Second, we examined Time 2 data regarding the changes that the respondents in the three stakeholder groups observed in the TS six months after the planned change had been implemented. We again classified responses (first-order codes and second-order categories) into three categories: (1) common, (2) shared, and (3) unique. We also categorized these perceptions as being strong or weak as noted above.

Third, we examined this categorization of our data and looked for evidence of shifts in 'sharedness' over time (see Table 24). We operationally define a shift in 'sharedness' by comparing the classification we gave a perception of desired changes in the TS at Time 1 (e.g. strong common) with the classification we gave the corresponding perception of observed changes in the TS at Time 2 (e.g. weak shared). We identify three different scenarios (see Table 24) using this analysis: (1) Got Desired Change (i.e. TS stakeholders perceived a change that they desired), (2) Thwarted Desires (i.e. more TS stakeholders desired a change in the TS than those who observed that particular change had occurred), and (3) Unanticipated Change (i.e. fewer TS 
stakeholders desired a change in the TS than those who observed that particular change had occurred). In the following section, we present data supporting each of these three scenarios.

Got Desired Change. Our analysis supports the idea that, in one case, two partners (i.e. Hospital and Police) felt that they got a change that they wanted. Cases where the TS stakeholders felt that a "desired change" had occurred are listed below.

- $\quad$ Pre-change both the Hospital and Police desired changes in how the Hospital operated within the TS (strong shared) (i.e. Desired/Perceived Hospital Changes). Post-change both the Police and the Hospital noted that such a change had occurred (strong shared).

- $\quad$ Pre-change both the Hospital and the Police wanted the Hospital to expedite service for PMIs who were brought in by the Police (strong shared) (i.e. New process that expedites service for PMIs who are brought in by Police). Similarly, post-change both the Hospital and the Police mentioned that such a change had occurred (strong shared)

- $\quad$ Before the implementation of the planned TS change, the Hospital wanted changes within their own organization which would result in increased MHESU engagement in the TS process (strong unique) (i.e. Increased MHESU engagement). A portion of healthcare workers noted that, post-change, such a change had occurred (weak unique).

The above data support the idea that the planned TS change provided the Hospital and Police with a change that they wanted pre-change. 


\begin{tabular}{|c|c|c|c|}
\hline $\begin{array}{c}\text { Perceptions of Desired (Time 1) and Perceived } \\
\text { (Time 2) Changes in the TS }\end{array}$ & $\begin{array}{c}\text { Pre-Change } \\
\text { Perception (Common } \\
\text { / Shared / Unique) }\end{array}$ & $\begin{array}{c}\text { Post -Change } \\
\text { Perception } \\
\text { (Common / Shared } \\
\text { / Unique) }\end{array}$ & $\begin{array}{l}\text { Shift in } \\
\text { 'Sharedness' }\end{array}$ \\
\hline Desired/Perceived Changes at System Level & Strong Unique (SS) & Strong Common & Unanticipated Change \\
\hline More effective communication between TS stakeholders & Weak Shared (P, SS) & Strong Common & Unanticipated Change \\
\hline Desired/Perceived Hospital Changes & Strong Shared $(\mathrm{H}, \mathrm{P})$ & Strong Shared $(\mathrm{H}, \mathrm{P})$ & Got Desired Change \\
\hline $\begin{array}{r}\text { New process that expedites service for PMIs who are } \\
\text { brought in by Police }\end{array}$ & Strong Shared $(\mathrm{H}, \mathrm{P})$ & Strong Shared $(\mathrm{H}, \mathrm{P})$ & Got Desired Change \\
\hline $\begin{array}{r}\text { Increased MHESU engagement (i.e. Hospital increased } \\
\text { communication between ED and MHESU) }\end{array}$ & Strong Unique $(\mathrm{H})$ & Weak Unique $(\mathrm{H})$ & Got Desired Change \\
\hline Desired/Perceived Police Changes & Weak Shared (H, SS) & Strong Unique (P) & $\begin{array}{c}\text { Thwarted Desires/ } \\
\text { Unanticipated Change }\end{array}$ \\
\hline Training Police to apprehend PMIs appropriately & Weak Unique (SS) & & Thwarted Desires \\
\hline Police compete EDP form & Weak Unique $(\mathrm{H})$ & Strong Unique (P) & $\begin{array}{c}\text { Thwarted Desires/ } \\
\text { Unanticipated Change }\end{array}$ \\
\hline $\begin{array}{r}\text { Police increased communication between frontline officers } \\
\text { and supervisors }\end{array}$ & & Weak Unique (P) & Unanticipated Change \\
\hline Desired/Perceived Social Service Agency Changes & & Strong Unique (SS) & Unanticipated Change \\
\hline $\begin{array}{r}\text { Social Service Agency created InSted program to support } \\
\text { PMI }\end{array}$ & & Strong Unique (SS) & Unanticipated Change \\
\hline
\end{tabular}

Note. Only responses associated with first-order codes that were given a common, shared, or unique label at Time 1 and/or at Time 2 are shown.

Table 24: Comparison of Common, Shared, and Unique Perceptions of Desired (Time 1) and Perceived (Time 2) Changes in the TS 
Thwarted Desires: Findings indicate that two TS stakeholders (i.e., Hospital and Social Service Agency) desired a change within the Police that they did not observe six-months post change. Occasions where the TS stakeholders desire for a particular change were "Thwarted" are listed below.

- Pre-change both the Hospital and Social Service Agency desired changes in how the Police operated within the TS (weak shared) (Desired/Perceived Police Changes). After the introduction of the planned TS change, however, only the Police felt that such a change had occurred (strong unique).

- In Time 1 interviews, the Social Service Agency indicated that they wanted the Police to receive training in how to apprehend PMIs more appropriately (weak unique) (Training Police to apprehend PMIs appropriately). Unfortunately, the respondents in the Social Service Agency did not observe that such a change had occurred post-change.

- In Time 1 interviews the Hospital indicated that they wanted the Police to fill out the EDP form (weak unique Hospital) (Police complete EDP form). At Time 2, however, only respondents from the Police observed such a change had occurred (strong unique Police).

In this case, data shows that neither the Hospital nor the Social Service Agency observed that the Police had changed their behavior in the manner that they desired. More specifically, although before the implementation of any changes the Social Service Agency expressed the desire for the Police to receive more training on how to apprehend PMIs and the Hospital wanted the Police to fill out their EDP form, neither group reported that they had observed these changes six months after the implementation of the planned TS change. These findings are particular interesting given that the objective data does show these changes were in fact implemented. 
Unanticipated Change: Our analysis identified three situations where TS stakeholders observed a change in the TS that they had not mentioned they desired. In the first scenario, the Hospital did not mention they desired changes to interorganizational communication pre-change but, after the changes were implemented, they had greater interorganizational communication.

- Before the introduction of the planned TS change, only the Social Service Agency mentioned that they wanted a system level change within the TS (strong unique Social Service Agency) (Desired/Perceived Changes at System Level). Post-change, all stakeholders felt that such a change had occurred (strong common).

- In Time 1 data, the Police and the Social Service Agency mentioned that they wanted more effective communication between TS stakeholders (weak shared Police and Social Service Agency) (More effective communication between TS stakeholders). At Time 2, all stakeholders felt that such a change had occurred (strong common).

These findings suggest that the Hospital observed more effective communication between TS stakeholders at Time 2, despite not mentioning that they wanted it in the Time 1 interviews. It would also appear that, even though the Police wanted more effective communication between partners, they did not see the need for changes at the system level as much as the Social Service Agency at Time 1.

Second, examination of the data indicate that the Police observed an Unanticipated Change within their own organization. For example:

- Before the implementation of the planned TS change, the Police did not express any desire to change their behavior within the TS (Desired/Perceived Police Changes). After the planned TS change was introduced, however, the Police felt that they had changed their behavior in the TS in two ways (strong unique Police). 
- In Time 1 interviews no one within the Police sample indicated that they felt that they needed to fill out the EDP form (Police complete EDP form). Post-change, respondents in the Police sample felt that such a change had occurred (strong unique Police).

- Police officers did not indicate that they wanted to increase communication within their organization before the introduction of the planned TS change (Police increased communication between frontline officers and supervisors). At Time 2, respondents in the Police sample felt that such a change had occurred (strong unique Police).

It is important to note that, while the Police did not express any desire to change their behavior within the TS before the planned changes were implement, many officers felt that they had changed their behavior in the TS in response to the planned TS change. More specifically, the Police reported that, post change, they filled out their EDP forms and that they communicated more often with their police counterparts who were involved in the PMI process. The fact that this view was not shared by the other stakeholders is important, particularly given the data showing that both of these other stakeholders wanted the Police to change. While the TS partners might not observe any communication changes that occurred within the police services, it is noteworthy that they did not observed changes with respect to EDP form completion rates.

Third, the results of our analysis supports the idea that the Social Service Agency observed a change that they did not mention they wanted within their own organization. More specifically:

- Although no Social Service Agency respondent indicated that they desired change in their behavior pre-change (Desired/Perceived Social Service Agency Change), they did indicate that they observed one organizational change.

- Community support workers did not mention they wanted to introduce an InSted program into the TS, even though many of these respondents felt that such a change had occurred (strong 
unique Social Service Agency) (Social Service Agency created InSted program to support $P M I)$.

Finally, in the Time 1 interviews no one in the Social Service Agency sample expressed any desire to change their behavior within the TS. Post-change, the Social Service Agency group felt that despite this, they had changed their behavior in the TS in response to the planned TS change. More specifically, they felt that the Insted program reflected a change in what they did within the TS.

We summarize these findings to the following five points:

- The Hospital and Police were classified as having Got Desired Change with respect to a new process that expedites service for PMIs within the Hospital.

- The Hospital and Social Service Agency were classified as having 'Thwarted Desires' when it comes to perceiving desired changes within the Police. More specifically, they did not observe Police behaviors changing as a result of mental health training or mandatory completion of EDP forms, even though they desired these changes.

- The Hospital was classified as having perceived an Unanticipated Change concerning more effective communication between TS stakeholders.

- The Police were classified as having perceived an Unanticipated Change associated with the completion of EDP forms.

- The Social Service Agency was classified as having perceived had an Unanticipated Change related to the implementation of the InSted program.

\subsection{WHAT CHANGED: A SENSEMAKING ANALYSIS OF THE PLANNED TS} CHANGE

The previous section used the Time 1 and Time 2 cross-sectional data to classify our TS stakeholders into three groups based on what they stated they wanted changed within the TS during 
the Time 1 interviews and what they observed had changed in the TS six month after the implementation of the of the planned TS change: Got Desired Change, Thwarted Desires, and Unanticipated Changes. To better understand how the introduction of the planned TS changes impacted the TS, we now turn to the panel data (i.e. data from 20 healthcare and 20 police respondents who participated in both rounds of interviews) and sensemaking theory to further explore how the individuals in our sample made sense of the planned TS change.

This section is divided into five subsections. The first subsection (Section 5.4.1) reviews relevant sensemaking literature and establishes why this approach is useful to this thesis. Second, Section 5.4.2 theoretically develops a framework for examining how individuals make sense of planned TS change. In Section 5.4.3, the methodology used to empirically test the framework is discussed. Section 5.4.4 presents the within-case analysis, and Section 5.4.5 presents the crosscase analysis of how individuals make sense of planned TS change.

\subsubsection{SENSEMAKING ABOUT PLANNED TS CHANGE}

Sensemaking is a process by which individuals can interpret past events (Weick, 1995) through "the ongoing retrospective development of plausible images that rationalize what people are doing" (Weick et al., 2005, p.409). Sensemaking activities involve individuals or groups (Sonenshein, 2010) challenging and reorienting their existing patterns of beliefs and assumptions to create new, more coherent understandings of their environment (Fiss \& Zajac, 2006). Research has shown that sensemaking can play a central role in changing behavior (Gioia \& Thomas, 1996), shift individuals' schemata during organizational transformation (Balogun \& Johnson, 2004), and lead to a change in interpretive organizational schemes (Balogun \& Johnson, 2005).

As the uncertainty invoked by change provides a salient context for sensemaking (Weick, 1995), scholars have taken to studying sensemaking within an organizational change context 
(Maitlis \& Sonenshein, 2010). In the rapidly growing body of literature on sensemaking about change, scholars have investigated sensemaking by examining how individuals respond to a change (e.g., Balogun et al., 2015; Kyriakidou, 2011; Riesenmy, 2010; Smollan, 2014; Stensaker \& Meyer, 2012; Stensaker et al., 2008), how they shape others' sensemaking about a change (e.g., Hensmans, 2015; Kezar, 2013; Marmenout, 2010), and how their discourse is affected by a change (e.g., Abolafia, 2010; Dawson, 2014; Islam, 2013; Reissner, 2011; Sonenshein, 2010; Teulier \& Rouleau, 2013).

Despite the plethora of research devoted to sensemaking about organizational change, we were unable to identify theoretical or empirical research concerning sensemaking about TS change. The lack of research devoted to sensemaking about TS change is unfortunate, as it provides little guidance to managers and employees in TSs (such as the case study TS) that are undergoing change.

While we were unable to find any research that spoke to the idea of sensemaking about TS change, our literature review did identify several studies that support the idea that individuals in different stakeholder groups within an organization (e.g., frontline, middle-managers, leaders) may make sense of change differently (Balogun, 2003; 2006; Dutton et al., 2001; Hope, 2010; Rouleau \& Balogun, 2011). As this finding implies that people from different stakeholders make sense of a change differently, we infer that individuals belonging to different TS stakeholders may make sense of a planned TS change differently.

\subsubsection{THEORETICAL DEVELOPMENT OF A TYPOLOGY: MAKING SENSE OF PLANNED TS CHANGE}

There is a large body of research that explores sensemaking. As such, a comprehensive review of this literature is beyond the scope of this thesis, which focuses on planned TS change. 
Accordingly, in this thesis, we restrict our discussion of the sensemaking literature to those authors who were formative in the theoretical development of the "making sense of planned TS change framework tested in this thesis: Balogun and Johnson (2004; 2005), Weick (1995), and Weick et al., (2005). Their relevant research is summarized below.

Balogun and Johnson (2005) identify two important concepts that they propose influence sensemaking: (1) old schemata, which refers to individuals' existing ways of thinking (i.e. the mental models individuals hold about the organization and the way individuals work together), and (2) sensemaking triggers, which refer to actions taken to deliver the planned changes (i.e., change goals, change interventions) and aspects of the designed change process perceived by the recipients to hinder rather than help the change process (i.e. 'flaws'). This theorization of sensemaking is similar to that of Lewin's theorization of drivers and barriers of change. In a way, sensemaking triggers drive individuals to develop new mental models, while old schemata can act as a barrier to the development of new mental models.

More specifically, when sensemaking triggers drive individuals to perceive cues in the organization that are not consistent with their old schemata, they engage in social processes of interaction (e.g., rumors, discussions) that result in the development of new schemata (Balogun \& Johnson, 2005). The idea of sensemaking triggers has relevance to this theory in those cases where our respondents perceive that the TS has changed, as we can assume that these individuals have recognized cues in their environment that were not consistent with their existing schemata (i.e. sensemaking triggers are in play) and developed new schemata to make sense of their situation. Other respondents in our sample did not, however, perceive that the TS has changed. According to Balogun and Johnson (2005), these individuals did not recognize any cues within the 
TS that were inconsistent with what they believed (i.e. their schema). This would suggest that individuals who did not perceive that the TS has changed maintains their old schemata.

Weick (1995) describes seven properties of sensemaking in his seminal book entitled Sensemaking in Organizations. These seven properties are summarized in Table 25.

\begin{tabular}{|l|l|}
\hline Sensemaking Property & Potential Implications of this Property on Thesis \\
\hline $\begin{array}{l}\text { Grounded in identity } \\
\text { construction }\end{array}$ & Sensemaking is influenced by individuals' identities \\
\hline Retrospective & Sensemaking is a hindsight analysis \\
\hline Enactive & Sensemaking translates meaning into action \\
\hline Social & Sensemaking involves consultation between individuals \\
\hline Ongoing and iterative & Sensemaking evolves \\
\hline $\begin{array}{l}\text { Driven by plausibility } \\
\text { rather than accuracy }\end{array}$ & $\begin{array}{l}\text { Individuals have preconceived notions of what should be } \\
\text { happening }\end{array}$ \\
\hline Focused on extracted clues & $\begin{array}{l}\text { Individuals observe environmental cues that further support } \\
\text { their beliefs or cause them to abandon certain beliefs }\end{array}$ \\
\hline
\end{tabular}

Table 25: Weick's (1995) Seven Properties of Sensemaking

Of the sensemaking properties noted above, the final two properties are of particular importance for the development of our typology of making sense of planned TS change.

The idea that sensemaking is driven by plausibility rather than accuracy suggests that individuals have preconceived notions of how something (i.e. what they are making sense about) is supposed to occur, and that these preconceived notions impact how they make sense of the situation. Applying this logic to the context under study in this thesis (i.e. a planned TS change) we posit that individuals' impressions of what they want to change in the TS (i.e. their preconceived notions of what might be happening) are likely to impact how they make sense of their situation.

According to Weick (1995), sensemaking is also based on the idea that individuals extract clues from the environment that may either support their existing system of beliefs or cause them to abandon certain beliefs. Applying this logic to sensemaking of a planned TS change, we could 
argue our stakeholders will either extract cues from the environment that confirm their beliefs with respect to the change and their stakeholder partners (i.e. see what they want to see, ignore the unanticipated) and/or make observations that cause them to change their beliefs either about the change or their partners.

These two properties of sensemaking identified by Weick lead us to theorize that four different types of sensemaking may occur within a TS undergoing a planned change as shown in Table 26. Membership in each of the four cells in our typology is based on: (1) whether or not the individual stated before any changes were implemented that they wanted to see a particular change introduced into the TS ("desired change at Time 1"), and (2) whether or not an individual mentioned that they had observed that the system had changed in a certain way six months after the implementation of the planned TS change ("perceived change at Time 2"). In the section below, we use theory developed by Balogun and Johnson (2005) and Weick (1995) to posit how these two sets of perceptions influence how respondents made sense of planned TS change.

\begin{tabular}{|c|c|c|c|}
\hline & & \multicolumn{2}{|c|}{ Perceived Change at Time 2} \\
\hline & & No & Yes \\
\hline \multirow{2}{*}{$\begin{array}{c}\text { Desired } \\
\text { Change at } \\
\text { Time } 1\end{array}$} & Yes & Thwarted Desires & Got Desired Change \\
\hline & No & Immaterial & Unanticipated Change \\
\hline
\end{tabular}

Table 26: Typology of Making Sense of Planned TS change

Thwarted Desires: Individuals were classified within this cell if they wanted a change to be implemented within the TS (i.e. desired change at Time 1) but did not perceive that such a change had been implemented (i.e. no perception of change at Time 2). We use the term 'Thwarted Desires' to describe how the people in this cell of our typology might view the changes they encounter. How would people who are in this situation make sense of change? Research by 
Balogun and Johnson (2005) would suggest that employees in this cell may not recognize cues that the organization (or TS) is changing because their old schemata is so strong that it prevents them from identifying environmental changes that are not consistent with their beliefs. Balogun and Johnson (2005) found, for example, middle managers who had old schemata that were supported with contracts and contract management procedures limited their discussions with others and prolonged their 'business as usual' perspective. As well, Balogun (2006) has suggested that employees may not recognize cues (of changes) in their environment when sensemaking triggers are ineffective (e.g. the implemented changes were ineffective due to organizational flaws).

Got Desired Change: Individuals were classified within this cell if they wanted a change to be implemented with the TS (i.e. desired change at Time 1) and then perceived that this had been implemented (i.e. perception of change at Time 2). We use the term 'Got Desired Change' to describe the change situation of the individuals in this cell. Balogun and Johnson's (2005) theorization of sensemaking would suggest that respondents would get their desired change if: (1) their goals were aligned with decision-maker goals and (2) sensemaking triggers were effective at challenging individuals' old schemata. Balogun (2006) argues that this is most likely to occur when decision-makers use lateral and informal communication between peers and align interpretations of the change through sharing and developing interpretations through many different genres.

Unanticipated Change: Individuals were classified within this cell if they perceived that a change had been implemented (i.e. perception of change at Time 2) but did not mention they wanted this change to be implemented (i.e. no desire for change at Time 1). We use the term 'Unanticipated Change' to describe how people in this cell might view the change. How would individuals who encounter a change that they perceive as unanticipated make sense of change? 
Balogun and Johnson (2005) theorize that old schemata inform and sensemaking triggers lead to emergent change outcomes, which refer to "the realization of a new pattern of organizing in the absence of explicit, a priori intentions" (Orlikowski, 1996, p.65). Given that emergent changes may result in welcomed as well as unwelcomed outcomes (Armenakis \& Bedeian, 1999), individuals in this quadrant may make sense of unanticipated changes by thinking that they result in positive or negative outcomes - or what Balogun and Johnson (2005) refer to as 'congruent' and 'counteracting' change consequences.

Immaterial: Individuals were classified within this cell if they did not mention they wanted a change to be implemented with the TS (i.e. no desire for change at Time 1) and did not observe any change in this area after changes had been implemented (i.e. no perception of change at Time

2). We use the term 'Immaterial' to describe how the people in this cell of our typology would view the planned change. Given that the individuals in this cell have shown little awareness of the change that may be happening around them (i.e. did not mention they wanted a change, did not mention that they observed the change), they are not likely to be making sense of the change. According to Weick (1995), these individuals may not be enacting, socializing, and observing changes in their environment, which would prevent them from developing schemata.

\subsubsection{METHODOLOGY: MAKING SENSE OF PLANNED TS CHANGE}

We followed Eisenhardt's (1989) case study theory building approach to test our typology describing how individuals make sense of planned TS change. For the purposes of this analysis, we define a case as the perceptions associated with a desired or perceived change to the TS (see Table 24 for list of possible cases). Following Eisenhart's (1989) recommendations for theoretical, not random, selection of cases, we selected four cases for inclusion in this stage of our analysis. The cases were selected to ensure that we included a case that addressed an interorganizational 
change, a Hospital change, and a Police change. This selection criterion resulted in the decision to test our typology using panel data relating to the following four changes: (1) increased interorganizational communication (i.e. an interorganizational change), (2) a new ED process that expedites PMIs brought in by Police (i.e. a Hospital change), (3) increased engagement of MHESU (i.e. a Hospital change), and (4) mandatory completion of EDP forms (i.e. a Police change).

For each case, we classified respondents into one of the four cells in our typology based on how they answered the following two interview questions: 'what needs to change in the PMI process?' (Time 1) and 'what have you observed has changed in the PMI process?' (Time 2). For example, if the respondent mentioned s/he wanted increased interorganization communication but did not perceive that it had occurred, we would classify that respondent in the Thwarted Desires cell. We then used incident-to-incident coding (Charmaz, 2006) to analyze the comments provided by individuals in each of the three cells of our typology in response to the question asked in the Time 2 interviews with respect to what changes they had observed. We created and assigned firstorder codes to summarize themes from informants' responses. All codes were emergent. We used constant comparison to ensure that new codes were distinct from other codes and identify any potential links between codes (Charmaz, 2006).

It should be noted that respondents in the immaterial cell are excluded from any of the follow up analysis presented in this section of the thesis. Practically speaking, as this cell represents individuals who did not mention that they wanted a change at Time 1 nor perceived certain changes had occurred at Time 2, we were unable to find any comments from these individuals that helped us understand their sensemaking. We also note that individual responses to the Time 1 interview on desired changes within the TS were only used to classify respondents in one of the four cells (i.e. they were not part of the analysis reported in this section). 
In the following section (Section 5.4.4), we perform a within-case analysis and identify first-order codes associated with each of the four cases. We then group similar first-order codes to form second-order categories. We provide exemplary quotes to support themes that have emerged within three of the quadrants (excluding immaterial) based on the comments of respondents belonging to each quadrant. We consider a theme emerging from the data when the theme is supported by the comments provided by at least three respondents. Section 5.4.5 presents a cross-case analysis.

\subsubsection{EMPIRICAL ASSESSMENT OF MAKING SENSE OF PLANNED TS CHANGE: WITHIN-CASE ANALYSIS}

This subsection conducts a series of within-case analyses. More specifically, we empirically assess the theoretical framework of making sense of planned TS change by using data associated with four changes: (1) increased interorganizational communication (Section 5.4.4.1), (2) an ED process that expedites PMIs brought in by Police (Section 5.4.4.2), (3) improved MHESU engagement (Section 5.4.4.3), and (4) mandatory completion of EDP form by Police (Section 5.4.4.4).

\subsubsection{CASE ONE: ANALYSIS OF PERCEPTIONS OF INCREASED INTERORGANIZATIONAL COMMUNICATION}

The first case involves an emergent interorganizational change. Evidence from the TS case study supports the idea that the planned TS change has increased communication between various partners within the TS. In particular, sixty-two percent of the Time 2 sample commented that they thought communication had increased between TS stakeholders post-change. Interestingly, this particular change may be considered emergent, as none of the four specific changes included in the planned TS change had this objective. Nevertheless, the evidence from this thesis suggests that 
this change was perceived by many of the respondents in our study. Accordingly, we use data associated with this change as the first case to test our sensemaking framework.

Respondents were classified into the four cells of our typology based on the examination of the responses according to two criteria. The first criterion establishes whether or not (i.e. yes or no) the respondent mentioned they wanted increased interorganizational communication at Time 1 (before the introduction of the planned TS change), whereas the second criterion shows whether or not (i.e. yes or no) the respondent said that they perceived that interorganizational communication had increased over time (i.e. at Time 2). Based on these two criteria, respondents were classified as Got Desired Change, Thwarted Desires, Unanticipated Change, or Immaterial as shown in in Table 27. 


\begin{tabular}{|c|c|c|c|}
\hline \multicolumn{2}{|c|}{} & \multicolumn{2}{c|}{ Perceived Improved Interorganizational Communication } \\
(Time 2)
\end{tabular}

Table 27: Making Sense of Improved Interorganizational Communication

The distribution of the interviewees into the four cells is noteworthy. In this case, twentytwo individuals (half the sample) were categorized in the Unanticipated Change cell, thirteen (35\% of Hospital sample, $30 \%$ of Police sample) in the Immaterial cell, and five (5\% of Hospital sample, $20 \%$ of Police sample) in the Got Desired Change cell. No one was categorized as belonging to the Thwarted Desires cell with respect to perceptions of increased communication between TS stakeholders. It is also important to note that the proportions of health care and police respondents in each of these four cells were approximately equal, which suggests that they made sense of this change in the same manner. In the following subsections, we present data of the responses given by the respondents that we categorized in the following two cells in our typology: Got Desired Change and Unanticipated Change.

Got Desired Change. Data analysis identified five respondents as meeting our criteria for inclusion in the Got Desired Change cell of our making sense of planned TS change typology (see Table 28). Analysis of the comments provided at Time 2 by the individuals in this group identified one first-order code. More specifically, our analysis determined that the four police officers and the one healthcare worker in the Got Desired Change group made sense of the changes introduced into the TS by thinking that the increased interorganizational communication resulted in more 
effective communication between TS partners. The following quotes exemplify this type of comment that we labeled: 'more effective communication between police officers and ED staff'.

“There's more open communication when they're in the department and on the floor and when the sergeants come and, or give us a call..." (Hospital Resource Nurse Time 2)

"Some of the changes have... created more effective communication I guess as a team."

(Police Sergeant Time 2)

\begin{tabular}{|c|c|c|c|c|c|c|}
\hline \multirow{2}{*}{$\begin{array}{c}\text { Got Desired Change: Increased } \\
\text { Interorganizational Communication }\end{array}$} & \multicolumn{2}{|c|}{$\begin{array}{c}\text { Total } \\
(n=40)\end{array}$} & \multicolumn{2}{|c|}{$\begin{array}{c}\text { Hospital } \\
(\mathbf{n}=\mathbf{2 0})\end{array}$} & \multicolumn{2}{|c|}{$\begin{array}{l}\text { Police } \\
(\mathbf{n}=\mathbf{2 0})\end{array}$} \\
\hline & $\#$ & $\%$ & \# & $\%$ & $\#$ & $\%$ \\
\hline $\begin{array}{l}\text { More effective communication between police } \\
\text { officers and ED staff }\end{array}$ & 5 & 13 & 1 & 5 & 4 & 20 \\
\hline $\begin{array}{r}\text { More effective communication between police } \\
\text { officers and ED staff }\end{array}$ & 5 & 13 & 1 & 5 & 4 & 20 \\
\hline
\end{tabular}

Table 28: Got Desired Change: Increased Interorganizational Communication

Unanticipated Change. Analysis of the comments provided at Time 2 by the twentytwo respondents from the panel data that we classified into the Unanticipated Change cell of our making sense of planned TS change typology are shown in Table 29 and discussed below. Based on the comments provided at Time 2 from these twenty-two respondents, we identified three different ways (i.e. first-order codes) that they made sense of the planned TS change by examining their perceptions of increased communication between TS stakeholders. Of these three ways that respondents made sense of the planned TS change, one was linked with improved service to PMIs, while the other two were related with increased Police demands on Hospital. 


\begin{tabular}{|c|c|c|c|c|c|c|}
\hline \multirow{2}{*}{$\begin{array}{c}\text { Unanticipated Change: Increased } \\
\text { Interorganizational Communication }\end{array}$} & \multicolumn{2}{|c|}{$\begin{array}{c}\text { Total } \\
(n=40)\end{array}$} & \multicolumn{2}{c|}{$\begin{array}{c}\text { Hospital } \\
\text { (n=20) }\end{array}$} & \multicolumn{2}{c|}{$\begin{array}{c}\text { Police } \\
(n=20)\end{array}$} \\
\cline { 2 - 8 } & $\#$ & $\%$ & $\#$ & $\%$ & $\#$ & $\%$ \\
\hline Improved Service to PMIs & $\mathbf{1 0}$ & $\mathbf{2 5}$ & $\mathbf{2}$ & $\mathbf{1 0}$ & $\mathbf{8}$ & $\mathbf{4 0}$ \\
\hline Improved service to PMIs & 10 & 25 & 2 & 10 & 8 & 40 \\
\hline Increased Police Demands on Hospital & $\mathbf{1 0}$ & $\mathbf{2 5}$ & $\mathbf{1 0}$ & $\mathbf{5 0}$ & $\mathbf{0}$ & $\mathbf{0}$ \\
\hline Police pressure ED staff to expedite PMIs & 7 & 18 & 7 & 35 & 0 & 0 \\
\hline Police distract ED staff & 3 & 8 & 3 & 15 & 0 & 0 \\
\hline
\end{tabular}

Table 29: Unanticipated Change: Increased Interorganizational Communication

Our analysis suggests that half of the respondents in the Unanticipated Change cell made sense of the planned TS change by interpreting it as an improved service to PMIs. More specifically, two healthcare workers and eight police officers, when discussing their observations of the change, spoke about how communicating with their TS partner had better prepared them for dealing with PMIs in the future. We gave the first-order code, 'Improved service to PMIs', to comments that were similar to the following:

"I think we are talking through each other's issues a lot better... and I think this has allowed the project to go beyond just moving through the system [PMI process]." (Hospital Manager)

“I think everybody's keeping you in the loop more often... and we're more prepared to deal with these situations." (Police Constable)

Healthcare respondents within the Unanticipated Change group made two type of comments suggesting that police officers were placing more demands on healthcare workers: (1) Police pressured ED staff to expedite PMIs, and (2) Police distracted ED staff. Seven healthcare workers made comments, such as those below, which were related to police officers pressuring ED staff to expedite PMIs. This type of comment was given the following label: 'Police pressure ED staff to expedite PMIs'. 
"They [police officers] know how to escalate things... when we're approaching the two-hour mark they come, they talk to me, and they say 'we're approaching the two-hour mark, how's it looking?' And usually I say not good (laughter)... Then the sergeants call me, or they'll come and see me, and they'll ask 'what's going on'. So it's been escalated to the sergeants..." (Hospital ED Nurse Time 2)

"I think that they [police officers] feel now that they can put pressure on the ED when waiting for a time period and they feel it gives them the backup to be able to put pressure on the nurse or call their sergeant to escalate it. If their patients are not being seen in that timely fashion.” (Hospital ED Physician Time 2)

Our analysis suggests that, in the Unanticipated Change group, a few of the healthcare workers ( $\mathrm{n}=3$ ) made sense of the planned TS change by interpreting increased interorganizational communication as a result of police officers distracting ED staff when they were assessing or treating patients who were not PMIs. This finding points to the idea that police officers who are trying to expedite PMIs within the Hospital can be distracting to ED staff, and make it more difficult for healthcare workers to perform their other duties. An example of this type of comment, which was labeled 'Police distract ED staff', was given by an ED physician as follows:

"It can be distracting for the charge nurse and the physician when they are managing some really sick patients and the mental health patient issue becomes added to that because it's hitting the two-hour mark. The police are now up in arms, the police are calling the sergeant, and the sergeant is now calling the charge nurse. The charge nurse may be dealing with something that may be more pressing at the time but now has to refocus her attention or deal with the police as well."

The comments above reflect the notion that, while healthcare workers may have changed their 
behavior as per the intervention and escalation policy, they make sense of their new situation as one in which the Police are pressuring and distracting them from their work. In other words, they view the increase in communications in a negative light, whereas the police view their actions more positively (i.e. contributing to better service to PMI).

We summarize our analysis of how respondents made sense of increased interorganizational communication as follows:

- Respondents from both TS stakeholder groups made sense of getting this desired change by focusing on how the planned TS change had increased the effectiveness of TS communication processes (i.e. made sense of the change in a positive way).

- Police officers made sense of this Unanticipated Change by in a way that led them to feel that the changes in communication within the TS had benefited others (the PMIs) but not themselves.

- Healthcare workers made sense of this Unanticipated Change in a way that led them to feel that their resources were strained and that Police were placing more demands on them (i.e. made sense of the change in a negative way).

\subsubsection{CASE TWO: ANALYSIS OF PERCEPTIONS OF AN ED PROCESS THAT EXPEDITES SERVICE FOR PMIS}

The second case involves a planned Hospital change. One of the four specific changes agreed on by decision-makers involved the implementation of the Intervention and Escalation Policy (see Section 5.1.2.4). This planned change was implemented by healthcare workers and designed to decrease the length of time PMIs have to wait in the Hospital to be assessed by a doctor. Quantitative evidence (see Section 5.2) supports the idea that there has been a decrease in the length of time PMIs have to wait to be assessed by a physician in the Hospital. Analysis of the 
qualitative data (see Section 5.3.1) indicates that respondents have perceived PMIs (and police officers) waiting for shorter periods of time in the Hospital after the planned TS change was implemented. This section uses panel data regarding perceptions of an ED process that expedites service for PMIs to get a better understanding how respondents made sense of the planned TS change.

Respondents were classified into the four cells of our making sense of change typology based on their responses to two questions: (1) whether or not they mentioned that they wanted shortened waiting time for PMIs and Police at Time 1, and (2) whether or not they said that they perceived a new process that expedites service for PMIs in the ED post-change. The results of this classification process are shown in Table 30 and discussed below.

\begin{tabular}{|c|c|c|c|}
\hline \multicolumn{2}{|c|}{} & \multicolumn{2}{c|}{ Perceived a New Process that Expedites Service for PMIs in the ED } \\
(Time 2)
\end{tabular}

Table 30: Making Sense of Perceptions of an ED Process that Expedites Service for PMIs

The majority of respondents (40\% of Hospital sample, $75 \%$ of Police sample) were categorized in the Got Desired Change cell. Eight respondents were classified as belonging in the Thwarted Desires cell (15\% of Hospital sample, 25\% of Police sample), and the Unanticipated Change cell (40\% of Hospital sample, $0 \%$ of Police sample). When we compare the number of Police and Hospital respondents within each of these cells, we see that, while the respondents from the Police sample outnumbered their counterparts from the Hospital sample in the Got Desired Change cell, the reverse was true for the Unanticipated Change cell (i.e. more Hospital than 
Police). In the following subsections, we analyze the responses given by respondents classified in each of the following cells: Got Desired Change, Thwarted Desires, and Unanticipated Change.

Got Desired Change. We present and discuss the analysis of the comments provided at Time 2 by the twenty-four respondents from the panel data that we classified into the Got Desired Change cell (see Table 31). Based on the comments provided at Time 2 from these twenty-four respondents, we identified six different ways (i.e. first-order codes) that they made sense of the planned TS change. Three of these six first-order codes were linked with improvements for the Police, two were associated with improvements for the Hospital, and related to how respondents regarded each other's responsibilities within the PMI process.

\begin{tabular}{|c|c|c|c|c|c|c|}
\hline \multirow{2}{*}{$\begin{array}{c}\text { Got Desired Change: An ED Process } \\
\text { that Expedites Service for PMIs }\end{array}$} & \multicolumn{2}{|c|}{$\begin{array}{l}\text { Total } \\
(n=40)\end{array}$} & \multicolumn{2}{|c|}{$\begin{array}{l}\text { Hospital } \\
(\mathbf{n}=\mathbf{2 0})\end{array}$} & \multicolumn{2}{|c|}{$\begin{array}{l}\text { Police } \\
(n=20)\end{array}$} \\
\hline & \# & $\%$ & $\#$ & $\%$ & $\#$ & $\%$ \\
\hline Improved Police Operations & 22 & 55 & 7 & 35 & 15 & 75 \\
\hline $\begin{array}{r}\text { Police return to service in community in a timely } \\
\text { manner }\end{array}$ & 22 & 55 & 7 & 35 & 15 & 75 \\
\hline $\begin{array}{r}\text { Police have greater understanding of their role in } \\
\text { PMI process }\end{array}$ & 6 & 15 & 1 & 5 & 5 & 25 \\
\hline Resource nurse supports Police in ED & 3 & 8 & 0 & 0 & 3 & 15 \\
\hline Improved Hospital Operations & 6 & 15 & 6 & 30 & $\mathbf{0}$ & $\mathbf{0}$ \\
\hline Greater Hospital awareness of PMIs in the ED & 4 & 10 & 4 & 20 & 0 & 0 \\
\hline $\begin{array}{r}\text { More timely communication between ED staff and } \\
\text { psychiatrists }\end{array}$ & 4 & 10 & 4 & 20 & 0 & 0 \\
\hline $\begin{array}{l}\text { Improved understanding of each other's } \\
\text { responsibilities in PMI process }\end{array}$ & 4 & 10 & 1 & 5 & 3 & 15 \\
\hline $\begin{array}{r}\text { Improved understanding of each other's } \\
\text { responsibilities in PMI process }\end{array}$ & 4 & 10 & 1 & 5 & 3 & 15 \\
\hline
\end{tabular}

Table 31: Got Desired Change: An ED Process that Expedites Service for PMIs

Analysis of the qualitative data suggests that respondents in the Got Desired Change cell (most of whom were police officers) made sense of the change in three different ways: (1) it allowed police to return to service in the community promptly, (2) it increased their understanding of their role in the PMI process, and (3) it gave them access to a resource nurse at the Hospital to 
support their work. First, our analysis suggests that virtually all of the police officers in the panel data set $(75 \%)$ and one in three of the healthcare workers $(35 \%)$ made sense of the planned TS change by thinking that the new behavior associated with the intervention and escalation policy benefited the Police. In particular, these respondents noted that the policy made it possible for police officers to leave the Hospital in a timelier manner post-change than pre-change. This type of comment was given the first-order code: 'Police return to service in the community in a timely manner'. Example comments given this label are provided below.

"This process seems to work a lot better than their older one, a little faster too. Wait times have decreased so for the most part we can return to our post quicker." (Police Constable Time 2)

"We certainly have a better method for assessing these patients to get the police out of here quicker.” (Hospital Resource Nurse Time 2)

When talking about their observations of an ED process that expedites service for PMIs, respondents in the Got Desired Change cell of the typology ( $5 \%$ of Hospital sample, $25 \%$ of Police sample) made comments that suggested they made sense of the change as one that provided police officers with a better understanding of their role in the PMI process. This type of comment was given the label: 'Police have a greater understanding of their role in PMI process'. One sergeant spoke broadly about this improved understanding by stating that:

"I think some of the changes have just allowed us a better understanding of our job functions ... and how our job relates to $[P M I s]$... allowed us to place expectations when at the Hospital."

One resource nurse provides a little more explanation regarding how that improved Police understanding may impact healthcare workers by describing her observations post-change: 
“There's a little bit more understanding from what we're dealing with... they'll call and say, somebody's been there for three and a half hours waiting to be assessed but now they'll come in, they'll look around, they acknowledge, they give us a little acknowledgement by yes, wow, it's very busy, if there's anything you can do to help us out, it's appreciated and I think it's a more, it feels more friendly opposed to maybe early on in the process it was more, when the sergeant would call it felt more like they're interrogating you and you yourself is going to be under arrest to now, they come in and it's more of a friendly, different approach with a better personal communication."

The above comments reflect police officers making sense of the changes associated with expediting the Police wait times in the ED by feeling that it has allowed police officers to have a greater understanding of their environment, and how their behaviors are interconnected with others within the TS (i.e. interpreted a change that benefited them in a way that meant it benefited the system).

Respondents that met our criteria for Got Desired Change also made comments that suggested they made sense of the planned TS change as one where healthcare workers provided additional support for police officers within the ED and gave priority to PMIs who were brought in by the police. More specifically, three Police respondents ( $0 \%$ of Hospital sample, $15 \%$ of Police sample) commented that the resource nurse acted as a liaison within the ED for the Police. These observations were labeled: 'resource nurse supports Police in ED'. For example, one sergeant discussed his post-change observations by stating:

"Now there's a resource nurse available for us to liaise with to ensure that if our officers are beyond the two-hour time limit that they I guess for lack of a better term be pushed up 
as far as priority is concerned so that has certainly helped things a lot. So we know what's happening..."

It is interesting to note that Police interpret the availability of this additional resource (i.e. the nurse) as a cue that the Hospital is responding to the needs of the police, even though this change is also likely available to benefit the PMI by accelerating their progress through the system.

When discussing their observations of an ED process that expedites service for PMIs, six respondents made comments suggesting that the Hospital benefited from the planned TS change. Interestingly, all six of these individuals were healthcare workers. These healthcare respondents spoke about two ways that the Hospital's operations may have benefited from this aspect of the planned TS change: (1) greater awareness of PMIs in the ED, and (2) more timely communication between ED and psychiatrists. Details on each of these two first-order codes are provided below.

When we asked respondents what they observed has changed over time, four healthcare respondents within the Got Desired Change group made comments that suggested healthcare workers were more aware of their environment within the ED, and this greater awareness has improved their operations. It would seem that, based on the respondents' comments, these respondents made sense of the planned TS change by interpreting shorter wait times for PMIs as a result of healthcare workers having a greater awareness of PMIs within the ED. For comments of this nature, we provided the first-order code: 'greater Hospital awareness of PMIs in the ED'. For example, one manager within the Hospital described her observations of the change in the following way:

"I think we've got a better way of identifying that this patient population is in the ED and escalating a process when we're reaching that target point of two hours. I think that just ensuring that people are aware because I think in the past basically...you know they're there 
but without anybody saying, okay, it's been this long, I think you feel left without anybody really following up. So I think that's been a very good process that's been implemented that helps to identify them."

Our analysis also shows that respondents we classified as Got Desired Change made sense of the planned TS change by thinking that healthcare workers were able to expedite PMIs by improving communication within the Hospital. More specifically, four healthcare respondents discussing their observations of the change at Time 2 spoke about a timelier communication between nurses and doctors within the ED and psychiatrists. These comments were coded as 'more timely communication between ED staff and psychiatrists'. For instance, a resource nurse described his observations of the intervention and escalation policy stating:

"I think it's forced the emergency to have better communication with psychiatrist on a more timely basis...To what's happening up to the minute in the ED in regards to psychiatric patients to be assessed by psychiatry, as opposed to, I would say a year ago they were waiting probably 24 hours in the ED for a psychiatric assessment and I would say, now, it's improved quite a bit, I would say sometimes it's relatively soon after or up to eight to 12 hours after, so, probably improved $50 \%$, this last year."

Finally, our analysis of the Time 2 data suggests that 4 respondents in the Got Desired Change cell thought that the planned TS change resulted in the Hospital and the Police having a better understanding of each other's responsibilities within the PMI process. It would seem that the respondents made sense of the new behaviors resulting from the planned TS change as contributing to their understanding of their partner's role in the PMI process. This type of comment was labeled: 'improved understanding of each other's responsibilities in PMI process'. A sergeant made sense of the implementation of the intervention and escalation policy by saying: 
"I think that to a certain degree that the police and hospital staff are a little bit more on the same page as far as what our responsibilities are and as far as streamlining kind of more of an effective or quicker process as far as getting our officers back on the road."

Thwarted Desires. Data analysis identified eight respondents as meeting our criteria for inclusion in the Thwarted Desires cell of our making sense of the planned TS change typology (see Table 32). Examination of the post-change comments provided by these three healthcare and five Police respondents reveals three different ways (i.e. first-order codes) that they made sense of the planned TS change based on their perceptions of an ED process that expedites service for PMIs. All three of these first-order codes relate to ways in which the ED restrains changing the PMI process.

\begin{tabular}{|c|c|c|c|c|c|c|}
\hline \multirow{2}{*}{$\begin{array}{c}\text { Thwarted Desires: An ED Process that } \\
\text { Expedites Service for PMIs }\end{array}$} & \multicolumn{2}{|c|}{$\begin{array}{c}\text { Total } \\
\text { (n=40) }\end{array}$} & \multicolumn{2}{c|}{$\begin{array}{c}\text { Hospital } \\
\text { (n=20) }\end{array}$} & \multicolumn{2}{c|}{$\begin{array}{c}\text { Police } \\
\text { (n=20) }\end{array}$} \\
\cline { 2 - 8 } & $\#$ & $\%$ & $\#$ & $\%$ & $\#$ & $\%$ \\
\hline ED Barriers to Changing PMI Process & $\mathbf{8}$ & $\mathbf{2 0}$ & $\mathbf{3}$ & $\mathbf{1 5}$ & $\mathbf{5}$ & $\mathbf{2 5}$ \\
\hline ED doctors were not expediting PMIs & 5 & 13 & 1 & 5 & 4 & 20 \\
\hline Insufficient ED staff to expedite PMIs & 3 & 8 & 1 & 5 & 4 & 20 \\
\hline PMI priority remains low in ED & 3 & 8 & 1 & 5 & 4 & 20 \\
\hline
\end{tabular}

Table 32: Thwarted Desires: An ED Process that Expedites Service for PMIs

When asked what changes they perceived within the TS, respondents classified within the Thwarted Desires cell discussed three ways that the felt that the ED may be restraining changes to the PMI process: (1) ED doctors were not expediting PMIs, (2) there was insufficient ED staff to expedite PMIs, and (3) the priority of the PMI remained low in the ED. Details on each of these three first-order codes are provided below.

Our analysis suggests that one healthcare worker and four police officers classified in the Thwarted Desires cell made sense of the planned TS change by thinking that their desired change 
associated with a process that expedites PMIs was not achieved because doctors refused to expedite PMIs brought in by police officers. We provided the first-order code 'ED doctors were not expediting PMIs' to comments that reflecting the idea that it was the doctor's that were impeding the desired changes to expedite PMIs. For example, a manager within the MHESU discussed her observations by commenting:

"Initially there was some change noted but ultimately, over time, things have broken down. So initially, there was some identifiable change, patients were offloaded more efficiently so that the police could be on their way. It sounded all well and good however the problem is that there were not enough physicians in the sense. Doctors would say-well look it doesn't matter to me whether they are here by the police or not, they just have to wait their turn. And that consequently results in the huge backup because it is so busy there. So doctors stopped expediting them... and nothing changed."

Our analysis suggests that the five respondents in this cell (5\% of Hospital sample, $20 \%$ of Police sample) made sense of the planned TS change by attributing the issue of not observing their desired change (i.e. a process that expedites PMIs) to the fact that there was insufficient staff within the ED to expedite the PMIs. In other words, these respondents made sense of their situation by identifying insufficient ED staff as the barrier that was impeding the change. For example, a police constable provided the following comment that we labeled: 'insufficient ED staff to expedite PMIs'.

"We need more, maybe practical nurses that have the ability to sign a form, the practical nurses, they have the ability, they have signing powers just like doctors do, so I don't know if we need more of those, just something to expedite the process."

Other respondents in the Thwarted Desires cell (5\% Hospital sample, 20\% Police sample) 
made comments supporting the notion that the planned TS change did not result in decreased wait times because the priority of the PMI did not increase post-change. These respondents made sense of their situation by attributing a lack of desired change to the idea that the PMI's priority within the ED remained low. We provided this type of comment the label: 'PMI priority remains low in ED'. One police constable spoke about his observations within the PMI process post-change: "Our priority [in the ED] is still low in terms of comparing that to somebody in medical, and I understand, somebody who is in medical distress compared to what we have, we have somebody who is, but isn't in dire need of medical attention."

Unanticipated Change. Table 33 shows our analysis of eight respondents that we classified within the Unanticipated Change cell of our typology. Our review of the post-change comments made by these eight respondents, all of them in the Hospital sample, suggests that there were four different ways (i.e. first-order codes) that they made sense of the planned TS change based on how they interpreted changes associated with the ED process that expedites service for PMIs. Three of these first-order codes were linked with the strain put on their resources, whereas the fourth was related to Police putting demands on the Hospital. 


\begin{tabular}{|c|c|c|c|c|c|c|}
\hline \multirow{2}{*}{$\begin{array}{c}\text { Unanticipated Change: An ED Process } \\
\text { that Expedites Service for PMIs }\end{array}$} & \multicolumn{2}{|c|}{$\begin{array}{c}\text { Total } \\
(n=40)\end{array}$} & \multicolumn{2}{|c|}{$\begin{array}{c}\text { Hospital } \\
(\mathrm{n}=\mathbf{2 0})\end{array}$} & \multicolumn{2}{|c|}{$\begin{array}{l}\text { Police } \\
(n=20)\end{array}$} \\
\hline & $\#$ & $\%$ & \# & $\%$ & \# & $\%$ \\
\hline Hospital Resources Strained & 8 & 20 & 8 & 40 & $\mathbf{0}$ & $\mathbf{0}$ \\
\hline Insufficient bed availability for PMIs & 5 & 13 & 5 & 25 & 0 & 0 \\
\hline $\begin{array}{r}\text { Not enough MHESU staff to form PMIs } \\
\text { appropriately }\end{array}$ & 3 & 8 & 3 & 15 & 0 & 0 \\
\hline Security guarded resources strained & 3 & 8 & 3 & 15 & 0 & 0 \\
\hline Police made demands without providing support & 4 & 10 & 4 & 20 & $\mathbf{0}$ & $\mathbf{0}$ \\
\hline Police made demands without providing support & 4 & 10 & 4 & 20 & 0 & 0 \\
\hline
\end{tabular}

Table 33: Unanticipated Change: An ED Process that Expedites Service for PMIs

Respondents that were classified within the Unanticipated Change cell made sense of the planned TS change by thinking that the new efforts to reduce PMI and Police wait times were straining Hospital resources in the following three ways: (1) insufficient bed availability for PMIs, (2) not enough MHESU staff to form PMIs appropriately, and (3) security guard resources strained. Details on each of these three ways are provided below.

Five respondents in this group made sense of the planned TS change by linking the intervention and escalation policy to the idea that the Hospital's resources were strained regarding bed availability. These respondents all talked about how the bottleneck in the PMI process was related to bed space in the Hospital. Such comments were grouped and given the label: 'Insufficient bed availability for PMIs'. For example, one security guard described his sensemaking of the intervention and escalation policy by saying:

"There is a little bit of a bottlenecking with the push to get the police officers out. Just the fact that the hospital is quite inundated with not enough bed space for the mental health population that we treat here."

Three of the ED staff within this cell of the typology made comments suggesting that they felt that, in the rush to get Police out of the Hospital quicker, some of the PMIs were getting formed 
inappropriately. It appears that these respondents made sense of the change (or, in their view, lack thereof) as limitations associated with an understaffed MHESU. More specifically, they noted that, post-change, the staff was unable to adequately assess the PMI and report their findings to the doctor before the doctor decided to form or not form the PMI. This type of comment was given the following label: 'Not enough MHESU staff to form PMIs appropriately'. One resource nurse in the Hospital expressed this concern when he said:

"I feel bad for some of the patients because we still don't have enough crisis workers and mental health nurses for a better method on assessing these patients, so, I feel, unfortunately, some of these patients get formed just to get the police out of there quicker... and that form affects people for the rest of their lives."

All of the Hospital's security guards in our sample made sense of the planned TS change by thinking that police officers leaving the Hospital earlier meant that security guards would be working more. This type of comment, such as the comment made by the security guard below, was given the label: 'security guard resources strained'.

"So what happens is after the police officers leave, security takes over for them, but these patients sometimes are being released in areas that are not secure and require security. It's a large strain on our resources. So we've gone from having to have normally one or two security officers in the ED to having to have three at all times."

Lastly, four healthcare respondents within the Unanticipated Change group made comments that suggested they made sense of the planned TS change by thinking that Police were placing more demands on them without providing the necessary support to help them change. 'Police made demands without providing support' was the label given to this type of comment. For example, one resource nurse recounted a previous interorganizational change where 
paramedics provided financial support to the Hospital so that they could expedite patients from the paramedics. This resource nurse notes that in this TS change, the Police were not providing the same support.

"It's frustrating for me to know that the paramedics have paid for a nurse, a designated offload nurse, and we have some of the best times in our region for paramedics. However, we're expected to meet these targets for the Police and they haven't given us anything... I don't know if there's something that happened behind the scenes, but I don't see any support, in terms of financial or in terms of like, changing the process that would help us meet those targets better."

The following key observations can be made from our analysis of how the different TS stakeholders made sense of the new ED process that expedites service for PMIs:

- Respondents in the "Desired Change" cell of the typology engaged in sensemaking activities that resulted in them viewing the change as one that had positively impacted the operations of their organization (i.e., police officers felt that the change improved Police operations, healthcare workers perceived that this change improved Hospital operations) and had given them a better understanding of their own role and their TS partner's role in the PMI process.

- Police officers in the Thwarted Desires cell of the typology focused on cues that lead them to feel that they had not got the change they wanted because of barriers within the Hospital (i.e. their partner had prevented the implementation of a change they wanted).

- Healthcare workers in the Unanticipated Change cell of the typology made sense of the unexpected aspects of the planned TS change by focusing on how the change had negatively impacted their organization (i.e. their resources were strained and that Police 
were placing more demands on them without providing support).

\subsubsection{CASE THREE: ANALYSIS OF PERCEPTIONS OF IMPROVED MHESU ENGAGEMENT}

The third case involves an emergent Hospital change. Data summarized in Chapter 5 suggests that the planned TS change affected how the Mental Health Emergency Services Unit (MHESU; e.g., crisis workers, mental health nurses, psychiatrists) was involved in the PMI process. Although none of the four specific changes that were part of the planned TS change were designed to impact the MHESU, after the changes were implemented, some of the respondents noted that they had observed considerable changes to the MHESU. The fact that some respondents perceived MHESU staff more engaged in the PMI process after the planned TS change was implemented is most likely the result of applying the intervention and escalation policy within the ED, which facilitated collaboration between ED and MHESU staff (see Section 5.1.2.4 for more detail). This section examines the panel data linked with perceptions of improved MHESU engagement to gain a better understanding of how respondents made sense of the planned TS change.

Respondents were classified into the four cells of our typology by looking at their response to two questions: whether or not the respondent stated that they wanted increased MHESU engagement in the Time 1 interviews, and whether or not the respondent said that they perceived improved communication between ED and MHESU staff in the Time 2 interviews. Based on the responses that respondents gave to these two questions we classified respondents into the Got Desired Change, Thwarted Desires, Unanticipated Change, or Immaterial cells. As shown in Table 34. 


\begin{tabular}{|c|c|c|c|}
\hline & \multicolumn{2}{|c|}{$\begin{array}{l}\text { Perceived Improved Communication between ED and MHESU } \\
\text { (Time 2) }\end{array}$} \\
\hline & & No & Yes \\
\hline \multirow{2}{*}{$\begin{array}{l}\text { Desired } \\
\text { Increased } \\
\text { MHESU } \\
\text { Engagement } \\
\text { (Time 1) }\end{array}$} & Yes & $\begin{array}{l}\text { Thwarted Desires } \\
\text { Hospital: } 13(65 \%) \\
\text { Police: } 4(20 \%)\end{array}$ & $\begin{array}{l}\text { Got Desired Change } \\
\text { Hospital: } 5(25 \%) \\
\text { Police: } 1(5 \%) \\
\end{array}$ \\
\hline & No & $\begin{array}{l}\text { Immaterial } \\
\text { Hospital: } 1(5 \%) \\
\text { Police: } 15(75 \%)\end{array}$ & $\begin{array}{l}\text { Unanticipated Change } \\
\text { Hospital: } 1(5 \%) \\
\text { Police: } 0(0 \%)\end{array}$ \\
\hline
\end{tabular}

Table 34: Making Sense of Perceptions of Improved MHESU Engagement

The following distribution can be observed by looking at the table in Table 34. Seventeen respondents met the criteria for Thwarted Desires (65\% of Hospital sample, 20\% of Police sample), sixteen were classified as viewing the change as Immaterial (5\% of Hospital sample, $75 \%$ of Police sample), six met the criteria for Got Desired Change (25\% of Hospital sample, $5 \%$ of Police sample), and only one fit into the Unanticipated Change cell (5\% of Hospital sample, $0 \%$ of Police sample). Given that the majority of healthcare workers (65\% of Hospital sample) were classified in the Thwarted Desires cell of our typology, and the majority of police officers (75\% of Police sample) were classified in the Immaterial cell of our typology, we suspect that respondents from different TS stakeholders made sense of the planned TS change differently based on their perceptions of improved MHESU engagement. In the following subsections, we present data from respondents that we categorized as Got Desired Change and Thwarted Desires to help us understand how they made sense of the planned TS change.

Got Desired Change. Data analysis identified six respondents (25\% of Hospital sample, $5 \%$ of Police sample) as meeting our criteria for inclusion in the Got Desired Change cell of our making sense of the planned TS change typology (see Table 35). Our review of the post-change comments provided by these six respondents suggests that these employees interpreted the change in behaviors resulting from the planned TS change as an indication that there was increased 
collaboration between crisis workers and doctors. We labeled this first-order code to reflect what we heard: 'increased collaboration between crisis workers and doctors'. A police constable in this group, for example, made sense of these collaborative changes to the TS by making the following observations:

“The last time I was there, it seemed to, I don't know if they've changed how they do it on their end, it used to be we would sit in the back hall and wait until a room became available in emerge, at that point a crisis worker would come and see us and do the recommended stock and all that stuff, and then that was it for hours until the doctor came. But the last time I was there, they had all of the persons in crisis in the hallway and the crisis worker was back there taking person after person into a semi-private area where they could talk to them, and then clearly make recommendations to the doctor and the doctor came back there when he had time and signed off..."

\begin{tabular}{|c|c|c|c|c|c|c|}
\hline \multirow{2}{*}{$\begin{array}{c}\text { Got Desired Change: Improved MHESU } \\
\text { Engagement }\end{array}$} & \multicolumn{2}{|c|}{$\begin{array}{c}\text { Total } \\
(n=40)\end{array}$} & \multicolumn{2}{c|}{$\begin{array}{c}\text { Hospital } \\
(n=20)\end{array}$} & \multicolumn{2}{c|}{$\begin{array}{c}\text { Police } \\
\text { (n=20) }\end{array}$} \\
\cline { 2 - 7 } & $\#$ & $\%$ & $\#$ & $\%$ & $\#$ & $\%$ \\
\hline $\begin{array}{l}\text { Increased collaboration between crisis workers } \\
\text { and doctors }\end{array}$ & $\mathbf{6}$ & $\mathbf{1 5}$ & $\mathbf{5}$ & $\mathbf{2 5}$ & $\mathbf{1}$ & $\mathbf{5}$ \\
\hline $\begin{array}{r}\text { Increased collaboration between crisis workers and } \\
\text { doctors }\end{array}$ & 6 & 15 & 5 & 25 & 1 & 5 \\
\hline
\end{tabular}

Table 35: Got Desired Change: Improved MHESU Engagement

Thwarted Desires. As shown in Table 36, seventeen respondents (most of which were healthcare workers) in the panel data set were classified in the Thwarted Desires cell of our making sense of planned TS change typology. Analysis of the comments associated with improved MHESU engagement provided at Time 2 from these seventeen respondents resulted in the identification of three different first-order codes describing the ways these individuals made sense of the planned TS change. All three of these first-order codes were related to barriers within the 
MHESU that hindered changes within the TS.

\begin{tabular}{|c|c|c|c|c|c|c|}
\hline \multirow{2}{*}{$\begin{array}{c}\text { Thwarted Desires: Improved MHESU } \\
\text { Engagement }\end{array}$} & \multicolumn{2}{|c|}{$\begin{array}{l}\text { Total } \\
(n=40)\end{array}$} & \multicolumn{2}{|c|}{$\begin{array}{c}\text { Hospital } \\
(\mathbf{n}=\mathbf{2 0})\end{array}$} & \multicolumn{2}{|c|}{$\begin{array}{l}\text { Police } \\
(\mathbf{n}=\mathbf{2 0})\end{array}$} \\
\hline & \# & $\%$ & \# & $\%$ & \# & $\%$ \\
\hline MHESU Barriers to Changing PMI Process & 17 & 43 & 13 & 65 & 4 & 20 \\
\hline Psychiatrists unavailable to assess formed PMIs & 10 & 25 & 9 & 45 & 1 & 5 \\
\hline $\begin{array}{r}\text { Crisis workers not involved in PMI process in a } \\
\text { timely manner }\end{array}$ & 7 & 18 & 4 & 20 & 3 & 15 \\
\hline $\begin{array}{r}\text { MHESU was not discharging formed PMIs in a } \\
\text { timely manner }\end{array}$ & 4 & 10 & 4 & 20 & 0 & 0 \\
\hline
\end{tabular}

Table 36: Thwarted Desires: Improved MHESU Engagement

The respondents we group in the Thwarted Desires cell of our typology did not observe new behaviors related to increased engagement of MHESU staff in the PMI process, even though these behaviors were desired. When we asked respondents what they observed had changed within the TS at Time 2, respondents within the Thwarted Desires cell discussed three ways that the MHESU may be restraining changes to the PMI process: (1) the unavailability of psychiatrists, (2) the involvement of crisis workers in the PMI process, and (3) the rate at which the MHESU discharged PMIs. Details on each of these first-order codes are provided below.

Examination of these data suggests that nine healthcare workers and one police officer made sense of the planned TS change by thinking that the unavailability of psychiatrists prevented increased MHESU engagement. One Hospital security guard made the following comment, which we labeled: 'psychiatrists unavailable to assess formed PMIs'.

"It's good that we're getting these people through to the ED doctor, but ultimately I think most of the problem comes from not having a 24-hour psychiatrist on site. After hours we have a psychiatrist on call, but a large portion of the people that get put on a mental health form for various reasons they're all referred to psychiatry, but if there's no psychiatry here 
and they [PMIs] don't ultimately need to be here, then we are guarding them for an extended period of time that we don't need to be."

Four Hospital and three Police respondents that met our criteria for Thwarted Desires noted that, even though they wanted more engagement from crisis workers, they did not perceive any such changes. Based on the comments made by these seven respondents, it appears that they made sense of the planned TS change by thinking that the MHESU engagement did not increase because crisis workers did not increase their commitment to the process. We labeled comments of this nature: 'crisis workers not involved in PMI process in a timely manner'. A police constable discussed his observations post-change:

"I'm at a loss as to why the crisis workers aren't there full time with the amount of people they have coming in and out of there in these types of situations. The fact is that was a seven or eight hour wait to get a crisis [worker] there. And I think once again it's probably the case of the fact that the crisis workers know that, well, the man is there with the police, the police are going to keep him safe and keep him out of people's hair, again back to using the police and in this case the police in hospital as sort of a safe holding area type of thing."

Respondents we grouped in the Thwarted Desires cell of our typology made comments postchange that suggested MHESU staff were not discharging formed PMIs promptly, even though they made comments pre-change that they wanted this type of change to occur. According to the respondents' post-change comments, it appears that these four healthcare workers made sense of the planned TS change by thinking that the flow of PMIs through the PMI process did not speed up because PMIs were not discharged from the MHESU promptly. This type of comment was given the label: 'MHESU was not discharging formed PMIs in a timely manner'. One ED physician referenced an old I Love Lucy clip to describe his observations of the MHESU post- 
change:

"So we've improved the front end process right, but in terms of flow if you're not getting them out quickly, then you can't accept more. It's like the black and white video everyone shows of the woman doing the chocolate sorting... That's the classic that people always talk about right? So we're pushing the chocolates in faster but then we're not packaging as fast."

It should be noted that all thirteen of the healthcare workers in this cell worked in the ED, not the MHESU. In other words, ED workers made sense of the fact that desired changes had not been implemented by blaming a different group within the Hospital for this situation.

Our analysis of how respondents made sense of increased MHESU engagement can be summarized as follows:

- Healthcare workers in the "Desired Change" cell of our typology made sense of this change by focusing on cues supporting a positive outcome (i.e. greater collaboration between crisis workers and ED physicians).

- Healthcare workers in the "Thwarted Desired" cell of our typology made sense of this situation by focusing on how others (in this case MHESU) had prevented this change from being implemented.

\subsubsection{CASE FOUR: ANALYSIS OF PERCEPTIONS OF MANDATORY COMPLETION OF EDP FORMS}

The final case involves a planned Police change. This change was mandated by decisionmakers (see Section 5.1.2.3) to ensure that police officers completed EDP forms. Analysis of quantitative data (see Section 5.2) reveals that this planned change resulted in an increase to the

number of EDP forms completed by Police officers. Analysis of qualitative data (see Section 
5.3.1) provides evidence that some respondents have perceived police officers completing EDP forms more frequently after the implementation of the planned TS change than in the past. Accordingly, the purpose of this section is to get a better understanding of how Hospital and Police respondents made sense of the planned TS change by examining how respondents in the panel data set make sense of changes to ensure mandatory completion of EDP forms.

\begin{tabular}{|c|c|c|c|}
\hline & \multicolumn{2}{|c|}{$\begin{array}{c}\text { Perceived Police Officers Completing EDP Forms } \\
\text { (Time 2) }\end{array}$} \\
\hline & & No & Yes \\
\hline \multirow{3}{*}{$\begin{array}{c}\text { Desired } \\
\text { Completion } \\
\text { of EDP } \\
\text { Forms } \\
\text { (Time 1) }\end{array}$} & & Thwarted Desires & Got Desired Change \\
\hline & Yes & $\begin{array}{l}\text { Hospital: } 4(20 \%) \\
\text { Police: } 0(0 \%)\end{array}$ & $\begin{array}{l}\text { Hospital: } 0(0 \%) \\
\text { Police: } 0(0 \%)\end{array}$ \\
\hline & No & $\begin{array}{l}\text { Immaterial } \\
\\
\text { Hospital: } 15(75 \%) \\
\text { Police: } 6(30 \%)\end{array}$ & $\begin{array}{l}\text { Unanticipated Change } \\
\text { Hospital: } 1(5 \%) \\
\text { Police: } 14(70 \%)\end{array}$ \\
\hline
\end{tabular}

Table 37: Making Sense of Perceptions of Mandatory Completion of EDP Forms

We classified respondents into the four cells of our typology based on their responses to two of the interview questions: (1) whether or not the respondent mentioned they wanted police officers to complete EDP forms (before the introduction of the planned TS change), and (2) whether or not the respondent said that they perceived that police officers were completing EDP forms after the implementation of the planned TS change. This classification process divided the respondents into the four cells of our typology (Thwarted Desires, Unanticipated Change, or Immaterial with respect to their perceptions of the mandatory completion of EDP forms) as shown in Table 37.

The distribution of the respondents into the four cells is noteworthy. In particular, all four of the respondents who met the criteria for Thwarted Desires were healthcare workers $(20 \%$ of Hospital sample), whereas fourteen of the fifteen respondents who met the criteria for Unanticipated Change were police officers (5\% of Hospital sample, $70 \%$ of Police sample). Since none of the respondents stated in the Time 1 interviews that they wanted police officers completing 
EDP forms and commented in the Time 2 interviews that they perceived this change, none of our respondents could be considered as belonging to the Got Desired Change group. There was also fifteen Hospital and six Police respondents ( $75 \%$ of Hospital sample, $30 \%$ of Police sample) who met the Immaterial cell criteria. Taken as a whole, these data indicate that how a respondent made sense of this change depends on what TS stakeholder group they belong to. Below, we present our analysis of the data for the respondents in the Thwarted Desires and Unanticipated Change cells of our typology of making sense of planned TS change.

Thwarted Desires. Respondents that we grouped into the Thwarted Desires cell of our typology did not observe their desired changes associated with the completion of EDP forms Four respondents (all healthcare workers) met our criteria for inclusion in the Thwarted Desires cell of our typology (see Table 38). Analysis of the comments provided at Time 2 by these four healthcare respondents identified two first-order codes which reflect the ways in which these individuals made sense the situation in which they saw themselves (wanting a change that they had not felt was implemented). Both of these first-order codes speak to ways in which these healthcare workers perceived that the Police were creating barriers that were preventing this change from occurring: (1) dissonance between Police management and frontline officers, and (2) police officers were not completing EDP forms. Details on each of these two first-order codes are provided below. 


\begin{tabular}{|c|c|c|c|c|c|c|}
\hline \multirow{2}{*}{$\begin{array}{c}\text { Thwarted Desires: Mandatory } \\
\text { Completion of EDP Forms }\end{array}$} & \multicolumn{2}{|c|}{$\begin{array}{c}\text { Total } \\
(n=40)\end{array}$} & \multicolumn{2}{|c|}{$\begin{array}{c}\text { Hospital } \\
(\mathrm{n}=\mathbf{2 0})\end{array}$} & \multicolumn{2}{|c|}{$\begin{array}{l}\text { Police } \\
(n=20)\end{array}$} \\
\hline & $\#$ & $\%$ & $\#$ & $\%$ & \# & $\%$ \\
\hline Police Barriers to Changing PMI Process & 4 & 10 & 4 & 20 & $\mathbf{0}$ & $\mathbf{0}$ \\
\hline $\begin{array}{r}\text { Dissonance between Police management and } \\
\text { frontline officers }\end{array}$ & 3 & 8 & 3 & 15 & 0 & 0 \\
\hline Police were not completing EDP forms & 3 & 8 & 3 & 15 & 0 & 0 \\
\hline
\end{tabular}

Table 38: Thwarted Desires: Mandatory Completion of EDP Forms

Three of the healthcare workers in this cell made sense of their situation by attributing the inconsistency of completing EDP forms to a dissonance between Police management and frontline officers. We gave this type of comment the following first-order code: 'dissonance between Police management and frontline officers'. One MHESU nurse discussed her sensemaking of the planned TS change by saying:

"As for the [EDP] form, this is the thing with any kind of change...you may have buy in from Police administration but when it comes to the actual implementation by the people who do the job then that's a whole different ball game."

These same three healthcare workers also appear to have made sense of the planned TS change by thinking that frontline police officers were not filling EDP forms out, which would have led to inconsistencies associated with the completion of EDP forms. This type of comment was given the label: 'Police are not completing EDP forms'. A MHESU manager discussed her observations of the planned TS change:

"I don't think the completion rate of the EDP forms has gotten better. The first step is to get the police to fill them out, then our team in the ED or maybe a crisis worker could take over."

Unanticipated Change. Analysis of the comments provided at Time 2 by respondents that we classified into the Unanticipated Change cell of our typology (all of whom are police 
officers) are shown in Table 39 and discussed below. We identified two first-order codes that reflected the ways that these 12 police officers made sense of changes within the TS that required them to complete EDP forms - a change that they had not expressed any opinion on pre-change. Both of these first-order codes speak to the idea that these Police officers equate changes to the EDP form protocol to the idea that the act of filling out the form strained Police resources.

\begin{tabular}{|c|c|c|c|c|c|c|}
\hline \multirow{2}{*}{$\begin{array}{l}\text { Unanticipated Change: Mandatory } \\
\text { Completion of EDP Forms }\end{array}$} & \multicolumn{2}{|c|}{$\begin{array}{c}\text { Total } \\
(\mathrm{n}=\mathbf{4 0})\end{array}$} & \multicolumn{2}{|c|}{$\begin{array}{c}\text { Hospital } \\
(\mathbf{n}=\mathbf{2 0})\end{array}$} & \multicolumn{2}{|c|}{$\begin{array}{l}\text { Police } \\
(\mathrm{n}=\mathbf{2 0})\end{array}$} \\
\hline & $\#$ & $\%$ & \# & $\%$ & $\#$ & $\%$ \\
\hline Police Resources Strained & 12 & 30 & 0 & 0 & 12 & 60 \\
\hline $\begin{array}{r}\text { Frontline officers spend more time completing } \\
\text { paperwork }\end{array}$ & 11 & 28 & 0 & 0 & 11 & 55 \\
\hline $\begin{array}{r}\text { Frontline officers spend more time tracking down } \\
\text { sergeants to sign EDP form }\end{array}$ & 7 & 18 & 0 & 0 & 7 & 35 \\
\hline
\end{tabular}

Table 39: Unanticipated Change: Mandatory Completion of EDP Forms

Analysis of the data identified three ways that Police resources became more strained: (1) frontline officers spend more time completing paperwork, (2) frontline officers spend more time tracking down sergeants to sign EDP form, and (3) frontline officers make copy of EDP form for Hospital records. Details on each of these responses are given below.

Just over half of the Police respondents (55\% of Police sample) talked about how the requirement to fill out the form reduced the number of police officers available to take service calls in the community. They blamed this on the fact that more frontline officers were spending time completing paperwork associated with the EDP form. In other words, officers in this cell made sense of the planned TS change by thinking it required frontline officers to spend more time completing paperwork than "real police work". This type of comment was given the following label: 'Frontline officers spend more time completing paperwork'. For example, two police constables made the following observations. 
"I think some members may find the additional paperwork, like the EDP form, a little bit much. Even though we put on a report, we do notes, we have all this other stuff going on and this is one additional piece of paper that we've got to fill out for the Hospital, and I think a lot of members are like, are you kidding me? Adding another cog in the wheel here, something else we need to do..."

“That EDP form, I mean it's, it's trivial in nature but it's... you have to fill it out at the hospital then you have to get it signed... you have to leave the carbon copy for the hospital, you have to bring the white copy back to the division, have it signed by a supervisor, now they want you to scan it and upload it to a public drive that all personnel can access and then submit it to the mental health coordinator. There's a lot redundant steps in there that I don't think need to be there... You want the EDP form at the hospital that's fine... but we're always covered off by the occurrence, so the EDP form is to me redundant in the first place because everything that's on the EDP form is addressed in the occurrence which is always there, and now we've got this paper copy, like a hard copy, that has to be lodged somewhere, it's really like going back in time. Everybody is going paperless and we're creating more paper."

Seven police officers made comments that suggested they made sense of the planned TS change by thinking that Police resources were strained because frontline officers were spending less time on the road and more time tracking down sergeants. More specifically, these respondents commented that frontline officers were spending more time looking for and meeting with their sergeant so that they could get the EDP form appropriately signed off. This type of comment was given the first-order code: 'frontline officers track down sergeants to sign EDP form'. For example, one Police constable discussed his observations post-change by stating: 
"They [EDP forms] are being completed now because you have to have them signed by a supervisor after, and it has to be indicated on the reports, so, yes they are being filled out. Are supervisors always easy to get a hold of? No."

Our analysis of how respondents made sense of mandatory completion of EDP forms can be summarized as follows:

- Healthcare workers in the "Thwarted Desired" cell of our typology made sense of this situation by focusing on how others (in this case the police) had prevented a change that they wanted to be implemented.

- Police officers in the Unanticipated Change cell of the typology made sense of this unexpected change by focusing on how the change had negatively impacted their TS stakeholder (i.e. strained police resources).

\subsubsection{EMPIRICAL ASSESSMENT OF MAKING SENSE OF PLANNED TS CHANGE: CROSS-CASE ANALYSIS}

In Section 5.4.4, we presented a within-case analysis of four different cases. This section presents a cross-case analysis of the four cases and, in so doing, develops our understanding of how individuals make sense of a planned TS change. We conducted the cross-case analysis by comparing second-order categories within a particular quadrant across each of the four cases. We grouped similar second-order categories to form third-order categories within that cell. Key observations with respect to the sensemaking used by the respondents in each of the cells of our typology are shown in Table 40 and summarized in the following three subsections. 


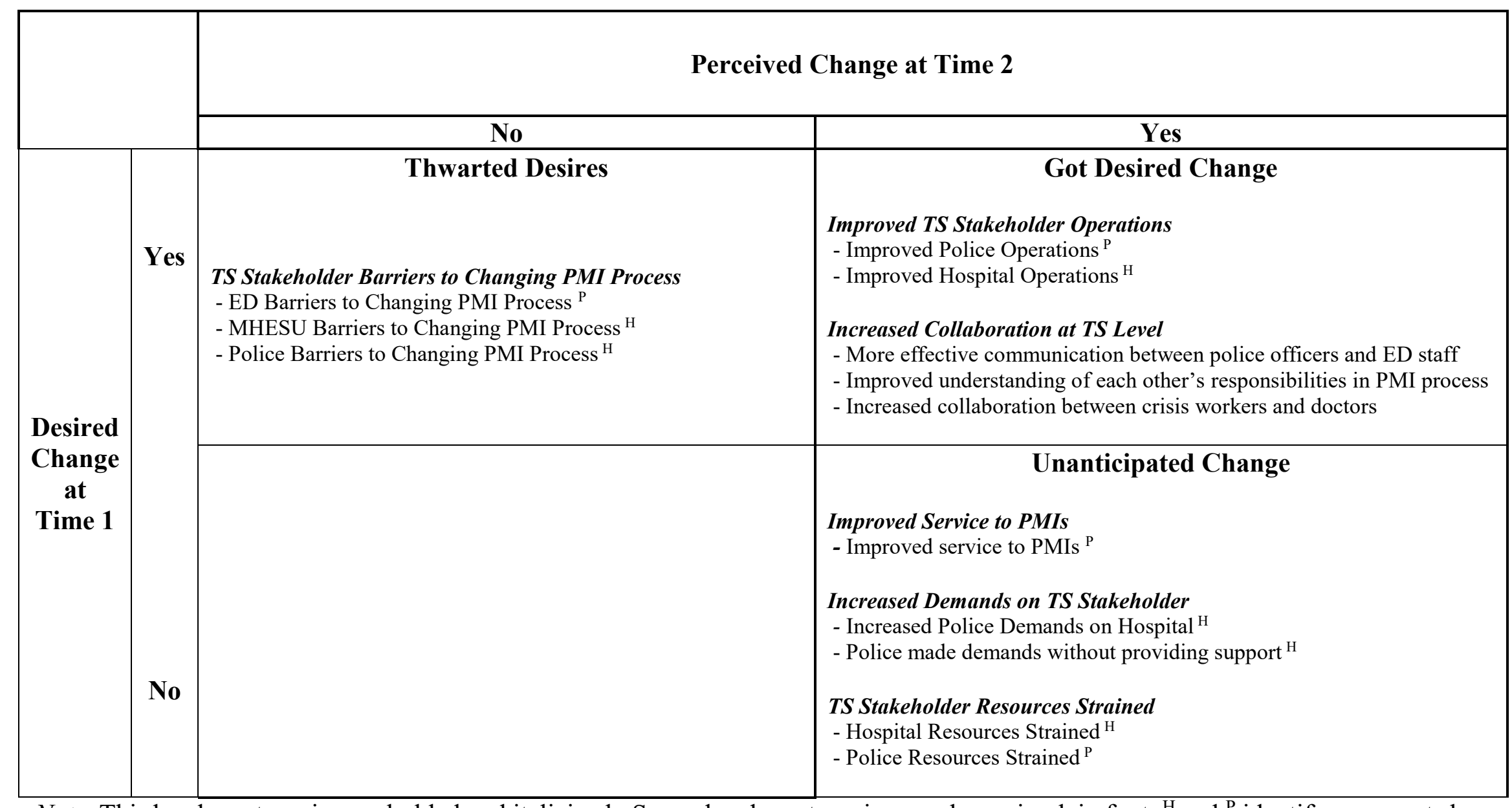

Note. Third-order categories are bolded and italicized. Second-order categories are shown in plain font. ${ }^{\mathrm{H}}$ and ${ }^{\mathrm{P}}$ identify comments by predominantly healthcare workers and police officers (e.g. at least $20 \%$ more healthcare workers than police officers), respectively.

Table 40: Making Sense of Planned TS change 


\subsubsection{GOT DESIRED CHANGE}

We analyzed how the respondents in the Got Desired Change cell of our typology made sense of the planned TS change by considering how they perceived four specific changes (i.e., increased interorganizational communication, an ED process that expedites service for PMIs, improved MHESU engagement, and mandatory completion of EDP forms). Comparison of the second-order categories describing the sensemaking of those in the Got Desired Change cell across each of the four cases resulted in the creation of two third-order categories: (1) improved TS stakeholder operations, and (2) increased collaboration at the TS level. Our analysis also indicates that comments made about improvements to Hospital operations were predominantly made by healthcare workers, whereas comments made about improvements to Police operations were predominantly made by police officers. Interestingly, this analysis suggests that employees in this cell of our typology make sense of the planned TS change by thinking that the new behaviors benefited their TS stakeholder as well as the TS.

Sensemaking theory can partially explain these findings. Sensemaking triggers can contribute to the development of new schemata, as the goals of the interventions, the interventions themselves, and organizational flaws (that limit the effectiveness of the interventions) may impact how individuals make sense of their environment (Balogun \& Johnson, 2006). Balogun and Johnson (2006), for example, found that when the goals of an intervention are not aligned with that of an individual, the individual's sensemaking process led to intergroup tensions and barriers. When the goals of an intervention are consistent with that of an individual, however, the individual's sensemaking process led to positive staff attitudes and staff adaption (Balogun \& Johnson, 2006). Balogun and Johnson's (2006) findings are consistent with those of this thesis, which show respondents who perceived changes that they wanted (i.e. desired change at Time 1, 
perceived change at Time 2) made sense of the changes as something that positively impacted TS stakeholders.

Balogun and Johnson's (2005) sensemaking theory does not, however, explain why healthcare workers and police officers tended to comment on the ways that their own TS stakeholder benefitted from the implementation of the change. To address this finding, we turn to attribution theory. In Harvey et al.'s (2012) meta-analysis of attribution theory in organizational sciences, they find that individuals associate favorable outcomes with internal attributions more than external attributions. If we were to assume that getting a desired change was a favorable outcome, then attribution theory would explain why respondents in this cell make sense of getting the desired changes they want by thinking that their behavior within the TS was associated with getting the change they wanted.

It should also be noted that this cross-case analysis shows that respondents from both the Hospital and the Police made sense of getting the changes that they desired by thinking there was greater collaboration at the system level. It is possible that the sensemaking processes of individuals in this cell were influenced by sensegiving, which refers to the notion that people may shape how an individual makes sense of a change (Maitlis \& Lawrence, 2007). As the overarching objective of the pilot project was to develop the interorganizational relationships, it is likely that decision-makers communicated this objective to their teams (Balogun, 2006). This form of sensegiving may have shaped how individuals were making sense of the change. However, as we have little information about organizational communication, we can not confirm that this is the case. Future research may benefit from exploring how organizational communication and organizational sensegiving may shape how individuals make sense of planned TS change.

\subsubsection{THWARTED DESIRES}


We then investigated how respondents who met our criteria for Thwarted Desires made sense of the planned TS change by examining the second-order categories we identified for each of the four cases. Our cross-case analysis indicated that all three of these second-order codes could be grouped within a single third-order category: TS stakeholder barriers to changing the PMI process. Additionally, the cross-case analysis suggests that respondents make sense of not getting their desired change by blaming their TS partner for this situation (i.e. police officers talked about barriers to change within the ED, while healthcare workers focused on barriers to change within the Police).

Sensemaking theory can partially explain these findings. Balogun (2006) has argued that employees may not recognize that 'things have changed' when sensemaking triggers (i.e., change interventions, flaws in the organization) are ineffective at challenging their old schemata. Our analysis showed that respondents felt barriers within the Hospital and Police were preventing desired changes from materializing. According to Balogun (2006), this would suggest that the change interventions did not trigger cues that disrupted existing schemata concerning the TS.

Balogun's (2006) sensemaking theory does not, however, explain why healthcare workers and police officers tended to speak about the ways in which their partners were hindering the implementation of a change that they desire. We again turn to attribution theory to get a better understanding why one group of respondents might 'blame' another group for preventing a desired change from occurring. Harvey et al. (2012) find that individuals associate unfavorable outcomes with external attributions more than internal attributions. If we were to assume that 'thwarted' desires were unfavorable outcomes, then attribution theory would explain why respondents in this cell make sense of this type of situation (i.e. one where they did not perceive a change that they 
wanted) by attributing the unfavorable outcome to an external factor (i.e. in this case their TS partner).

\subsubsection{UNANTICIPATED CHANGE}

Lastly, we analyzed the sensemaking about planned TS change for those respondents who met our criteria for Unanticipated Change by examining their comments about each of the four specific changes (i.e. cases). Our cross-case analysis of the five second-order categories describing how the respondents in this cell made sense of this situation resulted in the formation of three thirdorder categories: (1) improved service to PMIs, (2) increased demands on TS stakeholder, and (3) TS stakeholder resources strained.

A follow-up analysis of the data provided by respondents in the Unanticipated Change cell of our typology was conducted to help us better understand how the two TS stakeholders in this phase of our analysis made sense of Unanticipated Change. Examination of the data showed that the TS stakeholder who was required to make the Unanticipated Change generally made sense of the planned TS change by focusing on how the change had made things worse for them. On the other hand, respondents who observed an Unanticipated Change to the behaviour of their TS partner made sense of the planned TS change by focusing on how the change had improved things

for them. For example, healthcare respondents made sense of the Unanticipated Change of creating an ED process to expedite PMIs and Police by thinking that their resources were strained (40\% of Hospital sample, $0 \%$ of Police sample) and that they had more demands from Police (20\% of Hospital sample, $0 \%$ of Police sample), while police officers made sense of the Unanticipated Change associated with the mandatory completion of the EDP form by thinking that their resources were strained ( $0 \%$ of Hospital sample, $60 \%$ of Police sample). 
Perhaps the most thought-provoking example of respondents discussing one TS stakeholder changing for the benefit of the other relates to respondents' sensemaking of increased communication between TS stakeholders (i.e. the first case). While we coded this perception of change as a system level change (because multiple TS stakeholders needed to be involved for this outcome to occur), the majority of the effort to change the communication came from the Hospital, as healthcare workers began sharing information with their partners more promptly than they did prior to the implementation of the planned TS change. Our analysis of these data revealed that, while all eight police officers in this cell thought that this change was for the better as it improved the situation for PMIs, ten of the twelve healthcare workers in this cell interpreted this Unanticipated Change as something that changed their situation in a negative manner given that Police were placing more demands on them.

These findings are interesting, as they suggest that how respondents make sense of unanticipated aspects of a planned TS change will vary depending on whether or not the change under consideration requires them to change their own behaviour. More specifically, employees make sense of an Unanticipated Change that requires them to make behavioral changes by focusing on the costs to their TS stakeholder of making this change (e.g., strained organizational resources, additional organizational demands). Alternatively, employees will make sense of Unanticipated Change that necessitates behavioral changes from their TS partner by concentrating on how such a change with benefit themselves or others (e.g. improved service to PMIs). Social exchange theory may partially explain why this is occurring. Social exchange theory posits that relationships are formed by the use of a subjective cost-benefit analysis (Cook, Cheshire, \& Rice, 2013) and implies a two-sided, mutually contingent, and rewarding process involving transactions. Costs refer to the elements of relational life that have negative value to a person, such as the effort put into a 
particular activity (Baxter \& Braithwaite, 2008), while benefits refer to the elements of a relationship that have a positive value, such as what is received in an exchange (Monge \& Contractor, 2003). According to social exchange theory, costs outweighing the benefits reflect a negative exchange relationship, while benefits outweighing the costs reflect a positive exchange relationship (Cook et al., 2013).

In this case study, we note that the planned TS change involved a number of different changes. Examination of these various changes suggests an exchange relationship whereby the Hospital implemented planned changes to benefit the Police (i.e. shorter wait times) and the Police implemented planned changes to benefit the Hospital (i.e. mandated completion of EDP forms). Thus, social exchange theory may explain why respondents made sense of Unanticipated Changes that required behavioral changes from their TS stakeholder by focusing on the costs of making this change, and why respondents made sense of Unanticipated Changes that required behavioral changes from their TS partner by focusing on the benefits from their efforts.

\subsection{DISCUSSION: PLANNED TS CHANGE}

This chapter began by providing the reader with a summary of the events leading up to the planned TS change (Section 5.1). We then examined four quantitative indicators to get an appreciation of whether or not the planned changes introduced into the TS had a quantifiable impact on the TS (Section 5.2). We also reviewed what respondents wanted to change within the TS, as well as how they thought the TS was perceived to have changed (Section 5.3.1), and how respondents made sense of the planned TS change (Section 5.3.2). Five summary points can be made from the analysis undertaken in this chapter.

- Management actions impacted how change unfolded in the TS over time. 
- Pre-change: few respondents felt that the TS stakeholders they were a part of needed to change their behavior within the TS. Only healthcare workers expressed the need for change in their own organization.

- Pre-change: all stakeholders felt that their TS partners should change.

- Post-change: respondents did not report perceiving many of the changes that their partners felt had occurred.

- Findings indicate that in some cases TS stakeholders felt that the TS had changed in ways that they wanted (i.e. Got Desired Change) while in other cases, they felt the changes that they desired had not been implemented (i.e. Thwarted Desires). All three TS stakeholders reported that they perceived Unanticipated Changes. This would support the idea that when it comes to planned TS change, the TS stakeholder "can't always get what they want".

- Findings indicate that there are four possible ways that individuals within a TS can make sense of a planned TS change.

In the subsections below we address each of these summary points.

\subsubsection{MANAGEMENT ACTIONS IMPACTED HOW CHANGE UNFOLDED IN THE TS} OVER TIME

Consistent with much of the literature on planned change (e.g. Burnes, 1996), this research finds that the actions of senior managers impacted what happened within their organizations (Orlikowski, 1996) as well as their TS partners (de Hond \& den Bakker, 2007). We found that the managerial act of the decision-makers implementing four specific changes (see analysis in Section 5.1) impacted actual changes within the TS (see analysis in Section 5.2), which further impacted respondents' perceptions of what changed within the TS over time (see analysis in Section 5.3). For example, in July 2014, managers launched the Intervention and Escalation Policy, which was 
designed to expedite PMIs who were brought in to the Hospital by police officers. Immediately after the implementation of this new policy, the amount of time police officers had to wait for their detained PMI to be assessed by an ED physician substantially decreased (e.g. average wait time in June was 2.43 hours, while average wait time in July was 1.83 hours). When we interviewed respondents six months after the implementation of this policy, the majority of healthcare and police respondents also mentioned that there had been a substantial decrease in the average length of time police officers had to wait in the Hospital. These findings support the notion that the outcomes of planned changes can be known in advance (Olikowski, 1996), as in this case decisionmakers within the TS implemented a set of changes that achieved two of their three desired objectives.

\subsubsection{DESIRED CHANGES WITHIN THE TS}

Before the implementation of planned TS change respondents from only one of the three TS stakeholders (i.e. Hospital) mentioned that they felt that they personally needed to make changes to how they operated within the TS. By comparison, in the Time 1 interviews, respondents from all three TS stakeholders mentioned that they wanted their TS partners to change how they did things.

The attribution and organizational silence literature may help us understand why this might be happening. Harvey et al.'s (2012) meta-analysis of attribution research is consistent with research on self-serving attributional biases, which suggests that people often feel better when they attribute blame for problematic outcomes to external factors and take credit for favorable outcomes. If we were to assume that individuals who wanted a particular TS change were unhappy with the status quo, then self serving bias would explain why such individuals are more likely to 
want their partner to change their behaviour in a way that improves the situation than they are to see that they personally need to change.

A second perspective, organizational silence refers to "powerful forces in organizations that cause widespread withholding of information about potential problems or issues by employees" (Morrison \& Milliken, 2000, p.706). Morrison and Milliken (2000) theorize that organizational silence can lead to less effective organizational change processes (i.e. a barrier to organizational change) given that silence may involve: (1) a lack of variance in informational input, (2) lack of critical analysis of ideas and alternatives, and (3) lack of negative internal feedback. The fact that our analysis indicates all respondents primarily identified ways in which their TS partner needed to change suggests that respondents may be displaying a form of organizational silence within the context of a TS in that they do not engage in critical analysis with respect to their own behaviour within the TS and focus instead on what their partners should do rather than what changes they personally need to make.

This finding is interesting in that, while respondents are vocal about what needs to change in their TS partner, they are silent about what needs to change in their TS stakeholder. This type of organizational silence was not identified by Morrison and Milliken (2000) in their original conceptualization of concept. Morrison and Milliken (2000) theorize that organizational silence may stem from implicit managerial beliefs (i.e., belief that employees are self-interested, belief that management knows best, belief that unity is good and dissent is bad) and fear of managers' negative feedback. Future research may benefit from exploring the impact of individuals being vocal about their partners and silent about their TS stakeholder in the context of planned TS change. 


\subsubsection{PERCEIVED CHANGES WITHIN THE TS}

Few of the respondents mentioned that they observed a change taking place in their TS partners, even though respondents from all TS stakeholders noted changes had occurred in their own TS stakeholder. Weick et al. (2005) contend that sensemaking is retrospective and not necessarily accurate. Weick (1995) also argues that sensemaking involves individuals comparing their preconceived notions of behavior within one organization with more recent observations of behaviors within that organization to identify environmental discrepancies that might lead them to a better understanding of how the organization might have changed. This logic would suggest that an individual's ability to recognize changes within one organization is dependent on the individual's experiences with that particular organization. As healthcare workers are likely to have an entirely different set of experiences than that of a police officer or support worker in the community, individuals working in one profession are also likely to identify different environmental discrepancies than individuals working in other occupations. This may have led, for example, police officers to identifying differences with how EDP forms were completed after changes were implemented, while healthcare or social workers did not.

Another rationale for respondents not mentioning that they observed changes taking place in their partner may be that the changes that have taken place had not yet impacted them in their organization. Change theorists (e.g. Pettigrew et al., 2001; Langley et al., 2013) have noted that change initiatives often require more time than expected to create substantial impact. Future research may benefit from exploring TS change over three or more time periods to investigate whether a TS stakeholder's change takes longer to impact their partner than other changes. 
Despite the theory behind each of these arguments, we do not know why respondents are less aware of changes taking place in their TS partner than in their TS stakeholder. Future research may benefit from delving deeper into this area of research.

\subsubsection{YOU CAN'T ALWAYS GET WHAT YOU WANT}

Our analysis of the cross-sectional data suggests that the changes that were introduced within the TS differentially benefited certain TS stakeholders. More specifically, the Hospital and Police perceived a change that they wanted (i.e. Got Desired Change), the Hospital and the Social Service Agency wanted a change but did not perceive that it had occurred (i.e. Thwarted Desires), and the Hospital, Police, and Social Service Agency perceived a change in the TS that they did not mention they wanted (i.e. Unanticipated Change). The fact that TS stakeholders varied in the extent that they benefited (or didn't benefit) from the planned TS change is consistent with TS change literature. As certain TS stakeholders are more capable of achieving the changes that they want within a TS (Ahuja et al., 2012), it is not surprising that some of the TS stakeholders got what they desired while others did not.

It is interesting to note that respondents from all three TS stakeholders thought that their organization had to change in ways that they had not unanticipated prior to the introduction of the planned TS change. This finding would suggest that: (1) respondents did not want the changes in the TS that were implemented by decision-makers, and/or (2) the planned TS change resulted in unexpected outcomes (i.e. planned $1^{\text {st }}$ order change results in unanticipated second order changes). Future research may benefit from examining the relationship between frontline employees not wanting changes that their managers wanted and unexpected outcomes from a planned TS change.

\subsubsection{MAKING SENSE OF PLANNED TS CHANGE}


Empirical data collected for this thesis is supportive of our theoretically derived $2 \times 2$ typology which hypothesizes that individuals' sensemaking about a planned TS change depends on whether or not they wanted a particular change at Time 1 (i.e. prior to the change being introduced) and whether or not they observed that a particular change in the TS after changes had been introduced (i.e. at Time 2). In this thesis, we examined situations illustrating each of the four different ways respondents make sense of the planned TS change that are included in our typology. From a sensemaking perspective, individuals belonging to different quadrants of this typology seemed to be making sense of the planned TS change in very different ways, as they were attaching meaningful explanations to their perceptions, regardless of their perception's accuracy (Weick, 1995).

The fact that we were able to identify four different ways that respondents made sense of the planned TS change may be essential to understanding how change unfolds within a TS (or elsewhere) over time. In recent research, Kim et al. (2006) have argued that interorganizational inertia may impede TS change. They have also argued that there is less inertia when partnerships have gone through earlier changes, as those earlier changes may have built structures to facilitate future change (ibid). However, Kim et al.'s (2006) research does not consider (nor did it intend to) that employees can interpret TS changes differently. While past TS changes that have been interpreted as positive may lead to future changes, past changes that have been interpreted as failures or negative may limit or even doom the efforts of future change (Wanous, Reichers, \& Austin, 2000). This finding suggests that future TS change research should consider how employees make sense of previous TS changes when investigating interorganizational inertia.

This typology also offers promising avenues for future studies as it identifies four different ways that employees may make sense of a change. Weick (1995) also argues that sensemaking is 
ongoing and iterative, and thus may change over time. Future researchers may benefit from tracking how an individual may "move" within the typology over multiple time periods. For example, does a pattern emerge suggesting that Thwarted Desires become Got Desired Change over time? What is the role of sensegiving in this transition? Moreover, how do organizational dynamics change once that transition occurs?

The findings associated with this typology are consistent with the notion that "a change" is multi-faceted and includes both planned as well as emergent components (Bamford \& Forrester, 2003). For example, the planned TS change had planned (e.g. mental health training) and emergent (e.g. increased interorganizational communication) components. These findings also suggest that individuals within different organizations can make sense of these different components of change (i.e. planned and emergent) in very different ways. Moreover, it would seem that the focus tends to be egocentric (how can or did my organization benefit) rather than altruistic (how can we change to benefit the TS or our partners within the TS). Future research may benefit from exploring the sensemaking of planned and emergent changes in order to understand the causality of emergent changes. While this research applies this typology at the transorganizational level, future research may also benefit from examining this typology at the organizational level.

These findings suggest that respondents' sensemaking of the planned TS change may impact perceptions of TS stakeholder relationships over time. While the results from this chapter imply that some respondents made sense of the planned TS change by thinking that their TS stakeholder benefitted from the change, which might strengthen interorganizational relationships, other respondents made sense of the planned TS change by thinking that their TS stakeholder was harmed by the change, which might weaken interorganizational relationships. To get a better understanding of how the planned TS change may have impacted perceptions of TS stakeholder 
relationships, we explore the stakeholder perspective to interorganizational relationships within the next chapter. 


\section{CHAPTER 6: ANALYSIS OF THE RESULTS THROUGH A STAKEHOLDER THEORY LENS}

This chapter will examine the results of data analysis relating to the perceptions of TS stakeholder relationships at two time periods: leading up to the planned TS change (i.e. Time 1) and six months after TS changes were implemented (i.e. Time 2). In this thesis, we conceptualize the TS stakeholder relationship construct including the three stakeholder dimensions identified by Mitchell et al. (1997): power, legitimacy, and urgency. The analysis reported in this chapter is guided by the following research question:

How do TS stakeholders' perceptions of (a) power, (b) legitimacy, and (c) urgency within the TS before the implementation of planned change compare to their perceptions of (a) power, (b) legitimacy, and (c) urgency six months after the implementation of planned TS change?

This chapter is structured as follows. We first present data on perceptions of power (Section 6.1), legitimacy (Section 6.2), and urgency (Section 6.3) within a TS. The presentation of these analyses is followed by a discussion of TS stakeholder relationships in a TS undergoing a planned change in Section 6.4.

\subsection{PERCEPTIONS OF POWER WITHIN THE TS}

The presentation of findings associated with perceptions of TS stakeholder power is broken down as follows. The first four sections present the results of the perceptions of power within the TS based on the cross-sectional data set. More specifically, we first present data reflecting perceptions of power within the TS before the planned TS change was implemented. Findings using the total sample are presented first in Section 6.1.1. This is followed in Section 6.1.2 by an examination of the findings at the level of the TS stakeholder. We then present perceptions of power within the TS after the implementation of the planned TS change. Section 6.1.3 presents findings from the total sample, whereas Section 6.1.4 presents an examination of these post-change 
findings at the level of the TS stakeholder. The final section, Section 6.1.5, explores how the planned TS change may have impacted perceptions of TS stakeholder power over time by examining both cross-sectional and panel data regarding perceptions of TS stakeholder power over time.

\subsubsection{PERCEPTIONS OF POWER WITHIN THE TS: TOTAL SAMPLE PRE-CHANGE}

\section{(TIME 1)}

The five first-order codes emerging from the analysis of the first interview question (see Section 4.5.2) are displayed in Table 41, along with the three second-order categories (shaded) to which they are associated. Of the five forms of perceived power mentioned by employees within the TS, three describe the perceived power displayed by the Hospital in the TS, one describes the perceived power displayed by the Police in the TS, and one describes the perceived power demonstrated by the Social Service Agency in the TS. The one type of power that was perceived to be held by the Police was mentioned by less than $10 \%$ of the total sample and will not be discussed in this section.

\begin{tabular}{|c|c|c|}
\hline \multirow{2}{*}{$\begin{array}{c}\text { Perceptions of Power in the TS: Total } \\
\text { Sample Pre-Change (Time 1) }\end{array}$} & \multicolumn{2}{|c|}{$\begin{array}{c}\text { Total } \\
(n=75)\end{array}$} \\
\hline & \# & $\%$ \\
\hline Perceived Power Displayed by Hospital & 70 & 93 \\
\hline Doctor decides PMI's treatment & 44 & 59 \\
\hline Hospital staff decides when police officers can leave & 30 & 40 \\
\hline Doctor decides whether PMI is formed & 21 & 28 \\
\hline Perceived Power Displayed by Police & 6 & 8 \\
\hline Police presence influences PMI process flow & 6 & 8 \\
\hline $\begin{array}{l}\text { Perceived Power Displayed by Social Service } \\
\text { Agency }\end{array}$ & 10 & 13 \\
\hline Social worker requests Police assistance & 10 & 13 \\
\hline
\end{tabular}

Table 41: Perceptions of Power within the TS: Total Sample Pre-Change (Time 1) 
Second-order categories: When we asked employees how power was displayed in the TS before changes were implemented, examination of the second-order categories support the following observations. Nearly all respondents discussed ways the Hospital displayed power ( $93 \%$ of total sample) in the TS. Few respondents indicated that either the Police ( $8 \%$ of total sample) or the Social Service Agency (13\% of total sample) demonstrated power in the TS. Analysis of the first-order codes helps us understand why this might be the case.

Perceived Power Displayed by Hospital: According to the employees we interviewed, the Hospital displays power in the following three ways: (1) by the doctor deciding the PMI's treatment, (2) by the Hospital staff deciding when police officers can leave, and (3) by the doctor deciding whether the PMI is formed. The most commonly cited way that the Hospital displays power within the TS was given the descriptor 'Doctor decides PMI's treatment'. The fact that nearly two-thirds of the respondents felt that the Hospital displayed power in this way suggests that the doctor's decision related to the treatment of the PMI greatly impacts the PMI process. The following quotes illustrate this type of power perceived to be displayed by the Hospital:

"Once triaged, they will be moved to an assessment unit for the doctor and from there they will be put in whatever mental health unit is best suited for their needs." (Hospital Administrator)

"At times the Hospital won't even take them, or give them anything, and it's clear they need meds..." (Police Constable)

"They [ED physicians] can change the medication the client takes... it can change the plan we need to follow." (Social Service Agency Frontline ACT)

The second most common kind of power respondents perceived to be displayed by the Hospital is labelled 'Hospital staff decides when police officers can leave'. Forty percent of the 
total sample made comments that were related to the idea that the staff at the Hospital could determine when they assessed the PMI and, as a result, could determine when police officers were allowed to leave the Hospital. For example:

"It depends on the staff who are on but definitely the staff are in control of when the police will get to speak with the physician or with their patient and how long they will wait there. So really the hospital is in control of the PMI process." (Hospital ED Nurse)

"I don't know if you'd say the administrative side or the physician side has the ultimate power because that poor patient and that poor officer are sitting there waiting for the physician. At the end of the day, he's got the power to make the decision: yes, I'm going to see this person now, or no, it's not important enough, and I'm going to move on." (Police Constable)

"We waited there, the police changed hands twice while we were there. And then, we waited more and then the nurse told us we could see the doctor." (Social Service Agency Frontline $\mathrm{ACT})$

Approximately a third of respondents discussed the doctor's ability to form PMIs when asked about power within the TS. We labelled this type of comment 'Doctor decides whether PMI is formed'. Forming a PMI means that the PMI has their liberties suspended (i.e. must stay at Hospital) and that a psychiatrist must assess this individual within 72 hours. The following quotes illustrate this form of perceived power:

"I mean we have the power to keep them for 72 hours... so that's certainly physician power and power from the organisation as a whole. We can decide whether we're going to keep you or whether we're going to let you go." (Hospital Psychiatrist) 
"The hospital does have the power to issue a form on this patient to keep them on site for the next 72 hours for further site assessment." (Police Constable) "If the psychiatrist that does the assessment finds that the client is formable, that is, if they're at risk to themselves or others, they can detain them for 72 hours. Their liberties are suspended for 72 hours." (Social Service Agency Frontline Justice)

Perceived Power Displayed by Social Service Agency: Analysis of the data suggests that the only type of power that the Social Service Agency was perceived to display within the PMI process at Time 1 is associated with social workers filling out a form to initiate Police action. We labelled this type of perceived power: 'Social worker requests Police assistance'. Ten out of the 75 respondents made comments that were similar to the following comment by a social worker: "We'll complete the Form 2, and reasons why we believe the client needs to be hospitalised, and at that point either the Justice of the Peace will agree or not, and then we'll take the documentation to the police station in order to inform the police, and then the police will apprehend the client and take them to the Hospital." (Social Service Agency Frontline Justice)

\subsubsection{PERCEPTIONS OF POWER WITHIN THE TS: TS STAKEHOLDER SAMPLE PRE-CHANGE (TIME 1)}

Table 42 shows the responses given to questions associated with perceptions of power within the TS at Time 1 broken down by TS stakeholder. Analysis of second-order categories are reported first, followed by the analysis of first-order categories. 


\begin{tabular}{|c|c|c|c|c|c|c|c|}
\hline \multirow{2}{*}{$\begin{array}{l}\text { Perceptions of Power within the TS: TS } \\
\text { Stakeholder Sample Pre-Change (Time } \\
\text { 1) }\end{array}$} & \multicolumn{2}{|c|}{$\begin{array}{c}\text { Hospital } \\
(\mathbf{n}=\mathbf{2 5})\end{array}$} & \multicolumn{2}{|c|}{$\begin{array}{l}\text { Police } \\
(\mathbf{n}=\mathbf{2 5})\end{array}$} & \multicolumn{2}{|c|}{$\begin{array}{l}\text { Social Service Agency } \\
\qquad(\mathbf{n}=\mathbf{2 5})\end{array}$} & \multirow{2}{*}{$\begin{array}{c}\text { Pre-Change Perception } \\
\text { (Common / Shared / } \\
\text { Unique) }\end{array}$} \\
\hline & \# & $\%$ & $\#$ & $\%$ & \# & $\%$ & \\
\hline Perceived Power Displayed by Hospital & 24 & 96 & 24 & 96 & 22 & 88 & Strong Common \\
\hline Doctor decides PMI's treatment & 18 & 72 & 15 & 60 & 11 & 44 & Strong Shared $(\mathrm{H}, \mathrm{P})$ \\
\hline Hospital staff decides when police officers can leave & 9 & 36 & 13 & 52 & 8 & 32 & Strong Common \\
\hline Doctor decides whether PMI is formed & 6 & 24 & 4 & 16 & 11 & 44 & Weak Common \\
\hline Perceived Power Displayed by Police & 6 & 24 & $\mathbf{0}$ & $\mathbf{0}$ & $\mathbf{0}$ & 0 & Weak Unique (H) \\
\hline Police presence influences PMI process flow & 6 & 24 & 0 & 0 & 0 & 0 & Weak Unique $(\mathrm{H})$ \\
\hline $\begin{array}{l}\text { Perceived Power Displayed by Social Service } \\
\text { Agency }\end{array}$ & $\mathbf{0}$ & $\mathbf{0}$ & $\mathbf{0}$ & $\mathbf{0}$ & 10 & 40 & Strong Unique (SS) \\
\hline Social worker requests Police assistance & 0 & 0 & 0 & 0 & 10 & 40 & Strong Unique (SS) \\
\hline
\end{tabular}

Note: $\mathrm{H}=$ Hospital; $\mathrm{P}=$ Police; $\mathrm{SS}=$ Social Service Agency.

Table 42: Perceptions of Power within the TS: TS Stakeholder Sample Pre-Change (Time 1) 
Examination of the second-order categories support the following three observations. First, the majority of respondents from each of the three TS stakeholders perceived that the Hospital displayed power within the TS before changes were implemented (i.e. high level of 'sharedness' exhibited through a strong common perception).

Second, our analysis shows that there was a low level of 'sharedness' across organizational boundaries in terms of perceptions of power displayed by the Social Service Agency and Police. Social Service Agency employees were the only respondents to mention (i.e. strong unique perception) that the Social Service Agency displayed power within the TS before the implementation of changes. Third, one in four healthcare employees were the only respondents to mention (i.e. weak unique perception) that the Police displayed power within the TS before the implementation of changes.

Examination of the first-order codes supports three additional observations. First, the data reveal two first-order codes that would be classified as common perceptions of how power is displayed in the TS before changes were implemented. More specifically, respondents from each group agreed that: (1) the Hospital displayed power by the doctor forming (or not forming) the PMI (strong common perception), and (2) the Hospital displayed power by determining when police officers can leave the Hospital (weak common perception).

Second, examination of the first level codes in Table 42 reveals that there was only one shared perception among respondents within the TS prior to the implementation of changes. More specifically, police officers (60\% of Police sample) and healthcare workers ( $72 \%$ of Hospital sample) strongly shared a similar view that the Hospital displayed power through the ability of the doctor to make decisions regarding the PMI's treatment. 
Third, our analysis identified two unique perceptions of power within the TS prior to the implementation of changes. The first involved healthcare workers uniquely perceiving another TS stakeholder's power. Prior to the implementation of changes within the TS, about a quarter of the healthcare employees in our sample (i.e. weak unique perception) commented that the presence of police officers could escalate (or de-escalate) the PMI's behavior, which would require the Hospital to act more quickly (or more slowly). This type of power was labelled 'Police presence influences PMI process flow' and can be illustrated by the following comment:

"They can actually escalate the patient [behavior]. When they're alone with the patient, they will, and it's been known to happen, they will escalate the patient [behavior] to be seen faster or to be placed faster." (Hospital Nurse)

The second unique perception provides an example of how respondents may hold unique perceptions of how their own TS stakeholder displays power within the TS. Just under half (40\%) of those in the Social Service Agency Time 1 sample perceived that their TS stakeholder displayed power when they were able to initiate Police action within the PMI process by filling out a form.

The following points summarize our analysis of how respondents thought power was displayed in the TS before the planned TS change was implemented:

- All TS stakeholders perceived that the Hospital displayed power in the TS by:

○ Deciding when police officers can leave the Hospital (strong common); and,

○ Decides whether or not the PMI is formed (weak common).

- Hospital and Police stakeholders strongly shared the view that the Hospital displayed power within the TS by having the doctor decide the PMI's treatment.

- The Social Service Agency uniquely expressed the strong view that they displayed power within the TS when social workers request Police assistance. 
- The Hospital uniquely expressed the weak view that the Police displayed power when their presence influences PMI process flow.

\subsubsection{PERCEPTIONS OF POWER WITHIN THE TS: TOTAL SAMPLE POST-CHANGE} (TIME 2)

The counts and frequencies for all perceptions of power within the TS after the implementation of the planned change are given in Table 43 for the total sample. Six first-order codes describing how power was perceived to be displayed in the TS post-change were abstracted from the data. Three of these first-order codes relate to ways that the Hospital displayed power within the TS and were grouped together within a second-order category labeled: Perceived Power Displayed by Hospital. Two of these first-order codes are associated with ways in which respondents view the Police's power within the TS and were grouped together within the secondorder category labeled: Perceived Power Displayed by Police. The final first-order code is associated with how the Social Service Agency displayed power within the TS and was classified under the second-order category: Perceived Power Displayed by Social Service Agency. The two first-order codes that were mentioned by less than $10 \%$ of the total sample will not be discussed in this section. 


\begin{tabular}{|c|c|c|}
\hline \multirow{2}{*}{$\begin{array}{c}\text { Perceptions of Power in the TS: Total } \\
\text { Sample Post-Change (Time 2) }\end{array}$} & \multicolumn{2}{|c|}{$\begin{array}{c}\text { Total } \\
\text { (n=60) }\end{array}$} \\
\cline { 2 - 4 } Perceived Power Displayed by Hospital & $\mathbf{5 3}$ & $\mathbf{8 8}$ \\
\hline Doctor decides whether PMI is formed & 37 & 62 \\
\hline Dospital staff decides when police officers can leave & 18 & 30 \\
\hline Perceived Power Displayed by Police & 17 & 28 \\
\hline Police presence influences PMI process flow & 17 & $\mathbf{2 8}$ \\
\hline Police encourage ED staff to expedite PMIs & 3 & 28 \\
\hline $\begin{array}{r}\text { Perceived Power Displayed by Social Service } \\
\text { Agency }\end{array}$ & $\mathbf{5}$ & 5 \\
\hline Social worker requests Police assistance & 5 & 8 \\
\hline
\end{tabular}

Table 43: Perceptions of Power within the TS: Total Sample Post-Change (Time 2)

Second-order Categories: During this round of interviews (i.e. post-change), respondents talked about how they perceived that the Hospital, the Police, and the Social Service Agency displayed power within the TS. Many of the respondents continued to discuss ways in which the Hospital displayed power within the PMI process ( $88 \%$ of total sample). Just over a quarter of the respondents interviewed after the implementation of the planned TS change indicated that the Police displayed power within the TS ( $28 \%$ of total sample). Less than one in ten respondents gave comments suggesting that s/he thinks the Social Service Agency displayed power within the TS ( $8 \%$ of total sample). Examination of the first-order codes give us additional information on how respondents perceived how power was displayed in the TS post-change.

Perceived Power Displayed by Hospital: The respondents we interviewed discussed the Hospital displaying power in the same three ways that were identified during analysis of prechange data: (1) by the doctor deciding whether the PMI is formed, (2) by the Hospital staff deciding when police officers can leave, and (3) by the doctor deciding the PMI's treatment. The most frequently cited way that the Hospital displays power within the TS at Time 2 was associated with the doctor deciding whether to form the PMI or not. The fact that nearly two-thirds of the 
respondents mentioned that the Hospital could display power through the forming process suggests that the doctor forming the PMI continues to be an important part of the PMI process, despite the fact that changes were introduced within the TS. The following quotes from Time 2 interviews illustrate this type of power perceived to be displayed by the Hospital:

"With the Ontario Mental Health Act being the authority, the Hospital has the right to detain someone for psychiatric assessment." (Hospital Resource Nurse)

"They have the authority to keep that person for evaluation purposes. If they deem him or her to be a threat to themselves or the public, they keep them in for evaluation purposes." (Police Constable)

The second most common kind of power perceived to be displayed by the Hospital postchange was associated with the Hospital staff determining when police officers were allowed to leave the hospital. Thirty percent of the total sample made comments that were related to the idea that the staff at the Hospital could determine when they assessed the PMI and, as a result, could determine when police officers were allowed to leave the Hospital. Example quotes from Time 2 interviews are provided below:

"I mean the police officer has to stay until the doctor has seen them and decides its okay for them to leave." (Hospital ED Nurse)

"I would probably say the Hospital does the most I mean because we're kind of at their beck and call as far as once we arrive with somebody with a mental health concern it's up to them as far as how quickly or efficiently we can get that person into the system and get our officers back out on the road right, which is my main concern." (Police Constable)

Less than a third of respondents sampled at Time 2 discussed the doctor's ability to determine the PMI's treatment when asked how power was displayed within the PMI process. 
Similar to observations made using the Time 1 data, comments made by respondents at Time 2 equated power to the doctor's ability to determine whether the PMI would be admitted to the psychiatric ward, be discharged from the Hospital, or receive different medication. The following quotes illustrate this form of perceived power as discussed during Time 2 interviews:

"The power on the MHESU end would be the ability to have the psychiatrist assess and determine whether or not the patient is staying or leaving." (Hospital Security Supervisor) "Doctors are responsible for ... what kind of treatment, how that person is going to be dealt with, whether or not there's a serious problem... they have control over the medical part of things." (Social Service Agency community support worker)

Perceived Power Displayed by Police: When asked how power was displayed in the PMI process post-change, respondents were three times more likely to discuss actions taken by the Police than behaviors displayed by Social Service Agency workers. Approximately a quarter of the employees that we interviewed felt that the Police displayed power by simply being present around the PMI. These respondents noted that the presence of a police officer could impact the PMI's behavior within the PMI process. This type of comment provided in the Time 2 interviews were given the descriptor 'Police presence influences PMI process flow'. For example:

“Well I think by their presence they display power. And sometimes that's all you need, you need to have a police officer come in a uniform and the patient will just settle down, so I think the mere presence of the police can calm a situation. It can also be calming to some patients because when they see that uniform, they go okay, I'm not going to, you know, I'm going to settle down here.... It can also aggravate a situation too." (Hospital Psychiatrist) 
"Initially to see the Police is very powerful for our clients, knowing that something is really

happening, that they need someone to contain them or to help them. And that's them [the

police officers] just being present." (Social Service Agency community support worker)

\subsubsection{PERCEPTIONS OF POWER WITHIN THE TS: TS STAKEHOLDER SAMPLE POST-CHANGE (TIME 2)}

Table 44 shows the responses given to questions associated with perceptions of power within the TS post-change broken down by TS stakeholder. Analysis of the three second-order categories are reported first, followed by the analysis of the five first-order categories.

Our analysis of the second-order categories reveals no common perceptions of TS stakeholder power after changes were implemented. Perceptions of two out of three second-order categories describing TS stakeholder power post-change were, according to our 'sharedness' criteria, shared between two TS stakeholders. Interestingly, in both cases, the two TS stakeholders strongly agreed that the third TS stakeholder displayed power. In particular, respondents from the Police (100\% of Police sample) and Social Service Agency (95\% of Social Service Agency sample) were more likely to mention ways that the Hospital displayed power after changes were implemented than Hospital employees (35\% of Hospital sample). Similarly, respondents from the Hospital (50\% of Hospital sample) and the Social Service Agency (35\% of Social Service Agency sample) were more likely to mention ways that the Police displayed power after changes were implemented than police officers ( $0 \%$ of Police sample). The final second-order category met our criteria for a weak unique perception, as only Social Service Agency employees (25\% of Social Service Agency sample) reported that they displayed power within the TS. 


\begin{tabular}{|c|c|c|c|c|c|c|c|}
\hline \multirow{2}{*}{$\begin{array}{l}\text { Perceptions of Power in the TS: TS } \\
\text { Stakeholder Sample Post-Change (Time } \\
\text { 2) }\end{array}$} & \multicolumn{2}{|c|}{$\begin{array}{c}\text { Hospital } \\
(n=20)\end{array}$} & \multicolumn{2}{|c|}{$\begin{array}{l}\text { Police } \\
(n=20)\end{array}$} & \multicolumn{2}{|c|}{$\begin{array}{l}\text { Social Service Agency } \\
(\mathrm{n}=20)\end{array}$} & \multirow{2}{*}{$\begin{array}{c}\text { Post-Change } \\
\text { Perception } \\
\text { (Common / } \\
\text { Shared / Unique) }\end{array}$} \\
\hline & \# & $\%$ & $\#$ & $\%$ & \# & $\%$ & \\
\hline Perceived Power Displayed by Hospital & 14 & 70 & 20 & 100 & 19 & 95 & Strong Shared (P, SS) \\
\hline Doctor decides whether PMI is formed & 7 & 35 & 13 & 65 & 17 & 85 & Strong Shared (P, SS) \\
\hline Hospital staff decides when police officers can leave & 8 & 40 & 8 & 40 & 2 & 10 & Weak Shared $(\mathrm{H}, \mathrm{P})$ \\
\hline Doctor decides PMI's treatment & 7 & 35 & 3 & 15 & 7 & 35 & Weak Shared (H, SS) \\
\hline Perceived Power Displayed by Police & 10 & 50 & $\mathbf{0}$ & $\mathbf{0}$ & 7 & 35 & Strong Shared (H, SS) \\
\hline Police presence influences PMI process flow & 10 & 50 & 0 & 0 & 7 & 35 & Strong Shared (H, SS) \\
\hline $\begin{array}{l}\text { Perceived Power Displayed by Social Service } \\
\text { Agency }\end{array}$ & $\mathbf{0}$ & $\mathbf{0}$ & $\mathbf{0}$ & $\mathbf{0}$ & 5 & 25 & Weak Unique (SS) \\
\hline Social worker requests Police assistance & 0 & 0 & 0 & 0 & 5 & 25 & Weak Unique (SS) \\
\hline
\end{tabular}

Note: $\mathrm{H}=$ Hospital; $\mathrm{P}=$ Police; $\mathrm{SS}=$ Social Service Agency.

Table 44: Perceptions of Power within the TS: TS Stakeholder Sample Post-Change (Time 2) 
Our analysis of the data in Table 44 shows that four out of the five first-order codes reflecting perceptions of power within the TS after changes were implemented were shared between two out of three of the TS stakeholders. More specifically:

- Police (65\% of Police sample) and Social Service Agency (85\% of Social Service Agency sample) respondents strongly shared the view that the doctor deciding whether the PMI is formed is a display of power;

- Healthcare (50\% of Hospital sample) and social service (35\% of Social Service Agency sample) workers strongly shared the view that the police displayed power, as their presence can influence the flow of the PMI process;

- Hospital (40\% of Hospital sample) and Police (40\% of Police sample) respondents weakly shared the perception that Hospital staff determining when police officers leave the Hospital was a display of the hospital's power within the TS after changes were implemented; and,

- Hospital (35\% of Hospital sample) and Social Service Agency (35\% of Social Service Agency sample) respondents weakly shared the perception that the PMI's treatment was a display of the hospital's power within the TS after changes were implemented.

In the first two of the above situations, two of the TS partners strongly shared the perception that the third TS partner exhibited power. In the second two cases, one of the TS stakeholders (i.e. Hospital) and one their partners weakly shared perceptions on how the hospital displayed power within the TS post-change. The third TS partner did not, however, share this view.

Finally, examination of the data in Table 44 reveals that there was only a single first-order code that met our criteria for a unique perception. More specifically, five of the employees in the Social Service Agency sample ( $25 \%$ of Social Service Agency sample) felt that the Social Service Agency displays power within the TS by requesting Police assistance. 
Summarizing our analysis of perceptions of power within the TS after the implementation of the planned TS change, we note the following points:

- There were no examples of common perceptions concerning how power was displayed within the TS post-change.

- Hospital and Police stakeholders shared the perception that the Hospital displayed power within the TS in the following two ways:

○ Doctors decide whether the PMI is formed (strong view); and,

- Healthcare staff decide when police officers can leave the Hospital (weak view).

- Hospital and Social Service Agency stakeholders shared the following two views:

- The Police display power within the TS when their presence influences the PMI process flow (strong view); and,

- The Hospital display power within the TS when the doctor decides the PMI's treatment (weak view).

- The Social Service Agency uniquely expressed the weak view that social workers requesting Police assistance displays power within the TS.

\subsubsection{IMPACT OF PLANNED TS CHANGE ON PERCEPTIONS OF POWER WITHIN THE TS}

This section assesses the impact of the planned TS change on perceptions of power within the TS in two complimentary ways. First, we will examine findings using cross-sectional data in Section 6.1.5.1. The findings from this stage of the analysis will give us a broad view of perceptions of power within the TS from the perspective of respondents from all three TS stakeholders. Within this section, we examine how the planned TS change may have impacted 'sharedness' of perceptions of power within the TS. It should be noted that this data set includes 
a high number of respondents in the Social Service Agency sample that withdrew from the study. This means that, while we are able to make comparisons between TS stakeholders at the group level (e.g. 'sharedness'), we were unable to conduct any analysis at the level of the individual.

In Section 6.1.5.2, we report on the second analysis, which uses panel data to investigate changes in perceptions at the individual level. The strength of the second analysis is that we are able to compare the responses that a particular individual gave at Time 1 (pre-change) to what s/he said at Time 2 (post-change). This comparison allows the researcher to detect how the planned TS change may have impacted perceptions of power within the TS at the individual level of analysis. The analysis done with the panel data is also limited, however, in that it examines responses from the 20 police officers and 20 healthcare workers who participated in Time 1 and Time 2 interviews. The fact that we are unable to do a similar analysis with the Social Service Agency sample means that we cannot make observations regarding community support workers' views of power at the individual level of analysis.

Lastly, Section 6.1.5.3 summarizes how planned TS change may have impacted perceptions of power within the TS.

\subsubsection{IMPACT OF PLANNED TS CHANGE ON PERCEPTIONS OF POWER:} ANALYSIS OF THE CROSS-SECTIONAL DATA SET

The purpose of this section is to examine how each of the TS stakeholders' views of power within the TS may have shifted over time relative to their TS partners' views. We take the following three steps to conduct this analysis.

First, as shown in Section 6.1.2, we examined Time 1 data describing how respondents in the three stakeholder groups perceived power within the TS pre-change. We classified responses (first-order codes and second-order categories) into three categories: (1) common (all three 
stakeholders perceived power displayed in this way), (2) shared (two out of three stakeholders perceived power displayed in this way), and (3) unique (only one stakeholder perceived this form of power within the TS). We also categorized these perceptions as being strong (50\% or more of respondents in each relevant stakeholder group felt power was displayed in this way) or weak (less than $50 \%$ of the respondents in each relevant stakeholder group felt power was displayed in this way).

Second, as shown in Section 6.1.4, we examined Time 2 data regarding how power was perceived to have been displayed within the TS by respondents in the three stakeholder groups six months after the planned change had been implemented. We, again, classified responses (firstorder codes and second-order categories) into three categories: (1) common, (2) shared, and (3) unique. We also categorized these perceptions as being strong or weak as noted above.

Third, we examined these data and looked for evidence of shifts in 'sharedness' (see Table 45). We operationally define a shift in 'sharedness' by comparing the classification we gave a perception of power within the TS at Time 1 (e.g. strong common) with the classification we gave the corresponding perception of power within the TS at Time 2 (e.g. weak shared). We identify three different scenarios using this analysis: (1) no shift in 'sharedness', (2) decreasing 'sharedness' (i.e. more TS stakeholders agreed on how power was displayed within the TS at Time 1 than at Time 2), and (3) increasing 'sharedness' (i.e. fewer TS stakeholders agreed on how power was displayed within the TS at Time 1 than at Time 2). In the following section, we present data supporting each of these three scenarios.

No shift in 'sharedness': Our analysis suggests that the planned TS change had little impact on how the Social Service Agency was perceived to display power within the TS. 
- $\quad$ Before and after the introduction of the planned TS change, only respondents from the Social Service Agency reported that they displayed power within the TS (strong unique at Time 1 and weak unique at Time 2) (i.e. Perceived Power Displayed by Social Service Agency).

- Only community support workers felt that they displayed power within the TS by requesting Police assistance (i.e. Social worker requests Police assistance). They held these views at Time 1 and at Time 2 (strong unique at Time 1 and weak unique at Time 2). The above data support the idea that, even though the planned TS change did not impact the extent that the Hospital or the Police observed power within the Social Service Agency, it seems to have lessened the extent that community support workers feel they display power within the TS.

Decreasing 'sharedness': Although all three TS stakeholders mentioned that the Hospital displayed power within the TS before changes were introduced, only two of them felt the Hospital displayed power within the TS after the planned TS change.

- All three TS stakeholders indicated that the Hospital displayed power within the TS before any change occurred (strong common) (i.e. Perceived Power Displayed by Hospital). After the introduction of the planned TS change, however, only the Police and Social Service Agency felt that the Hospital displayed power within the TS (strong shared between Police and Social Service Agency).

- In Time 1 interviews, the Hospital and Police indicated that they felt the doctor deciding the PMI's treatment was a form of Hospital power (strong shared between Hospital and Police) (i.e. Doctor decides PMI's treatment). The respondents in the Police did not, however, observe that this was a form of Hospital power post-change (weak shared between Hospital and Social Service Agency). 
- In Time 1 interviews, all three TS stakeholders indicated that the Hospital staff deciding when police officers can leave the Hospital was a form of power (strong common) (i.e. Hospital staff decides when police officers can leave). At Time 2, however, community support workers did not feel this was a form of power within the TS (weak shared between Hospital and Police).

- At Time 1, all three TS stakeholders indicated that the doctor deciding whether the PMI is formed was a type of Hospital power (weak common) (i.e. Doctor decides whether PMI is formed). At Time 2, even though the Police and Social Service Agency still thought this was a display of Hospital power, healthcare workers did not (strong shared between Police and Social Service Agency).

In this case, it would appear that the planned TS change substantially affected how respondents view how the Hospital displays power within the TS. More specifically, healthcare workers do not feel that they display power within the TS as much after the planned TS change as they did before. Respondents from their TS partners think that how the Hospital displays power within the TS has changed.

Increasing 'sharedness': Only one stakeholder mentioned that the Police displayed power within the TS before changes were introduced, however, two of them felt the Police displayed power within the TS after the planned TS change.

- Before the introduction of the planned TS change, only the Hospital mentioned that they felt the Police displayed power within the TS (weak unique) (i.e. Perceived Power Displayed by Police). Post-change, the Hospital and the Social Service Agency felt that the Police displayed power within the TS (strong shared between Hospital and Social Service Agency).

- In Time 1 data, the Hospital mentioned that Police presence influenced the PMI process flow (unique) (i.e. Police presence influences PMI process flow). At Time 2, the Hospital and Social 
Service Agency indicated that the presence of the Police was a form of Police power (strong shared between Hospital and Social Service Agency).

These findings suggest that, while only the Hospital thought the Police had power at Time 1, the Hospital and Social Service Agency felt the Police held power within the TS at Time 2.

We summarize these findings to the following three points:

- The data suggests that, even though the planned TS change did not impact the extent that the Hospital or the Police observed power within the Social Service Agency, it seems to have lessened the extent that community support workers feel they display power within the TS.

- The planned TS change seems to have decreased the extent to which those in the Hospital sample perceive that the Hospital displayed power within the TS.

- The planned TS change seems to have increased the extent to which those in the Social Service Agency sample perceive that the Police displayed power within the TS.

To get a better understanding why the 'sharedness' may have shifted in this manner, we turn to our analysis at the individual level in the following section. 


\begin{tabular}{|c|c|c|c|}
\hline Perceptions of Power in the TS Over Time & $\begin{array}{c}\text { Pre-Change } \\
\text { Perception (Common } \\
\text { / Shared / Unique) }\end{array}$ & $\begin{array}{c}\text { Post -Change } \\
\text { Perception } \\
\text { (Common / Shared } \\
\text { / Unique) }\end{array}$ & $\begin{array}{c}\text { Shift in } \\
\text { 'Sharedness' }\end{array}$ \\
\hline Perceived Power Displayed by Hospital & Strong Common & Strong Shared (P, SS) & Decrease \\
\hline Doctor decides PMI's treatment & Strong Shared (H, P) & Weak Shared (H, SS) & No Shift \\
\hline Hospital staff decides when police officers can leave & Strong Common & Weak Shared (H, P) & Decrease \\
\hline Doctor decides whether PMI is formed & Weak Common & Strong Shared (P, SS) & Decrease \\
\hline Perceived Power Displayed by Police & Weak Unique (H) & Strong Shared (H, SS) & Increase \\
\hline Police presence influences PMI process flow & Weak Unique (H) & Strong Shared (H, SS) & Increase \\
\hline Perceived Power Displayed by Social Service Agency & Strong Unique (SS) & Weak Unique (SS) \\
\hline Social worker requests Police assistance & Strong Unique (SS) & Weak Unique (SS) \\
\hline
\end{tabular}

Note. Only responses associated with first-order codes that were given a common, shared, or unique label at Time 1 and/or at Time 2 are shown.

Table 45: Perceptions of Power within the TS Over Time: Cross-Sectional Data 


\subsubsection{IMPACT OF PLANNED TS CHANGE ON PERCEPTIONS OF POWER:}

ANALYSIS OF THE PANEL DATA SET

Table 46 summarizes changes to perceptions of power within the TS over time by using panel data to compare what a respondent said at Time 1 to what $\mathrm{s} /$ he mentioned at Time 2 . When analyzing the panel data, we consider a perception of power changing when a substantive number of respondents gave a response either: (1) during the Time 1 interview but not during the Time 2 interview, or (2) during the Time 2 interview but not during the Time 1 interview. We arbitrarily define a substantive difference between Time 1 and Time 2 responses as a difference of 5 respondents per TS stakeholder group (i.e. $25 \%$ of a TS stakeholder sample).

It should be noted that only those perceptions of power that met our criteria for a substantive difference between Time 1 and Time 2 are shown in Table 46. A full analysis of all perceptions of power using the panel data set is found in the Appendix.

Once we identified a change in perception of power over time, we conducted a follow-up analysis by examining the comments made by individuals who we classified as having a changed view. This follow-up analysis specifically looked for patterns emerging from the data that could explain how the planned TS change may have impacted views of power over time. We first present changes to perceptions of power associated with the Hospital, followed by that of the Police. 


\begin{tabular}{|c|c|c|c|}
\hline & $\begin{array}{l}\text { Perception } \\
\text { mentioned }\end{array}$ & $\begin{array}{c}\text { Hospital } \\
(\mathbf{n}=\mathbf{2 0})\end{array}$ & $\begin{array}{l}\text { Police } \\
(\mathrm{n}=\mathbf{2 0})\end{array}$ \\
\hline \multicolumn{4}{|l|}{$\begin{array}{l}\text { Perceived Power Displayed by } \\
\text { Hospital }\end{array}$} \\
\hline \multirow{2}{*}{$\begin{array}{r}\text { Hospital staff decides when police } \\
\text { officers can leave }\end{array}$} & $\begin{array}{l}\text { Said T1 } \\
\text { not T2 }\end{array}$ & $\begin{array}{c}6 \\
(30 \%) \\
\end{array}$ & $\begin{array}{c}9 \\
(45 \%) \\
\end{array}$ \\
\hline & $\begin{array}{l}\text { Said T2 } \\
\text { not T1 }\end{array}$ & $\begin{array}{c}4 \\
(20 \%)\end{array}$ & $\begin{array}{c}4 \\
(20 \%)\end{array}$ \\
\hline \multirow{2}{*}{ Doctor decides PMI's treatment } & $\begin{array}{l}\text { Said T1 } \\
\text { not T2 }\end{array}$ & $\begin{array}{c}15 \\
(75 \%)\end{array}$ & $\begin{array}{c}15 \\
(75 \%)\end{array}$ \\
\hline & $\begin{array}{l}\text { Said T2 } \\
\text { not T1 }\end{array}$ & $\begin{array}{c}4 \\
(20 \%)\end{array}$ & $\begin{array}{c}3 \\
(15 \%) \\
\end{array}$ \\
\hline \multirow{2}{*}{$\begin{array}{r}\text { Doctor decides whether PMI is } \\
\text { formed }\end{array}$} & $\begin{array}{l}\text { Said T1 } \\
\text { not T2 }\end{array}$ & $\begin{array}{c}3 \\
(15 \%)\end{array}$ & $\begin{array}{c}1 \\
(5 \%)\end{array}$ \\
\hline & $\begin{array}{l}\text { Said T2 } \\
\text { not T1 }\end{array}$ & $\begin{array}{c}4 \\
(20 \%)\end{array}$ & $\begin{array}{c}10 \\
(50 \%)\end{array}$ \\
\hline \multicolumn{4}{|l|}{$\begin{array}{l}\text { Perceived Power Displayed by } \\
\text { Police }\end{array}$} \\
\hline \multirow{2}{*}{$\begin{array}{r}\text { Police presence influences PMI } \\
\text { process flow }\end{array}$} & $\begin{array}{l}\text { Said T1 } \\
\text { not T2 }\end{array}$ & $\begin{array}{c}1 \\
(5 \%)\end{array}$ & $\begin{array}{c}0 \\
(0 \%)\end{array}$ \\
\hline & $\begin{array}{l}\text { Said T2 } \\
\text { not T1 }\end{array}$ & $\begin{array}{c}5 \\
(25 \%)\end{array}$ & $\begin{array}{c}0 \\
(0 \%)\end{array}$ \\
\hline
\end{tabular}

Note. $\mathrm{T} 1=$ Time $1 . \mathrm{T} 2=$ Time 2 . Shaded cells identify substantive changes in perceptions over time.

Table 46: Perceptions of Power within the TS Over Time: Representative Panel Data

Examination of Table 46 supports the following observations. Our analysis of these findings suggest that the planned TS change may have decreased the Hospital's power within the TS over time. More specifically, while $30 \%$ of the Hospital and $45 \%$ of the Police samples identified that Hospital staff deciding when police officers can leave the Hospital as an example of how the Hospital displayed power within the TS before the planned TS change, none of these individuals provided this response post-change. To get a better understanding why the views of these 6 healthcare workers and 9 police officers changed over time, we examined their responses in more detail. This follow-up analysis showed that all fifteen of these respondents felt that one of the changes introduced into the TS (i.e. the intervention and escalation policy) added more 
pressure on the Hospital to assess PMIs quicker. This increased pressure seems to have reduced the Hospital's power to restrict the flow of PMIs entering the ED.

“Increased pressure on doctors to get to these types of patients." (Hospital ED Nurse)

"There is much more pressure on the intake of the emergency room. We are not able to restrict the flow of patients coming. In a sense, when we are ready for the patient, we have to take them. So we've lost a bit of power over patients entering emerg... and we haven't really gained power on getting the patient to psychiatry [downstream in the process]" (Hospital ED Physician)

"I think it's maybe taken a little power from the Hospital because now there's that expectation that, barring anything unforeseen, the apprehended party is supposed to be processed within a couple of hours. Ultimately, the power still remains with the Hospital, but I think maybe a little more to the Police because there is that expectation." (Police Constable)

Our analysis suggests that the planned TS change also decreased the Hospital's power in a second way. Three quarters of the individuals in the Hospital and Police samples stated prior to the planned TS change that the doctor deciding the PMI's treatment demonstrated power for the Hospital. None of these individuals gave this response at Time 2. Our follow-up investigation of the responses given by these respondents suggested that the increased pressure on the ED staff to assess and treat the PMI (i.e. result from the intervention and escalation policy) may have also impacted who is involved in treating the PMI. For example, one of the tactics that the Hospital used to expedite service for PMIs was engaging crisis workers earlier within the PMI process (see Increased Engagement of MHESU staff in Section 5.1.2). Our analysis of the responses shows that the increased engagement of crisis workers has resulted in crisis workers acting as a filter in 
the PMI process before the doctor's involvement. One police constable commented at Time 2: " $A$ lot of the time we see crisis workers before the doctor." This gives crisis workers the opportunity to learn much more about the PMI, as suggested by an ED resource nurse: "A crisis [worker] spends about 40 minutes to an hour with them to dive a little bit deeper into what brought them here." This initial assessment also allows the crisis worker to determine who else needs to be involved in their care (e.g. ED doctor, psychiatrist). One healthcare worker described the role of crisis workers by saying “crisis [workers] assess they're [the PMI are], sort of, certifiable and have a mental illness issue." It would appear from this analysis that the introduction of crisis workers earlier into the PMI process may have reduced the amount of power that doctors have over treating the PMI.

It should be noted that, while our coding scheme might reflect the Hospital losing power as a result of doctors losing power to treat PMIs, the above follow-up analysis suggests that the power shifted from doctors to crisis workers. Interestingly, however, no respondent directly commented that crisis workers had more power within the PMI process after the implementation of the planned TS change.

Our analysis of the data found in Table 46 also provides some evidence that the planned TS change increased the Hospital's power over time, at least in the eyes of police officers. More specifically, half of the police respondents indicated that the Hospital can display power within the TS by deciding whether to form the PMI post-change. None of these officers noted this form of power in the pre-change interviews. Our follow-up analysis of the comments from the 10 police officers who gave this response provided two different explanations why this might have occurred. First, as noted in Chapter 5, the planned TS change increased communication between the Police and the Hospital. It appears that, with this increased interorganizational communication, some 
police officers had the expectation that the doctor would take into consideration what the Police were saying when deciding whether to form the PMI or not. There were several examples provided by these 10 officers that spoke to the idea that, despite police officers conversing with the doctor, the doctor continued to form PMIs the way they wanted. This may have resulted in the Police perceiving that the doctor displayed more power within the TS prior to the planned TS change. "Sometimes you'll go to a doctor and you'll explain to the doctor what's going on. The doctor will form the person without even talking to them. And then other times the doctor will have a conversation with them after they've talked to you, and they're, like, no, they're not forming them, they just need to go home and take a rest or whatever. If they aren't going to listen to us, why are we even talking?" (Police Constable)

Second, there was also evidence from these 10 officers that there was an increased reliance on doctors to appropriately assess whether the individual in fact had mental health issues (i.e. should have been apprehended). In their comments below, one police officer suggests that Police are apprehending more PMIs because there are shorter wait times, while another suggests that Police are depending on the expertise of the doctors to make the appropriate assessment.

"Knowing human nature and the way coppers are, it's definitely a factor... I could see it being a factor if let's say you're three hours or two hours from the end of your shift, or your lunch, or whatever, and you're like, holy crap the last time I was there I sat there for eight hours, or oh yes, I was only there an hour and a half last time I was there, so let's hook him up and take him in and see what the doctor says." (Police Constable)

"I think we defer to the experts [doctors] more often than we should... but our process is not to judge one way or the other on any circumstance whether it be criminal or mental health. Ours is to assess the best we can and deliver to the professionals that can make the 
decision... It's not us to decide whether somebody is or is not mentally stable or safe." (Police Constable)

In this second scenario, the increased reliance on doctors to assess PMIs appears to have empowered doctors, and thus increased the perceived power of the Hospital within the TS.

Lastly, findings show that there was one change to how respondents perceived the Police displaying power within the TS. Our analysis of these findings suggest that the planned TS change may have given the Police power within the TS over time. In particular, five individuals in the Hospital sample ( $25 \%$ of Hospital sample) noted that, post-change, the Police may display power within the TS by influencing the flow within the PMI process. None of these individuals indicated the Police displaying this form of power before the planned TS change. Follow-up analysis suggests that the intervention and escalation policy has made Police more vocal within the PMI process, and has impacted healthcare workers' views of how the Police display power within the TS.

"It's empowered the police a little bit. They are a little bit more to be more vocal on expediting themselves through the department." (ED Resource Nurse)

"I can say it slightly changed who has power because the police are now demanding because this is the process that we have to follow, they have more power in terms of handing the person over." (ED Nurse)

We summarize our analysis of panel data with the following four points:

- The implementation of the intervention and escalation policy appears to have decreased the Hospital's power to decide when police officers can leave the Hospital. 
- The implementation of the intervention and escalation policy may have shifted some of the power within the Hospital, such that doctors are perceived to have less influence over treating PMIs and crisis workers are perceived to have more influence over treating PMIs.

- The planned TS change appears to have increased the doctor's power to form PMIs in the eyes of police officers.

- The intervention and escalation policy may have increased Police power within the TS by empowering them to be more vocal in expressing their demands.

\subsubsection{SUMMARY OF HOW THE PLANNED TS CHANGE MAY HAVE IMPACTED PERCEPTIONS OF POWER WITHIN THE TS}

Throughout Section 6.1.5, we explored changes to perceptions of power within the TS in order to get a better understanding of how the planned TS change may have impacted TS stakeholder power. The findings from our two analyses, one performed using cross-sectional data and the other performed using panel data, support the idea that the planned TS change has shifted power within the TS from the Hospital to the Police.

More specifically, our analyses suggest that the planned TS change decreased Hospital power, particularly in the eyes of healthcare workers. The implementation of the intervention and escalation policy appears to have decreased the Hospital's power to decide when police officers can leave the Hospital, and dispersed power within the Hospital such that doctors have less influence and crisis workers have more influence over the PMI's treatment.

Our analysis also suggests that the planned TS change increased the power of the Police to influence the PMI process and that, as a result, more stakeholders became aware of how the Police displayed power within the TS. This increased power may be related to the implementation of the 
intervention and escalation policy, as this policy gave police officers more opportunities to communicate with healthcare providers and vocalize their demands.

\subsection{PERCEPTIONS OF LEGITIMACY WITHIN THE TS}

The presentation of findings associated with perceptions of TS stakeholder legitimacy is broken down as follows. We present the results of the perceptions of legitimacy within the TS based on the cross-sectional data set in the first four sections. In particular, we present Time 1 findings first for the total sample (Section 6.2.1), and then examine the findings at the TS stakeholder level (Section 6.2.2). This is followed by a summary of our findings with respect to perceptions of legitimacy within the TS after changes were implemented. The post-change results for the total sample are presented first (Section 6.2.3), followed by our analysis of data by TS stakeholder (Section 6.2.4). The final section, Section 6.2.5, explores how the planned TS change may have impacted TS stakeholder legitimacy by examining both cross-sectional and panel data showing changes to perceptions of legitimacy within the TS over time.

\subsubsection{PERCEPTIONS OF LEGITIMACY WITHIN THE TS: TOTAL SAMPLE PRE- CHANGE (TIME 1)}

Data analysis of the Time 1 interviews identified eleven ways that respondents talked about legitimacy within the TS prior to the introduction of the planned TS change (i.e. first-order codes). Four of these first-order codes related to ways in which respondents view the Police's legitimacy within the TS and were grouped together within a second-order category entitled: Perceptions of Legitimate Police Actions. Four of these first-order codes were associated with ways in which respondents view the Hospital's legitimacy within the TS and were grouped together within a second-order category labeled: Perceptions of Legitimate Hospital Actions. The three remaining first-order codes related to respondents' views of Social Service Agency legitimacy within the TS 
and formed the second-order category: Perceptions of Legitimate Social Service Agency Actions. All first-order codes are grouped with their respective second-order category and displayed in Table 47, in descending order by respondent count. Although they are included in Table 47, two Police actions, two Hospital actions, and one Social Service Agency action were identified by respondents as typifying how these stakeholders behaved legitimately within the TS were mentioned by less than $10 \%$ of the total sample and will not be discussed in this section.

\begin{tabular}{|c|c|c|}
\hline \multirow{2}{*}{$\begin{array}{c}\text { Perceptions of Legitimacy within the TS: } \\
\text { Total Sample Pre-change (Time 1) }\end{array}$} & \multicolumn{2}{|c|}{$\begin{array}{c}\text { Total } \\
(n=75)\end{array}$} \\
\hline & $\#$ & $\%$ \\
\hline Perceptions of Legitimate Hospital Actions & 46 & 61 \\
\hline Healthcare workers assess PMIs & 36 & 48 \\
\hline Healthcare workers treat PMIs & 30 & 40 \\
\hline Hospital can discipline PMI & 6 & 8 \\
\hline $\begin{array}{r}\text { Other Hospital legitimacy (i.e. healthcare policy, } \\
\text { people seek out hospitals) }\end{array}$ & 2 & 3 \\
\hline Perceptions of Legitimate Police Actions & 71 & 95 \\
\hline Police apprehend PMI under Mental Health Act & 57 & 76 \\
\hline Police keep community safe & 19 & 25 \\
\hline $\begin{array}{r}\text { Police provide background information about PMI to } \\
\text { doctors }\end{array}$ & 5 & 7 \\
\hline Police can maintain order in Hospital & 1 & 1 \\
\hline $\begin{array}{l}\text { Perceptions of Legitimate Social Service Agency } \\
\text { Actions }\end{array}$ & 23 & 31 \\
\hline Social Service Agency workers initiate PMI process & 14 & 19 \\
\hline $\begin{array}{r}\text { Social Service Agency worker supports PMI in } \\
\text { community }\end{array}$ & 14 & 19 \\
\hline $\begin{array}{r}\text { Other Social Service Agency legitimacy during PMI } \\
\text { process }\end{array}$ & 5 & 7 \\
\hline
\end{tabular}

Table 47: Perceptions of Legitimacy within the TS: Total Sample Pre-Change (Time 1)

Second-Order Categories: When we asked employees about legitimacy within the PMI process, almost everyone ( $95 \%$ of total sample) identified actions taken by the Police. Two thirds of the respondents talked about things the Hospital did within the TS (61\% of total sample). A third of the respondents provided at least one legitimate Social Service Agency action (31\% of total sample). 
Perceptions of Legitimate Hospital Actions: According to respondents interviewed prior to the implementation of changes (i.e. Time 1), two activities undertaken by the Hospital contributed to the perception that it was a legitimate stakeholder within the PMI process: (1) healthcare workers assess the PMI and (2) healthcare workers treat the PMI. Details on the types of comments grouped within each of these first-order codes are given below.

First, nearly half of the respondents interviewed prior to the implementation of changes responded to our questions on legitimacy by talking about how Hospital staff assess PMIs when they are brought to the hospital by the police. In particular, many of these respondents noted the importance of ED physicians in the PMI process, and how their assessment of the PMI was important to the flow of the PMI process. Police officers tended to describe the ED physician assessment in terms of the ED physician 'forming' the PMI. These types of comments were grouped and given the label 'Healthcare workers assess PMIs'. The following quotes illustrate how respondents describe this form of Hospital legitimacy:

"We have training, expertise, and a mandate to provide a comprehensive psychiatric assessment of the patient." (Hospital MHESU Psychiatrist)

"They're the ones that have the resources available for us to turn that individual over for assessment... Once the doctor forms the [PMI], we can leave." (Police Sergeant)

"The Hospital plays their part because they have a team of psychiatrists and nurses who have a better understanding of mental health issues... they have the expertise under one roof to assess these people." (Social Service Agency Frontline ACT)

A substantial number of respondents ( $40 \%$ of total sample) felt that Hospital staff demonstrated their legitimacy within the PMI process by treating individuals with mental illness. While the majority of these respondents just mentioned that healthcare workers provided treatment 
to PMI, others noted specific forms of treatment (e.g., injections, drugs, medication plans). We grouped these two types of comments together within one first-order code which we labeled 'Healthcare workers treat PMIs'. Illustrative quotes describing this first-order code are provided by employees in each of the three TS stakeholders below:

"It's a legislated role for the physician and for the psychiatrist. They look after treating patients. I suppose this legislation stretches to cover everyone in the Hospital for that matter.” (Hospital Administrator)

"Doctors give out meds to these guys ... to prevent the whole... Dr. Jekyll and Mr. Hyde routine." (Police Constable)

"I would love to be able to say the hospitals are [legitimate]. I think that if there were more psychiatric facilities, then the general level-one hospitals wouldn't necessarily be as required. They [PMIs] could go directly to a psychiatric facility. But I think in the system that we have right now, the Hospital is definitely legitimate in that they are the provider of the treatment." (Social Service Agency Frontline Justice)

Perceptions of Legitimate Police Actions: According to the employees we interviewed, the Police are legitimate stakeholders within the PMI process because they perform the following three activities: (1) they apprehend PMIs under the mental health act, (2) they keep the community safe, and (3) they can criminalize individuals. Details on each of these activities are provided below.

The most frequently mentioned activity undertaken by police which demonstrated their legitimacy within the PMI process was given the descriptor 'Police apprehend PMIs under the mental health act'. This type of action was mentioned by 57 out of 75 ( $76 \%$ of total sample) respondents in response to our query on how police demonstrated legitimacy within the TS. The following quotes, which were provided by employees in each of the three TS stakeholder groups 
prior to the implementation of changes, illustrate police officers apprehending PMIs under the Mental Health Act:

"The Police have the authority to apprehend someone or to detain them against their will for their own wellbeing... Anything external to the Hospital, the Police have full jurisdiction.” (Hospital ED Physician)

"The officers will apprehend them under the Metal Health Act, the party is handcuffed, searched as per our directives... to look for any object that may endanger him or us and then they'll be transported to the nearest emergency ward with a mental health facility attached to it." (Police Sergeant)

“The Police, after asking a set of questions in determining that she [a PMI] wasn't well and she needed to be hospitalised, basically brought her in the cruiser to the Hospital." (Social Service Agency Frontline ACT)

Comments included in the second most frequently mentioned way in which the police demonstrated legitimacy within the TS prior to the change spoke to activities that involved police officers keeping the community safe. This type of comment, mentioned by a quarter of the total sample, was grouped together and given the first-order code 'Police keep community safe'. The following remarks illustrate the types of activities included within this first-order code:

"The Police, by virtue of legislation, are provided with the legitimacy to ensure safety of the individual and the public. They have the legitimacy to act. They have a requirement to act." (Hospital Psychiatrist)

"I think the Police inevitably are going to be involved with these individuals, because at times they can act out or be violent or create that call for service." (Police Constable) 
"In a perfect world, Police wouldn't need to be a legitimate stakeholder, but unfortunately, because of the nature of mental illness at times they are a legitimate stakeholder in that they have to keep our community safe." (Social Service Agency Frontline Justice)

Perceptions of Legitimate Social Service Agency Actions: When asked during the Time 1 interviews why the Social Service Agency could be considered a legitimate stakeholder within the PMI process, respondents talked about two activities that were performed by this agency: (1) Social Service Agency workers initiate PMI process, and (2) Social Service Agency worker supports PMIs in the community. In the first case, one in five respondents equated legitimacy with the idea that the Social Service Agency could initiate the PMI process. More specifically, respondents mentioned that the fact that social workers would call 911, or present their case to the Justice of the Peace, when they thought that a PMI was about to have a mental health crisis made them a legitimate player within the PMI process. The descriptor 'Social Service Agency workers initiate the PMI process' was given to these types of comments. For example:

"After you perform an assessment and you've determined that the client (a) needs help and (b) is unwilling to get help or go [to the Hospital] voluntarily, then there's a process whereby you'd either go to a Justice of the Peace or you would go to the police station and say, I have significant concerns that my client is at risk of hurting themselves for example." (Social Service Agency Frontline Justice)

The second action that was mentioned during our discussions of whether or not the Social Service Agency was a legitimate stakeholder within the PMI process (mentioned by one in five respondents) spoke to the fact that social workers provided long term care, case management, medication, food, conversation, direction, and guidance for PMIs in the community. We grouped these comments together within a first-order code we labeled 'Social Service Agency worker 
supports PMIs in the community'. Illustrative quotes for Social Service Agency workers supporting PMIs in the community are provided below:

"I would say care can be provided in ways without the Hospital necessarily being involved.... I know there are psychiatrists in the community." (Hospital Psychiatrist)

"As a professional working with clients with mental illness, I guess as a worker if you see something, your client lacking in some areas, it is your duty to really inform them [the PMI] of your concerns... and they really listen to us... but sometimes the client doesn't see where you're coming from so it's difficult to work toward a goal." (Social Service Agency Frontline ACT)

\subsubsection{PERCEPTIONS OF LEGITIMACY WITHIN THE TS: TS STAKEHOLDER SAMPLE PRE-CHANGE (TIME 1)}

Table 48 is similar to Table 47, but in this case the data are broken down by TS stakeholder to allow us to determine similarities and differences in perceptions of legitimacy across TS stakeholders. Analysis of the second-order categories are reported first, followed by the analysis of first-order codes.

Our analysis of the three second-order categories related to perceptions of legitimacy within the TS at Time 1 identified that one was strongly held as a common perception, one was strongly shared between two out of three of the TS stakeholders (Police and Hospital), and one was strongly held by only one TS stakeholder (Social Service Agency). More specifically, nearly all respondents felt that Police's actions gave them legitimacy within the TS; two-thirds of the individuals included in the Police and the Hospital samples perceived that the Hospital's actions within the PMI process gave them legitimacy (as did a smaller but still substantive percent of those 
in the Social Service Agency sample); and three quarters of community support workers felt that the action(s) taken by the Social Service Agency made them legitimate stakeholders within the TS. Examination of the first-order codes included within each second-order category shed light on the above findings. Our analysis identified three common perceptions prior to the introduction of the planned TS change. We classified one as a strong common perception, as the majority of respondents from each of the three TS stakeholder groups perceived that the action of apprehending PMIs under the mental health act was legitimate. We classified the other two as weak common perceptions: (1) approximately a quarter of respondents from each TS stakeholders' sample mentioned that the Police demonstrated legitimacy within the PMI process when they took actions to keep the community safe, and (2) approximately one in three of the respondents in each TS stakeholders' sample mentioned that healthcare workers demonstrated legitimacy within the PMI Process when they provided the treatment to PMIs. 


\begin{tabular}{|c|c|c|c|c|c|c|c|}
\hline \multirow{2}{*}{$\begin{array}{c}\text { Perceptions of Legitimacy within the TS: } \\
\text { TS Stakeholder Sample Pre-Change } \\
\text { (Time 1) }\end{array}$} & \multicolumn{2}{|c|}{$\begin{array}{l}\text { Hospital } \\
(\mathrm{n}=25)\end{array}$} & \multicolumn{2}{|c|}{$\begin{array}{l}\text { Police } \\
(n=25)\end{array}$} & \multicolumn{2}{|c|}{$\begin{array}{l}\text { Social Service } \\
\text { Agency } \\
(\mathbf{n}=\mathbf{2 5})\end{array}$} & \multirow{2}{*}{$\begin{array}{l}\text { Pre-Change } \\
\text { Perception (Common } \\
\text { / Shared / Unique) }\end{array}$} \\
\hline & \# & $\%$ & \# & $\%$ & $\#$ & $\%$ & \\
\hline Perceptions of Legitimate Hospital Actions & 17 & 68 & 17 & 68 & 12 & 48 & Strong Shared (H, P) \\
\hline Healthcare workers assess PMIs & 13 & 52 & 15 & 60 & 8 & 32 & Strong Shared $(\mathrm{H}, \mathrm{P})$ \\
\hline Healthcare workers treat PMIs & 10 & 40 & 9 & 36 & 11 & 44 & Weak Common \\
\hline Perceptions of Legitimate Police Actions & 24 & 96 & 25 & 100 & 22 & 88 & Strong Common \\
\hline Police apprehend PMIs under Mental Health Act & 16 & 64 & 25 & 100 & 16 & 64 & Strong Common \\
\hline Police keep community safe & 7 & 28 & 8 & 32 & 4 & 16 & Weak Common \\
\hline Police provide information to doctor & 0 & 0 & 5 & 20 & 0 & 0 & Weak Unique (P) \\
\hline $\begin{array}{l}\text { Perceptions of Legitimate Social Service Agency } \\
\text { Actions }\end{array}$ & 5 & 20 & $\mathbf{0}$ & $\mathbf{0}$ & 18 & 72 & Strong Unique (SS) \\
\hline Social Service Agency workers initiate PMI process & 0 & 0 & 0 & 0 & 14 & 56 & Strong Unique (SS) \\
\hline $\begin{array}{r}\text { Social Service Agency worker supports PMI in } \\
\text { community }\end{array}$ & 4 & 16 & 0 & 0 & 10 & 40 & Weak Unique (SS) \\
\hline
\end{tabular}

Note: $\mathrm{H}=$ Hospital; $\mathrm{P}=$ Police; $\mathrm{SS}=$ Social Service Agency.

Table 48: Perceptions of Legitimacy within the TS: TS Stakeholder Sample Pre-Change (Time 1) 
It is also interesting to note that, before the implementation of the planned TS change, respondents in the healthcare (52\% of Hospital sample) and Police (60\% of Police sample) samples strongly shared the view that the fact that healthcare workers assess the PMI when they arrived in the emergency department gave them legitimacy within the PMI process. The fact that Social Service Agency workers were less likely to express this view might be due to the fact that social workers are infrequently present when doctors assess PMIs brought to the Hospital by police officers. As such, they have fewer opportunities to see the 'value proposition' of such an action (Drori \& Honig, 2013).

According to our 'sharedness' criteria, Police respondents held a weak unique perception about themselves. More specifically, analysis of the data show that Police respondents weakly held the unique perception that providing information about the PMI to doctors was a legitimate activity.

Finally, our analysis of the data also shows that respondents from the Social Service Agency held strong and weak unique perceptions that they behaved in a manner that typified legitimacy within the TS. Social Service Agency respondents strongly held the unique perception that initiating the PMI process (e.g. calling 911) was legitimate within the TS, and weakly held the unique perception that supporting the PMI in the community gave them legitimacy within the TS.

The following points summarize our analysis of how respondents perceived legitimacy within the TS before the implementation of the planned TS change:

- All three TS stakeholders agreed on the following three perceptions:

- Police apprehending PMIs under the mental health act demonstrated legitimacy within the TS;

- Police keeping the community safe showed legitimacy within the TS; and, 
- Healthcare workers treating PMIs was legitimate within the TS.

- Hospital and Police stakeholders strongly shared the view that healthcare workers assessing PMIs demonstrated legitimacy within the TS.

- The Social Service Agency perceived that they demonstrated legitimacy when they:

- Initiated the PMI process; and,

- Supported PMIs in the community.

- The Police expressed the view that they demonstrated legitimacy when they provided information to doctors.

\subsubsection{PERCEPTIONS OF LEGITIMACY WITHIN THE TS: TOTAL SAMPLE POST- CHANGE (TIME 2)}

The frequencies for all first-order codes relating to actions that were perceived to represent legitimacy within the TS post implementation of the planned changes are given in Table 49 for the total sample. Eight first-order codes related to legitimacy in the TS at Time 2 were abstracted from the data. Two of these first-order codes were associated with ways in which the respondents view the Hospital's legitimacy within the TS and were grouped together within a second-order category labeled: Perceptions of Legitimate Hospital Actions. Three of these first-order codes were linked to ways in which the respondents view the Police's legitimacy within the TS and formed the second-order category: Perceptions of Legitimate Police Actions. The final three firstorder codes describe ways in which respondents view the Social Service Agency's legitimacy within the TS and were grouped together within the second-order category: Perceptions of Legitimate Social Service Agency Actions. One of these perceptions was mentioned by less than $10 \%$ of the sample and will not be discussed in this section (see Table 49). Details on behaviours mentioned by at least $10 \%$ of the sample are provided in the section below. 


\begin{tabular}{|r|c|c|}
\hline \multirow{2}{*}{$\begin{array}{c}\text { Perceptions of Legitimacy within the TS: } \\
\text { Total Sample Post-Change (Time 2) }\end{array}$} & \multicolumn{2}{|c|}{$\begin{array}{c}\text { Total } \\
\text { (n=60) }\end{array}$} \\
\cline { 2 - 4 } Perceptions of Legitimate Hospital Actions & $\mathbf{3 4}$ & $\mathbf{5 7}$ \\
\hline Healthcare workers assess PMIs & 34 & 57 \\
\hline Healthcare workers treat PMIs & 13 & 22 \\
\hline Perceptions of Legitimate Police Actions & $\mathbf{4 9}$ & $\mathbf{8 2}$ \\
\hline Police apprehend PMIs under mental health act & 49 & 82 \\
\hline $\begin{array}{r}\text { Police keep community safe } \\
\text { doctor }\end{array}$ & 17 & 28 \\
\hline Perceptions of Legitimate Social Service Agency \\
Actions
\end{tabular}

Table 49: Perceptions of Legitimacy within the TS: Total Sample Post-Change (Time 2)

Second-order categories: When we asked respondents our line of questioning about legitimacy after changes were implemented within the TS, the majority of respondents provided examples of how the Police demonstrated legitimacy within the TS ( $82 \%$ of total sample). Over half of the respondents indicated that the Hospital displays legitimacy within the TS (57\% of total sample). About a third of the respondents mentioned that the Social Service Agency (35\% of total sample) demonstrated legitimacy within the TS.

Perceptions of Legitimate Hospital Actions: Over half of the respondents (57\% of the total sample) interviewed after changes were implemented identified the assessment of the PMI during the PMI process by ED physicians, ED nurses, and psychiatrists as an indicator of the Hospital's legitimacy within the PMI process. The descriptor 'Healthcare workers assess PMIs' was given to comments such as the ones below: 
"I use a combination of things. If the patient is able to define what their issues are... and a set of tools that help us to define what they have, such as using that DSM criteria... they help give us a diagnosis." (Hospital Psychiatrist)

"As far as legitimacy, it's not on us to decide who is mentally stable or not. That's what the professional [doctor] does when they assess the person, that's why they get the big degrees. They're the ones who can look at someone and say whether they're mental." (Police Constable)

In a similar vein, during the Time 2 interviews one in five respondents noted that in their opinion the Hospital had legitimacy within the TS when healthcare workers treated PMIs who were brought in by Police. While most of these respondents just mentioned that ED doctors and psychiatrists treated the PMIs, some were more detailed in their explanation of treatment. Exemplar quotes that typify the first-order code 'Healthcare workers treat PMIs' include the following:

"Get a patient seen as soon as possible and whatever treatment needs to be done to be done as soon as possible." (Hospital Administrator)

"Just giving the treatment to the patients, the doctor sees the patients and we just go through the treatment including RE, bloodwork, fully catheterisation, and all that stuff"' (ED Nurse) "It's part of their job as healthcare people. They're dealing with mental health. It's part of the healthcare system. It's something that... like anybody else comes in with a ... with a health problem or health issue, they as physicians and healthcare professionals have to... have to deal with it and treat it... the best they can." (Police Constable)

Perceptions of Legitimate Police Actions: According to the employees we interviewed post-change, the Police are legitimate stakeholders within the PMI process because they perform 
the following three activities: (1) Police apprehend PMIs under mental health act, (2) Police keep community safe, and (3) Police provide background information about PMI to doctor. During the time 2 interviews, the vast majority (four out of every five) of the respondents mentioned the fact that the Police apprehended the PMIs under the mental health act gave them legitimacy within the PMI process. The following quotes from Time 2 interviews illustrate the type of comments that were grouped under the label 'Police apprehend PMIs under the mental health act':

"The Police have the authority to apprehend you under the Mental Health Act. You can't refuse going to the Hospital if the Police feel that you need to in this kind of situation." (Hospital Resource Nurse)

"Police are one of the only folks under the legislation, therefore the law, to apprehend folks, again involuntarily." (Social Service Agency Frontline Justice)

When asked during Time 2 interviews about legitimacy within the PMI process, one in four respondents spoke to the idea that police officers keep the community safe when they deal with individuals with mental illness who are having a mental health crisis. We gave the descriptor 'Police keep community safety' to respondents' comments that illustrated how police officers may act to keep the community safe. For example:

"If they're actually displaying some sort of mental problem at the time. If they're going through some sort of situation that displays to us that they're not safe to provide for themselves anymore, they're going to require some assistance or care to make sure they are okay and everyone else." (Police Constable)

"Making sure that everybody is safe. I think that's probably the best way of putting it is that I see them [the Police] as the ones who are kind of keeping everybody safe and trying to 
contain the situation kind of contain the person especially if the person is starting to get violent." (Social Service Agency Frontline ACT)

Perceptions of Legitimate Social Service Agency Actions: During the Time 2 interviews, respondents identified three actions taken by employees working for the Social Service Agency that they felt gave these workers legitimacy within the PMI process: (1) Social workers provide information about the PMI to healthcare workers and police officers, (2) Social workers advocate for the PMI during the PMI process, and (3) InSted workers connect PMIs to community services.

First, respondents (cited by $28 \%$ of the respondents) noted that the provision of background information could include (but was not limited to) the PMI's past behavior, previous Hospital visits, diet, and current medication plan. We gave this type of comment the label 'Social workers provide PMI information to Hospital and Police'. Such comments spoke to the idea that community support workers would empower others in the PMI process by passing along information about the PMI. The following two quotes illustrate how community support workers may contribute to the well-being of the PMI by passing information along to the Police or the Hospital:

"So I guess what comes to mind for me for legitimacy is if it is a legitimate call, a legitimate reason to be calling the police. So I would say for me as a worker at [the Social Service Agency] if a client we believe is at risk of harming themselves or others, this makes it a legitimate call. It doesn't matter how, if we think they may follow through or not, if they're saying it we need to give the benefit of the doubt and pass the information along to the appropriate people. And the Police and the other workers involved, the Hospital, the InSted team can sort out all the other pieces, but just bringing it to the attention of the Police makes 
it, or I guess the severity of the situation would make it a legitimate reason to call them." (Social Service Agency Frontline ACT)

"So we've got a client whose baseline is quite psychotic and he calls the Police often because he wants to discuss the bible, it's the middle of the night, there's not that many other resources out there so often the Police bring him to emerg as a mental health apprehension. Because we share a common database with InSted workers, the InSted worker was able, on one of the last kind of attempted apprehensions, to say although he appears psychotic this is his baseline and there's going to be nothing gained by admitting him. It was really great that you didn't tie up a bed and so on and so forth." (Social Service Agency Frontline ACT)

Seventeen percent of respondents interviewed after changes were implemented within the TS also felt that the Social Service Agency employees demonstrated legitimacy by advocating for PMIs to police officers (usually when officers were determining whether to apprehend the PMI), and to healthcare workers (usually when Psychiatrists were determining whether to discharge the PMI from the Hospital). We gave the descriptor 'Social worker advocates for PMIs to Hospital and Police' to this type of comment. For example:

"There are opportunities where InSted is able to advocate for a client in terms of their disposition in the Hospital, so if we were to meet somebody and we were to assess that their needs are different than what was presented to us by the Hospital team, there is an opportunity for us to share that opinion with our team." (Social Service Agency Frontline $\mathrm{ACT})$

"I think we have the voice for clients and expressing their experiences... and sharing them with the Police as well as with the Hospital.” (Social Service Agency Frontline Justice) 
Finally, respondents also felt that the fact that Social Service Agency workers demonstrated legitimacy by taking actions to help the PMI post discharge from the MHESU (cited by $13 \%$ of the respondents). More specifically, they noted that InSted workers would contact a variety of services in the community and determine which community service would be most appropriate for this PMI (e.g. safe beds). This type of comment was labeled 'InSted workers connect PMIs to community services'. For example:

"We can really have a significant input into whether or not they're [PMIs are] connected with our service, or a different service in the community." (Social Service Agency InSted worker)

\subsubsection{PERCEPTIONS OF LEGITIMACY WITHIN THE TS: TS STAKEHOLDER SAMPLE POST-CHANGE (TIME 2)}

Table 50 provides similar information to that which was shown in Table 49, but in this case the data are broken down by TS stakeholder to allow us to determine similarities and differences in perceptions of legitimacy within the TS post implementation of planned changes (i.e. Time 2). Analysis of the second-order categories are reported first, followed by the analysis of first-order codes.

Analysis of the second-order categories data show that, post-change, there were no areas of common agreement at this level of analysis between the three TS stakeholders with respect to how legitimacy was demonstrated within the TS. While the Police and the Social Service Agency strongly shared perceptions of legitimate Police actions, and the Hospital and the Police strongly shared perceptions of legitimate Hospital actions, there were no perceptions of legitimacy commonly held by all three TS stakeholder groups. More specifically, respondents in the Police $(100 \%)$ and Social Service Agency $(85 \%)$ samples shared the view that the Police were 
legitimately involved in the PMI process post change, while respondents in the Hospital (80\%) and Police (70\%) samples shared the view that the Hospital engaged in actions after the planned TS change was implemented that contributed to the perception that they had legitimacy within the TS. Finally, it is interesting to note that those in the Social Service Agency sample uniquely held the view that their actions conferred legitimacy within the PMI process, even though the others did not.

Examination of the first-order codes gives us additional information on how perceptions of legitimacy exhibited 'sharedness' across organizational boundaries within the TS. More specifically, it is noteworthy that a few respondents from each of the three stakeholders mentioned that the police demonstrated legitimacy within the TS by keeping the community safe by taking people with mental illness to the hospital (i.e. weak common perception).

Three shared perceptions of legitimacy could also be observed within the TS after changes were implemented. More specifically, respondents from the Police (100\%) and Social Service Agency $(85 \%)$ strongly shared the perception that police officers demonstrated legitimacy within the TS by apprehending PMIs under the mental health act. Similarly, respondents in the Hospital $(80 \%)$ and Police $(70 \%)$ samples strongly shared the perception that healthcare workers showed legitimacy within the TS when they assessed and treated PMIs after they were brought to the ED.

Finally, it is interesting to note that the Police and the Social Service Agency each held unique perceptions of how they demonstrated their own legitimacy within the TS. The Police provided one of these unique responses, while the other three were given by those in the Social Service Agency sample. More specifically, those in the Police sample weakly held the unique view that they demonstrated their own legitimacy within the PMI process when they provided information to doctors. Social Service Agency workers held the unique views that they acted 
legitimately when they (1) provided PMI information to their partners (strong unique), (2) advocated for the PMI (strong unique), and (3) connected PMIs to community services (weak unique).

The following points summarize our analysis of how respondents perceived legitimacy within the TS after the implementation of the planned TS change:

- All three TS stakeholders weakly agreed that Police keeping the community safe demonstrated legitimacy within the TS.

- The Hospital and Police stakeholders shared two views:

- Healthcare workers assessing PMIs was a strong indicator that the Hospital demonstrated legitimacy within the TS; and,

- Healthcare workers treating PMIs was a weak indicator that the Hospital displayed legitimacy within the TS.

- The Police and Social Service Agency shared the strong view that apprehending PMIs under the mental health act was an indicator of the Police's legitimacy within the TS.

- The Social Service Agency held the unique views that they displayed legitimacy when they:

- Provided PMI information to the Hospital or Police (strong);

- Advocated for PMIs to the Hospital and Police (strong); and,

- Connected PMIs to community services (weak).

- The Police stakeholder expressed the weak view that providing information to the doctor displayed legitimacy within the TS. 


\begin{tabular}{|c|c|c|c|c|c|c|c|}
\hline \multirow{2}{*}{$\begin{array}{c}\text { Perceptions of Legitimacy within the TS: } \\
\text { TS Stakeholder Sample Post-Change } \\
\text { (Time 2) }\end{array}$} & \multicolumn{2}{|c|}{$\begin{array}{l}\text { Hospital } \\
(n=20)\end{array}$} & \multicolumn{2}{|c|}{$\begin{array}{l}\text { Police } \\
(\mathbf{n}=\mathbf{2 0})\end{array}$} & \multicolumn{2}{|c|}{$\begin{array}{l}\text { Social Service } \\
\text { Agency } \\
(\mathbf{n}=\mathbf{2 0})\end{array}$} & \multirow{2}{*}{$\begin{array}{c}\text { Post-Change } \\
\text { Perception } \\
\text { (Common / Shared / } \\
\text { Unique) }\end{array}$} \\
\hline & $\#$ & $\%$ & $\#$ & $\%$ & $\#$ & $\%$ & \\
\hline Perceptions of Legitimate Hospital Actions & 16 & 80 & 14 & 70 & 4 & 20 & Strong Shared (H/P) \\
\hline Healthcare workers assess PMIs & 16 & 80 & 14 & 70 & 4 & 20 & Strong Shared (H/P) \\
\hline Healthcare workers treat PMIs & 5 & 25 & 6 & 30 & 2 & 10 & Weak Shared (H/P) \\
\hline Perceptions of Legitimate Police Actions & 12 & 60 & 20 & 100 & 17 & 85 & Strong Shared (P/SS) \\
\hline Apprehend PMIs under mental health act & 12 & 60 & 20 & 100 & 17 & 85 & Strong Shared (P/SS) \\
\hline Police keep community safe & 5 & 25 & 8 & 40 & 4 & 20 & Weak Common \\
\hline Police provide information to doctor & 1 & 5 & 4 & 20 & 0 & 0 & Weak Unique (P) \\
\hline $\begin{array}{l}\text { Perceptions of Legitimate Social Service Agency } \\
\text { Actions }\end{array}$ & $\mathbf{0}$ & $\mathbf{0}$ & 1 & 5 & 20 & 100 & Strong Unique (SS) \\
\hline $\begin{array}{r}\text { Social workers provide PMI information to Hospital } \\
\text { and Police }\end{array}$ & 0 & 0 & 0 & 0 & 17 & 85 & Strong Unique (SS) \\
\hline Advocate for PMI to Hospital and Police & 0 & 0 & 0 & 0 & 10 & 50 & Strong Unique (SS) \\
\hline InSted connects PMIs to community services & 0 & 0 & 1 & 5 & 7 & 35 & Weak Unique (SS) \\
\hline
\end{tabular}

Note: $\mathrm{H}=$ Hospital; $\mathrm{P}=$ Police; $\mathrm{SS}=$ Social Service Agency.

Table 50: Perceptions of Legitimacy within the TS: TS Stakeholder Sample Post-Change (Time 2) 


\subsubsection{IMPACT OF PLANNED TS CHANGE ON PERCEPTIONS OF LEGITIMACY WITHIN THE TS}

This section presents two complimentary assessments of how the planned TS change may have impacted perceptions of legitimacy within the TS. First, in Section 6.2.5.1, we examine findings using cross-sectional data. The findings from this stage of the analysis will give us a general understanding of how respondents from all three TS stakeholders view legitimacy within the TS. The findings from this analysis also allow us to examine how the planned TS change may have impacted 'sharedness' of perceptions of legitimacy within the TS. It should be noted that the composition of the Time 2 sample is somewhat different from the Time 1 sample, as a result of a high number of people in the Social Service Agency sample withdrawing from the study. This means that, while we are able to make comparisons between TS stakeholders at the group level, we were unable to conduct any analysis at the level of the individual.

Second, in Section 6.2.5.2, we report on the second analysis, which uses panel data. There are strengths and weaknesses with this analysis. While this analysis compares the responses that a particular individual gave at Time 1 (pre-change) to what this person said at Time 2 (postchange), which should allow the researcher to detect how the planned TS change impacted perceptions of legitimacy within the TS, it is limited in that it does not consider data from the Social Service Agency.

Lastly, Section 6.2.5.3 summarizes how planned TS change may have impacted perceptions of legitimacy within the TS. 


\subsubsection{IMPACT OF PLANNED TS CHANGE ON PERCEPTIONS OF LEGITIMACY:}

ANALYSIS OF THE CROSS-SECTIONAL DATA SET

This section seeks to explore how each of the TS stakeholders' views of how legitimacy is demonstrated within the TS may have shifted over time relative to their TS partners' views. To do so, we took a three-pronged approach.

We first examined data describing how respondents felt legitimacy was displayed in the TS before changes were implemented. As shown in Table 48, we classified responses (first-order codes and second-order categories) into three categories: (1) common (all three stakeholders agreed that legitimacy was demonstrated in this manner), (2) shared (two out of three stakeholders agreed that legitimacy was demonstrated in this manner), and (3) unique (only one stakeholder felt that this was a form of legitimacy within the TS). We also categorized these perceptions as being strong $(50 \%$ or more of respondents in each relevant stakeholder group felt this was a form of legitimacy) or weak (less than $50 \%$ of the respondents in each relevant stakeholder group felt this was a form of legitimacy).

We then examined data concerning how legitimacy was demonstrated within the TS six months after the planned change had been implemented from respondents employed at each of the three TS stakeholders. We again classified responses (first-order codes and second-order categories) into three categories: (1) common, (2) shared, and (3) unique. We also categorized these perceptions as being strong or weak as noted above.

Lastly, we examined these data and looked for evidence of shifts in 'sharedness' (see Table 51). We operationally define a shift in 'sharedness' by comparing the classification we gave a perception of legitimacy within the TS at Time 1 (e.g. strong common) with the classification we gave the corresponding perception of legitimacy within the TS at Time 2 (e.g. weak shared). We 
identify three different scenarios using this analysis: (1) no shift in 'sharedness', (2) decreasing 'sharedness' (i.e. more TS stakeholders agreed this was a form of legitimacy at Time 1 than at Time 2), and (3) increasing 'sharedness' (i.e. fewer TS stakeholders agreed that this was a form of legitimacy within the TS at Time 1 than at Time 2). The following section presents data supporting each of these three situations.

No shifts in 'sharedness': Our analysis identified two situations where TS stakeholders did not change their views of legitimacy within the TS. In the first scenario, the planned TS change appears to have had little impact on who thought the Hospital displayed legitimacy within the TS, as their views of Hospital legitimacy did not change over time.

- Both before and after the planned TS change, the Hospital and Police indicated that the Hospital displayed legitimacy within the TS (strong shared) (i.e. Perceptions of Legitimate Hospital Actions).

- The Hospital and the Police mentioned that healthcare workers assessing PMIs was a legitimate act for the Hospital both before and after the planned TS change was introduced (strong shared) (i.e. Healthcare workers assess PMIs).

- Before the implementation of the planned TS change, all three TS stakeholders indicated that healthcare workers treating PMIs was a legitimate action within the TS (weak common) (i.e. Healthcare workers treat PMIs). This sentiment was also shared by Hospital and Police respondents post-change (weak shared between Hospital and Police). The above data support the idea that the planned TS change did not substantially impact the extent that TS stakeholders observed legitimacy within the Hospital. 
Second, our analysis also suggests that the planned TS change had little impact on who thought the Social Service Agency displayed legitimacy within the TS, as respondents' views of Social Service Agency legitimacy did not change over time.

- Both before and after the planned TS change, the Social Service Agency indicated that they displayed legitimacy within the TS (strong unique) (i.e. Perceptions of Legitimate Social Service Agency Actions).

- In Time 1 interviews, the Social Service Agency indicated that community support workers initiating the PMI process was a form of legitimacy within the TS (strong unique) (i.e. Social Service Agency workers initiate PMI process). No respondent had such an observation postchange.

- In Time 1 interviews, respondents from the Social Service Agency stated that, when they supported PMIs in the community, they were demonstrating legitimacy within the TS (weak unique Hospital) (i.e. Social Service Agency worker supports PMI in community). At Time 2, however, no respondent made a similar comment.

- While no respondent mentioned that providing information about the PMI to the Hospital or Police was a legitimate action in the TS before the planned TS change, Social Service Agency respondents did post-change (strong unique) (i.e. Social workers provide PMI information to Hospital and Police).

- In Time 1 interviews, no respondent stated that advocating for PMIs to the Hospital or Police was a legitimate action within the TS (i.e. Social workers advocate for PMI to Hospital and Police). Post-change, however, respondents in the Social Service Agency felt that this was a form of legitimacy in the TS (strong unique). 
- No respondent indicated that there was legitimacy in an InSted worker connecting PMIs to community services pre-change (i.e. InSted workers connect PMIs to community services). Those within the Social Service Agency did, however, suggest that this was a form of legitimacy post-change (weak unique).

It would appear that who perceived legitimacy within the Social Service Agency did not shift as a result of the planned TS change. There was a shift, however, in how community support workers felt they displayed legitimacy within the TS.

Decreasing 'sharedness': While all three TS stakeholders mentioned that the Police displayed legitimacy within the TS at Time 1, only two of the stakeholders indicated that the Police had legitimacy post-change.

- Pre-change all three TS stakeholders felt that the Police had legitimate actions within the TS (strong common) (i.e. Perceptions of Legitimate Police Actions). After the introduction of the planned TS change, however, only the Police and Social Service Agency felt that the Police acted legitimately within the TS (strong shared between Police and Social Service Agency).

- In Time 1 interviews, all three TS stakeholders indicated that Police apprehending PMIs under the Mental Health Act was legitimate (strong common) (i.e. Police apprehend PMI under Mental Health Act). Unfortunately, the respondents in the Hospital did not mention that such an action was legitimate post-change.

These findings suggest that the views of Police legitimacy eroded in the eyes of healthcare workers. More specifically, although before the implementation of any changes all three stakeholders thought that Police apprehending PMIs was a legitimate action, healthcare workers did not report that these were legitimate actions six months after the implementation of the planned TS change.

We summarize these findings to the following two points: 
- The way in which community support workers viewed legitimate actions within the Social Service Agency changed over time.

- Our analysis suggests that the planned TS change did not impact how respondents perceive legitimacy within the Hospital.

- The planned TS change seems to have decreased the extent to which those in the Hospital sample perceive that the Police display legitimacy within the TS. 


\begin{tabular}{|c|c|c|c|}
\hline Perceptions of Legitimacy in the TS Over Time & $\begin{array}{c}\text { Pre-Change } \\
\text { Perception (Common } \\
\text { / Shared / Unique) }\end{array}$ & $\begin{array}{c}\text { Post -Change } \\
\text { Perception } \\
\text { (Common / Shared } \\
\text { / Unique) } \\
\end{array}$ & $\begin{array}{l}\text { Shift in } \\
\text { 'Sharedness' }\end{array}$ \\
\hline Perceptions of Legitimate Hospital Actions & Strong Shared (H, P) & Strong Shared (H/P) & No Shift \\
\hline Healthcare workers assess PMIs & Strong Shared $(\mathrm{H}, \mathrm{P})$ & Strong Shared $(\mathrm{H} / \mathrm{P})$ & No Shift \\
\hline Healthcare workers treat PMIs & Weak Common & Weak Shared $(\mathrm{H} / \mathrm{P})$ & Decrease \\
\hline Perceptions of Legitimate Police Actions & Strong Common & Strong Shared (P/SS) & Decrease \\
\hline Police apprehend PMI under Mental Health Act & Strong Common & Strong Shared $(\mathrm{P} / \mathrm{SS})$ & Decrease \\
\hline Police keep community safe & Weak Common & Weak Common & No Shift \\
\hline $\begin{array}{r}\begin{array}{r}\text { Police provide background information about PMI to } \\
\text { doctors }\end{array} \\
\end{array}$ & Weak Unique $(\mathrm{P})$ & Weak Unique (P) & No Shift \\
\hline Perceptions of Legitimate Social Service Agency Actions & Strong Unique (SS) & Strong Unique (SS) & No Shift \\
\hline Social Service Agency workers initiate PMI process & Strong Unique (SS) & & No Shift \\
\hline Social Service Agency worker supports PMI in community & Weak Unique (SS) & & No Shift \\
\hline $\begin{array}{r}\text { Social workers provide PMI information to Hospital and } \\
\text { Police }\end{array}$ & & Strong Unique (SS) & No Shift \\
\hline Social workers advocate for PMI to Hospital and Police & & Strong Unique (SS) & No Shift \\
\hline InSted workers connect PMIs to community services & & Weak Unique (SS) & No Shift \\
\hline
\end{tabular}

Note. Only responses associated with first-order codes that were given a common, shared, or unique label at Time 1 and/or at Time 2 are shown.

Table 51: Perceptions of Legitimacy within the TS Over Time: Cross-Sectional Data 


\subsubsection{IMPACT OF PLANNED TS CHANGE ON PERCEPTIONS OF LEGITIMACY:}

\section{ANALYSIS OF THE PANEL DATA SET}

Table 52 presents data relating to changes to the perceptions of legitimacy within the TS over time by using panel data to compare what a respondent said at Time 1 to what that same respondent said at Time 2 . Within our analysis of the panel data, we consider a change to a perception of legitimacy within the TS has occurred when a substantive number of respondents gave a response either: (1) at Time 1 but not at Time 2, or (2) at Time 2 but not at Time 1 . We arbitrarily define a substantive difference between Time 1 and Time 2 responses as a difference of 5 respondents per TS stakeholder (i.e. $25 \%$ of a TS stakeholder sample).

It should be noted that only those perceptions of legitimacy that met our criteria for a substantive difference between Time 1 and Time 2 are shown in Table 52. A full analysis of all perceptions of legitimacy using the panel data set is found in the Appendix.

\begin{tabular}{|c|c|c|c|}
\hline & $\begin{array}{l}\text { Perception } \\
\text { mentioned }\end{array}$ & $\begin{array}{c}\text { Hospital } \\
(\mathbf{n}=\mathbf{2 0})\end{array}$ & $\begin{array}{l}\text { Police } \\
(\mathbf{n}=\mathbf{2 0})\end{array}$ \\
\hline \multicolumn{4}{|l|}{$\begin{array}{l}\text { Perceptions of Legitimate Police } \\
\text { Actions }\end{array}$} \\
\hline \multirow{2}{*}{$\begin{array}{r}\text { Police apprehend PMI under } \\
\text { Mental Health Act }\end{array}$} & $\begin{array}{l}\text { Said T1 } \\
\text { not T2 }\end{array}$ & $\begin{array}{c}6 \\
(30 \%) \\
\end{array}$ & $\begin{array}{c}0 \\
(0 \%) \\
\end{array}$ \\
\hline & $\begin{array}{l}\text { Said T2 } \\
\text { not T1 }\end{array}$ & $\begin{array}{c}1 \\
(5 \%) \\
\end{array}$ & $\begin{array}{c}1 \\
(5 \%) \\
\end{array}$ \\
\hline \multicolumn{4}{|l|}{$\begin{array}{l}\text { Perceptions of Legitimate } \\
\text { Hospital Actions }\end{array}$} \\
\hline \multirow{2}{*}{ Healthcare workers assess PMIs } & $\begin{array}{l}\text { Said T1 } \\
\text { not T2 }\end{array}$ & $\begin{array}{c}7 \\
(35 \%)\end{array}$ & $\begin{array}{c}2 \\
(10 \%)\end{array}$ \\
\hline & $\begin{array}{l}\text { Said T2 } \\
\text { not T1 }\end{array}$ & $\begin{array}{c}6 \\
(30 \%)\end{array}$ & $\begin{array}{c}1 \\
(5 \%)\end{array}$ \\
\hline
\end{tabular}

Note. $\mathrm{T} 1=$ Time $1 . \mathrm{T} 2=$ Time 2 . Shaded cells identify substantive changes in perceptions of legitimacy.

Table 52: Perceptions of Legitimacy within the TS Over Time: Representative Panel Data 
Once we identified a change in perception of legitimacy over time, we conducted followup analysis by examining the comments made by individuals who we classified as having a changed view. This follow-up analysis specifically looked for patterns emerging from the data that could explain how the planned TS change may have impacted views of legitimacy over time.

Based on our analysis of the results in Table 52, the planned TS change appears to have negatively impacted healthcare workers' views that the Police displayed legitimacy within the TS. More specifically, the percent of respondents in the Hospital sample who said that the Police behaved legitimately when they apprehended PMIs under the mental health act declined by $30 \%$ over time. When we turned to the comments that those 6 healthcare workers made in order to get a better understanding why their views changed over time, we noticed one pattern emerging from the data. These six healthcare workers all mentioned that the Police were bringing in more PMIs who may or may not have had a diagnosable mental illness after the planned TS change. One ED resource nurse commented: "I think the Police are bringing in more people who are very mild and not that severe..." This finding is somewhat consistent with data found in Table 16, which show that there has been an increase to the percent of PMIs apprehended in the community. Upon further investigation, one Police manager shines light on why police officers may be apprehending more PMIs with "milder" forms of mental illness.

"I think because our officers are becoming more educated with recognizing legitimate mental health concerns... I think there are a lot of people out there with legitimate mental health concerns and we're clearly not psychologists or psychiatrists out here and we're out here trying to recognize some of these symptoms so I think it has impacted that and allowed us a better understanding of diagnosing some of these issues for the doctors." 
The above comment suggests that Police are apprehending more PMIs because their training (enhanced mental health training for officers was also part of the planned TS change) has allowed them to better identify individuals with legitimate mental health concerns. Unfortunately, the fact that Police are now identifying and apprehending individuals with mild mental health concerns, according to 6 healthcare workers, has meant that healthcare workers view the Police displaying less legitimacy within the TS.

The planned TS change also seems to have contributed to confusion within the Hospital with respect to their own legitimacy. For example, one group of 7 healthcare workers thought they displayed legitimacy by assessing PMIs only before the implementation of the planned TS change, whereas a different group of 6 different healthcare workers thought this was a form of legitimacy only after the planned TS change was implemented. Examination of the comments that individuals made within each of these two subgroups of the Hospital sample reveals that these differing views of Hospital legitimacy may be related to the implementation of the intervention and escalation policy, which has resulted in healthcare workers assessing PMIs quicker. The healthcare workers who thought the PMI's assessment was a legitimate action only before the planned TS change discussed during their Time 2 interviews spoke about the rush to assess PMIs, and how this rush may have led to inappropriate forming.

"I think in the rush to assess the people sometimes you see people who wouldn't normally get formed get placed on a form and this could be to do with when the police are here it gives the staff time to observe their behaviours. Going from a six-hour observation, not that it was good for the Police, down to a two-hour observation, there might be a lot more question and uncertainty whether or not the patient really needs to be here and at that point might be placed on a form and directed to psychiatry just because they are showing some 
symptoms, but not really able to fully observe it and document that patient's behaviour." (Hospital Resource Nurse)

"I think that the only reason why the forming rate is going up is because the doctors are simply forming the patient to expedite the Police to get out." (Hospital Nurse)

The above comments suggest that there are more PMIs are getting formed after the planned TS change than before the planned TS change. This finding is consistent with Table 17, which uses quantitative data to show that the percentage of PMIs getting formed has increased over time.

On the other hand, the healthcare workers who thought that the PMI's assessment was a legitimate action only after the planned TS change tended to speak about the doctor assessing the PMIs quicker from a positive perspective. A Hospital triage nurse commented: "We are providing the help they need quicker."

We summarize our analysis of panel data with the following two points:

- The change associated with the Police mental health training may have resulted, somewhat counter intuitive, in fewer healthcare workers perceiving that the Police displays legitimacy within the TS.

- The implementation of the intervention and escalation policy appears to have impacted healthcare workers' views of their own legitimacy in two ways:

- One subgroup of healthcare workers views the PMI's assessment as more legitimate; and,

- Another subgroup of healthcare workers views the PMI's assessment as less legitimate. 


\subsubsection{SUMMARY OF HOW THE PLANNED TS CHANGE MAY HAVE IMPACTED PERCEPTIONS OF LEGITIMACY}

In Section 6.1.5, we explored changes to perceptions of legitimacy within the TS in order to get a better understanding of how the planned TS change may have impacted TS stakeholder legitimacy. Our complimentary analyses (see Section 6.2.5.1 and Section 6.2.5.2) shows that the planned TS change has impacted only healthcare workers' perceptions of legitimacy within the TS.

Our analysis suggest that the planned TS change decreased Police legitimacy, particularly in the eyes of healthcare workers. The changes to these views of the healthcare workers in our sample may be indirectly attributed to the increased mental health training for police officers. Our analysis suggests this increase in training may have contributed to police officers identifying and then apprehending more PMIs. Unfortunately, the healthcare workers in the sample often felt that a number of the PMIs who were being brought into the Hospital post-change did not need to be brought to the ED. This situation may have resulted in the finding that post-change healthcare workers were less likely to perceive the act of apprehending a PMI and bringing them to the Hospital a legitimate activity.

There was also evidence in the data that supported the notion that the intervention and escalation policy impacted the views of healthcare workers regarding their own legitimacy in assessing PMIs. More specifically, one subgroup of healthcare workers thought that the intervention and escalation policy meant that doctors needed to rush their assessment of the PMI in order to get Police out of the Hospital quicker, which resulted in more PMIs getting inappropriately formed (i.e. associated this change with a decline in legitimacy). In contrast, another subgroup of healthcare workers thought that the reduced time that PMIs needed to wait 
for the doctor's assessment provided the PMI with an improved experience (i.e. received care quicker), which they associated with increased legitimacy for the Hospital.

\subsection{PERCEPTIONS OF URGENCY WITHIN THE TS}

The presentation of findings associated with perceptions of TS stakeholder urgency are broken down as follows. The presentation of findings associated with perceptions of urgency within the TS using the cross-sectional data set is presented in the first four subsections. We first present perceptions of urgency within the TS prior to the implementation of the planned TS change for the total sample (Section 6.3.1), and then by TS stakeholder (Section 6.3.2). In Section 6.3.3, we present Time 2 findings for the total sample (Section 6.3.3). Section 6.3.4 presents an examination of these post-change findings at the level of the TS stakeholder. The final section, Section 6.3.5, explores how the planned TS change may have impacted TS stakeholder urgency by examining both cross-sectional and panel data showing changes to perceptions of urgency over time.

\subsubsection{PERCEPTIONS OF URGENCY WITHIN THE TS: TOTAL SAMPLE PRE- CHANGE (TIME 1)}

Analysis of the Time 1 interviews identified six actions that respondents discussed in terms of urgency within the TS before the introduction of the planned TS change (i.e. first-order codes). Two of these first-order codes relate to ways in which respondents view the Police's urgency within the TS and were grouped together within a second-order category entitled: Perceptions of Urgent Police Actions. Two of these first-order codes were associated with ways in which respondents view the Hospital's urgency within the TS and were grouped together within a secondorder category labeled: Perceptions of Urgent Hospital Actions. The two remaining first-order codes relate to respondents' views of Social Service Agency urgency within the TS and formed 
the second-order category: Perceptions of Urgent Social Service Agency Actions. All first-order codes are grouped with their respective second-order category and displayed in Table 53, in descending order by respondent count. Although they are included in Table 53, one Hospital action and one Social Service Agency action that were identified by respondents as typifying how these stakeholders acted urgently within the TS were mentioned by less than $10 \%$ of the total sample and will not be discussed in this section.

\begin{tabular}{|c|c|c|}
\hline \multirow{2}{*}{$\begin{array}{l}\text { Perceptions of Urgency within the TS: } \\
\text { Total Sample Pre-Change (Time 1) }\end{array}$} & \multicolumn{2}{|c|}{$\begin{array}{c}\text { Total } \\
(n=75)\end{array}$} \\
\hline & \# & $\%$ \\
\hline Perceptions of Urgent Hospital Actions & 11 & 15 \\
\hline Physicians need to assess PMIs in a timely manner & 11 & 15 \\
\hline $\begin{array}{r}\text { Other Hospital Urgency (i.e. medicate PMI, } \\
\text { discharge PMI) }\end{array}$ & 3 & 4 \\
\hline Perceptions of Urgent Police Actions & 73 & 98 \\
\hline Police leave Hospital in a timely manner & 68 & 91 \\
\hline Police transport PMI to Hospital in a timely manner & 60 & 78 \\
\hline $\begin{array}{l}\text { Perceptions of Urgent Social Service Agency } \\
\text { Actions }\end{array}$ & 8 & 11 \\
\hline $\begin{array}{r}\text { Community support worker calls } 911 \text { to request } \\
\text { police assistance with high risk PMI }\end{array}$ & 8 & 11 \\
\hline $\begin{array}{r}\text { Community support worker locates PMI after } \\
\text { Hospital discharge }\end{array}$ & 4 & 5 \\
\hline
\end{tabular}

Table 53: Perceptions of Urgency within the TS: Total Sample Pre-Change (Time 1)

Second-Order Categories: When we asked employees what actions in the PMI process they consider urgent, almost everyone ( $98 \%$ of total sample) identified actions taken by the Police. About one in ten of the respondents talked about things that the Hospital (15\% of total sample) or the Social Service Agency (11\% of total sample) did that suggested urgency. Analysis of the firstorder codes helps us understand why this might be the case.

Perceptions of Urgent Hospital Actions: Respondents indicated only one type of action performed by healthcare workers that they thought had urgency within the TS. More specifically, only $15 \%$ of the respondents noted that there were, at times, a rush for the physician to assess the 
PMI. This type of comment was coded as 'Physicians need to assess PMIs in a timely manner'.

The following quotes illustrate the Hospital urgency between the time the PMI arrives at the Hospital until the time they are seen by a physician:

"Once the patient is brought to the Hospital I think there is a time sensitivity both out of consideration for the resources of the Police, allow them to be back on the street, and also out of consideration for the patient. I mean the patient may very well be apprehended against their will, and have rights to freedom of movement really. So I think that there is some urgency to think about police and the patient, and to make a decision as to whether or not the patient can be released." (Hospital Administrator)

"If the person overdosed or they've taken a whole bunch of medication, then usually once you actually get to the Hospital, they are a little better... the person gets assessed right away, just to make sure that everything's okay inside their bodies, and that's really the time sensitive-stuff right there." (Police Constable)

"If the person has been expressing suicidal or homicidal thoughts, there's an urgency to get them help right away. That's the biggest issue, especially if they're a harm to themselves or others. Or again, if their health is rapidly deteriorating because, let's say, with the insulin, they have diabetes, they're not taking their insulin properly, and they're not managing their physical medications properly, that could be again a life and death issue that requires a real time-sensitive intervention to get them into a stable environment. They need to be in front of a doctor." (Social Service Agency Frontline ACT)

Perceptions of Urgent Police Actions: According to the employees we interviewed, there were two urgent actions that were performed by police officers within the PMI process: (1) Police leave Hospital in a timely manner, and (2) Police transport PMI to Hospital in a timely manner. 
The most frequently mentioned Police activity that was considered urgent was given the label 'Police leave Hospital in a timely manner'. Nearly all respondents ( $91 \%$ of total sample) commented on the amount of time that police officers had to wait at the Hospital for their detainee to be assessed by a doctor before they could return to their other duties. The following quotes illustrate the time sensitivity associated with the actions included within this first-order code: "I believe the wait period is probably the most time sensitive... Especially overnight, if the Police are bringing somebody around, let's say 20h00, that patient won't be assessed until the next following day. So sometimes they're sitting in emerge, especially if MHESU's full, for 16 to 24 hours." (Hospital Security Guard)

"Waiting in the Hospital is problematic for the individual we've apprehended and for the Police, as an agency that has other responsibilities. Most of the time, I've got to wait eight to twelve hours just to talk to a doctor." (Police Constable)

"I would say Police waiting, because they have a number of duties that they have to do during a shift, and if they're held down eight hours in a waiting room for somebody to be assessed, that's going to take them off the road." (Social Service Agency Frontline Justice)

The urgent Police action that was mentioned the second most frequently by employees interviewed at Time 1 was given the descriptor 'Police transport PMI to Hospital in a timely manner'. Three-quarters of the sample ( $78 \%$ of total sample) discussed the importance of the Police rushing to the mental health crisis, quickly and effectively intervening with the PMI, and escorting that person to the Hospital if deemed appropriate. Illustrative quotes for this urgent action are provided below: 
"By definition the Police actions are time-sensitive... if there's no urgency to act, there's no perceived risk to anybody, then the Police should not be apprehending." (Hospital MHESU Manager)

"I think the initial assessment done by Police at the time of the call or the time of the arrival at the scene is time sensitive. Situations with an EDP person, and their behaviours, can change pretty rapidly. It can be intense." (Police Constable)

"A response by the Police is very urgent. In a case I just had this last week, she didn't suspect what was happening. She had no idea. Because it happened so quickly we were able to get her the help that she needed. The timely response of the Police was excellent. I called them and they said be there because we're going to be there. They were there in less than five minutes. So, you know, like, just really fast, boom, boom, boom." (Social Service Agency Frontline ACT)

"Probably getting them to the Hospital, that's very urgent, that's very time-sensitive, and that can be difficult because clients can be hard to find, and unwilling to go, so I understand that using Police might be the quickest method of getting them to their treatment." (Social Service Agency Frontline ACT)

Perceptions of Urgent Social Service Agency Actions: Analysis of the data identified only one urgent action for the Social Service Agency in the PMI process: calling 911. We labelled this type of perceived urgency: 'Community support worker calls 911 to request Police assistance with high risk PMI'. Eight out of the 75 respondents (11\% of total sample) at Time 1 commented that there was time sensitivity for social workers to call 911. For example:

"It is clearly mentioned in the Mental Health Act that when a person is at risk to himself or the community, it is our duty not only as a mental health worker but as a citizen of this 
country that we should inform Police about it so Police can take the person to the Hospital for the treatment. So with situations when a person is feeling paranoid and he thinks everybody is against him and he has no understanding of what is going on in his surroundings, we have to call the police and sometimes we don't have a lot of time." (Social Service Agency Frontline ACT)

\subsubsection{PERCEPTIONS OF URGENCY WITHIN THE TS: TS STAKEHOLDER SAMPLE PRE-CHANGE (TIME 1)}

Table 54 provides similar information to that which was shown in Table 53, but in this case the data are broken down by TS stakeholder to allow us to determine similarities and differences in perceptions of urgency within the TS before changes were implemented (i.e. Time 1). Analysis of the second-order categories are reported first, followed by the analysis of first-order codes.

There were three second-order categories related to perceptions of urgency within the TS before changes were implemented. Our analysis classified one of them as a common perception, given that all but 2 out of 75 respondents mentioned Police actions that they thought were urgent (i.e. strong common perception).

The other two second-order categories met our criteria for weak unique perception. In particular, primarily healthcare workers ( $36 \%$ of Hospital sample) mentioned at least one Hospital action they considered urgent, while community support workers (32\% of Social Service Agency sample) were the only respondents to mention at least one Social Service Agency action they considered urgent. 


\begin{tabular}{|c|c|c|c|c|c|c|c|}
\hline \multirow{2}{*}{$\begin{array}{c}\text { Perceptions of Urgency within the TS: } \\
\text { TS Stakeholder Sample Pre-Change } \\
\text { (Time 1) }\end{array}$} & \multicolumn{2}{|c|}{$\begin{array}{c}\text { Hospital } \\
(\mathrm{n}=\mathbf{2 5})\end{array}$} & \multicolumn{2}{|c|}{$\begin{array}{l}\text { Police } \\
(\mathrm{n}=25)\end{array}$} & \multicolumn{2}{|c|}{$\begin{array}{c}\text { Social Service } \\
\text { Agency } \\
(\mathbf{n}=\mathbf{2 5})\end{array}$} & \multirow{2}{*}{$\begin{array}{c}\text { Pre-Change Perception } \\
\text { (Common / Shared / } \\
\text { Unique) }\end{array}$} \\
\hline & $\#$ & $\%$ & $\#$ & $\%$ & $\#$ & $\%$ & \\
\hline Perceptions of Urgent Hospital Actions & 9 & 36 & 1 & 4 & 1 & 4 & Weak Unique (H) \\
\hline Physicians need to assess PMIs in a timely manner & 9 & 36 & 1 & 4 & 1 & 4 & Weak Unique $(\mathrm{H})$ \\
\hline Perceptions of Urgent Police Actions & 24 & 96 & 25 & 100 & 24 & 96 & Strong Common \\
\hline Police leave Hospital in a timely manner & 24 & 96 & 24 & 96 & 20 & 80 & Strong Common \\
\hline Police transport PMI to Hospital in a timely manner & 13 & 52 & 25 & 100 & 22 & 88 & Strong Shared (P, SS) \\
\hline $\begin{array}{l}\text { Perceptions of Urgent Social Service Agency } \\
\text { Actions }\end{array}$ & $\mathbf{0}$ & $\mathbf{0}$ & $\mathbf{0}$ & $\mathbf{0}$ & 8 & 32 & Weak Unique (SS) \\
\hline $\begin{array}{r}\text { Community support worker calls } 911 \text { to request } \\
\text { police assistance with high risk PMI }\end{array}$ & 0 & 0 & 0 & 0 & 8 & 32 & Weak Unique (SS) \\
\hline
\end{tabular}

Note: $\mathrm{H}=$ Hospital; $\mathrm{P}=$ Police; $\mathrm{SS}=$ Social Service Agency.

Table 54: Perceptions of Urgency within the TS: TS Stakeholder Sample Pre-Change (Time 1) 
Further, analysis of the four first-order codes revealed the following. One of the first-order codes was classified as a strong common perception. More specifically, nearly all respondents in our pre-change sample mentioned that they thought police officers leaving the Hospital in a timely manner reflected urgency for the Police.

Second, analysis of the data found in Table 54 reveals one shared perception of urgency within the TS prior to the implementation of changes. Police officers (100\% of Police sample) and social workers ( $88 \%$ of Social Service Agency sample) who were interviewed at Time 1 strongly shared the perception that transporting the PMI to the Hospital was an urgent action within the TS.

Finally, our analysis of first-order codes identified two weakly held unique perceptions of urgency. Interestingly, both of these cases involved respondents perceiving their own TS stakeholder acting urgently in ways that did not seem to be as obvious to those employed at other TS stakeholders. More specifically, healthcare workers (36\% of Hospital sample) weakly held the unique perception that physicians assessing PMIs in a timely manner was an urgent action for the Hospital, while social workers (32\% of Social Service Agency sample) weakly held the unique perception that community support workers calling 911 to request Police assistance with a high risk PMI was an urgent action for the Social Service Agency.

The following points summarize our analysis of what respondents thought were urgent actions within the TS before the planned TS change was implemented:

- All TS stakeholders strongly held the common view that Police leaving the Hospital in a timely manner was an urgent action.

- The Police and Social Service Agency strongly shared the view that Police transporting the PMI to the Hospital was an urgent action. 
- The Hospital expressed the weak view that they acted urgently when physicians assessed PMIs in a timely manner.

- The Social Service Agency expressed the weak view that they acted urgently when they called 911 to request Police assistance.

\subsubsection{PERCEPTIONS OF URGENCY WITHIN THE TS: TOTAL SAMPLE POST- CHANGE (TIME 2)}

Data analysis of the Time 2 interviews identified six actions that respondents discussed in terms of urgency within the TS (i.e. first-order codes). Two of these first-order codes were associated with ways in which respondents view the Hospital's urgency within the TS and were grouped together within a second-order category labeled: Perceptions of Urgent Hospital Actions. Two of these first-order codes relate to ways in which respondents view the Police's urgency within the TS and were grouped together within a second-order category entitled: Perceptions of Urgent Police Actions. The two remaining first-order codes relate to respondents' views of Social Service Agency urgency within the TS and formed the parent group: Perceptions of Urgent Social Service Agency Actions. All first-order codes are grouped with their respective second-order category and displayed in Table 55, in descending order by respondent count. Although they are included in Table 55, two actions that were identified by respondents as typifying how these TS stakeholders acted urgently within the TS were mentioned by less than $10 \%$ of the total sample and will not be discussed in this section. In the section below, findings concerning second-order categories are discussed first, followed by our analysis of the first-order codes. 


\begin{tabular}{|c|c|c|}
\hline \multirow{2}{*}{$\begin{array}{c}\text { Perceptions of Urgency within the TS: } \\
\text { Total Sample Post-Change (Time 2) }\end{array}$} & \multicolumn{2}{|c|}{$\begin{array}{c}\text { Total } \\
\text { (n=60) }\end{array}$} \\
\cline { 2 - 4 } Perceptions of Urgent Hospital Actions & 39 & $\mathbf{6 5}$ \\
\hline Physicians need to assess PMIs in a timely manner & 29 & 48 \\
\hline Physicians discharge PMIs in a timely manner & 10 & 17 \\
\hline Perceptions of Urgent Police Actions & $\mathbf{3 0}$ & $\mathbf{5 0}$ \\
\hline Police transport PMI to Hospital in a timely manner & 20 & 33 \\
\hline Police leaving Hospital in a timely manner & 13 & 22 \\
\hline $\begin{array}{c}\text { Perceptions of Urgent Social Service Agency } \\
\text { Actions }\end{array}$ & $\mathbf{4}$ & 7 \\
\hline $\begin{array}{c}\text { Community support worker connects the PMI with } \\
\text { community services after Hospital discharge }\end{array}$ & 4 & 7 \\
\hline Community support worker calls 911 to request \\
Police assistance with high risk PMI
\end{tabular}

Table 55: Total Post-Change Perceptions of Urgency within the TS

Second-Order Categories: Examination of the second-order categories shows that, after the introduction of the planned TS change (see Table 55), two thirds of the respondents indicated that healthcare workers were acting urgently within the PMI process (65\% of total sample). Approximately half of the total sample noted that police officers had urgent behaviors within the PMI process (50\% of total sample). Less than one in ten of the respondents indicated urgent behaviors within the PMI process for the Social Service Agency (7\% of total sample). Analysis of the first-order codes may give us a better understanding why this is the case.

Perceptions of Urgent Hospital Actions: The respondents interviewed post-change identified two urgent actions for the Hospital: one related to physicians assessing incoming PMIs, and the other related to physicians discharging outgoing PMIs (to make room for new PMIs). During post-change interviews, respondents frequently talked about how ED physicians were now urgently working to assess the PMI in a timely manner so that the Police could go back to their other duties on the road. We gave the descriptor 'physicians need to assess PMIs in a timely manner' to comments that suggested the time sensitive nature of physicians assessing PMIs entering the 
ED post-change. Approximately half of the respondents at Time 2 made comments similar to those below:

"It starts pretty much immediately after they get here... a room is expedited, crisis is called if they can be implemented at that time and then we notify the physician that Police, in particular, are with this patient." (Hospital Resource Nurse)

"The length of wait in emerge for patients to be seen by a doctor. That is definitely time sensitive. Patients should be seen within a reasonable time frame and reasonable I guess is left to interpretation. However, I don't think that reasonable would mean three [or] four hours after the patient has arrived and more. I think that is an unreasonable time frame." (Hospital Psychiatrist)

"When the individual is brought in [to the Hospital], I think that everyone needs to be aware and this person needs to be seen immediately. They need to have access to services right away, so that you can really reduce the crisis or their stress that's causing the crisis." (Social Service Agency Frontline ACT)

After changes were implemented, almost one in five respondents related urgency to the need to move the PMI from their current location in the ED to either somewhere else in the Hospital (e.g. psychiatry ward) or out of the Hospital. 'Physicians discharge PMIs in a timely manner' was the code given to this type of comment. Quotes illustrating the meaning of this first-order code are provided below:

"So for downstream and MHESU and the InSted people, the urgency is more once the decision is made that they're not going to be admitted to get them out the door with [community] services." (Hospital Manager) 
"There seems to be a lot of urgency to get them out [of the Hospital] because of limited bed space. I've seen a number of people discharged probably before they're really ready to be discharged. I've seen a few times where they'll try out a new regimen of medication but they don't stay at the hospital long enough to see if it's actually working or not. I meant they check to see if the person is stable and not a risk to themselves or others but whether it's actually going to be effective in the long term... Sometimes these medications take a while to work and often times people are discharged before you really know whether they're going to work or not." (Social Service Agency Frontline ACT)

Perceptions of Urgent Police Actions: When asked what actions in the PMI process were considered urgent after changes were implemented to the PMI process, half of the respondents identified at least one of the following two activities conducted by police officers: (1) transporting the PMI to the Hospital in a timely manner, and (2) leaving the Hospital in a timely manner. Of these two, more respondents mentioned the fact that Police had to transport the PMI to the Hospital in a timely manner. A third of the employees that we interviewed mentioned the urgent nature of Police attending the scene, assessing the situation, making the determination whether to apprehend the individual, and then (potentially) apprehending the PMI and bringing them to the Hospital. For example:

"If there's a crisis we need to get there right away. So probably that's an emergency call, we'll go straight there, so there's definitely an urgency to get there, to see what's going on, to do that evaluation quickly, and see what's happening, before somebody gets hurt, and after that it would come down to resources. The urgency to free up as much resources as we could to go do other police work once we have that person safely at the hospital." (Police Sergeant) 
"I would say the part of the initial interaction from me, so if I notice that the individual needs to be hospitalized it is really important for the police to response in a timely manner because whenever an individual is experiencing psychosis it all depends on what type they are experiencing. It could be anything so, you know, if they are aggressive it would be good if the police could be prompt in terms of their response because they can become extremely unpredictable. I think that action of the response to the call is I think the most important one." (Social Service Agency Frontline ACT)

In the post-change interviews, respondents also noted the urgent nature of police officers leaving the Hospital and returning to their duties in the community. The descriptor 'Police leave Hospital in a timely manner' was given to comments that spoke to the idea that while Police were waiting, they were unable to perform other necessary duties. Approximately a quarter of the respondents that were interviewed after changes were implemented made comments similar to those below:

"Urgency for the Police... they have an urgency to get relieved, have the Hospital provided the service so they can return to the community and the urgency with that is that there are other calls pending and there's other people needing assistance, and if they're here and tied up unable to leave, they're unable to fulfil the community's needs." (Hospital Resource Nurse)

"Urgency in regards to us getting back out onto the road. Urgency from our perspective, is... the city's coming to an end out here. We need the officers on the road. We're in the Hospital and we're... it's been termed as babysitting and I don't want to minimalize what the officers have to do... take away from the struggles that people in crisis might have, but 
sort of tantamount to babysitting because they haven't been seen by a doctor yet and we can't really do anything with them, we're just sitting there babysitting." (Police Constable)

\subsubsection{PERCEPTIONS OF URGENCY WITHIN THE TS: TS STAKEHOLDER SAMPLE POST-CHANGE (TIME 2)}

Table 56 shows first-order codes and second-order categories representing the various postchange perceptions of urgency within the TS by TS stakeholder. Analysis of second-order categories are reported first, then followed by the analysis of first-order categories.

Findings show that there were three second-order categories related to perceptions of urgency within the TS after changes were implemented. The second-order category "Perceptions of Urgent Hospital Actions" was classified as a strong common perception, as the majority of respondents from each TS stakeholder mentioned at least one urgent action for the Hospital after changes were implemented. One of the second-order categories was a strongly shared perception between the TS stakeholder who was perceived to have urgent actions and another TS stakeholder, since respondents from the Police (60\% of Police sample) and Social Service Agency (60\% of Social Service Agency sample) samples noted at least one urgent action for the Police after changes were implemented. Finally, the final second-order category, related to perceptions of urgent Social Service Agency actions, was classified as a weak unique perception, given that community support workers ( $20 \%$ of Social Service Agency sample) were the only group of respondents to mention at least one urgent action for the Social Service Agency after changes were implemented.

Our analysis of the first-order codes identified one as a strong common perception of urgency within the TS. More specifically, at least half of the respondents from each TS stakeholder group mentioned that physicians assessing PMIs in a timely manner was an urgent action for the Hospital. 
There were three shared perceptions of urgency within the TS after changes were implemented. Data associated with all three of these shared perceptions suggest that respondents from two TS stakeholders can perceive ways a TS stakeholder acts urgently within the TS more than respondents employed at the (third) partner organization. For example, respondents from the Police (40\% of Police sample) and the Social Service Agency (60\% of Social Service Agency sample) strongly shared the perception that transporting the PMI to the Hospital in a timely manner was an urgent action for the Police after changes were implemented. Hospital (25\% of Hospital sample) and Social Service Agency (25\% of Social Service Agency sample) respondents weakly shared the perception that discharging PMIs was an urgent action for the Hospital after changes were implemented. Healthcare workers ( $25 \%$ of Hospital sample) and police officers ( $30 \%$ of Police sample) interviewed after changes were implemented weakly shared the perception that Police leaving the Hospital in a timely manner was an urgent action for the Police. 


\begin{tabular}{|c|c|c|c|c|c|c|c|}
\hline \multirow{2}{*}{$\begin{array}{c}\text { Perceptions of Urgency within the TS: } \\
\text { TS Stakeholder Sample Post-Change } \\
\text { (Time 2) }\end{array}$} & \multicolumn{2}{|c|}{$\begin{array}{l}\text { Hospital } \\
(n=20)\end{array}$} & \multicolumn{2}{|c|}{$\begin{array}{l}\text { Police } \\
(\mathbf{n}=\mathbf{2 0})\end{array}$} & \multicolumn{2}{|c|}{$\begin{array}{l}\text { Social Service } \\
\text { Agency } \\
(\mathbf{n}=\mathbf{2 0})\end{array}$} & \multirow{2}{*}{$\begin{array}{c}\text { Post-Change } \\
\text { Perception } \\
\text { (Common / } \\
\text { Shared / Unique) }\end{array}$} \\
\hline & $\#$ & $\%$ & \# & $\%$ & $\#$ & $\%$ & \\
\hline Perceptions of Urgent Hospital Actions & 16 & 80 & 11 & 55 & 15 & 75 & Strong Common \\
\hline Physicians need to assess PMIs in a timely manner & 16 & 80 & 11 & 55 & 10 & 50 & Strong Common \\
\hline Physicians discharge PMIs in a timely manner & 5 & 25 & 0 & 0 & 5 & 25 & Weak Shared (H, SS) \\
\hline Perceptions of Urgent Police Actions & 6 & 30 & 12 & 60 & 12 & 60 & $\begin{array}{c}\text { Strong Shared (P, } \\
\text { SS) }\end{array}$ \\
\hline Police transport PMI to Hospital in a timely manner & 3 & 15 & 8 & 40 & 12 & 60 & Strong Shared $(\mathrm{P}, \mathrm{SS})$ \\
\hline Police leaving Hospital in a timely manner & 5 & 25 & 6 & 30 & 2 & 10 & Weak Shared (H, P) \\
\hline $\begin{array}{l}\text { Perceptions of Urgent Social Service Agency } \\
\text { Actions }\end{array}$ & $\mathbf{0}$ & $\mathbf{0}$ & $\mathbf{0}$ & $\mathbf{0}$ & 4 & 20 & Weak Unique (SS) \\
\hline $\begin{array}{l}\text { Community support worker connects the PMI with } \\
\text { community services after Hospital discharge }\end{array}$ & 0 & 0 & 0 & 0 & 4 & 20 & Weak Unique (SS) \\
\hline
\end{tabular}

Note: $\mathrm{H}=$ Hospital; $\mathrm{P}=$ Police; $\mathrm{SS}=$ Social Service Agency.

Table 56: Perceptions of Urgency within the TS: TS Stakeholder Sample Post-Change (Time 2) 
Lastly, there was one unique perception of urgency within the TS after changes were implemented. This view involved respondents (in this case Social Service Agency employees) mentioning their own TS stakeholder's urgent behaviors. More specifically, one in five of those in the Social Service Agency sample reported that community support workers connecting the PMI with community services after Hospital discharge was an urgent action for the Social Service Agency. We labeled this first-order code 'Community support worker connects the PMI with community services after Hospital discharge'. One community support workers described this urgent action by saying: "Connecting them [the PMI] with the appropriate service in the community, and ideally, even that [community support] worker [responsible for the PMI] would follow up right at the time of discharge would be urgent to me." (Social Service Agency Frontline $\mathrm{ACT})$.

The following points summarize our analysis of how respondents thought urgency was displayed in the TS after the planned TS change was implemented:

- All TS stakeholders strongly agreed that the Hospital acted urgently when physicians assessed PMIs in a timely manner.

- The Police and Social Service Agency stakeholders shared the strong view that the Police act urgently when they transport a PMI to the Hospital.

- The Hospital and the Police stakeholders shared the weak view that the Police had urgency when they were waiting to leave the Hospital.

- The Hospital and Social Service Agency shared the weak view that physicians discharging PMIs in a timely manner was an urgent action.

- The Social Service Agency expressed the weak view that connecting PMIs with community services after Hospital discharge was an urgent action. 


\subsubsection{IMPACT OF PLANNED TS CHANGE ON PERCEPTIONS OF URGENCY WITHIN THE TS}

This section assesses the impact of the planned TS change on perceptions of urgency within the TS in two complimentary ways. First, Section 6.3.5.1 presents findings from an analysis of cross-sectional data. The findings from this stage of the analysis provide us with a view of perceptions of urgency within the TS from the perspective of respondents from all three TS stakeholders. It is within this section that we examine how the planned TS change may have impacted 'sharedness' of perceptions of urgency within the TS. The reader is reminded this data set includes a high number of respondents in the Social Service Agency sample that withdrew from the study. This withdrawal means that, while we are able to make comparisons between TS stakeholders at the group level, we are unable to conduct any analysis at the level of the individual.

Second, in Section 6.3.5.2, we report on the second analysis, which uses panel data. The fact that we are able to compare the responses that a particular individual gave at Time 1 (prechange) to what this person said at Time 2 (post-change) gives us confidence in detecting how the planned TS change may have impacted perceptions of urgency for two groups of stakeholders within the TS post-change.

The final subsection, Section 6.3.5.3, summarizes findings presented throughout this section to address how the planned TS change may have impacted perceptions of urgency over time.

\subsubsection{IMPACT OF PLANNED TS CHANGE ON PERCEPTIONS OF URGENCY:} ANALYSIS OF THE CROSS-SECTIONAL DATA SET 
This section examines how each of the TS stakeholders' views of urgency within the TS may have shifted over time relative to their TS partners' views. We take the following three steps to conduct this analysis.

First, we examined Time 1 data describing how respondents in the three stakeholder groups perceived power within the TS pre-change. As shown in Section 6.3.2, we classified responses (first-order codes and second-order categories) into three categories: (1) common (all three stakeholders perceived a particular action as urgent), (2) shared (two out of three stakeholders perceived a particular action as urgent), and (3) unique (only one stakeholder perceived a particular action as urgent). We also categorized these perceptions as being strong (50\% or more of respondents in each relevant stakeholder group felt an action was urgent) or weak (less than 50\% of the respondents in each relevant stakeholder group felt an action was urgent).

Next, we examined Time 2 data regarding how urgency within the TS was perceived by respondents in the three stakeholder groups six months after the planned change had been implemented. As shown in Section 6.3.4, we classified responses (first-order codes and secondorder categories) into three categories: (1) common, (2) shared, and (3) unique. We also categorized these perceptions as being strong or weak as noted above.

We compared these data and looked for evidence of shifts in 'sharedness' (see Table 57), which we operationally define by comparing the classification we gave a perception of urgency within the TS at Time 1 (e.g. strong common) with the classification we gave the corresponding perception of urgency within the TS at Time 2 (e.g. weak shared). This comparison identifies three different scenarios: (1) no shift in 'sharedness', (2) decreasing 'sharedness' (i.e. more TS stakeholders agreed on urgent actions within the TS at Time 1 than at Time 2), and (3) increasing 
'sharedness' (i.e. fewer TS stakeholders agreed on urgent actions within the TS at Time 1 than at Time 2). In the following section, we present data supporting each of these three scenarios.

No shifts in 'sharedness'. Our analysis suggests that the planned TS change had little impact on how the Social Service Agency was perceived to act urgently within the TS.

- $\quad$ Before and after the introduction of the planned TS change, only respondents from the Social Service Agency reported that they had urgent actions within the TS (weak unique) (i.e. Perceptions of Urgent Social Service Agency Actions).

- Even though community support workers indicated that they acted urgently when they called 911 to request police assistance before any change was introduced into the TS (weak unique), no respondent indicated that this action was urgent after (i.e. Community support worker calls 911 to request police assistance with high risk PMI).

- At Time 1, no respondent felt that community support workers connecting PMIs with community services after Hospital discharge was urgent (i.e. Community support worker connects the PMI with community services after Hospital discharge). After the planned TS change, however, respondents from the Social Service Agency noted that these were urgent actions (weak unique).

The above data support the idea that, even though the planned TS change did not impact the Hospital or Police's view of urgent actions within the Social Service Agency, it did seem to impact how community support workers view urgency within their organization. 


\begin{tabular}{|r|c|c|c|}
\hline Perceptions of Urgency in the TS Over Time & $\begin{array}{c}\text { Pre-Change } \\
\text { Perception (Common } \\
\text { / Shared / Unique) }\end{array}$ & $\begin{array}{c}\text { Post -Change } \\
\text { Perception } \\
\text { (Common / Shared } \\
\text { / Unique) }\end{array}$ & $\begin{array}{c}\text { Shift in } \\
\text { 'Sharedness' }\end{array}$ \\
\hline Perceptions of Urgent Hospital Actions & Weak Unique (H) & Strong Common & Increase \\
\hline Physicians need to assess PMIs in a timely manner & Weak Unique (H) & Strong Common & Increase \\
\hline Physicians discharge PMIs in a timely manner & & Weak Shared (H, SS) & Strong Shared (P, SS) \\
\hline Perceptions of Urgent Police Actions & Strong Common & Weak Shared (H, P) & Decrease \\
\hline Police leave Hospital in a timely manner & Strong Shared (P, SS) & Strong Shared (P, SS) & No Shift \\
\hline Police transport PMI to Hospital in a timely manner & Weak Unique (SS) & Weak Unique (SS) & No Shift \\
\hline Community support worker calls 911 to request police \\
assistance with high risk PMI & Weak Unique (SS) & & Weak Unique (SS) \\
\hline Community support worker connects the PMI with & & \\
\hline
\end{tabular}

Note. Only responses associated with first-order codes that were given a common, shared, or unique label at Time 1 and/or at Time 2 are shown.

Table 57: Perceptions of Urgency within the TS Over Time: Cross-Sectional Data 
Decreasing 'sharedness': Although all three stakeholders mentioned that the Police had urgent actions within the TS before changes were introduced, only two of them felt the Police had urgent actions within the TS after the planned TS change.

- All three TS stakeholders indicated that the Police had urgent actions within the TS before any change occurred (strong common) (i.e. Perceptions of Urgent Police Actions). After the introduction of the planned TS change, however, only the Police and Social Service Agency felt that the Police had urgency within the TS (strong shared).

- In Time 1 interviews, all three stakeholders indicated that the Police leaving the Hospital in a timely manner was an urgent issue for the Police (strong common) (i.e. Police leave Hospital in a timely manner). Post-change, however, fewer respondents in each of the three stakeholders, particularly the Social Service Agency, indicated that Police leaving the Hospital in a timely manner was urgent for the Police (weak shared between Hospital and Police).

These findings suggest that the planned TS change substantially affected how respondents view Police urgency within the TS. More specifically, fewer respondents in each stakeholder indicated that the Police were urgently waiting to leave the Hospital after the planned TS change than before.

Increasing 'sharedness': Only healthcare workers mentioned that the Hospital had urgency within the TS before changes were introduced, however, all stakeholders felt the Hospital had urgency within the TS after the planned TS change.

- Before the introduction of the planned TS change, only the Hospital mentioned that they felt they had urgency within the TS (weak unique) (i.e. Perceptions of Urgent Hospital Actions). Post-change, all three TS stakeholders indicated that the Hospital had urgent actions within the TS (strong common). 
- In Time 1 data, the Hospital mentioned that physicians needing to assess PMIs promptly demonstrated urgency (weak unique) (i.e. Physicians need to assess PMIs in a timely manner). At Time 2, respondents from all three stakeholders noted that physicians were urgently assessing PMIs (strong common).

- In Time 1 data, no respondent mentioned that physicians discharging PMIs promptly was an urgent action (i.e. Physicians discharge PMIs in a timely manner). At Time 2, however, the Hospital and Social Service Agency indicated that this action of discharging PMIs promptly was a form of urgency within the TS (weak shared between Hospital and Social Service Agency).

These findings suggest that, while only the Hospital thought they had urgency at Time 1, all three stakeholders felt the Hospital displayed urgency within the TS at Time 2.

We summarize these findings to the following three points:

- The data suggests that, even though the planned TS change did not impact the extent that the Hospital or the Police observed urgency within the Social Service Agency, it seems to have affected how community support workers think they have urgency within the TS.

- The planned TS change seems to have decreased the extent to which those in the Hospital sample perceive that the Police have urgency within the TS.

- The planned TS change seems to have increased the extent to which those in the all three stakeholders perceive that the Hospital acts urgently within the TS.

To get a better understanding why the 'sharedness' may have shifted in this manner, we turn to our analysis at the individual level in the following section.

\subsubsection{IMPACT OF PLANNED TS CHANGE ON PERCEPTIONS OF URGENCY:}




\section{ANALYSIS OF THE PANEL DATA SET}

Table 58 summarizes changes to perceptions of urgency within the TS over time by using panel data to compare what a respondent mentioned at Time 1 to what that same respondent mentioned at Time 2. Within our analysis of the panel data, we consider a change in perception of urgency within the TS has occurred when a substantive number of respondents gave a response either: (1) at Time 1 and not at Time 2, or (2) at Time 2 and not at Time 1. We arbitrarily define a substantive difference between Time 1 and Time 2 responses as a difference of 5 respondents per TS stakeholder (i.e. 25\% of a TS stakeholder sample).

It should be noted that only those perceptions of urgency that met our criteria for a substantive difference between Time 1 and Time 2 are shown in Table 58. A full analysis of all perceptions of urgency using the panel data set is found in the Appendix.

We conducted a follow-up analysis on each of the perceptions identified as changed over

time. To do so, we examined the comments made by those individuals, and looked for patterns emerging from the data that could explain how the planned TS change may have impacted views of urgency over time. 


\begin{tabular}{|c|c|c|c|}
\hline & $\begin{array}{l}\text { Perception } \\
\text { mentioned }\end{array}$ & $\begin{array}{l}\text { Hospital } \\
(\mathbf{n}=\mathbf{2 0})\end{array}$ & $\begin{array}{c}\text { Police } \\
(\mathbf{n}=\mathbf{2 0})\end{array}$ \\
\hline \multicolumn{4}{|l|}{$\begin{array}{l}\text { Perceptions of Urgent Hospital } \\
\text { Actions }\end{array}$} \\
\hline \multirow{2}{*}{$\begin{array}{l}\text { Physicians need to assess PMIs in } \\
\text { a timely manner }\end{array}$} & $\begin{array}{l}\text { Said T1 } \\
\text { not T2 }\end{array}$ & $\begin{array}{c}3 \\
(15 \%)\end{array}$ & $\begin{array}{c}0 \\
(0 \%)\end{array}$ \\
\hline & $\begin{array}{l}\text { Said T2 } \\
\text { not T1 }\end{array}$ & $\begin{array}{c}10 \\
(50 \%)\end{array}$ & $\begin{array}{c}10 \\
(50 \%)\end{array}$ \\
\hline \multirow{2}{*}{$\begin{array}{l}\text { Physicians discharge PMIs in a } \\
\text { timely manner }\end{array}$} & $\begin{array}{l}\text { Said T1 } \\
\text { not T2 }\end{array}$ & $\begin{array}{c}0 \\
(0 \%)\end{array}$ & $\begin{array}{c}0 \\
(0 \%)\end{array}$ \\
\hline & $\begin{array}{l}\text { Said T2 } \\
\text { not T1 }\end{array}$ & $\begin{array}{c}5 \\
(25 \%)\end{array}$ & $\begin{array}{c}0 \\
(0 \%)\end{array}$ \\
\hline \multicolumn{4}{|l|}{$\begin{array}{l}\text { Perceptions of Urgent Police } \\
\text { Actions }\end{array}$} \\
\hline \multirow{2}{*}{$\begin{array}{r}\text { Police transport PMI to Hospital in } \\
\text { a timely manner }\end{array}$} & $\begin{array}{l}\text { Said T1 } \\
\text { not T2 }\end{array}$ & $\begin{array}{c}5 \\
(25 \%)\end{array}$ & $\begin{array}{c}12 \\
(60 \%)\end{array}$ \\
\hline & $\begin{array}{l}\text { Said T2 } \\
\text { not T1 }\end{array}$ & $\begin{array}{c}1 \\
(5 \%)\end{array}$ & $\begin{array}{c}1 \\
(5 \%)\end{array}$ \\
\hline \multirow{2}{*}{$\begin{array}{l}\text { Police leaving Hospital in a timely } \\
\text { manner }\end{array}$} & $\begin{array}{l}\text { Said T1 } \\
\text { not T2 }\end{array}$ & $\begin{array}{c}16 \\
(80 \%)\end{array}$ & $\begin{array}{c}14 \\
(70 \%)\end{array}$ \\
\hline & $\begin{array}{l}\text { Said T2 } \\
\text { not T1 }\end{array}$ & $\begin{array}{c}0 \\
(0 \%)\end{array}$ & $\begin{array}{c}0 \\
(0 \%)\end{array}$ \\
\hline
\end{tabular}

Note. $\mathrm{T} 1=$ Time $1 . \mathrm{T} 2=$ Time 2 . Shaded cells identify substantive changes in perceptions of urgency.

Table 58: Perceptions of Urgency within the TS Over Time: Representative Panel Data Our analysis of these findings suggest that the planned TS change may have increased the Hospital's urgency within the TS over time. More specifically, half of the Hospital and Police respondents that we interviewed post-change equated urgency with the need for the Hospital to assess PMIs promptly. None of these individuals gave this response during the Time 1 interview. Examination of the views of these 10 healthcare workers and 10 police officers who gave this response reveals that the implementation of the intervention and escalation policy has increased the perceptions of urgency associated with doctors assessing PMIs brought in by Police. It should be noted that, while none of these respondents actually referred to the specific change by name 
(i.e. intervention and escalation policy), they all did speak about the fact that they have observed physicians assess PMIs as soon as they are capable of doing so.

"It's [The intervention and escalation policy is] working because the physicians are seeing that patient quicker so instead of that patient sitting there and being sick and not knowing what's going on they're being assessed. They're being able to be medicated sooner." (ED Nurse)

"I think with the overall process and with the assistance of the hospital and the doctors, they're recognising that there is some time sensitivity when we have all these officers tied up. So they've done their due diligence and they've continued to, not always but for the most part, ensure that they assess the individual as soon as possible." (Police Constable)

Our analysis also suggests that the planned TS change may have increased the Hospital's urgency in a second way. Five of the healthcare workers mentioned that the Hospital had a sense of urgency after the implementation of the planned TS change to discharge PMIs from the Hospital. A follow-up analysis of these five healthcare workers revealed that the MHESU may be displaying more urgent actions than they did prior to the implementation of the planned TS change. There were two rationales provided by the five healthcare workers that suggested the MHESU's urgency increasing. First, some of the respondents suggested that the MHESU might be pressured by the ED staff to psychiatrically assess PMIs and discharge them so that there is more bed space availability for incoming PMIs. One psychiatrist remarked:

"There has been ongoing dialogue between us and the emerge leadership... it is when there are times of surge and overflow of patients that system flaws become most apparent and with our high volumes, surge is becoming the new norm. So we are consistently in dialogue with emerge leadership, and there are changes in process that give us a better handle on 
responding to volumes in the ED and communication of patients that are waiting and our ability to respond to those times of surge from the department of psychiatry... emerge has had the perception that some of the [MHESU] nurses have delayed the acceptance of new patients, correctly or incorrectly, so now we push to free up bed space [in the MHESU] and are more vocal about it."

A second pattern that emerged from the comments of these five healthcare workers was linked to the InSted worker. One MHESU manager described the InSted program in the following way:

"It's an example of a collaborative effort between community agency and Hospital... they have become embedded as part of our team ... accept referrals for patients on site... seamless transition because they are engaging with them [PMIs] right there in MHESU."

Our analysis suggests that the introduction of the InSted program may be linked to increased urgency for the MHESU in that InSted workers are focused on connecting PMIs to services outside of the Hospital. As InSted workers are embedded within the MHESU team, and the fact that they are working towards removing PMIs from the Hospital, our analysis suggests that the efforts of the InSted worker may be increasing urgency within the MHESU to discharge PMIs. This logic is supported by the following comment made by a MHESU manager about InSted workers:

"They are on site so we can refer directly and they can make that direct contact with the patient while they're still there, which seems to speed up their departure. They have the resources to follow up and they have the resources to get case managements, which we don't have. So they are an asset in terms of being able to deal with the patient once the decision is made that they don't need an admission." 
There is also evidence to support the notion that the planned TS change decreased Police urgency within the TS. At least $25 \%$ of the individuals in the Hospital and Police samples noted that, pre-change, the Police urgently acted when they transported PMIs to the Hospital. None of these individuals gave the response post-change. When we dove deeper into the rich data, we found that there were two possible reasons why this might have been occurring. One pattern that emerged from the data was linked to Police thinking that they were getting better at assessing PMIs in the community, and as a result were less worried about these situations of mental health crisis escalating and requiring more Police resources. While it is possible that the Police mental health training has contributed to some of the increased experience and skills in appropriately assessing PMIs, examination of the data reveals that many of the officers think that the more experiences they have at assessing PMIs, the better they get at it.

"I think we're getting better as far as recognizing a lot of these mental health crises and I think that a lot of our officers, because we deal with it so frequently, it's allowing them not to act as a social worker but to better recognize that sort of thing." (Police Constable)

"Our communications are getting better as far as like when we are dispatched to a certain address you query the address and you'll see that we've gotten a number of mental health concerned calls to that address prior so sometimes you can forecast prior just by whether we've been there before and you can kind of get a vibe as far as whether it is going to be a mental health call and in those circumstances." (Police Constable)

Analysis of the healthcare data provided an alternative explanation as to why some respondents changed their view related to the urgency of Police transporting PMIs to the Hospital. Healthcare workers in this group suggested that the introduction of a phone in the ED, one aspect of the intervention and escalation policy, has relieved some of the pressure on Police officers 
before they even arrived at the Hospital. Healthcare workers commented that Police calling the ED allows the ED to prepare in their accommodations of the PMI. It is possible that Police knowing that the Hospital is preparing to take the PMI relieves some of their urgency to arrive at the Hospital in a timely manner so that their wait time clock may start.

"There's a police phone now, so there's a direct line... a dispatcher or whoever, somebody will call us and just give us the heads up. That way we can start working before they even get here." (ED Nurse)

"They phone and then we call the security. So the police know we will be ready for them and they can go." (ED Nurse)

Lastly, findings show that the planned TS change may have decreased Police urgency in a second way. More specifically, nearly all of the healthcare workers and police officers who mentioned the urgency of the Police needing to leave the Hospital and return to their other duties at Time 1, did not mention it at Time 2. Follow-up analysis suggest two reasons why respondents may be changing their view with regards to Police needing to leave the Hospital. First, there is some evidence to suggest that the intervention and escalation policy has impacted the length of time that police officers were waiting for their detained PMI to be assessed by a doctor. Exemplar comments are provided below.

"They don't have to wait in the hospital the hours that they used to wait, which was maybe between four to six hours sometimes. Now they wait two." (ED Nurse)

"We can see there has been a large reduction in wait times for the police." (Hospital Manager)

"The entire process is now expected to happen within two hours." (ED Resource Nurse) 
"...Reducing the wait times for officers and thus decreasing the amount of time before the doctor sees the apprehended party...." (Police Sergeant)

The above comments suggest that, as the length of time police officers are waiting decreases, so does the level of urgency that the Police have.

Second, there was also some evidence suggesting that the increased communication between the Hospital and the Police has reduced some of the uncertainty related to how long police officers would be waiting. Based on the sergeant's comment below, it appears that this interorganizational communication relieves some of the Police urgency by letting sergeants know how long they will be waiting for their resources to become available.

"There are times when we go up there and there are the longer wait times, but the hospital's at least communicating with us a bit more about as to... giving us a reason. Like, they could be short-staffed or they're just buried up there... and at least knowing that, it at least gives us a bit of an explanation as to what's going on. It helps us, especially us supervisors. If we know it's going to be a couple of more hours, then we can redeploy people around and make things work on our end of... it makes things easier for us."

We summarize our analysis of panel data with the following four points:

- The implementation of the intervention and escalation policy appears to have increased the Hospital's urgency in needing to assess PMIs in a timely manner.

- The planned TS change may have increased urgency within the MHESU in two ways. First, the implementation of the intervention and escalation policy may have resulted in ED staff pressuring MHESU staff to accept new PMIs. Second, the introduction of the InSted program may have resulted in InSted workers pressuring MHESU staff to discharge PMIs to the community. 
- The introduction of the direct phone line between the Hospital and Police may have decreased Police urgency in needing to transport the PMI to the Hospital in a timely manner, as Police are now aware that the Hospital is preparing their accommodation before they even arrive.

- The intervention and escalation policy may have decreased Police urgency by reducing the length of time police officers are waiting at the Hospital and by informing sergeants how much longer their resources are unavailable.

\subsubsection{SUMMARY OF HOW THE PLANNED TS CHANGE MAY HAVE IMPACTED PERCEPTIONS OF URGENCY}

In Section 6.3.5, we explored changes to perceptions of urgency within the TS in order to get a better understanding of how the planned TS change may have impacted TS stakeholder urgency. Section 6.3.5.1 and Section 6.3.5.2 suggests that the planned TS change has shifted urgency within the TS from the Police to the Hospital. Our analysis of the interview data suggests that the planned TS change decreased Police urgency, particularly in the eyes of healthcare workers. The implementation of the intervention and escalation policy appears to have resulted in police officers having fewer delays when they bring a PMI into the Hospital. As well, the introduction of the direct phone line has allowed Police to call the Hospital prior to arriving, which may have reduced their urgency to arrive promptly at the Hospital so that the PMI receives care as quickly as possible.

Moreover, the findings from our analysis suggest that the planned TS change has increased urgency for the Hospital. More specifically, it would appear that the intervention and escalation policy has put more pressure on the ED staff to respond to PMIs in a timely manner. This policy may have also indirectly put pressure on the MHESU staff, as ED staff pressure MHESU staff to accept new PMIs. As well, the addition of the InSted worker within the Hospital may have resulted 
in InSted workers pressuring MHESU staff into discharging PMIs to the community in a timely manner.

\subsection{DISCUSSION: IMPACT OF PLANNED TS CHANGE ON PERCEPTIONS OF TS STAKEHOLDER RELATIONSHIPS}

The purpose of this section is to discuss the findings presented throughout this chapter. To remain consistent with the structure of this chapter, the findings will be discussed in the following three subsections: perceptions of power (Section 6.4.1), legitimacy (Section 6.4.2), and urgency (Section 6.4.3) within the TS. It should be noted that, while we are discussing each of these dimensions independently, our analysis would suggest that they interact with each other in an interesting fashion. The interaction between the introduction of the planned TS change and the respondents' perceptions of power, legitimacy, and urgency is discussed in the following chapter (see Chapter 7).

\subsubsection{DISCUSSION: PERCEPTIONS OF POWER WITHIN THE TS}

Section 6.1 explored respondents' perceptions of power within the TS before and after planned changes were implemented in order to get a better understanding of how the planned TS change might have impacted the perceived power dynamics within the TS. Four key findings from that section are first summarized in a bulleted list, and then discussed by drawing from relevant theory below.

- Framing interorganizational relationships within a TS through a power lens is a useful way to view relationships within a TS.

- One TS stakeholder may have more power than that of their TS partner. Our analysis suggests that the Hospital held the most power within the TS prior to the implementation of planned TS change. 
- Perceptions of who has power in a TS can shift over time when a planned change is introduced into the TS. Evidence presented in this section support the notion that power shifted from the Hospital to the Police with the introduction of the planned TS change.

- The perception of who has power and how it is displayed in the TS is in the "eye of the stakeholder".

In the subsections below, we address each of these summary points.

\subsubsection{THE UTILITY OF A 'POWER' LENS}

This research finds that conceptualizing interorganizational relationships through a power lens can be a useful way to view relationships within a TS. Interorganizational power has been theorized in the literature as when Organization A can get Organization B to do something that Organization B would not have otherwise done (Mitchell et al., 1997). While the majority of theoretical development in the area of changes to interorganizational relationships has been limited to the idea that relations may strengthen or weaken over time (Mariotta \& Delbridge, 2012), this research is consistent with stakeholder researchers who argue that power may be used to explain why and how organizations may influence their partners (e.g. Frooman, 1999).

\subsubsection{UNEQUAL DISTRIBUTION OF POWER WITHIN THE TS}

Prior to the implementation of the planned TS change, respondents from all three TS stakeholders were able to give many more examples of how the Hospital displayed power in the TS than how either the Police or the Social Service Agency displayed power in the TS. Theory offers two plausible explanations for this power imbalance. First, Burt (2005) contends that positioning may imply differential advantages for the actors embedded within a TS. For instance, Baum et al. (2012) found that actors benefited from bridging the relationship between two parties. Bridging positioning can provide timely information, resource access, and brokerage opportunities 
(Burt, 2005). Given that the Hospital is uniquely positioned in the PMI process to bridge the PMI's journey from the Police to the Social Service, it is possible that the positioning of the Hospital within the TS may partially explain why the Hospital was perceived to display more power than their partners.

Alternatively, Lewin's conceptualization of gatekeeping could also explain why the Hospital might have been perceived to have more power within the TS than their partners before the introduction of the planned TS change. First, prior to the change, ED physicians (i.e. the Hospital) could be viewed as gatekeepers in the PMI process because they uniquely had the power to decide when to assess the PMIs and, thus, release Police from the Hospital. According to Lewin's (1946a) theorization, gatekeepers (i.e. ED physicians) are very powerful within a system, as they can influence the process of change more than others within the group. This theorization of physicians as gatekeepers within the PMI process may also explain why the decision-makers within the TS developed and implemented a specific change (i.e. the intervention and escalation policy) that was designed to offset some of the decision-making power that ED physicians held within the PMI process.

\subsubsection{THE IMPACT OF PLANNED TS CHANGE ON PERCEPTIONS OF POWER}

Examination of our findings suggest that the implementation of the planned TS change explained in the previous chapter is likely to have affected perceptions of power within the TS over time. Frooman (1999) theorizes that a firm's interorganizational power derives from the resource relationship with their partner, such that a firm has power over their partner when the partner needs critical resources that the firm has. According to Frooman's (1999) conceptualization, if a planned change were to affect the resource relationship between the partners, then theoretically we would expect power may shift from one TS partner to another. Our 
data provides support that such a shift can indeed happen. More specifically, our analysis suggests that the implementation of the intervention and escalation policy ensured that Hospital resources (i.e. physician time) would be shared with Police in a timely manner. This 'enforced' sharing of resources, in turn, seems to have affected the resource relationship between the Hospital and the Police. This may explain why more respondents perceived the Police displaying power and fewer respondents perceived the Hospital displaying power after the planned TS change was implemented within the TS. This finding would also support the idea that one partner's loss of power is associated with another partner's gain as our results indicate that the power shifted from the Hospital to the Police.

\subsubsection{POWER WITHIN THE TS IS IN THE "EYE OF THE STAKEHOLDER"}

Although Frooman's (1999) theory explains how the planned TS change may have impacted who was perceived to have power within the TS, it provides little explanation as to why only certain TS stakeholders perceived power in each of the TS stakeholders. In particular, why were social workers the only respondents to note that the Social Service Agency may display power in the TS? Why didn't Police respondents mention that they displayed power within the TS? And why was the Hospital the only TS stakeholder to have fewer of their staff mention that the Hospital displays power within the TS after changes were implemented?

These three questions are addressed below. First, we draw from previous research in the perception literature that suggests the interpretation of actions within the TS will depend on who is doing the perceiving (Wood, Harms, \& Vazire, 2010). Fundamental attribution error is the tendency for people to place undue emphasis on internal characteristics to explain someone else's behavior in a given situation rather than considering the situation's external factors (Van Dyck, Frese, Baer, \& Sonnentag, 2005). The results presented in this chapter suggests that respondents 
from the Social Service Agency may place undue emphasis on their own organization's power within the TS, as only Social Service Agency respondents mentioned that the Social Service Agency displayed power within the TS.

Interestingly, healthcare workers and police officers did not place any undue emphasis on their own organization's power. Perhaps, this may be due to the fact that the Hospital and Police are a cluster within the larger TS. Patterns of clustering refer to the degree to which the TS is formed of tightly interconnected cliques or clusters (Ahuja et al., 2012). The emergence of interconnected subgroups suggests that the TS is being differentiated into a variety of distinct partnerships. If the Hospital/Police partnership was tighter than that of either the Hospital/Social Service Agency or Police/Social Service Agency partnership, than that might explain why healthcare workers or police officers were less familiar (or aware) with how the Social Service Agency displays power within the TS. This cluster theorization is also supported by literature arguing that cluster instability, which in this case may have been caused by the Hospital making the Police wait for their PMIs to be assessed, may "portend an imminent change in the power structure" (Ahuja et al., 2012, p.437).

Second, to explain why respondents may perceive their TS partner displaying power in ways that were not mentioned by those at the TS stakeholder displaying the power, we draw from the contrast effects literature. A contrast effect is the enhancement (or diminishment) of a perception as a result of successive exposure to a stimulus of lesser or greater value (Ang, Van Dyne, \& Begley, 2003). We suspect that contrast effects may impact respondents' views of how the Police displays power within the TS. While healthcare and social workers are able to contrast the behavior of the PMI without the presence of Police to the behavior of the PMI with the presence of Police, police officers only perceive PMIs in situations where they are present. The fact that 
police officers are successively exposed to the PMI behaving in only a single manner suggests that their perception of the PMI's behavior may be limited. As a result, Police may not be aware of the extent that their presence may influence the flow of the PMI process.

Third, we draw on loss aversion theory from the economics and decision-making literature to contribute to an explanation of why TS stakeholder respondents are more likely to perceive their own losses in power than are respondents representing other TS stakeholders. Loss aversion refers to an individual's tendency to strongly prefer avoiding losses than acquiring gains (Kahneman \& Tversky, 1984). Research in the area suggests that individuals are twice as aware of losses as they are gains (Kahneman \& Tversky, 1992). Our analysis of perceptions of power within the TS preand post-change suggest that the Hospital experienced a loss in power and the Police experienced a gain in power with the introduction of the planned TS change. Loss aversion theory would support the idea that healthcare workers would be more sensitive to their loss in power than Police or Social Service Agency respondents would be to any relative gain in power. Accordingly, loss aversion theory can explain why healthcare workers reported the Hospital lost power over time, even though Police and Social Service Agency respondents did not report this phenomenon.

While our discussion of perceptions of power within the TS has focused on the display of interorganizational power in the context of day-to-day actions, the existing literature suggests that organizations may broadly exert power over their partners. If we were to maintain Mitchell et al.'s (1997) logic towards interorganizational power (i.e. the ability to get others to do something they might not have otherwise done), then we might suspect that the planned TS change itself may be a display of power within the TS if it involved changing organizations in ways that they wouldn't have otherwise changed. Van de Ven and Poole's (1995) dialectic theory argues that, when one organization (or the 'antithesis' using their terminology) gains enough power to overthrow the 
status quo (or the 'thesis' using their terminology), conflict may occur and result in a change (or a 'synthesis' using their terminology). Interestingly, even though the Hospital was perceived to be the TS stakeholder with the most power within the TS before changes were implemented, the data provided within this chapter suggest that the Police changed the Hospital in a way that the Hospital wouldn't have otherwise changed (i.e., Police gained power, Hospital lost power). This counterintuitive finding suggests that interorganizational dynamics may not be explained by examining TS stakeholder power in isolation. To learn more about how Police were able to influence their TS partners in the form of a planned TS change, we turn to our discussion of legitimacy and urgency within the TS.

\subsubsection{DISCUSSION: PERCEPTIONS OF LEGITIMACY WITHIN THE TS}

Section 6.2 explored respondents' perceptions of legitimacy within the TS before and after planned changes were implemented in order to get a better understanding of how the planned TS change might have impacted the perceived legitimacy of TS stakeholders. We begin by articulating the four key findings from that section. This is followed by a discussion of each of these points with the folding in of relevant theory. The following observations of how a planned TS change can impact perceptions of TS legitimacy are supported by our data:

- Framing interorganizational relationships within a TS through a legitimacy lens provides a useful perspective on relationships within a TS.

- One TS stakeholder may have more legitimacy than that of their TS partners. Our analysis suggests that the Police held the most legitimacy within the TS prior to the implementation of planned TS change. 
- Perceptions of who has legitimacy within the TS can shift over time when a planned change is introduced into the TS. Evidence presented in this section support the notion that the legitimacy of the Police eroded in the eyes of healthcare workers over time.

- Respondents can perceive that their own TS stakeholder demonstrates legitimacy in the TS in ways that may not be as obvious to other TS stakeholders.

We address each of these summary points in the subsections below.

\subsubsection{THE UTILITY OF A 'LEGITIMACY' LENS}

This thesis conceptualizes legitimacy as an important indicator of interorganizational relationships. Legitimacy refers to a generalized perception or assumption that the actions of an entity are desirable, proper, or appropriate within some socially constructed system of norms, values, beliefs, or definitions (Suchman, 1995). By conceptualizing interorganizational relationships with respect to perceptions of legitimacy, we are able to draw from the legitimacy literature when exploring how the planned TS change may have impacted TS stakeholder relationships over time.

\subsubsection{PERCEPTIONS OF UNEQUAL LEGITIMACY FOR TS PARTNERS}

Before planned changes were implemented, respondents from all three TS stakeholders were able to give more examples of how the Police displayed legitimacy within the TS than of how either the Hospital or the Social Service Agency displayed legitimacy within the TS. For instance, three quarters of the respondents we interviewed prior to the planned TS change mentioned that police officers demonstrated legitimacy within the TS when they apprehended PMIs under the mental health act. According to Mitchell et al. (1997), managers are more likely to respond to organizations they perceive as legitimate. Theoretically then, we would expect TS stakeholders to respond to the concerns of any TS partner who they deemed to display high 
legitimacy within the TS. This conjecture is supported by the findings from this study. All of the TS stakeholders in our sample perceived the Police demonstrated legitimacy within the TS in many ways and, it would appear from our findings that, Hospital and Social Service Agency management made TS changes to accommodate the Police. This rationale may partially explain why the Hospital would agree to and implement a planned TS change that resulted in them losing power. The linkage between decision-makers' perceptions of legitimacy and the implementation of planned TS change needs, however, greater empirical study in order to better understand this relationship.

\subsubsection{THE IMPACT OF PLANNED TS CHANGE ON PERCEPTIONS OF LEGITIMACY}

This chapter provides some evidence to suggest that the implementation of a planned TS change has the potential to impact perceptions of legitimacy within the TS. For example, approximately a third of Hospital respondents changed their view of the legitimacy in the Police's as well as their own actions within the TS. These changes to views of legitimacy may be partially explained with the existing legitimacy literature below.

First, our results show that the perceived legitimacy of the Police apprehending PMIs under the mental health act declined in the health care sample after the planned TS change was implemented. Just as legitimacy may be bestowed on an organization by external stakeholders who endorse the worthiness of its vision or objectives (Zimmerman \& Zeitz, 2002), an organization's legitimacy may also erode in the eyes of external stakeholders when contradictory objectives emerge in their relationship (Vaara \& Monin, 2010). In this case, it appears that the planned TS change may have impacted how healthcare workers view the Police's objectives in relation to their own. Healthcare workers wanted the Police to improve their mental health training and completion of EDP forms, however, they did not perceive that either of those changes occurred 
(see Section 5.3.1) and feel that police are apprehending PMIs who do not have legitimate reasons for coming to the ED (see Section 6.2.5.2). Accordingly, healthcare respondents may have thought that the Police used the planned TS change to achieve their own objectives without supporting the objectives of the Hospital.

Interestingly, there were specific changes implemented that were designed to improve mental health training for police officers and increase the rate at which EDP forms were completed. Quantitative data also supports the idea that police officers were completing EDP forms more often. Despite the hard evidence supporting the fact that these changes had in fact been implemented, healthcare workers did not perceive that those changes took place. This begs the question: would healthcare workers' views of Police legitimacy still decline if they were aware of the Police's efforts to improve mental health training and completion of EDP forms? Future research may benefit from exploring the relationship between management communicating the TS change initiative, including each organizations' initiatives, and perceptions of legitimacy within the TS over time.

Second, findings show that a subgroup of healthcare workers viewed the Hospital's action of assessing PMIs as legitimate only after the planned TS change was implemented. Drori and Honig (2013, p.347) argue that legitimacy involves "the acceptance or normative validation of an organizational strategy through the consensus of its participants". As an organization's legitimacy may increase through the acceptance of emergent practices (Lounsbury \& Crumley, 2007), then employees accepting an emergent practice (i.e. intervention and escalation policy) may bestow legitimacy upon their own. In this case, the data suggests that, before the planned TS change, a subgroup of healthcare workers did not perceive that they were acting legitimately when they were assessing PMIs because the PMI had to wait a long time before the assessment. Our analysis also 
indicates that, after the introduction of the change, these healthcare workers felt the Hospital was displaying legitimacy when they assessed PMIs because the PMI did not have to wait for a long time before the assessment. These findings would suggest that this subgroup of healthcare workers would have accepted the planned TS change, and in so doing, have increased views of their own legitimacy.

There was also a subgroup of healthcare workers who viewed the Hospital's action of assessing PMIs as legitimate only before the planned changes were implemented. Drori and Honig (2013) argue that an organization's (i.e. Hospital) view of their own legitimacy may erode when exogenous actors (i.e. Police) attempt to bias internal organizational activities toward their own advantage. Our analysis suggests that the subgroup of healthcare workers that viewed the doctor rushing to assess (and sometimes inappropriately forming) PMIs in order to get Police out of the Hospital would think that the Police was biasing the Hospital's activities.

\subsubsection{RESPONDENTS MORE AWARE OF THEIR LEGITIMACY THAN PARTNER'S LEGITIMACY}

It is interesting to note that, although the Social Service Agency participates within the PMI process and was involved in the planning and implementing of the TS change, respondents from neither the Hospital nor the Police perceived the Social Service Agency displaying legitimacy within the TS. This finding is contrary to existing research, which suggests that organizations would only partner with those parties that have legitimate claims that can add value to their own organization (Das \& Teng, 2000). There is one plausible explanation for this finding. If there was a large difference between managers' views of a partner's legitimacy and frontline workers' views of a partner's legitimacy, then we could understand why managers would deem a partnership appropriate while frontline workers would not. Unfortunately, given that we did not conduct the 
same interview with managers as we did with frontline workers, we do not know how differences between manager and frontline workers' views of a partner's legitimacy impact change. Future research may benefit from exploring this issue.

This research also supports existing theory, which argues that individuals may perceive legitimacy in their own actions that may not be seen as legitimate by others outside their organization (Helms \& Patterson, 2014). Existing legitimacy research may partially explain this finding. Drori and Honig (2013) argue that the process of building external legitimacy (i.e. when outsiders view an organization's legitimacy) can have four stages: (1) developing a strong view of your own legitimacy, (2) recognizing the need for external legitimacy, (3) searching for external legitimacy, and (4) initiating external legitimacy. Two findings from this case study suggest that the Social Service Agency is searching for external legitimacy. First, Social Service Agency respondents had a strong unique view of their own legitimacy at Time 1 and Time 2, which would suggest they already view their own organization as legitimate. Second, one of the specific changes that were implemented was starting the InSted program and having a Social Service Agency employee working from within the Hospital. This would suggest that the Social Service Agency is looking for more opportunities to visibly affect the PMI process or that the management of their TS partners do see what they do as legitimate. Unfortunately, our Time 2 data was collected too early for us to see if the Social Service Agency gained external legitimacy from frontline healthcare workers, as a result of healthcare workers interacting with InSted workers within the Hospital. Future research should perhaps use longer time frames between data collection in order to examine Drori and Honig's (2013) process model for developing external legitimacy within a TS undergoing change.

\subsubsection{DISCUSSION: PERCEPTIONS OF URGENCY WITHIN THE TS}


Section 6.3 explored respondents' perceptions of urgency within the TS before and after planned changes were implemented in order to get a better understanding of how the planned TS change might have impacted the perceived urgency within the TS. The following three key findings from that section are summarized and discussed below.

- Framing interorganizational relationships within a TS through an urgency lens provides a useful perspective on relationships within a TS.

- One TS stakeholder may have more urgency than that of their TS partners. Our analysis suggests that the Police displayed the most urgency within the TS prior to the implementation of the planned TS change.

- Perceptions of who has urgency within the TS can shift over time when a planned change is introduced into the TS. Evidence presented in this section supports the notion that urgency decreased for the Police and increased for the Hospital over time.

These summary points are addressed in the following subsections.

\subsubsection{THE UTILITY OF AN 'URGENCY' LENS}

This research finds that considering urgency as a critical dimension of interorganizational relationships can be helpful in understanding how relationships within a TS evolve over time. Urgency refers to the degree to which stakeholder claims call for immediate attention (Mitchell et al., 1997). Urgency is based on the idea that claims are important to the stakeholder and thus delays in attending to the claim are unacceptable to the stakeholder (Mitchell et al., 1997). While there has been very little TS theory (for an exception, see Mariotta \& Delbridge, 2012) explaining the dynamism of interorganizational relationships, our research suggests that including perceptions of TS stakeholder urgency within the TS as a potential contributing factor may increase our understanding of how TS change unfolds over time. 


\subsubsection{PERCEPTIONS OF UNEQUAL URGENCY WITHIN THE TS}

Before the planned TS change was implemented, respondents from all three TS stakeholders were able to give many more examples of urgent actions for the Police than either the Hospital or the Social Service Agency. In particular, almost all respondents mentioned that Police had delays within the Hospital preventing them from returning to their work in the community. Mitchell et al. (1997) would argue that, given that respondents from the Hospital and Social Service Agency perceived the Police as having the most urgency within the TS, healthcare providers and community support workers may have been motivated to change in a way that addressed those perceptions of urgency. This motivation to reduce the Police's urgency may explain why the Hospital agreed to develop and implement the escalation and intervention policy, which was aimed at reducing the amount of time PMIs and police officers had to wait in the Hospital before the PMI was assessed by a doctor. It should be noted, however, that this research did not collect data with respect to perceptions of urgency from decision-makers at each of the three TS stakeholders. As a result, we were unable to confirm whether or not those perceptions of urgency did, in fact, relate to the implementation of the planned TS change. Future empirical research may benefit from making the linkage between decision-makers' perceptions of urgency and the implementation of planned TS change.

\subsubsection{THE IMPACT OF A PLANNED TS CHANGE ON PERCEPTIONS OF URGENCY}

Analysis of the data supports the idea that the implementation of the planned TS change likely changed the urgency, and in turn perceptions of urgency, within the TS over time. The extant literature offers two explanations for why urgency in the TS has shifted over time. First, 
Mitchell et al. (1997) theorize that stakeholders have urgency when their partner delays in attending to their claims. The theoretical idea of a partner delaying in attending to important claims may be associated with police officers waiting a long time in the Hospital for a physician to assess their detained PMI. If the Police's urgency primarily stems from police officers waiting in the Hospital, then the implementation of a specific change that was designed to decrease the amount of time police officers had to wait (i.e. intervention and escalation policy) may decrease the Police's urgency. As we showed with quantitative data in Section 5.2.4, we have confidence that the intervention and escalation policy did substantially decrease Police wait times. Accordingly, this change may explain why fewer respondents mentioned how the Police were urgently waiting to be released from the Hospital after the planned changes were implemented than before.

Second, Rowley and Moldoveanu (2003) would argue that a planned TS change may also increase the feeling of discontent within a particular group, and that this discontent may generate this group's urgency to act. Data suggests numerous reasons why healthcare workers may feel discontent from the planned TS change. First, as noted previously, healthcare workers wanted the Police to improve their mental health training and their completion of EDP forms at Time 1 but did not observe those changes at Time 2. Second, police officers are apprehending and bringing to the Hospital more PMIs whom healthcare workers do not feel have legitimate mental health concerns, which unnecessarily consumes the Hospital's resources. Third, findings support the idea that healthcare workers have experienced added pressures, particularly from the Police, to assess and discharge PMIs promptly. Fourth, our analysis of quantitative data would suggest that, while healthcare workers are assessing PMIs in a timelier manner after the planned TS change has been implemented than before, they are making other patients (whom they may feel have more legitimate issues) wait longer. Thus, according to Rowley and Moldoveanu (2003), given that the 
planned TS change has increased healthcare workers' discontent within the TS, it is also likely to have increased their urgency within the TS.

Interestingly, while there were no changes to perceptions of urgent Social Service Agency actions over time, one of the four specific changes (i.e. the InSted program) was designed to impact the timeliness of connecting PMIs from the Hospital to appropriate community services. Existing theory provides some explanation why perceptions of urgency did not change after the implementation of the planned TS change. On the one hand, the implementation of the InSted program meant that social workers would respond more quickly to PMIs discharged from the Hospital. These time sensitive activities in responding to PMIs should have, according to Mitchell et al. (1997), resulted in community support workers having more urgency within the TS. On the other hand, introduction of the InSted program may have contented community support workers, such that they did not have to locate PMIs after they were discharged from the Hospital (a concern of theirs noted in Section 6.2.1). Thus the content gained from the InSted program should have, according to Rowley and Moldoveanu (2003), decreased urgency within the Social Service Agency.

The above noted rationales, complimented with the finding that perceptions of Social Service Urgency did not change over time, suggest that perceived urgency is two-dimensional. The first dimension, as theorized by Mitchell et al. (1997), is related to the perceived time sensitivity of an action. In other words, perceived urgency is likely to increase when individuals have less time to perform a task. The second dimension, as theorized by Rowley and Moldoveanu (2003), is associated with the idea that individuals may be motivated to act based on their discontent with a particular partnership. In other words, perceived urgency is likely to increase when individuals become more discontented with a partnership. This two-dimensional 
conceptualization of urgency might explain why there were no changes to perceptions of Social Service Urgency. In particular, any increase in urgency associated with time sensitivity may have been counteracted with a decrease in urgency concerning the content of the change. Future research should explore this two-dimensional conceptualization of urgency further, and how increasing one dimension and decreasing another dimension of urgency may impact the TS and TS change. 


\section{CHAPTER 7: CONCLUSIONS}

This exploratory case study responds to calls for greater attention to questions associated with how change unfolds in TSs over time (Ahuja et al., 2012; Gulati, 1998) and the role of interorganizational relationships within the TS change process (Kim et al., 2006). It has been the premise of this research that a stakeholder framework (Mitchell et al., 1997) is suited to capturing the central elements of an interorganizational relationship and thus provides the conceptual tool needed to better understand interorganizational relationships within a change context. Although the majority of research in the change management domain focuses on change within organizations (Burnes \& Cooke, 2012; Armenakis et al., 1999), this research draws from the change, TS (i.e., strategic alliance, interorganizational network, cross-sector collaboration), and stakeholder literatures to investigate how a planned TS change may impact perceptions of TS stakeholder relationships over time.

This final chapter is divided into four sections. We first address how the findings from this research inform our research objective regarding 'sharedness' (Section 7.1). We discuss our findings concerning 'sharedness' first to set the stage for summarizing and discussing our findings associated with our primary research objective in Section 7.2. Section 7.3 discusses the contributions of this research, while the final section (Section 7.4) acknowledges limitations and identifies directions for future research.

\subsection{THE UTILITY OF THE 'SHAREDNESS' CONCEPT FOR STUDYING PLANNED TS}

\section{CHANGE}

'Sharedness' is the degree to which cognitions, preferences, identities, or perceptions are shared within groups (Tindale \& Kameda, 2000). 'Sharedness' is grounded in two fundamental ideas: (1) groups of individuals can be conceptualized as information-processing systems, and (2) 
the degree to which perceptions are shared and are being shared within the group affect group dynamics (Tindale \& Kameda, 2000). While 'sharedness' has been traditionally viewed as a lens for analyzing group performance and group decision-making (Kerr et al., 2004), this research deviates from much of the existing 'sharedness' literature by applying the 'sharedness' concept to individuals working across organizational boundaries within a TS. Our analysis suggests that, while 'what is perceived' may be important, 'who shares those perceptions' is also very important to understanding change within a TS.

This conceptualization of the 'sharedness' concept within a TS change context has allowed us to understand the dynamics of what is happening within a TS undergoing change in three unique ways. First, this conceptualization of 'sharedness' has allowed us to explore who within the TS has shared perceptions (i.e., common, shared, unique). Second, we can also examine the strength of those shared perceptions (i.e., weak, strong). Third, this conceptualization facilitates an analysis of the stability of those shared perceptions under conditions of planned change (i.e. do those perceptions change over time).

We illustrate these three aspects of 'sharedness' within the following model (see Figure 9). Each circle in the Venn diagram represents analysis of the data given by the respondents representing each TS stakeholder in our TS: Hospital, Police, and Social Service Agency. The diagram is drawn so that the extent to which the three circles overlap represents the degree to which the views of employees from the three TS stakeholders were found to exhibit 'sharedness': common, shared, or unique. Responses that were operationalized as common perceptions are listed in the middle region of the diagram where all three circles overlap (e.g. First-order code 2 at Time 1). Responses that were operationalized as unique perceptions are shown in the nonoverlapped region of the circle representing the TS stakeholder with the unique view (e.g. First- 
order code 1 and First-order code 4 at Time 1). Responses that were operationalized as shared perceptions are shown in the overlapped region between the circles of the TS stakeholders sharing this perspective (e.g., First-order code 3 and First-order code 5 at Time 1). Second-order categories are included in capitals, while first-order categories are included in lower case. We show strong and weak perceptions in the model in the following manner: perceptions classified as strong are bolded (e.g. First-order code 3), while perceptions classified as unique in shared situations are not (e.g. First-order code 5). The model also includes our analysis of data pre-change (i.e. Time 1) and post-change (i.e. Time 2). A comparison of pre-change and post-change Venn diagrams allow us to easily identify how 'sharedness' has shifted in the TS in response to the planned TS change (e.g. First-order code 1 shifted from a weak unique perception to a strong shared perception).

It should be noted that this type of pictorial display of key findings is also used in our refined conceptual model, which is presented and discussed in more detail in the following section (see Section 7.2).

Our analysis of the 'sharedness' data presented throughout Chapters 5 and 6 suggests that 'sharedness' is very important to our understanding of interorganizational relationships within a TS. One of the major conclusions of this thesis is that a planned TS change can impact 'sharedness' across organizational boundaries over time. This conclusion is based off three major findings regarding 'sharedness' within the context of a planned TS change. Our analysis found that in response to the introduction of a planned TS change: (1) who shares perceptions can shift over time, (2) how strongly the perceptions are shared can shift over time, and (3) what is shared (e.g., power, legitimacy, urgency) can also shift over time. 
TIME 1

TIME 2

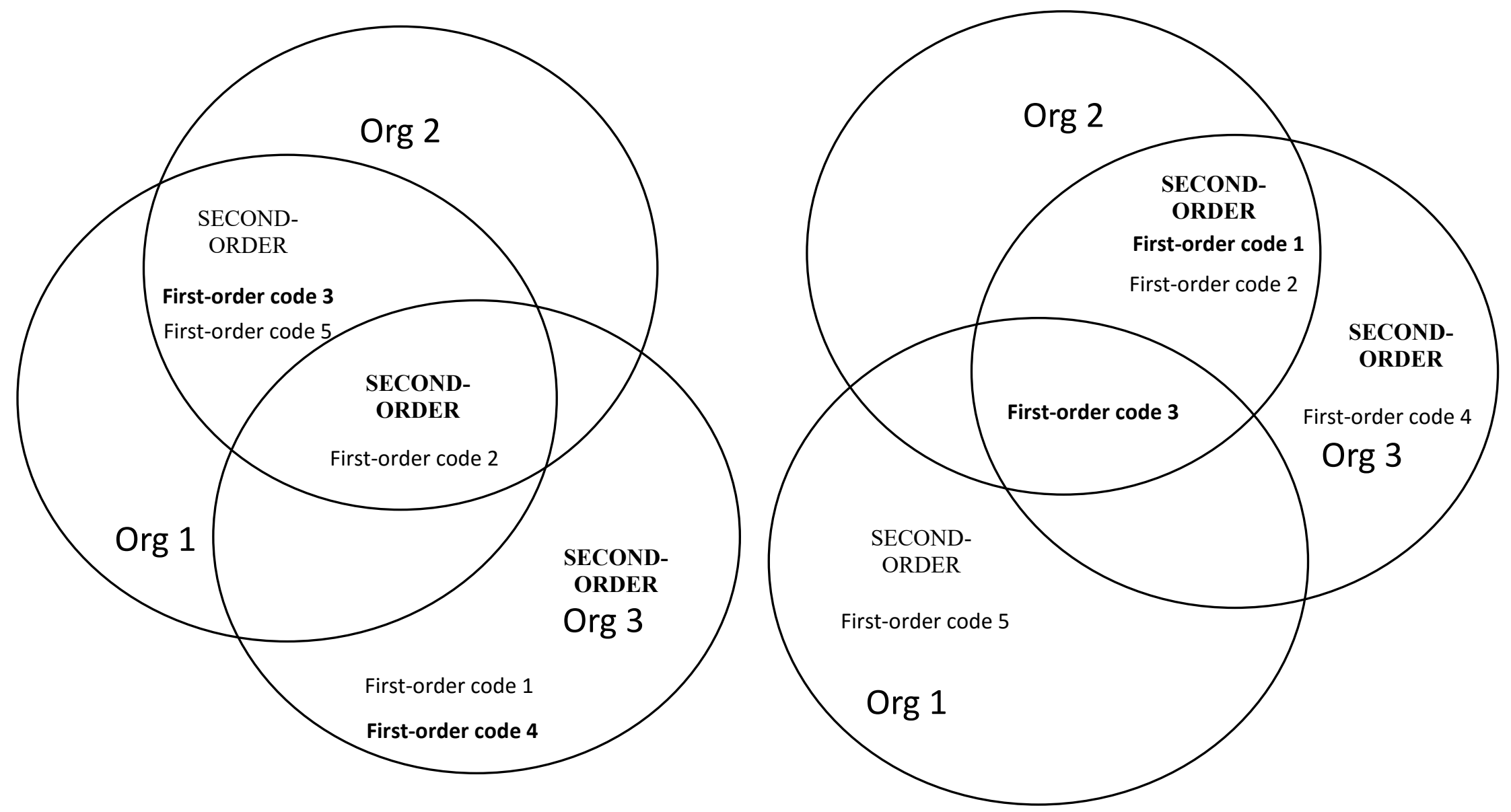

Figure 9: Example of Venn diagram used to summarize key findings regarding 'sharedness' 
These conclusions have implications for researchers and practitioners. From a research perspective, this thesis emphasizes how TS change may be more complex than that of organizational change by showing that a planned TS change can impact 'sharedness' between TS stakeholders within a TS. As 'sharedness' has been shown to impact group dynamics (Tindale \& Kameda, 2000), it is also likely to impact how change unfolds within a TS over time. Future researchers may benefit from using this conceptualization and operationalization of 'sharedness' when examining higher levels of change to get a deeper understanding of the interorganizational dynamics.

It is also important for managers to know that, while they may hold their own perceptions within the TS, other stakeholders may have similar or different views of the situation. For example, managers may think that their organization has power within the TS, while their partners may not have the same perception. In this example, recognition of such a perceptual difference is important, as organizations may be motivated to behave in different manners based on those perceptions. As well, managers should be aware that a planned TS change can shift 'sharedness' within the TS. It is recommended that managers use planned TS changes as an opportunity to communicate across organizational boundaries and provide sensegiving to their partners, which would shape the way that their partners make sense of the TS.

\subsection{DEVELOP A FRAMEWORK DESCRIBING HOW A PLANNED TS CHANGE IMPACTS PERCEPTIONS OF TS STAKEHOLDER RELATIONSHIPS OVER TIME}

The primary objective of this research was to develop a framework describing how a planned TS change impacts perceptions of TS stakeholder relationships over time. Our preliminary conceptual framework explaining how a planned TS change might impact perceptions 
of TS stakeholder relationships over time was developed in Chapter 3 and, for the convenience of the reader, is shown (again) in Figure 10.

Time 1

Perceptions of TS

Stakeholder Relationships

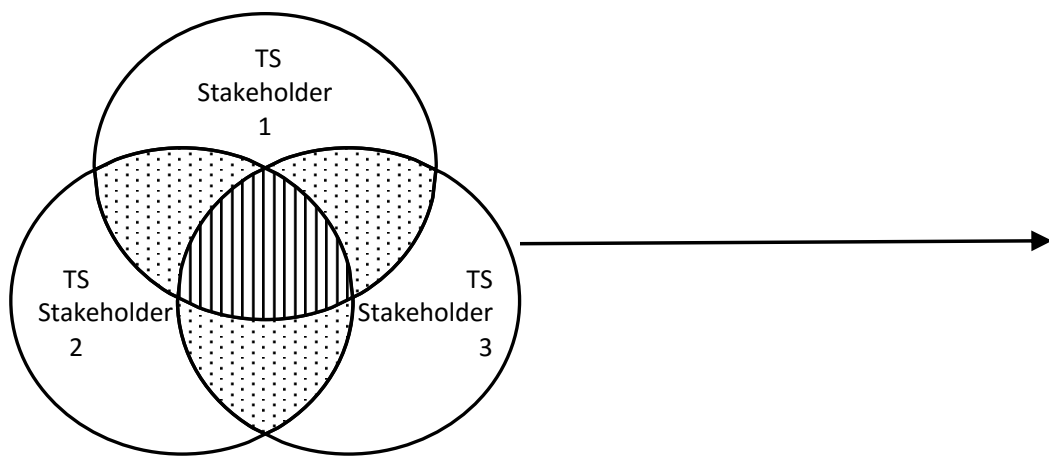

Time 2

Perceptions of TS Stakeholder Relationships

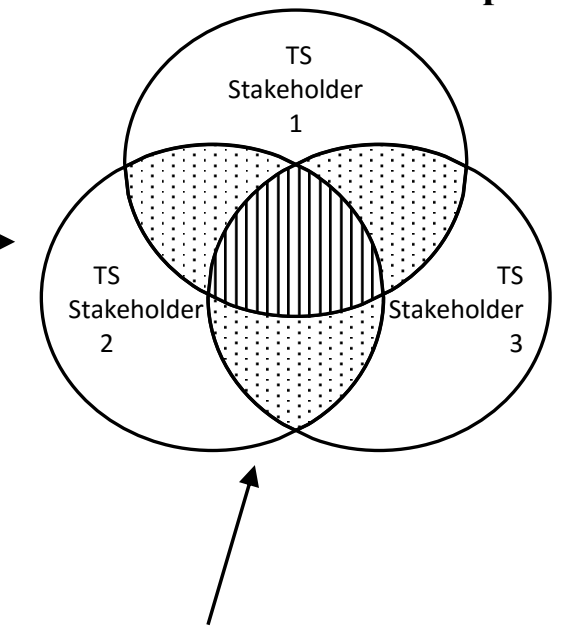

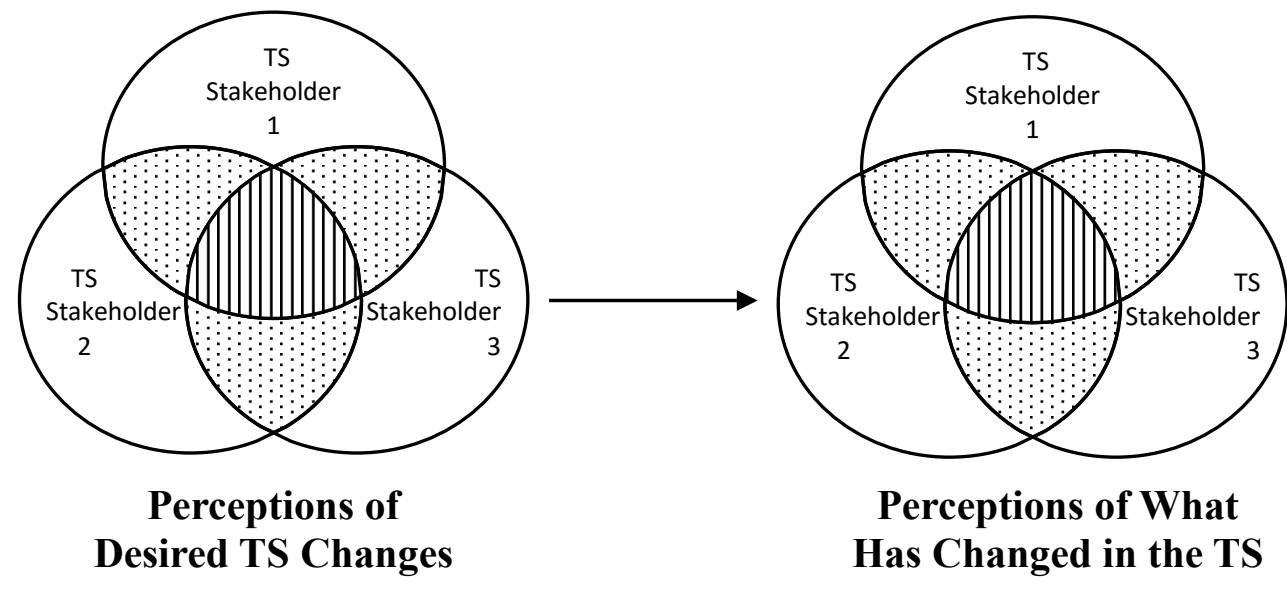

Figure 10: Revisiting the preliminary conceptual framework

Our initial conceptualization of how a planned TS change may impact perceptions of TS stakeholder relationships had four constructs and three paths connecting them. The first two constructs, Perceptions of TS Stakeholder Relationships at Time 1 and at Time 2, referred to each 
TS stakeholder's perceptions of the extent to which they and the other TS stakeholders in the TS possesses power, legitimacy, and urgency within the TS before (i.e. Time 1) and after (i.e. Time 2) the introduction of the change, respectively. The next two constructs related to the change. More specifically, Perceptions of Desired TS Changes referred to what each TS stakeholder perceived needed to change within the TS, while Perceptions of What has Changed in the TS referred to each TS stakeholder's perceptions of what had changed in the TS after the introduction of planned change to the TS.

Within this initial conceptualization, we theorized that perceptions of TS stakeholder relationships at Time 1 impacted perceptions of TS stakeholder relationships at Time 2. This path was supported by research that suggested interorganizational relationships within a TS tend to be sustained over time (Peteraf, 1993) and that institutional arrangements and cultural orientations that characterize these business systems are usually very stable (Bachmann, 2003).

Our initial model also hypothesized that perceptions of desired TS changes would impact perceptions of what changed in the TS over time. This theoretical linkage was supported by researchers who have conceptualized the change process by modeling step-wise linkages between 'planning desired changes' and 'consolidating and institutionalizing the new state' (e.g., Armenakis \& Bedeian, 1999; Galpin, 1996).

The final linkage in our preliminary framework conceptualized perceptions of what has changed in the TS affecting perceptions of TS stakeholder relationships at Time 2. This theoretical path was supported in the literature by researchers who have argued that changes to the resources within a TS may impact power (Frooman, 1999), legitimacy (Lounsbury \& Glynn, 2001), and urgency (Rowley \& Moldoveanu, 2003). 
The findings of the research reported in Chapter 5 and Chapter 6 now enable refinement and, where indicated, expansion of the preliminary conceptual framework. More specifically, analysis of the various data collected as part of this case study determined that, while some of the constructs and paths within our preliminary framework were supported by our results, others were not. Accordingly, we used the findings from this case study to develop a new framework describing how a planned TS change may impact perceptions of TS stakeholder relationships over time. This framework is shown in Figure 11 and described below.

It should be noted that four colors have been added to the framework to facilitate comparisons between the Time 1 (pre-change) and Time 2 (post-change) data. Sections shaded in red refer to second-order categories concerning the Hospital (e.g. Perceived Hospital Power). Portions shaded in blue reflect second-order categories regarding the Police (e.g. Perceptions of Legitimate Police Actions). Regions shaded in green show second-order categories involving the Social Service Agency (e.g. Perceptions of Urgent Social Service Actions). Lastly, sections shaded in grey refer to second-order categories regarding system level changes (e.g. Increased Interorganizational Communication). These display conventions allow the reader to easily identify how the planned TS change impacted 'sharedness' with respect to perceptions of both TS relationships and change by comparing the situation pre-change to that of post-change (e.g. compare which sections were shaded blue, green, red, and gray pre-change to which regions were shaded blue, green, red, and gray post-change). 


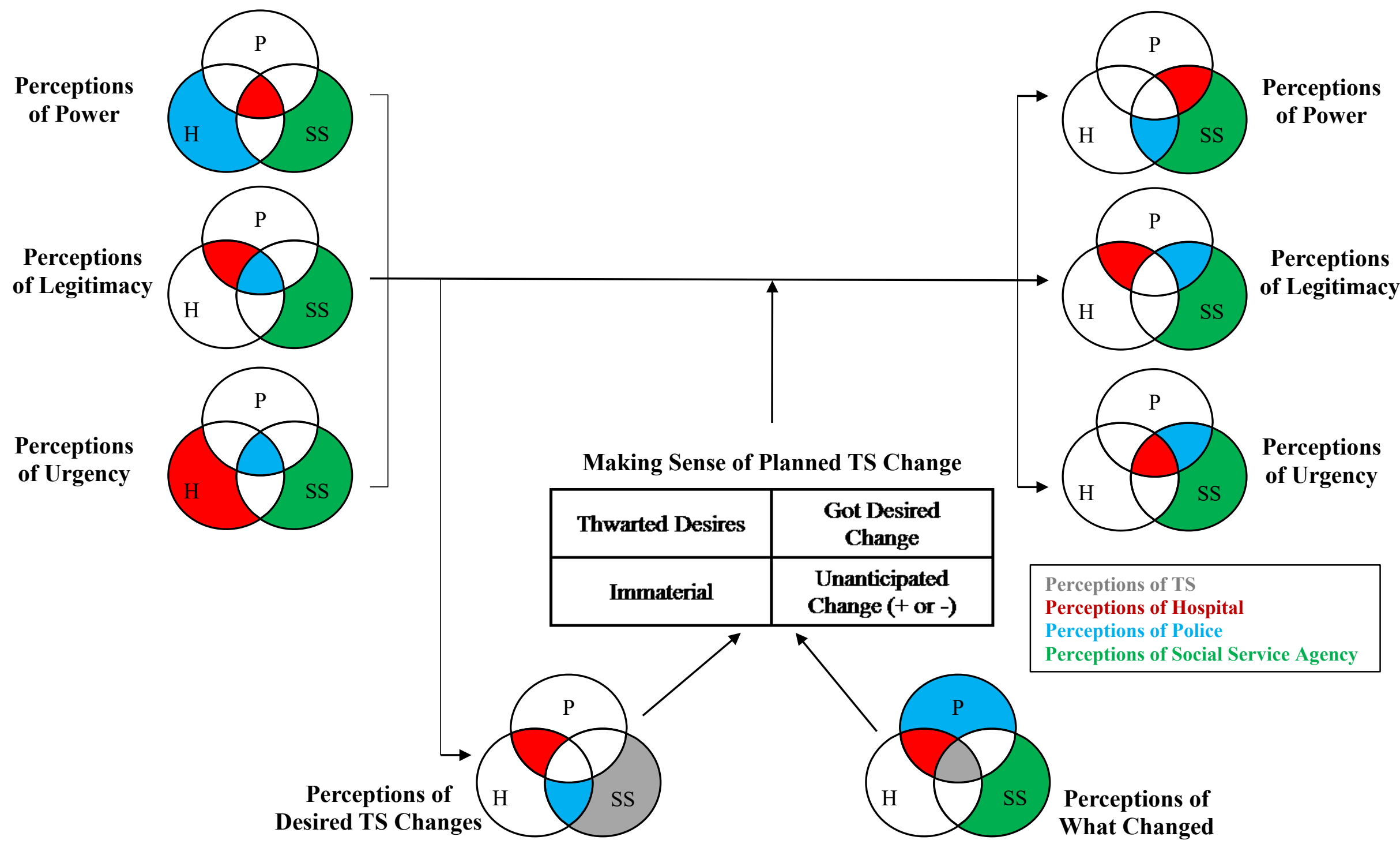

Note: $\mathrm{H}=$ Hospital respondents' perceptions; $\mathrm{P}$ = Police respondents' perceptions; SS = Social Service Agency respondents' perceptions. Only second-order categories are shown in the framework. Areas shaded in gray represent perceptions at the system level. Areas shaded in red represent perceptions of the Hospital. Areas shaded in blue represent perceptions of the Police. Areas shaded in green represent perceptions of the Social Service Agency.

Figure 11: Refined Theoretical Framework of how planned TS change impacts TS stakeholder relationships over time 
This section of the conclusion is divided into four parts. We first discuss each of the constructs in our model. In particular, we summarize findings associated with perceptions of TS stakeholder relationships at Time 1 and at Time 2 (see Section 7.2.1), perceptions of desired TS changes and perceptions of what has changed in the TS (see Section 7.2.2), and the typology of making sense of planned TS change (see Section 7.2.3). After each of the constructs are explained, we discuss the paths between the constructs in Section 7.2.4.

\subsubsection{PERCEPTIONS OF TS STAKEHOLDER RELATIONSHIPS}

In developing this part of the model, we were guided by the following research questions:

How do TS stakeholders' perceptions of (a) power, (b) legitimacy, and (c) urgency within the TS before the implementation of the planned TS change compare to their perceptions of (a) power, (b) legitimacy, and (c) urgency within the TS six months after the implementation of the planned TS change?

The orienting framework conceptualized TS stakeholder relationships using Mitchell et al.'s (1997) three dimensions of power, legitimacy, and urgency. This conceptualization was used to model TS stakeholder relationships before the implementation of the planned TS change, as well as post introduction of the change. Within this section, we use Venn Diagrams to summarize key findings associated with perceptions of TS stakeholder power (Figure 12), legitimacy (Figure 13), and urgency (Figure 14) to help answer each of the three research questions.

Perceptions of Power at Time 1 (Figure 12) draws on the data from Chapter 6 to show how power was displayed within the TS before the implementation of the planned TS change. Our analysis indicates that, before changes were implemented, there was a strong common view that one of the TS stakeholders (i.e. Hospital) held all of the power within the TS. This unequal distribution of power is consistent with the fact that two of the TS stakeholders were dependent on 
them sharing their resources (i.e. physicians assessing PMIs) within the TS (Frooman, 1999). Other evidence presented in Chapter 6 shows that respondents from a second TS stakeholder (i.e. Police) felt that they had no power within the TS, even though respondents from the powerful stakeholder (the Hospital) thought their TS partner displayed some power when they impacted another group's behavior (Mitchell et al., 1997). Finally, while respondents from the third TS stakeholder (i.e. Social Service Agency) also reported that they had power within the TS, their TS partners did not share this view. In other words, while there was some agreement within the TS as to which partner had power over the system prior to the change, there were other areas of disagreement.

Follow-up analysis using the Perceptions of Power at Time 2 data supports the idea that the planned TS change had a considerable impact on respondents' perceptions of how power was displayed within the TS. More specifically, the findings indicate that respondents from the powerful stakeholder (i.e. Hospital) thought that they lost power within the TS post-change, even though respondents from the other two stakeholders still shared the perception that they still had all the power. Also noteworthy are the findings showing that respondents from the TS stakeholder who felt powerless pre-change (i.e. Police) didn't think they gained any power within the TS post change. This is interesting as respondents from both of their TS partners shared the notion that they did. These Time 2 findings suggest that the planned TS change impacted the perceived power dynamics within the TS.

Based on the above analysis, we conclude that a planned TS change may shift perceptions of power within the TS from the stakeholder who was perceived to have the most power prechange (i.e. Hospital) to one of their partners (i.e. Police). 
PERCEPTIONS OF POWER AT TIME 1

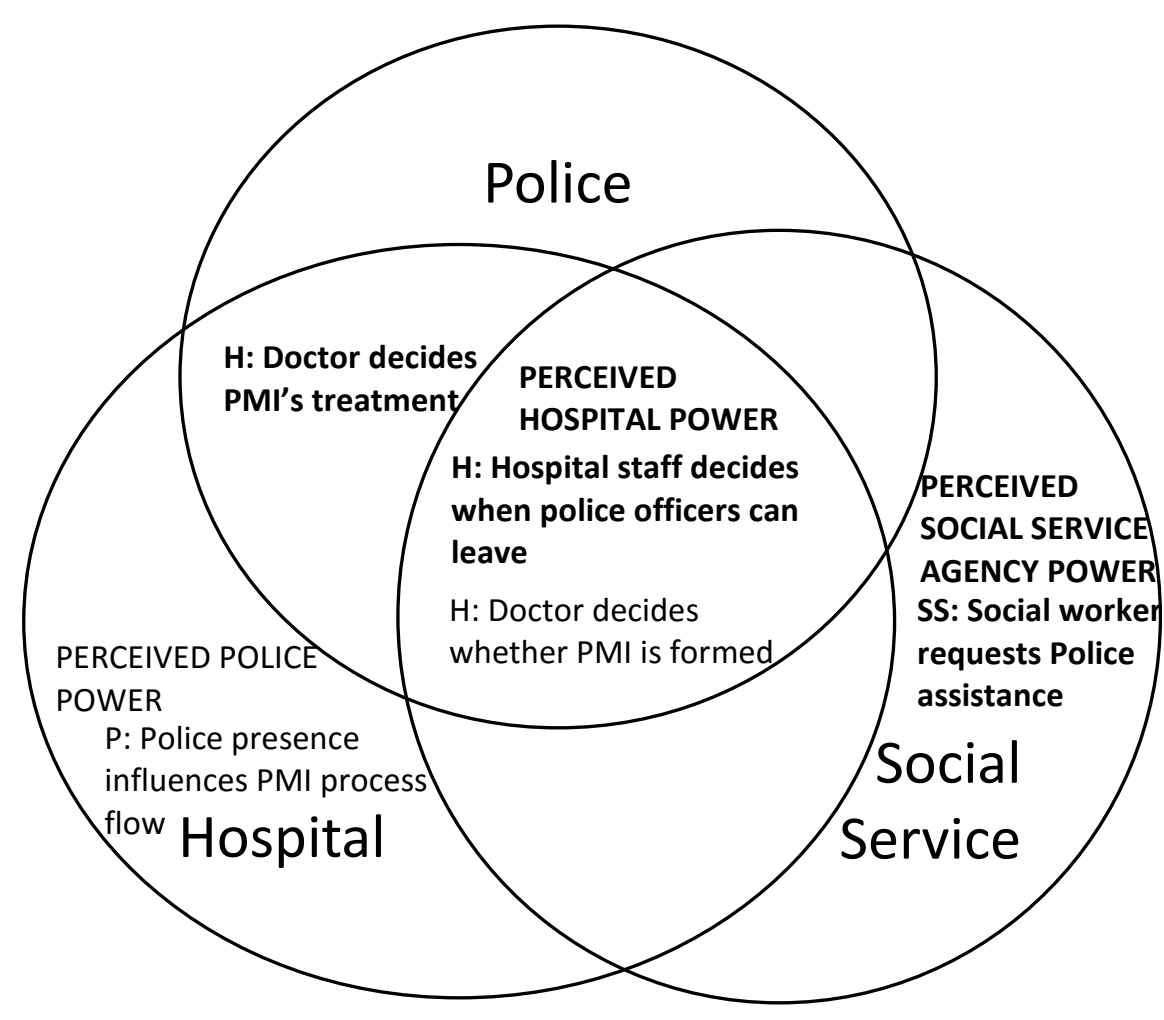

PERCEPTIONS OF POWER AT TIME 2

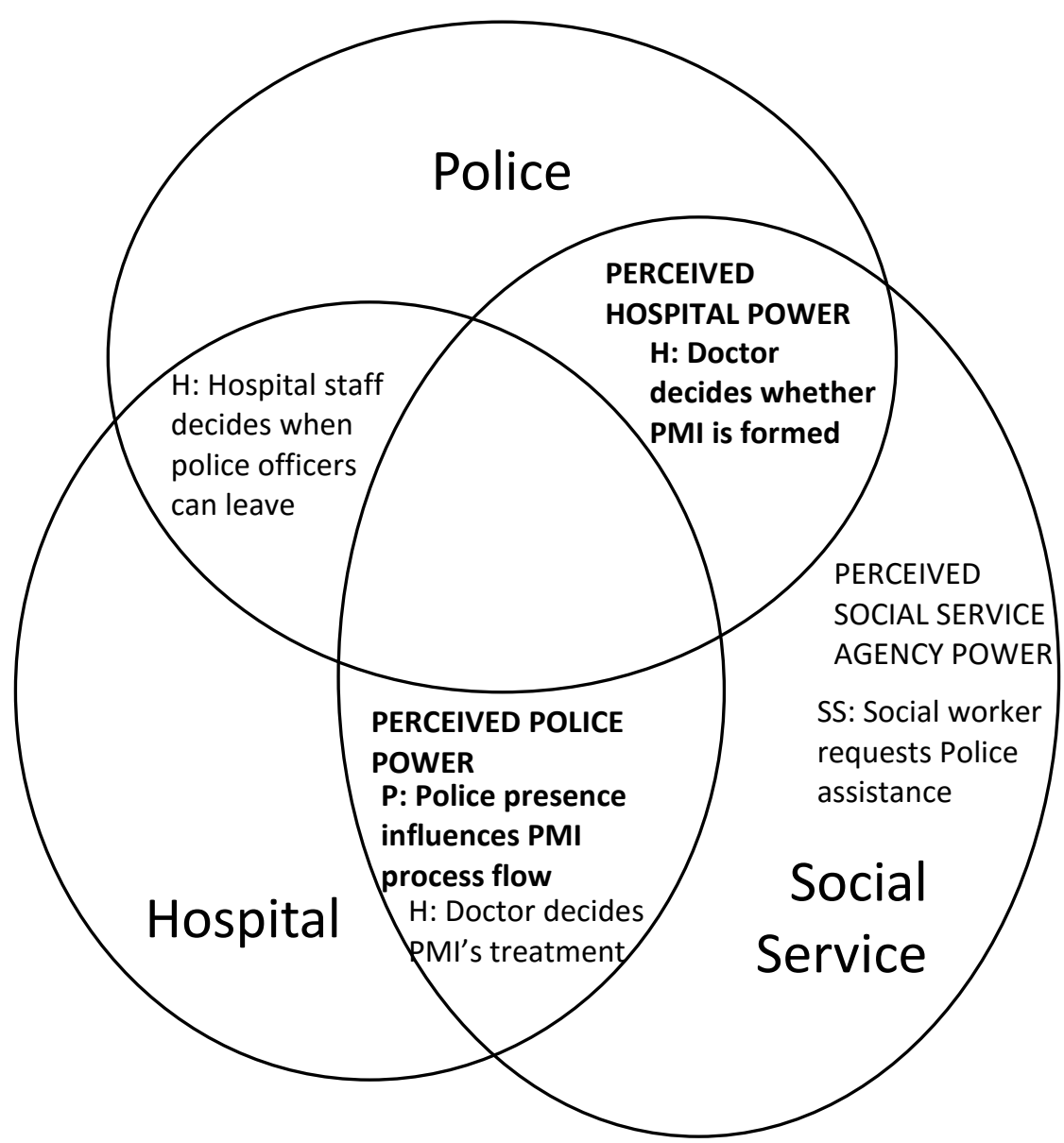

Note: $\mathrm{H}=$ Perceived Power Displayed by Hospital; $\mathrm{P}=$ Perceived Power Displayed by Police; SS = Perceived Power Displayed by Social Service Agency. Second-order categories are capitalized. Strong perceptions are bolded.

Figure 12: Summary of Perceptions of Power within the TS: Pre- and Post-Change 
Perceptions of Legitimacy at Time 1 (Figure 13) draws on the data from Chapter 6 to show how legitimacy was demonstrated within the TS before the implementation of the planned TS change. Our analysis suggests that there was a strong common view that one of the stakeholders held the most legitimacy within the TS (i.e. Police), as their actions within the TS were legitimized by the government (i.e. apprehending PMIs under the mental health act) (Suchman, 1995). Our findings support the notion that the powerful stakeholder (i.e. Hospital) was also seen to have legitimacy within the TS, as without them sharing their resources (i.e. expertise in assessing and treating PMIs), the TS would not function. Respondents from the third stakeholder (i.e. Social Service Agency) reported that they had legitimacy within the TS, although this was not reported by others within the TS.

Perceptions of Legitimacy at Time 2 summarizes data presented in Chapter 6 associated with how legitimacy was displayed within the TS six-months post-change. Findings suggest that the planned TS change had little impact on legitimacy within the TS. Respondents from the stakeholder perceived to display the least power within the TS (i.e. Social Service Agency) remained as the only group of respondents that thought that the organization that they worked for had legitimacy within the TS. While respondents from the legitimate stakeholder (i.e. Police) continued to share the perception that they played a legitimate role in the TS (i.e. apprehending PMIs under mental health act), it appears that the planned TS change impacted how this role was viewed by respondents from the powerful stakeholder. The perceived legitimacy of the powerful stakeholder (i.e. Hospital) remained relatively constant over time, despite the introduction of the planned TS change, as their two TS partners continued to share the perception that they play a legitimate role within the TS (i.e. assessing and treating PMIs). 


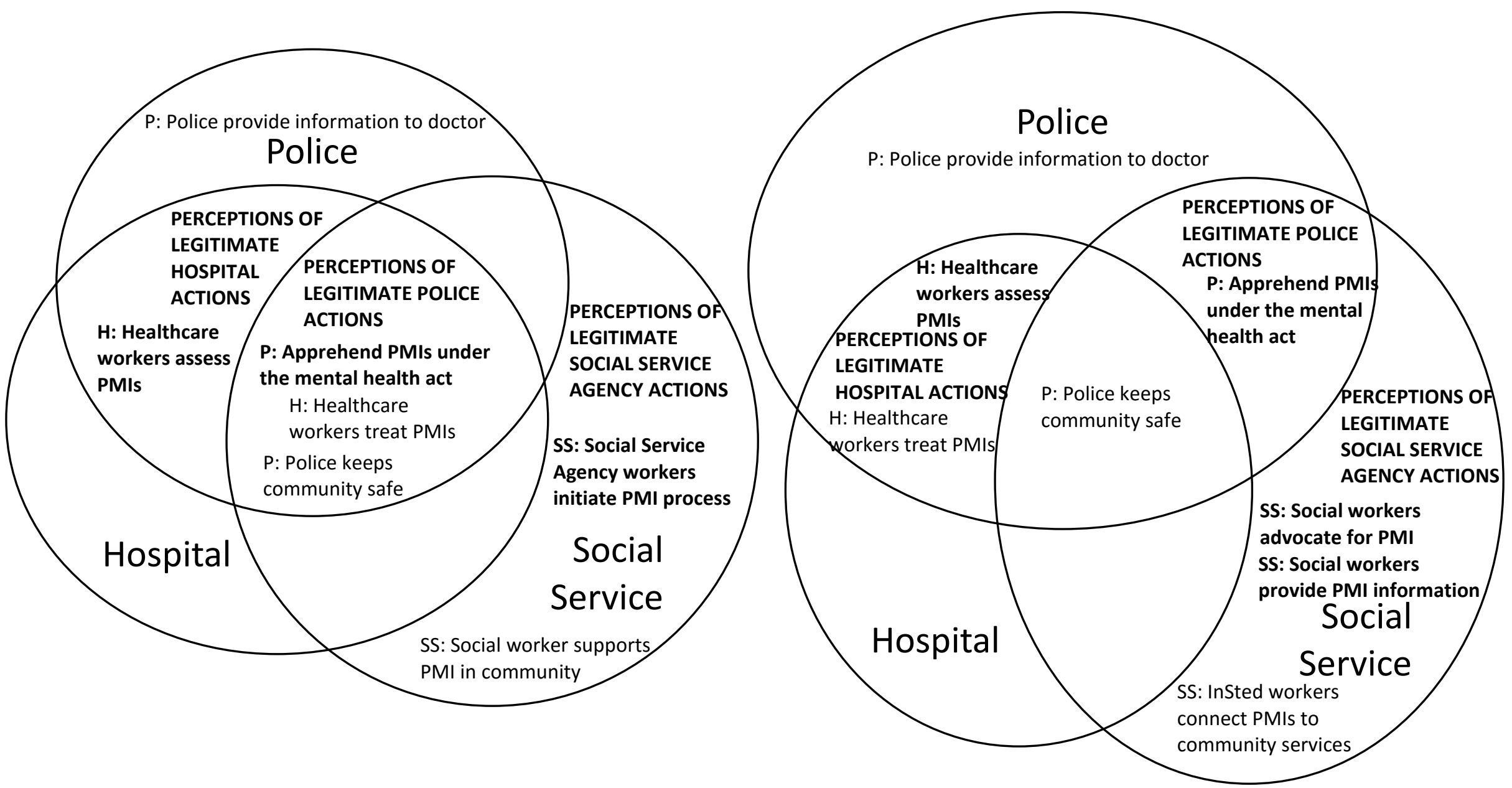

Note: $\mathrm{H}=$ Perceived Hospital Legitimacy; P = Perceived Police Legitimacy; SS = Perceived Social Service Agency Legitimacy. Second-order categories are capitalized. Strong perceptions are bolded.

Figure 13: Summary of Perceptions of Legitimacy within the TS: Pre- and Post-Change 
In summary, our analysis of the Time 2 findings indicates that the planned TS change did not impact the perceived legitimacy of all three TS stakeholders equally, as the 'sharedness' of one TS stakeholder's legitimacy decreased over time.

The above analysis supports the following two conclusions: (1) a planned TS change may impact perceptions of legitimacy within a TS such that a partner who is perceived to have high legitimacy may lose some of their legitimacy (in the eyes of their TS partners) as a result of the change, and (2) a planned TS change may impact employees' views of how their TS stakeholder (e.g., Hospital, Social Service Agency) acts legitimately within the TS.

Perceptions of Urgency at Time 1 (Figure 14) summarizes what actions were considered urgent within the TS before the implementation of planned TS change. Our analysis suggests that there was a strong common view that the legitimate stakeholder (i.e. Police) also held the most urgency within the TS, as one of their partners (i.e. Hospital) delayed responding to them (i.e. Police leaving Hospital in a timely manner) (Mitchell et al., 1997). Examination of the data presented in Chapter 6 also suggests that respondents from both the powerful (i.e. Hospital) and powerless (i.e. Social Service Agency) stakeholder thought that their organization acted urgently within the TS, however, employees outside their organizations did not share these views. 
PERCEPTIONS OF URGENCY AT TIME 1

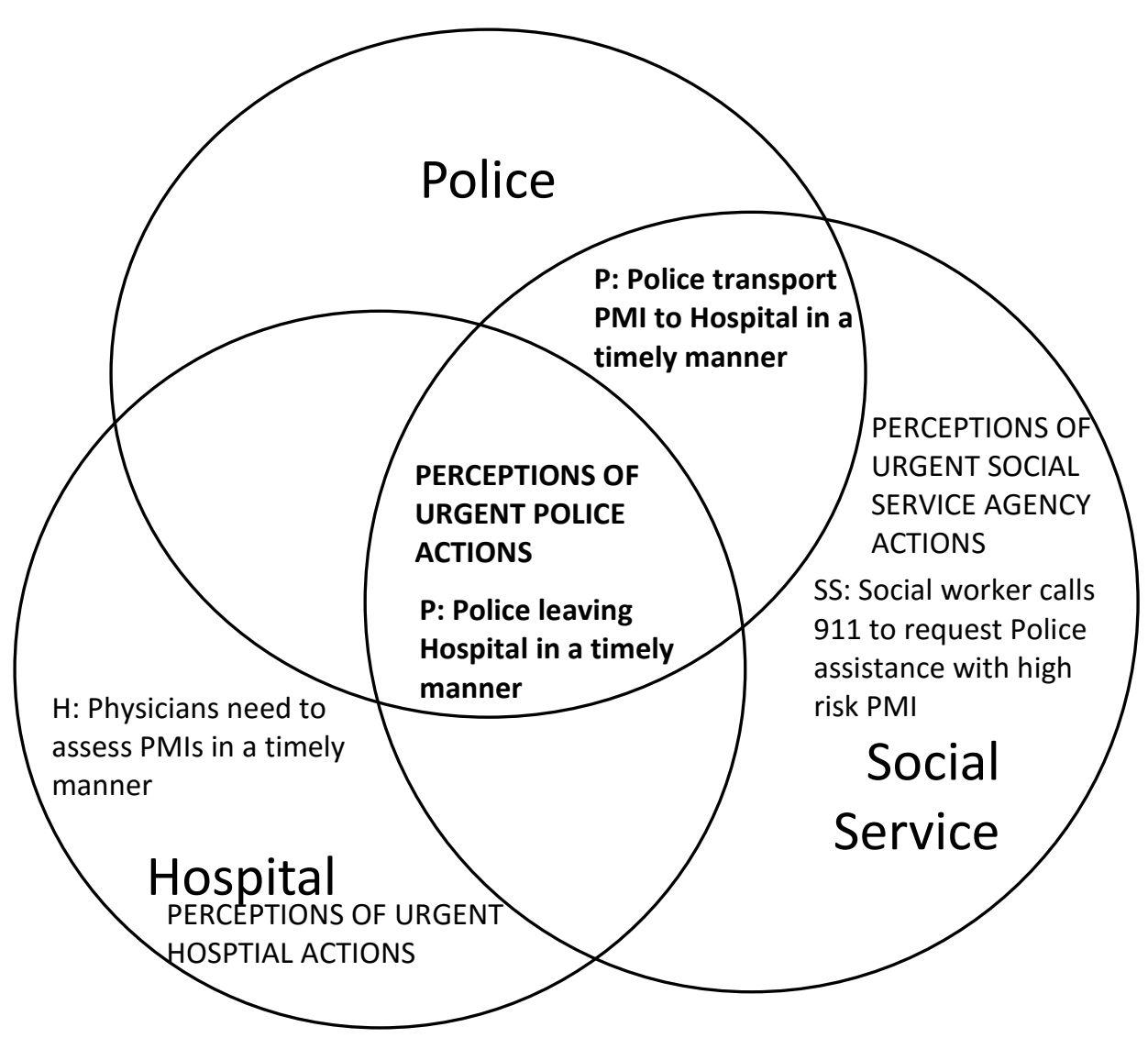

PERCEPTIONS OF URGENCY AT TIME 2

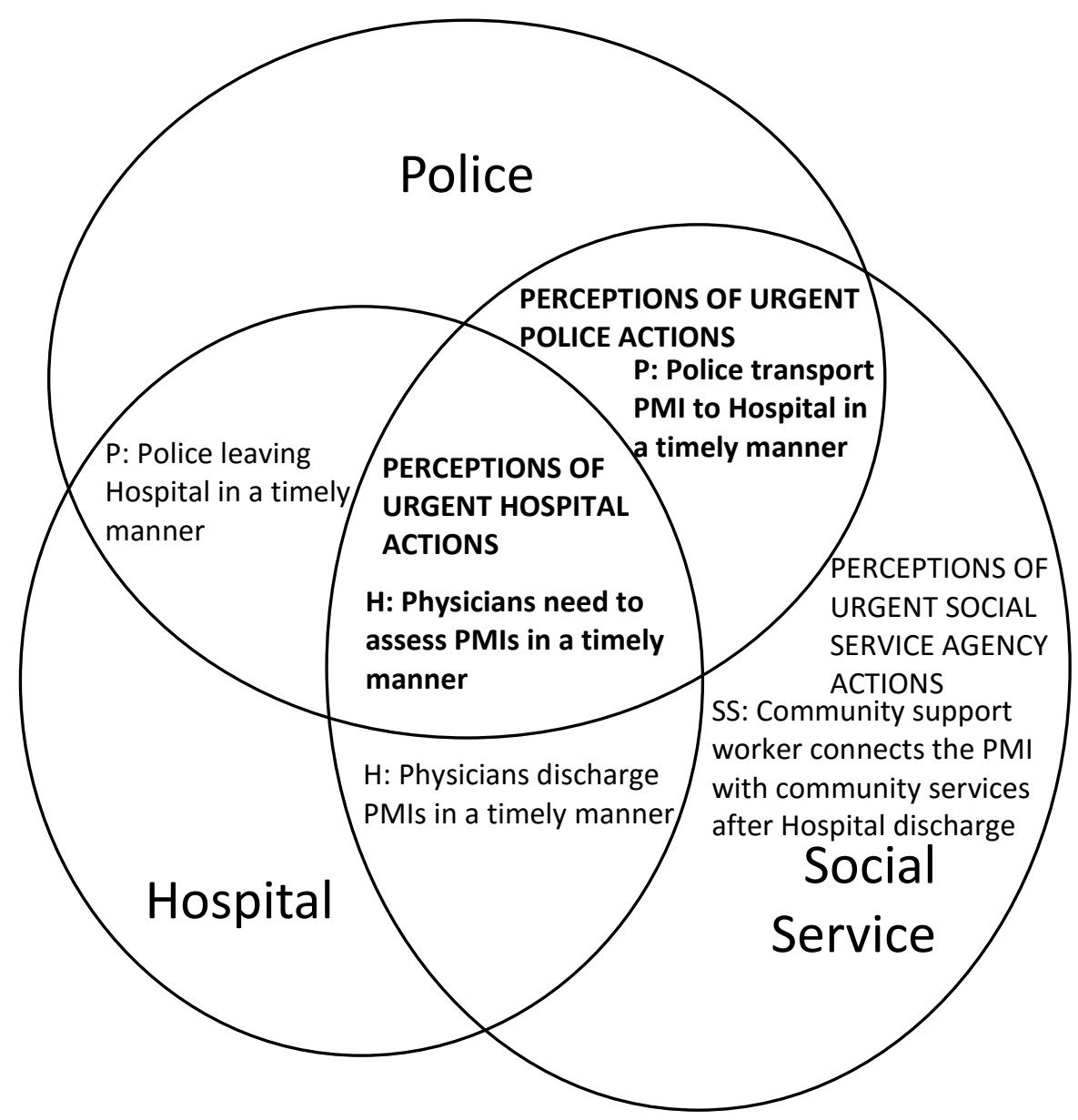

Note: $\mathrm{H}=$ Perceptions of Urgent Hospital Actions; $\mathrm{P}=$ Perceptions of Urgent Police Actions; SS = Perceptions of Urgent Social Service Agency Actions. Second-order categories are capitalized. Strong perceptions are bolded.

Figure 14: Summary of Perceptions of Urgency within the TS: Pre- and Post-Change 
Data associated with actions that were considered urgent within the TS six months after the implementation of the planned changes are summarized in Perceptions of Urgency at Time 2. Analysis of the data collected for this thesis suggest that the introduction of the planned TS change had a considerable impact on who was seen to have urgency within the TS. First, the data supports the idea that the planned TS change has led to an increased sense of urgency within the TS for the powerful stakeholder (i.e. Hospital), as there was a high degree of 'sharedness' between all three TS stakeholders that they have begun responding to the legitimate stakeholder (i.e. Police) promptly. It would seem that the powerful stakeholder reducing the delays to the legitimate stakeholder also reduced the sense of urgency for the legitimate stakeholder. Respondents from the powerless stakeholder (i.e. Social Service Agency) continue to be the only group of respondents who think that they have urgency within the TS. Taken as a whole, our analysis provides evidence to support the idea that the planned TS change increased urgency within one TS stakeholder and decreased urgency within another.

Two conclusions may be drawn from the above analysis: (1) a planned TS change may shift perceptions of urgency within the TS from the stakeholder who was perceived to have the most urgency pre-change (i.e. Police) to one of their partners (i.e. Hospital), and (2) a planned TS change may impact employees' view of how their TS stakeholder (e.g. Social Service Agency) acts urgently within the TS.

Combining these three sets of findings, we can say that one of the major conclusions of this thesis is that a planned TS change can shift perceptions of power, legitimacy, and urgency within a TS. This conclusion has notable implications for change, TS, and stakeholder researchers, as well as practitioners. 
This research has implications for Lewin's field theory in that it may provide some insight into how drivers of change may become barriers to change (and vice versa) in response to a planned TS change. For example, analysis of our perceived legitimacy data showed that healthcare workers' views of Police legitimacy (a driver of change) decreased as a result of the change. Such shifts in perceptions could potentially create barriers to future collaborations within the TS. Future research may benefit from exploring perceptions of drivers and barriers of change within the TS, as well as how a planned TS change may transition drivers to barriers of change (and vice versa).

These findings also have implications for TS theorists. While researchers have traditionally viewed changes to interorganizational relationships from the perspective of either strengthening or weakening relationships (Mariotta \& Delbridge, 2012; Gulati, 1998); this research finds that changes to interorganizational relationships are much more complex than previously conceptualized. For example, this research finds a planned TS change can (1) impact perceptions of interorganizational power, legitimacy, and urgency within the TS, (2) impact 'sharedness' of those perceptions, and (3) differentially impact each TS stakeholder (e.g. impacts Hospital power but not Social Service Agency power). Future research may benefit from further exploration into how interorganizational relationships change over time. One way that researchers may do this is by exploring how other possible dimensions of interorganizational relationships change over time. Bachmann (2001; 2003), for example, argues that trust is a critical dimension of interorganizational relationships. Future research may benefit from exploring how a planned TS change impacts interorganizational trust over time.

These results have implications for stakeholder theory, which has largely been viewed from a static perspective (Key, 1999). This research supports the idea that a planned TS change can make stakeholders more or less powerful, legitimate, or urgent over time. These results are also 
important to Mitchell et al.'s (1997) theory of stakeholder salience. Findings suggest that it is not only important what stakeholders are perceived to have power, legitimacy, and urgency, but also who shares those perceptions. In this thesis, we found the views that were commonly shared by all parties were most influential within the TS, and also most likely to shift as a result of the planned TS change. We recommend that future stakeholder researchers follow our lead and explore the 'sharedness' of perceptions of power, legitimacy, and urgency within the TS when considering stakeholder salience.

These conclusions are also relevant for practitioners in three main ways. First, managers should keep in mind that a planned TS change may shift perceptions of power between two parties within a TS. This finding is important for both those who have power within the TS and those who do not. On the one hand, managers who are employed at TS stakeholders who are perceived to have power within the TS should keep in mind that, while a joint change initiative may allow them to provide a new service to their customers (in this case PMIs), it also may result in them losing some of their power to one of their TS partners. On the other hand, managers who are employed at TS stakeholders who are not perceived to have power within the TS might see a joint change initiative as an opportunity to gain more power within the TS.

Second, practitioners need to be wary that a planned TS change may negatively impact perceptions of legitimacy within a TS. This thesis provides evidence to suggest that, while one of the TS stakeholders (i.e. Police) was largely benefiting from the change, the legitimacy of this stakeholder was reduced in the eyes of one of their key TS partners (i.e. Hospital). Managers employed at TS stakeholders who are benefiting from a planned TS change should be cautious of the optics associated with only themselves benefiting from the change. 
Lastly, it is important for managers to note that a planned TS change may shift perceptions of urgency from one partner to another within a TS. Similar to that of power, this finding is critical for managers employed at TS stakeholders who either have urgency or do not. In situations where a TS stakeholder is not perceived to have urgency within the TS, managers should be wary of entering into planned TS changes as they may gain more urgency within the TS. In situations where a TS stakeholder is perceived to have urgency within the TS, managers are encouraged to enter planned TS changes as they may reduce their urgency within the TS.

\subsubsection{PERCEPTIONS OF DESIRED TS CHANGE AND PERCEPTIONS OF WHAT HAS CHANGED IN THE TS}

The following research question guided the development of the Perceptions of Desired TS Change and Perceptions of What Has Changed in the TS constructs within the refined framework: "How do TS stakeholders' perceptions of what needs to be changed within the TS compare to their perceptions of what has changed within the TS six months after the implementation of the planned TS change?"

To support our analysis of perceptions of desired TS changes and perceptions of what has changed in the TS, we analyzed the change from four perspectives. We first looked at the four changes that were agreed upon by decision-makers to get an indication of intended changes to the TS. Second, we examined data associated with four objective indicators of change to get an understanding of how the system actually changed over time. Third, we examined respondents' perceptions of desired TS changes to get an understanding of what they wanted to change. Lastly, we explored what respondents observed had changed within the TS to get an understanding of what changes the respondents actually felt had taken place. This combination of perspectives has allowed us to triangulate our analysis and develop a rigorous framework that reflects what our data 
told us about desired versus actual change. A high level summary of the findings associated with each of these perspectives is provided below.

Four specific changes that make up the planned TS change. There were four specific changes agreed upon by decision-makers and implemented within the TS. First, the Police modified their mental health training program. This specific change reflects a TS stakeholder developing their own resources (i.e. skills to assess PMIs in community) so that they are less dependent on their TS partner's resources (Das \& Teng, 2000). Second, the In Short Term Emergency Deviation (InSted) Program was created to support the knowledge transfer between the Hospital and the Social Service Agency. This type of change, which was designed to facilitate knowledge transfer between partners, has been commonly cited within the TS literature (e.g., Mesquita, Anand, \& Brush, 2008). Third, the Police modified their existing protocol for completing Emotionally Disturbed Person (EDP) forms. As the purpose of this specific change was to ensure that Police officers were filling out the EDP form to give healthcare practitioners more background information on the PMI, this specific change reflects a higher quality of information transferred between partners (Mesquita et al., 2008). The final change involved the creation of a new ED process that expedites service for PMIs and Police. This specific change represents a change to the duration of interaction between TS partners (Kale, Singh, \& Perlmutter, 2000).

Analysis of objective indicators of change. Our analysis of the quantitative data presented in Chapter 5 shows that the planned TS change has impacted the TS in three primary ways. First, data analysis shows that the percentage of PMIs apprehended by Police and brought to the Hospital has increased from Time 1 to Time 2. This finding suggests that the frequency of interactions between partners has increased as a result of the planned TS change. Second, data 
analysis indicates that there have been increases over time to both the percentage of EDP forms completed by Police and the percentage of PMIs apprehended by Police and formed by a physician. These findings suggest that the planned TS change has increased the knowledge transfer between TS stakeholders. Third, examination of the data shows the average length of time PMIs apprehended by Police have to wait to be assessed by a physician has decreased over time. This finding supports the idea that the planned TS change has decreased the length of time that TS stakeholders interact.

Respondents' perceptions of desired TS changes. Based on the interview data presented in Chapter 5, Perceptions of Desired TS Changes (see Figure 15) shows changes that frontline respondents wanted to see in the TS as part of the planned TS change. Findings suggested that, as there was little 'sharedness' between TS stakeholders as to what changes respondents felt were necessary within the TS, each TS stakeholder likely had readiness to change in different ways (Armenakis et al., 1999). Evidence presented in Chapter 5 supports the notion that the legitimate stakeholder (i.e. Police) wanted the powerful stakeholder (i.e. Hospital) to reduce delays in responding to them (i.e. reduce wait times for officers who bring in PMIs under the mental health act). Our analysis also suggests that the powerful stakeholder (i.e. Hospital) perceived that they needed to make changes to reduce the urgency of their TS partner. Findings also show that the powerful stakeholder (i.e. Hospital) also wanted to improve the knowledge transfer between the two TS stakeholders (i.e. fill out EDP forms on a regular basis), a finding consistently found within multi-organization change initiatives (Mesquita \& Lazzarini, 2008; Inkpen, 2000; Uzzi \& Gillespie, 2002). Respondents from the powerless stakeholder (i.e. Social Service Agency) reported that they wanted more communication between TS stakeholders and more training for the legitimate stakeholder (i.e. improve mental health training for police officers). In summary, 
respondents from each of the TS stakeholders wanted their partners to change instead of themselves, and thus had a vested interest in collaborating across boundaries so that they could mutually benefit from joint initiative (Rowley \& Moldoveanu, 2003).

Finally, it is also noteworthy that three of the four changes that were implemented by decision-makers were also changes that were mentioned by frontline workers as ones they would like to see implemented. The creation of the InSted program, the only exception, was not mentioned by any respondent as a change they wanted to see in the planned TS change. 


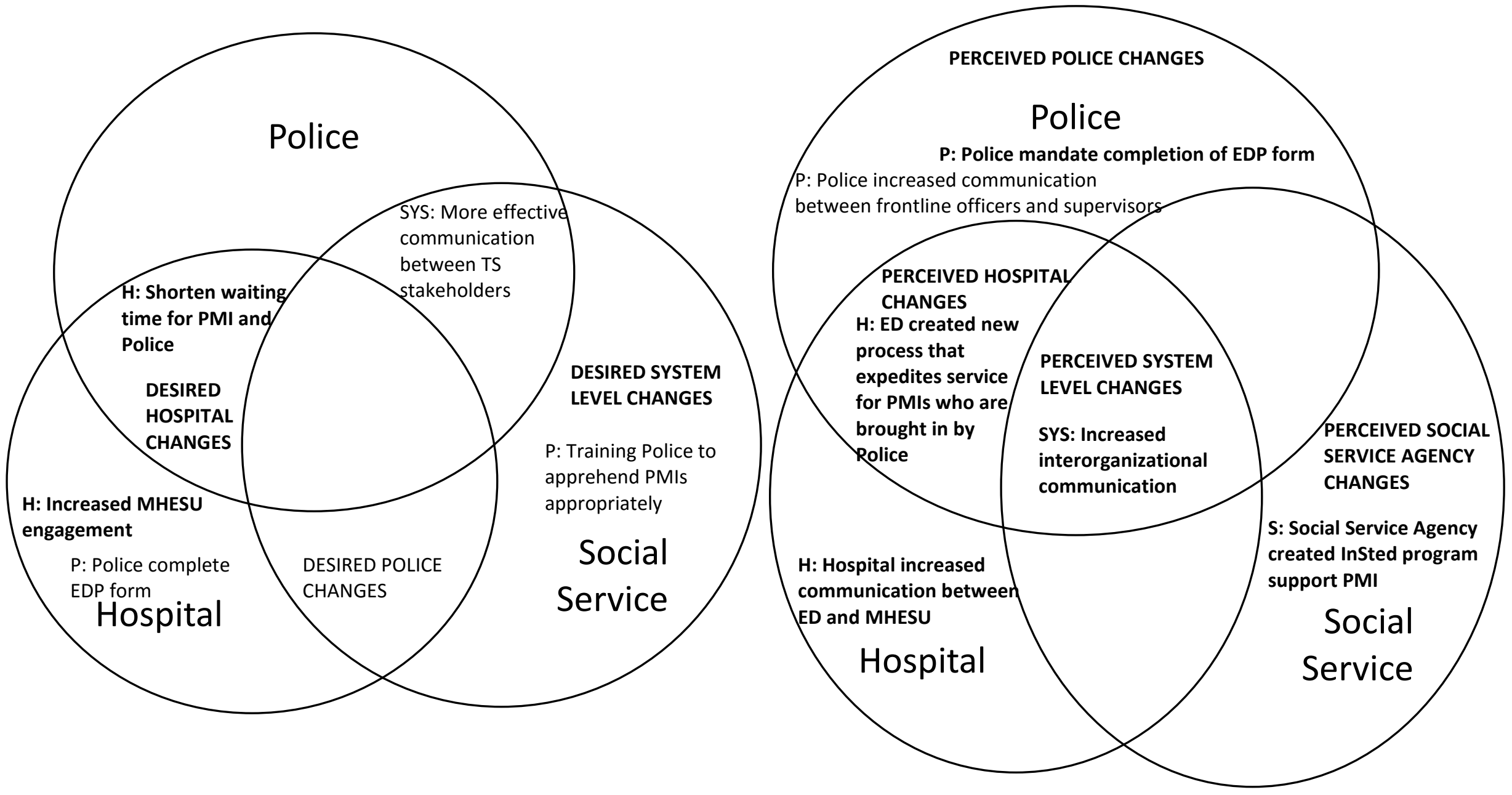

Note: $\mathrm{H}=$ Desired/Perceived Hospital Changes; $\mathrm{P}=$ Desired/Perceived Police Changes; SYS = Desired/Perceived Changes at System Level; SS = Desired/Perceived Social Service Agency Changes. Second-order categories are capitalized. Strong perceptions are bolded.

Figure 15: Summary of Perceptions of Desired and Perceived Changes within the TS: Pre- and Post-Change 


\section{Respondents' perceptions of what has changed in the TS. Perceptions of What Has}

Changed in the TS summarizes findings associated with what respondents observed had changed within the TS six months after the implementation of the planned changes. Our findings show that, while there was a strong common view that the communication had increased between TS stakeholders (a perceived change that was not directly linked to any of the four specific changes introduced to the TS), there were several unique views as to what respondents observed changing within individual organizations. Respondents from the TS stakeholder that was perceived to display the most power (i.e. Hospital) reported observing organizational changes associated with their own behavior (i.e. a process that expedites service for PMIs) and communication (i.e. increased MHESU engagement). Respondents from the legitimate stakeholder (i.e. Police) spoke about changes that reduced their urgency and impacted internal changes to their procedures (i.e. mandatory completion of EDP form). Respondents from the TS stakeholder that was perceived to have the least power within the TS (i.e. Social Service Agency) identified an unanticipated internal change that would improve the communication between them and the powerful stakeholder (i.e. InSted program). As these frontline observations of what has changed in the TS are consistent with the four specific changes that were implemented by management and the results of the analysis of the objective indicator data, we can say with a high level of confidence that the planned TS change impacted the TS.

Major conclusions of this research are that TS stakeholders may: (1) perceive a change that they wanted, (2) not perceive a change that they wanted, and (3) perceive a change that they did not mention they wanted. Table 59 summarizes these findings for each of the three TS stakeholders. To remind the reader, the four specific changes that were implemented include: (1) 
Police mental health training, (2) the InSted program, (3) an Intervention and Escalation policy, and (4) mandatory completion of EDP forms.

\begin{tabular}{|c|c|c|c|}
\hline & Hospital & Police & $\begin{array}{c}\text { Social Service } \\
\text { Agency }\end{array}$ \\
\hline \multirow{3}{*}{$\begin{array}{l}\text { Perceived the } \\
\text { change that they } \\
\text { mentioned they } \\
\text { wanted } \\
\text { (i.e. Got Desired } \\
\text { Change) }\end{array}$} & & $\begin{array}{l}\text { Increased } \\
\text { interorganizational } \\
\text { communication }\end{array}$ & $\begin{array}{l}\text { Increased } \\
\text { interorganizational } \\
\text { communication }\end{array}$ \\
\hline & $\begin{array}{l}\text { ED created new process } \\
\text { that expedites service for } \\
\text { PMIs who are brought in } \\
\text { by Police }\end{array}$ & $\begin{array}{l}\text { ED created new } \\
\text { process that expedites } \\
\text { service for PMIs who } \\
\text { are brought in by } \\
\text { Police }\end{array}$ & \\
\hline & $\begin{array}{l}\text { Increased MHESU } \\
\text { engagement }\end{array}$ & & \\
\hline $\begin{array}{l}\text { Did not perceive } \\
\text { the change that } \\
\text { they mentioned } \\
\text { they wanted } \\
\text { (i.e. Thwarted } \\
\text { Desires) }\end{array}$ & $\begin{array}{l}\text { Police mandate } \\
\text { completion of EDP form }\end{array}$ & & $\begin{array}{l}\text { Training Police to } \\
\text { apprehend PMIs } \\
\text { appropriately }\end{array}$ \\
\hline \multirow{3}{*}{$\begin{array}{c}\text { Perceived a } \\
\text { change that they } \\
\text { did not mention } \\
\text { they wanted } \\
\text { (i.e. Unanticipated } \\
\text { Change) }\end{array}$} & $\begin{array}{l}\text { Increased } \\
\text { interorganizational } \\
\text { communication }\end{array}$ & & \\
\hline & & $\begin{array}{l}\text { Police mandate } \\
\text { completion of EDP } \\
\text { form }\end{array}$ & \\
\hline & & & $\begin{array}{l}\text { Social Service Agency } \\
\text { created InSted program } \\
\text { support PMI }\end{array}$ \\
\hline
\end{tabular}

Table 59: Comparison of what TS stakeholders mentioned they wanted and perceived

These conclusions have numerous theoretical implications, particularly for those who study TS change and social identity theory. Our findings support research that argues TS stakeholders are more likely to act when they are able to share the costs associated with mobilization (Rowley \& Moldoveanu, 2003). This thesis case study provides an example of TS stakeholders 'mobilizing' to change their partners within the TS, as opposed to changing a party outside the TS. More specifically, our analysis indicates that all TS stakeholders changed 
themselves, and that the majority of TS stakeholders were able to get a desired change from their TS partner as a result of the planned TS change. Future research may benefit from exploring how TS stakeholders work together by conducting their own organizational changes.

Our data analysis also supports the idea that the majority of the TS stakeholders (1) did not perceive that they got the change they wanted (e.g. community support workers wanted police officers to have mental health training but did not think it happened) and (2) did perceive that their TS partners did get the changes that they wanted (e.g. Police perceived changes regarding the mandatory completion of EDP forms even though they did not mention they wanted it to happen). This finding is of relevance to social identity theorists. According to Tajfel \& Turner (1979), the fact that people may think in terms of ingroups ("us") and outgroups ("them") can lead to group conflict. In this thesis, it would appear that each TS stakeholder thinks of themselves as an ingroup that wants certain changes to benefit themselves, but thinks of their TS partners as outgroups that may benefit from their hard work. Future research may benefit from looking further into TS change by applying a social identity theory lens.

These findings also have implications for practitioners. More specifically, this analysis shows that TS stakeholders should cautiously enter joint change initiatives and be aware that although they might implement agreed upon changes they cannot guarantee that they will receive desired changes from their TS partners in return. It is recommended, therefore, that managers clearly articulate what they want, and that this message is communicated throughout all ranks within each of the TS stakeholders so that everyone knows what should change. 


\subsubsection{MAKING SENSE OF PLANNED TS CHANGE TYPOLOGY}

Our analysis of the linkage between Perceptions of What Has Changed in the TS and Perceptions of TS Stakeholder Relationships within the refined framework was guided by the following research question:

"How might TS stakeholders' perceptions of what has changed within the TS impact their perceptions of (a) power, (b) legitimacy, and (c) urgency within the TS six months after the implementation of the planned TS change?"

Initial analysis of our data relating to this issue illuminated the process by which TS stakeholders "made sense of the planned TS change". More specifically, our analysis implicated two separate factors in this sensemaking process: what the individual wanted to see changed within the TS and what the individual perceived had changed within the TS. Secondary analysis of the data suggests that how an individual "makes sense of the planned TS change" impacts perceived TS stakeholder relationships (e.g., perceptions of who has power, legitimacy, and urgency). These analyses lead us to refine our preliminary conceptual framework in 3 ways: (1) we removed the direct relationship between Perceptions of Desired TS Changes and Perceptions of What Has Changed in the TS, (2) we removed the direct relationship between Perceptions of What Has Changed in the TS and Perceptions of TS Stakeholder Relationships at Time 2, and (3) we added the typology of making sense of planned TS change to our model.

The typology shown in Table 40 enhances our understanding of how individuals make sense of a change. Consistent with Balogun and Johnson's (2005) theorization of the sensemaking process, this typology suggests that an individual's old schemata (e.g. their perceptions of what needed to change) and sensemaking triggers (e.g. their observations of the change intervention) combine to influence the development of new schemata and, thus, sensemaking. This thesis 
extends this body of literature in that it shifts the analytic lens from sensemaking about organizational change to sensemaking about TS change. As major differences across organizational boundaries are likely to influence sensemaking processes (Kaghan \& Lounsbury, 2006; Vermeulen \& Barkema, 2001), this research presented a unique opportunity to learn more about the different ways that individuals make sense of a change.

Our findings suggest that there are four sensemaking processes that individuals may use to make sense of a planned TS change. First, respondents who perceived that a change they wanted had been implemented (i.e. Got Desired Change) made sense of the planned TS change by thinking that the new behaviors benefited TS stakeholders. Second, respondents who did not perceive that the change that they wanted had been implemented (i.e. Thwarted Desires) made sense of the planned TS change by thinking that their partner's had erected organizational barriers that impeded the implementation of their desired change. Third, respondents who perceived changes in their own behavior that they did not mention they wanted (i.e. Unanticipated Organizational Change) made sense of the planned TS change by thinking that the new behaviors harmed them. Fourth, respondents who perceived changes in their TS partner's behavior that they did not mention they wanted (i.e. Unanticipated Partner Change) made sense of the planned TS change by thinking that the new behaviors benefited their TS stakeholder. Based on these findings, we present our first set of propositions:

1. a) Individuals in the Got Desired Change cell of the typology make sense of the planned TS change by perceiving cues that reinforce the idea that planned change is beneficial. 
1. b) Individuals in the Thwarted Desires cell of the typology make sense of the planned TS change by perceiving cues that reinforce the idea that planned changes are impeded by other groups.

1. c) Individuals in the Unanticipated Organizational Changes cell of the typology make sense of planned TS change by perceiving cues that reinforce the idea that the planned changes are harmful.

1. d) Individuals in the Unanticipated Partner Changes cell of the typology make sense of planned TS change by perceiving cues that reinforce the idea that the planned changes are beneficial.

Additionally, we draw two major conclusions from these findings. First, these findings indicate that how you make sense of a change depends on whether (or not) you wanted it and whether (or not) you perceived it. This conclusion is important for sensemaking theorists, as it provides empirical evidence to support the notion that wanting and perceiving a change are major predictors of sensemaking processes about change. We recommend future researchers examine what individuals say the want to see changed and what they perceive has changed when considering how they make sense of it.

Managers should also take note of this conclusion, as it suggests that managers should be aware of what their employees want from a change and perceived has changed over time if they want to understand how their employees might make sense of a change. Increased communication between managers and frontline employees may allow managers the opportunity to shape how their employees make sense of the change by influencing employees' perceptions of 'what is wanted' and 'what changed'. 
The second major conclusion associated with the making sense of planned TS change typology is that TS stakeholders may make sense of planned TS change in different ways than their TS partners. This finding is important for researchers, as they suggest that individuals belonging to different stakeholders have different sensemaking processes that guide their understanding of the change. This thesis also offers a typology for future sensemaking researchers to compare sensemaking processes of those who are employed at different organizations.

Managers and change consultants working within cross-sector collaborations and other joint change initiatives should also be aware of this finding, as it implies that individuals belonging to different groups may make sense of a change in very different ways than that of other parties. These practitioners should be aware that, while they may interpret a change from a positive perspective, there may be others from another stakeholder whom interpret the change from a negative perspective. To limit these competing understandings of the change, we recommend decision-makers, middle-managers, and frontline workers engage in greater formal and informal communication (Balogun, 2006) across organizational boundaries to facilitate interorganizational sensegiving at multiple levels (e.g., senior manager, middle-manager, frontline).

Lastly, the dimensions of the typology developed for this research identify four different ways that individuals may make sense of a planned TS change that may prove useful in future efforts to specify linkages between sensemaking at the individual level and change at the TS level. The findings suggest that individuals' sensemaking about a planned TS change may be influenced by the interplay between: (1) what the individual wanted to change in the TS, and (2) what the individual perceived had changed in the TS. Furthermore, the findings suggest that the individual's sensemaking process results in the development of different schemata (e.g. who benefitted from the change) that are likely to influence his/her perceptions of TS stakeholder 
relationships. Although it is beyond this research to examine the impact of these sensemaking processes beyond the level of the individual and his/her perceptions, the propositions advanced suggest inter-level linkages that might potentially enrich such a model. Refinement of the propositions, specification of the conditions under which they might pertain, and the development of measures and hypotheses for testing await future research.

\subsubsection{RELATIONSHIPS BETWEEN CONSTRUCTS IN THE REFINED THEORETICAL FRAMEWORK}

This section seeks to explain the relationships between the constructs within our refined theoretical framework. This section of the conclusion is divided into three subsections. The first subsection (Section 7.2.4.1) discusses the relationship within the model that was supported by data. Subsection two (Section 7.2.4.2) identifies the two relationships within the model that were not supported by data. Lastly, Section 7.2.4.3 discusses new relationships within the model that emerged from the analysis of the data.

\subsubsection{THEORETICAL RELATIONSHIPS SUPPORTED BY DATA}

The data analysis undertaken for this thesis supported only one of the three paths shown in our preliminary conceptual framework (see Figure 10). More specifically, our findings suggest that perceptions of TS stakeholder power, legitimacy, and urgency at Time 1 are related to perceptions of TS stakeholder power, legitimacy, and urgency at Time 2, respectively. For example, let's first consider perceptions of power and urgency within the TS. To explain the changes to perceptions of power and urgency, we draw from the physics literature to identify the parallel between power and urgency within a TS and mass within a system. According to the law of conservation of mass, the mass of any closed system must remain constant over time, as it is neither created nor destroyed (Sterner, Small, \& Hood, 2011). Within this thesis, we find that TS 
stakeholder power and urgency is also neither 'created' nor 'destroyed' over time, but may shift between TS partners in response to a planned TS change. More specifically, the data from our analysis suggests that the planned TS change shifted TS stakeholder power from the Hospital to the Police, and TS stakeholder urgency from the Police to the Hospital. This finding is interesting, as it suggests that a TS change that is driven by internal forces (i.e. planned TS change) cannot increase the power within the system, nor decrease the urgency within the system, even though that might be the goal of the change initiative. This would also imply that, within a planned TS change that is internally driven, there is less likely to be a mutual gain and more likely to be a zerosum game such that individual TS stakeholders win (e.g. those who gain power) or lose (e.g. those who gain urgency). Interestingly, according to the logic implied by the law of conservation of mass, TS changes that are driven by external forces (e.g. government legislation, new resources) may add or remove power or urgency from the system.

The results do not, however, suggest a zero-sum game when we consider changes to perceptions of legitimacy within the TS. Our analysis suggests that the legitimacy of the Police eroded in the eyes of healthcare workers (see Section 6.2.5.2 for more detail) because healthcare workers thought that the planned TS change favored the Police's situation over the Hospital's situation. More specifically, the data suggests that, while healthcare workers thought that the planned TS change resulted in Police spending less time in the Hospital, they also thought that the planned TS change resulted in Police apprehending more PMIs who did not belong in the Hospital post-change than pre-change. One resource nurse summed up his view of the Hospital-Police partnership throughout the planned TS change by saying: "I feel over this last year that we were on a date with the Police. We got taken for dinner, had a nice conversation, but then we had to pay for it." Based on these findings, we present our second set of propositions: 
2. a) Under conditions of planned TS change, as perceived power within the TS increases for one TS stakeholder, perceived power within the TS decreases for another TS stakeholder.

2. b) Under conditions of planned TS change, as perceived urgency within the TS increases for one TS stakeholder, perceived urgency within the TS decreases for another TS stakeholder.

2. c) TS stakeholder A's legitimacy erodes in the eyes of employees from TS stakeholder B when employees from TS stakeholder B perceive a planned TS change favors TS stakeholder A's situation over their own.

These findings support our conclusion that a planned TS change impacts perceptions of TS stakeholder power and urgency differently than it impacts perceptions of TS stakeholder legitimacy over time. This conclusion is particularly relevant for TS change and stakeholder researchers. First, it emphasizes the notion that, within a TS, there is stability in TS stakeholder power and urgency over time. For example, while a planned TS change may decrease a TS stakeholder's power, the power of another party within the TS is likely to increase. With regards to legitimacy, however, decreases in one party's legitimacy does not necessarily reflect increases in another party's legitimacy. Future researchers may benefit from exploring different types of changes (e.g. externally driven) that increase or decrease perceptions of power, legitimacy, and urgency in one TS stakeholder without affecting their TS partner.

These findings are also important for managers, as they suggest that one's benefit (in terms of gaining power or losing urgency) from a planned TS change comes at the cost of their partner. The results would also suggest that managers need to cautiously enter planned TS changes that are 
designed to benefit them because, if their partner thinks that they are the only party benefiting from the change, then they may be harmed (in terms of losing legitimacy) in the long run.

\subsubsection{THEORETICAL RELATIONSHIPS NOT SUPPORTED BY DATA}

Our data provided little evidence to support the linkages between Perceptions of Desired TS changes and Perceptions of What Has Changed in the TS, and between Perceptions of What Has Changed in the TS and Perceptions of TS Stakeholder Relationships at Time 2. While there was some overlap between what respondents wanted to see changed in the TS and what they perceived had changed in the TS, our analysis did not support a direct linkage between these two constructs. Instead, the findings of our analysis supported the idea that respondents' perceptions of desired and perceived changes contributed to how they made sense of the planned TS change. The results also indicate that individuals' sensemaking of a planned TS change influences how individuals feel each TS stakeholder was impacted by the change. See Section 5.3 for a detailed discussion of the making sense of planned TS change typology, or Section 7.2.3 for a high level discussion of conclusions regarding the making sense of planned TS change typology.

\subsubsection{RELATIONSHIPS WITHIN THE REFINED THEORETICAL FRAMEWORK EMERGING FROM DATA}

Our analysis identified the relationship between Perceptions of TS Stakeholder Relationships at Time 1 and Perceptions of Desired TS Changes at Time 1. Although it was not

our intent to explore the relationship between perceptions of TS stakeholder relationships and perceptions of desired TS changes in this thesis, the findings from our analysis support the notion that there was a relationship between respondents' perceptions of TS stakeholder relationships prechange and what they wanted to change within the TS. Our analysis suggests, for example, that TS stakeholders wished to change the aspect of the TS that gave power to the stakeholder who was 
perceived to have the most power (i.e. the doctor's ability to decide when Police leave the Hospital). They also indicated that TS stakeholders wanted to change the aspect of the TS that caused urgency to the stakeholder perceived to have the most urgency (i.e. Police leaving the Hospital promptly). Also, there is evidence to support the notion that respondents from both the powerful (i.e. Hospital) and powerless (i.e. Social Service Agency) stakeholders wanted the legitimate stakeholder (i.e. Police) to demonstrate greater legitimacy (i.e. Police apprehending PMIs under mental health act). We partially explain why this may occur within a TS by drawing from the public sector and stakeholder literatures. Public sector research argues that public sector organizations will only collaborate when they cannot get what they want without collaborating (Bryson et al., 2006; Hudson et al., 1999; Robers, 2001). However, Mitchell et al.'s (1997) research suggests that, within the context of a TS, organizations are more likely to collaborate (i.e. respond to their partners) when they perceive their partners have power, legitimacy, and urgency. Thus, as public sector organizations attempt to avoid future situations involving collaboration, they may want to change the TS in such a way that they do not perceive their partners having power, legitimacy, and urgency. Based on this finding, we present our third set of propositions:

3. a) TS stakeholders' perceptions of power within the TS impact their views of what they want to change in the TS.

3. b) TS stakeholders' perceptions of legitimacy within the TS impact their views of what they want to change in the TS.

3. c) TS stakeholders' perceptions of urgency within the TS impact their views of what they want to change in the TS.

The above findings support our conclusion that perceptions of TS stakeholder power, legitimacy, and urgency can impact what TS stakeholders want to see changed within the TS. This 
conclusion is important for researchers, as it suggests that perceptions of TS stakeholder relationships can predict what TS stakeholders want to change in the TS. It may also be helpful to managers participating in TS changes, as it may remind them what they should consider when planning a change. In other words, have they considered how their power, legitimacy, and urgency may be affected by the implementation of a planned TS change?

It should be noted that this thesis provides some evidence to support the addition of two other relationships between constructs within our model, however, we believe that further study is required before we do so. For example, while we did not intend to explore the relationship between the views of what respondents wanted to change within the TS and the four specific changes that decision-makers implemented, the results of this thesis show strong similarities between the two. The changes that respondents mentioned they wanted to see in the TS corresponded with three of the four specific changes that were implemented (i.e., the intervention and escalation policy, Police mental health training, and mandatory completion of an EDP form). Future research may confirm this theoretical linkage by investigating the relationship between the views of decision-makers and frontline workers.

Similarly, while we did not intend to examine whether the views of TS stakeholder relationships at Time 1 drove the planned TS change itself, the results of this thesis suggest that they might be related to why the change occurred in the first place. Consistent with Mitchell et al.'s (1997) theory of stakeholder salience, which suggests that organizations are more likely to respond to their partners whom they perceive have power, legitimacy, and urgency, this case study involves parties who are perceived to have power, legitimacy, and urgency responding to each other in the form of a planned TS change. More specifically, before the planned TS change, the TS involved a legitimate stakeholder with high urgency waiting for a powerful partner to respond, 
and a powerful partner exerting power over their partners without experiencing any penalty. Unfortunately, as we did not interview decision-makers, we do not know whether their views of TS stakeholder relationships are: (1) consistent with those opinions from frontline employees, and (2) linked to the reasons why they started the planned TS change in the first place. Future research may benefit from confirming this theoretical linkage by investigating the relationship between the views of frontline employees, the views of decision-makers, and the reasons why decision-makers started the planned TS change.

\subsection{CONTRIBUTIONS}

This research contributes to change theory in two primary ways. First, this thesis develops a framework describing how a planned TS change may impact perceptions of TS stakeholder relationships over time. This framework may act as a guide for future research on the topic of TS change, and facilitate a comparison of how change takes place in an organization and how change takes place in a TS. Second, this research also contributes to the change literature by offering a typology of making sense of planned TS change. This typology suggests an area of the change literature that requires further attention, as it indicates that individuals' perceptions of what they want to change and what they think has changed can impact their sensemaking processes about change initiatives.

This research enriches the TS literature by examining interorganizational relationships from a stakeholder perspective. In so doing, this research shows that a planned TS change can impact perceptions of interoganizational power, legitimacy, and urgency, and suggests the need for future researchers to explore how planned changes in a system can affect other dimensions of interorganizational relationships. This research also contributes to the TS literature by developing a conceptual model of 'sharedness' within a TS (see Figure 9) and a tool for operationalizing 
'sharedness' within a TS (see Figure 7). Accordingly, this research emphasizes the importance of 'sharedness' within the dynamics of TSs, and provides the appropriate tools needed for future researchers to explore 'sharedness' within other TSs. Lastly, this research also supports the call for more empirical research that delves into TSs that change without adding new or removing old organizations (e.g. Koka et al., 2006).

Lastly, this research contributes to the stakeholder literature by examining stakeholder perceptions over time. In contrast to much of the stakeholder literature, which has approached stakeholders from a static perspective (Key, 1999), this research provides evidence that stakeholders may become more or less powerful, legitimate, or urgent over time to their TS partners. This research supports the call for future stakeholder research to contribute to a dynamic understanding of stakeholders (Mainardes et al., 2011) by considering stakeholders over time. This thesis also contributes to the stakeholder literature by providing empirical support for the linkage between TS stakeholders' perceptions of power, legitimacy, and urgency and what they want to change in the TS. Future research may benefit from using quantitative methods to test the propositions associated within this linkage.

\subsection{LIMITATIONS AND DIRECTIONS FOR FUTURE RESEARCH}

Despite the theoretical contributions of the thesis research, there were several limitations of this research that suggest directions for future research. The first limitation arises from the decision to use a case study methodology to examine the planned TS change. The case study approach may limit the ability to predict TS change beyond the limits of the Police, Hospital, and Social Service Agency interface. The intention of this thesis is to increase our understanding of how the planned TS change may have impacted TS stakeholder relationships, and not make statistical generalizations to a wider population. This focus is consistent with the opinions of 
leading scholars in the area that suggest the transferability of qualitative research (Shah \& Corley, 2006). More specifically, Yin (2009, p. 15) asserts case studies are "generalizable to theoretical propositions and not to populations," while Patton (2002) claims it is more important to do justice to the cases under study than to look for similarities with other situations. Using theory developed in this research in another TS facing change is recommended for future studies of TSs and TS stakeholder relationships.

A second limitation of the current thesis research stems from the choice to conceptualize TS stakeholder relationships using stakeholder attributes as identified in Mitchell et al.'s (1997) stakeholder classification. Other more general interorganizational relationship (e.g. Uzzi, 1997) or stakeholder models (e.g., Kaler, 2002; Atkinson at al., 1997) exist, each of which may be equally suitable for the examination of TS stakeholder relationships over time. While it is beyond the scope of this research to include other interorganizational relationship or stakeholder frameworks, future research may benefit from using the approach taken in this study to investigate the impact of planned TS change on other TS stakeholder relationship frameworks.

Third, participants in this study are from three public sector organizations. As shown in the literature review, there are substantial differences between private and public sector organizations. Future studies should focus on undertaking this type of study in private organizations where organizational and TS change may be quite different.

Fourth, data from respondents employed in TS stakeholders within in multiple public sector industries (i.e., policing, healthcare, social service) were used to assemble the dataset for our case study. As our literature review identified little research exploring differences between TSs consisting of public sector firms, we are unsure whether TSs composed of stakeholders belonging to different public sector industries behave differently than TSs composed of stakeholders 
belonging to the same public sector industry (e.g., policing, healthcare) in the context of change. Future researchers could remove this potential limitation by focusing on TS stakeholders from a single public sector industry (e.g. multiple police services working together).

Fifth, there are many possible stakeholders, or sub-stakeholders, not considered in this research. While this research focuses on TS change from the perspective of the three TS stakeholders believed to have the greatest influence on each other and the TS, it is possible that other stakeholders or sub-stakeholders may contribute to how planned change unfolds in this particular TS over time. For example, since government legislation (i.e. mental health act) is mandating the Police and Hospital to participate in the PMI process, it is possible that the government may also be considered a stakeholder that affects how planned change unfolds in this particular TS. Also, as we interviewed only 25 respondents per TS stakeholder at Time 1 and 20 respondents per TS stakeholder at Time 2, there is likely to be a significant amount of diversity across as well as within TS stakeholders that may be missed by only interviewing 25/20 people per TS stakeholder. Future researchers could remove (or reduce) this limitation by conducting similar studies with larger sample sizes relative to the scale of the TS stakeholders, and more diverse samples to account for different stakeholders and sub-stakeholder groups. As this research has found that 'sharedness' across organizational boundaries may impact how change unfolds in a TS, it is also possible that 'sharedness' within organizational boundaries may impact how change unfolds in a TS.

The sixth and seventh limitation of this research are associated with the data collection undertaken for this thesis. First, we faced significant challenges collecting longitudinal data from the Social Service Agency. We were only able to interview 9 respondents at Time 1 and at Time 2. The implications of this is that we were unable to analyze differences in individuals' views 
because of differences in the sample. Future studies may benefit from collecting data from respondents who can be interviewed at multiple points in time. Second, we were challenged to collect longitudinal interview data from the PMI him/herself. The PMI is an important stakeholder in the PMI process. While the input of the PMI might have resulted in more in-depth understanding of the PMI process experts in the area (police, health care providers, and community support workers in the PMI process) told us that it would be very unlikely that any PMI we interviewed prior to the introduction of the planned TS change would also experience the process within the first six months after the change. They also noted that the PMI process is meant to stabilize individuals who are perceived to be endangering themselves or others and indicated that although some "frequent fliers" are seen all the time, most PMIs avail themselves of the service only in times of crisis. Finally, they observed that PMIs who are picked up and brought involuntarily to the hospital because of mental health issues might not be the best people to interview with respect to what they had observed had changed. Future research could address this issue by asking PMI's to reflect back on visits prior to change and asking them if they noticed any differences or by asking family members their observations.

Eighth, this study explores how planned TS change may impact TS stakeholder relationships over time by using three theoretical lenses: change, TS, and stakeholder theory. In so doing, this study does not include other factors that may influence the process of change within TSs. For instance, this research does not directly investigate capacity to change (e.g. Maitlis \& Sonenshein, 2010). Instead, it indirectly investigates capacity to change by considering the power dynamics within the TS, such that those TS stakeholders who have power are more capable of implementing change within the TS (Van de Ven \& Poole, 1995). Another example of this issue is that research does not specifically look at leadership within each of the TS stakeholders or 
leadership within the TS, even though previous research has argued that leadership is instrumental to change initiatives (Nadler \& Tushman, 1989). Given that leaders may be gatekeepers to the change process, it is possible that they are the key players that may affect change at the TS level. Lastly, this research does not directly consider factors that influence conformity. There is some research in the group dynamics literature that suggests conformity is a function of culture, age, group size, status, prior commitment, and level of group cohesion (e.g., Asch, 1956; Solomon, 1987). Future research may benefit from considering additional factors such as capacity to change, leadership, and conformity within the TS.

The final limitation is associated with the archival data. Although archival data may be free of some bias that may be found in other data collection methods, it too may be biased by the organization collecting the data (Singleton \& Straights, 2010). As the research includes archival data collected from both police and hospital organizations, it is possible that this data is biased. Triangulation of archival data from police and hospital organizations and interview data from the Police, Hospital, and Social Service Agency will help improve the accuracy of the data collected and the quality of research produced.

One promising avenue for future research is to test the external validity of the concepts and theoretical linkages identified within the framework by examining the transferability to other populations and settings. At the most basic level, this might be done by examining other TSs and replicating the interviews with other samples, including those from the private sector. The goal of further qualitative research at this point would be to determine whether the new data fit the conceptual framework emerging from this study, or whether the framework requires further refinement. Once confident that the constructs have been adequately defined, researchers might 
move toward the development of measures of the constructs and the formulation of hypotheses for quantitative testing.

It would also be interesting to see how the theoretical framework developed in this thesis applies to changes that are externally driven by a third party. This case study revolved around a planned TS change that was internally driven, and resulted in the shifting of power and urgency from one TS stakeholder to another. Perhaps in an externally motivated change, new resources may become more (or less) available (Van de Ven \& Poole, 1995). This shift in resources could impact the amount of power or urgency within the TS. Or another external change might be a change in legislation, which could result in shifting the legitimacy of each of the key players within the TS. In any case, it would be interesting to examine the similarities and differences between internally and externally driven TS changes in our theoretical framework. 


\section{CHAPTER 8: REFERENCES}

Abolafia, M. Y. (2010). Narrative construction as sensemaking: How a central bank thinks. Organization Studies, 31(3), 349-367.

Abrahamson, E., \& Fombrun, C. J. (1994). Macrocultures: De-terminants and consequences. Academy of Management Review, 19, 728-755.

Adler, R. M., \& Koehn, D. J. (2012). CALM: Complex Adaptive System (CAS)-Based Decision Support for Enabling Organizational Change. In Unifying Themes in Complex Systems VII: Proceedings of the Seventh International Conference on Complex Systems (pp. 2-13). Springer Berlin Heidelberg.

Agle, B. R., Mitchell, R. K., \& Sonnenfeld, J. A. (1999). Who Matters to Ceos? An Investigation of Stakeholder Attributes and Salience, Corpate Performance, and Ceo Values. Academy of management journal, 42(5), 507-525.

Ahuja, G., Soda, G., \& Zaheer, A. (2012). The genesis and dynamics of organizational networks. Organization Science, 23(2), 434-448.

Allen, B. A., Juillet, L., Paquet, G., \& Roy, J. (2001). E-Governance \& government on-line in Canada: Partnerships, people \& prospects. Government Information Quarterly, 18(2), 93104.

Andersson LM, Bateman TS. (2000). Individual environmental initiative: championing natural environmental issues in U.S. business organizations. Academy of Management Journal 43: $548-571$.

Anderson, E., \& Jap, S. (2012). The dark side of close relationships. Sloan Management. 
Anderson, P., \& Tushman, M. L. (2001). Organizational environments and industry exit: The effects of uncertainty, munificence and complexity. Industrial and Corporate Change, $10(3), 675-711$.

Ang, S., Van Dyne, L., \& Begley, T. M. (2003). The employment relationships of foreign workers versus local employees: A field study of organizational justice, job satisfaction, performance, and OCB. Journal of Organizational Behavior, 24(5), 561-583.

Argyris, C. (1993). Knowledge for action: A guide to overcoming barriers to organizational change. Jossey-Bass Inc., Publishers, 350 Sansome Street, San Francisco, CA 94104.

Armenakis, A. \& Bedeian, A. (1999). Organizational change: A review of theory and research in the 1990s'. Journal of Management, 25 (3), p. 293 - 315.

Armenakis, A., Harris, S. \& Field, H. (1999). Making change permanent: A model for institutionalizing change interventions. In W. Passmore \& R. Woodman (Eds.), Research in organizational change and development, Stanford, ST: JAI Press Inc.

Armenakis, A. A., Harris, S. G., \& Mossholder, K. W. (1993). Creating readiness for organizational change. Human relations, 46(6), 681-703.

Aronson, J., \& Sammon, S. (2000). PRACTICE AMID SOCIAL SERVICE CUTS AND RESTRUCTURING: Working with the Contradictions of" Small Victories". Canadian Social Work Review/Revue canadienne de service social, 167-187.

Arora S, Cason T. (1999). Do community characteristics influence environmental outcomes? Evidence from the Toxic Release Inventory. Southern Economic Journal 65: 691-716.

Ashforth, B. E., \& Mael, F. A. (1998). The power of resistance: Sustaining valued identities. Power and influence in organizations, 89, 119. 
Atkinson, A. A., Waterhouse, J. H., \& Wells, R. B. (1997). A stakeholder approach to strategic performance measurement. Sloan management review, 38(3), 25-37.

Ayliffe, L., Lagrace, C., Muldoon, P. (2005). The use of a mental health triage assessment tool in a busy Canadian tertiary care children's hospital. Journal of Emergency Nurses, 31, p.1615.

Babbie, E. (2001). Qualitative field research. Babbie, ER, The Practice of Qualitative Research.

Bachmann, R. (2001). Trust, power and control in trans-organizational relations. Organization studies, 22(2), 337-365.

Bachmann, R. (2003). The coordination of relations across organizational boundaries. International Studies of Management \& Organization, 33(2), 7-21.

Back, K. W. (1992). 'This business of topology'. Journal of Social Issues, 48, 2, 51-66.

Bailey, C. (2007). A Guide to Qualitative Field Research, Second Edition. Thousand Oaks, CA: Pine Forge Press.

Balogun, J. (2003). From blaming the middle to harnessing its potential: Creating change intermediaries. British Journal of Management, 14(1), 69-83.

Balogun, J. (2006). Managing change: Steering a course between intended strategies and unanticipated outcomes. Long Range Planning, 39(1), 29-49.

Balogun, J., Bartunek, J. M., \& Do, B. (2015). Senior managers' sensemaking and responses to strategic change. Organization Science, 26(4), 960-979.

Balogun, J., \& Johnson, G. (2004). Organizational restructuring and middle manager sensemaking. Academy of management journal, 47(4), 523-549.

Balogun, J., \& Johnson, G. (2005). From intended strategies to unintended outcomes: The impact of change recipient sensemaking. Organization studies, 26(11), 1573-1601. 
Balogun, J. (2006). Managing change: Steering a course between intended strategies and unanticipated outcomes. Long Range Planning, 39(1), 29-49.

Bamford, D. R., \& Forrester, P. L. (2003). Managing planned and emergent change within an operations management environment. International Journal of Operations \& Production Management, 23(5), 546-564.

Bartunek, J. M., Rousseau, D. M., Rudolph, J. W., \& DePalma, J. A. (2006). On the receiving end sensemaking, emotion, and assessments of an organizational change initiated by others. The Journal of applied behavioral science, 42(2), 182-206.

Battilana, J., \& Casciaro, T. (2012). Change agents, networks, and institutions: a contingency theory of organizational change. Academy of Management Journal, 55(2), 381-398.

Baum, J. A. C., B. McEvily, T. J. Rowley. 2012. Better with age? Tie longevity and the performance implications of bridging and closure. Organization Science, 23(2) 529-546.

Baxter, L. A., \& Braithwaite, D. O. (Eds.). (2008). Engaging theories in interpersonal communication: Multiple perspectives. Sage Publications.

Bayley, D. H. (2008). Police reform: Who done it?. Policing \& Society, 18(1), 7-17.

Beach, S. (2008). Sustainability of network governance: stakeholder influence. Proceedings of Contemporary Issues in Public Management: The 12th Annual Conference of the International Research Society for Public Management (IRSPM XII), Brisbane, pp. 1-23.

Bennett, W. L. (2004). Branded political communication: Lifestyle politics, logo campaigns, and the rise of global citizenship. Politics, products and markets: Exploring political consumerism, past and present, 101-125.

Bhasin, S., \& Burcher, P. (2006). Lean viewed as a philosophy. Journal of Manufacturing Technology Management, 17(1), 56-72. 
Bingham, C. B., \& Eisenhardt, K. M. (2006). Unveiling the creation and content of strategic processes: How and what firms learn from heterogeneous experience. Proceedings of the Academy of Management.

Blau, P. M. (1994). Structural Contexts of Opportunities. University of Chicago Press, Chicago.

Boje, D., \& Rosile, G. A. (2003). Comparison of socio-economic and other transorganizational development methods. Journal of Organizational Change Management, 16(1), 10-20.

Bovaird, T. (2005). Public governance: balancing stakeholder power in a network society. International Review of Administrative Sciences, 71(2), 217-228.

Bozan, M.G. (2003). Using Lewin's force field analysis in implementing a nursing information system. CIM: Computers, Informatics, Nursing, 21, pp. 80-85.

Bridges, W. (2003). Managing transitions: Making the most of change, Second Edition. Cambridge, MA: Perseus Publishing.

Brodbeck, F. C., Kerschreiter, R., Mojzisch, A., Frey, D., \& Schulz-Hardt, S. (2002). The dissemination of critical, unshared information in decision-making groups: The effects of pre-discussion dissent. European Journal of Social Psychology, 32(1), 35-56.

Brown, L. D. (1980). Planned change in under-organized systems. Systems theory for organization development, 193, 210.

Brown, S., Eisenhardt, K. (1998). Competing on the edge: Strategy as structured chaos. Boston: Harvard Business School Press.

Bruns, H. C. (2013). Working alone together: Coordination changes expert practice in crossdomain collaboration. Academy of Management Journal, 56: 62-83.

Bryson, J. M. (2004). What to do when stakeholders matter: stakeholder identification and analysis techniques. Public management review, 6(1), 21-53. 
Bryson, J. M., \& Crosby, B. C. (2006). Leadership for the common good. Creating a culture of collaboration, 367-396.

Buchholz, R. and Rosenthal, S. (2005). Toward a contemporary conceptual framework for stakeholder theory, Journal of Business Ethics, 58 (1), pp. 137-48.

Bundy, J., Shropshire, C., Buchholtz, A. (2013). Strategic cognition and issue salience: Toward an explanation of firm responsiveness to stakeholder concerns. Academy of Management Review, 38 (3), p. $352-376$.

Burke, W. W. (2010). Organization change: Theory and practice. Sage.

Burke, W., \& Litwin, G. (1992). A causal model of organizational performance and change. Journal of Management, 18: 523-545.

Burnes, B. (1996). No such thing as... a "one best way" to manage organizational change. Management Decision, 34(10), 11-18.

Burnes, B. (2004). Kurt Lewin and the Planned Approach to Change: A Re-appraisal. Journal of Management studies, 41(6), 977-1002.

Burnes, B., \& Cooke, B. (2012). Kurt Lewin's Field Theory: A Review and Re-evaluation. International Journal of Management Reviews.

Burnes, B., \& Cooke, B. (2012). Review article: The past, present and future of organization development: Taking the long view. Human Relations, 65(11), 1395-1429.

Burt, R. S. (2005). Brokerage and Closure: An Introduction to Social Capital. Oxford University Press, Oxford, UK.

Boyne, G. A. (2002). Public and private management: what's the difference?. Journal of management studies, 39(1), 97-122. 
Bryson, J. M. (2011). Strategic planning for public and nonprofit organizations: A guide to strengthening and sustaining organizational achievement (Vol. 1). John Wiley \& Sons.

Caldwell, B. J. (2009). Centralisation and Decentralisation in Education: A New Dimension to Policy. In Decentralisation, school-based management, and quality (pp. 53-66). Springer Netherlands.

Callender, H., Chandrasekera, U., Lillie, P. and McGurk, T. (2013). Strategies for Implementing Effective Police-Emergency Department Protocols in Ontario. HSJCC webinar. Accessed February 3, 2014 from https://camh.adobeconnect.com/_a829238269/p5en5c7ktpd/? launcher $=$ false $\&$ fcsContent $=$ true $\& p b M o d e=$ normal.

Calo, T. (2005). The generativity track: A transitional approach to retirement. Public Personnel Management, 34 (4), p. $301-312$.

Caloghirou, Y., Ioannides, S., \& Vonortas, N. S. (2003). Research joint ventures. Journal of Economic Surveys, 17(4), 541-570.

Capaldo, A. (2007). Network structure and innovation: The leveraging of a dual network as a distinctive relational capability. Strategic management journal, 28(6), 585-608.

Carroll, A. (1996), Business and Society: Ethics and Stakeholder Management, 3rd ed. SouthWestern, Cincinnati, OH.

CELANI, A., \& TASA, K. (2010, August). WE'RE ALL IN THIS TOGETHER: EXAMINING ASSOCIATIONS BETWEEN COLLECTIVISTIC GROUP NORMS, COLLECTIVE EFFICACY AND TEAM PERFORMANCE. In Academy of Management Proceedings (Vol. 2010, No. 1, pp. 1-6). Academy of Management.

Chae, M. S., \& Hill, J. S. (2000). Determinants and benefits of global strategic marketing planning formality. International Marketing Review, 17(6), 538-563. 
Charmaz, K. (2006). Constructing grounded theory: A practical guide through qualitative analysis. California: Sage.

Checa, N., Maguire, J. \& Barney, J. (2003). The new world disorder. Harvard Business Review, August, p. $70-79$.

Chia, R. (1999). A 'rhizomic'model of organizational change and transformation: Perspective from a metaphysics of change. British journal of management, 10(3), 209-227.

Chia, R., \& Langley, A. (2004). The First Organization Studies Summer Workshop on Theorizing Process in Organizational Research. June 12-13 2005, Santorini, Greece: Call for papers. http://www.egosnet.org/journal/os_summer_workshop.html

Christiansen, C. R. (2006). Sense-making and entrepreneurial coalition building: a case of competing interests, cultural barriers, and interorganizational relations in a nonprofit health plan. Intl Journal of Public Administration, 29(7), 501-515.

Clarke, N. (2005). Transorganization development for network building. The Journal of Applied Behavioral Science, 41(1), 30-46.

Clark, T., \& Salaman, G. (1998). Telling tales: management gurus' narratives and the construction of managerial identity. Journal of Management studies, 35(2), 137-161.

Clarkson, M. (Ed.). (1998). The corporation and its stakeholders: classic and contemporary readings. Toronto: University of Toronto Press.

Coch, L., \& French Jr, J. R. (1948). Overcoming resistance to change. Human relations.

Coleman, J. S. (1988). Social capital in the creation of human capital. American Journal of Sociology. 94(Supplement) S95-S120. 
Coleman, T. G., \& Cotton, D. (2010). Police interactions with persons with a mental illness: Police learning in the environment of contemporary policing. Ottawa: Mental Health Commission of Canada.

Collins, H., \& Evans, R. (2007). Rethinking expertise. Chicago: University of Chicago Press.

Comeche, J. and Loras, J. (2010), "The influence of variables of attitude on collective entrepreneurship", International Entrepreneurship and Management Journal, Vol. 6 No. 1, pp. 23-38.

Cooke, B. (1999). 'Writing the left out of management theory: the historiography of the management of change'. Organization, 6, 1, 81-105.

Cook, K. S., Cheshire, C., Rice, E. R., \& Nakagawa, S. (2013). Social exchange theory (pp. 6188). Springer Netherlands.

Cotton, D., \& Coleman, T. G. (2010). Canadian police agencies and their interactions with persons with a mental illness: A systems approach. Police Practice and Research: An International Journal, 11(4), 301-314.

Cox, T. (1994). Cultural diversity in organizations: Theory, research and practice. BerrettKoehler Publishers.

Creswell, J. (1998). Qualitative inquiry and research design: Choosing among five traditions. Thousand Oaks, CA: Sage.

Cronin, M. A., \& Weingart, L. R. (2007). Representational gaps, information processing, and conflict in functionally diverse teams. Academy of Management Review, 32(3), 761-773.

Crosby, B. C., \& Bryson, J. M. (2005). A leadership framework for cross-sector collaboration. Public Management Review, 7(2), 177-201. 
Cummings, T. G., Blumenthal, J. F., \& Greiner, L. E. (1983). Managing organizational decline: The case for transorganizational systems. Human Resource Management, 22(4), 377-390.

Cummins, T. \& Worley, C. (1997). Managing change. In Organizational development and change, Seventh Edition, Cincinnati: South-West College Publishing.

Cummings, T. G., \& Worley, C. G. (2009). Organization development and change. Cengage Learning.

Das, T. K., \& Teng, B. S. (2000). A resource-based theory of strategic alliances. Journal of management, 26(1), 31-61.

Dawson, P. (2014). Temporal practices: time and ethnographic research in changing organizations. Journal of Organizational Ethnography, 3(2), 130-151.

Davis, K. (1973). The case for and against business assumption of social responsibilities. Academy of Management journal, 16(2), 312-322.

del Val, M. P., \& Fuentes, C. M. (2003). Resistance to change: a literature review and empirical study. Management Decision, 41(2), 148-155.

den Hond, F., de Bakker, F. (2007). Ideologically motivated activism: how activist groups influence corporate social change activities, Academy of Management Review, 32 (3), p. $901-924$.

Denis, J. L., Langley, A., \& Sergi, V. (2012). Leadership in the plural. The Academy of Management Annals, 6(1), 211-283.

Dent, E. B. and Goldberg, S. G. (1999). 'Challenging resistance to change'. Journal of Applied Behavioral Science, 35, 1, 25-41.

Donaldson, T., \& Preston, L. E. (1995). The stakeholder theory of the corporation: Concepts, evidence, and implications. Academy of management Review, 20(1), 65-91. 
Driscoll, C., \& Crombie, A. (2001). Stakeholder legitimacy management and the qualified good neighbor: The case of Nova Nada and JDI. Business \& Society, 40(4), 442-471.

Driscoll, C., \& Starik, M. (2004). The primordial stakeholder: Advancing the conceptual consideration of stakeholder status for the natural environment. Journal of Business Ethics, 49(1), 55-73.

Dror, I., Busemeyer, J., \& Basola, B. (1999). Decision making under time pressure: an independent test of sequential sampling models. Memory and Cognition 27: 713-725

Drori, I., \& Honig, B. (2013). A process model of internal and external legitimacy. Organization Studies, 34(3), 345-376.

Dutton, J. E., Ashford, S. J., O'Neill, R. M., \& Lawrence, K. A. (2001). Moves that matter: Issue selling and organizational change. Academy of Management Journal, 44(4), 716-736.

Dyer, J. H., Kale, P., \& Singh, H. (2001). Strategic alliances work. MIT Sloan Management Review, 37-43.

Eden, C. \& Ackermann, F. (1998). Making Strategy: The Journey of Strategic Management. London: Sage Publications.

Edmondson, A. C., \& McManus, S. E. (2007). Methodological fit in management field research. Academy of management review, 32(4), 1246-1264.

Eesley, C. \& Lenox, M. J. (2006). Firm Responses to Secondary Stakeholder Action. Strategic Management Journal, 27, p. 765-781.

Eisenhardt, K. (1989). Building theories from case study research. Academy of Management Review, 14 (4), p. $532-550$.

Eisenhardt, K. M., \& Graebner, M. E. (2007). Theory building from cases: opportunities and challenges. Academy of management journal, 50(1), 25-32. 
Elie-Dit-Cosaque, C., Pallud, J., \& Kalika, M. (2011). The influence of individual, contextual, and social factors on perceived behavioral control of information technology: A field theory approach. Journal of Management Information Systems, 28(3), 201-234.

Elsbach, K. D., \& Kramer, R. M. (2003). Assessing creativity in Hollywood pitch meetings: Evidence for a dualprocess model of creativity judgments. Academy of Management Journal, 46: 283-301.

Emirbayer, M., A. Mische. (1998). What is agency? American Journal of Sociology, 103(4) 9621023.

Engestrom, Y., Kerosuo, H., and Kajamaa, A. (2007). Beyond discontinuity: Expansive organizational learning remembered. Management Learning, 38(5), 319-336.

Evan, W. and Freeman, R. (1988), “A stakeholder theory of the modern corporation: Kantian capitalism", in Beauchamp, T. and Bowie, N. (Eds), Ethical Theory and Business, 2nd ed., Prentice-Hall, Englewood Cliffs, NJ, pp. 75-84.

Feldman, M. S. (2004). Resources in emerging structures and processes of change. Organization Science, 15(3), 295-309.

Fiss, P. C., \& Zajac, E. J. (2006). The symbolic management of strategic change: Sensegiving via framing and decoupling. Academy of Management Journal, 49(6), 1173-1193.

Ford, J. \& Ford, L. (1994). Logics of identity, contradiction, and attraction in change. Academy of Management Review, 19, p.756 - 785 .

Ford, J. D., Ford, L. W., \& D'Amelio, A. (2008). Resistance to change: The rest of the story. Academy of Management Review, 33(2), 362-377.

Forsythe, S. M. (1990). Effect of applicant's clothing on interviewer's decision to hire. Journal of Applied Social Psychology, 20(19), 1579-1595. 
Fowler, F. J. (1995). Improving survey questions: Design and evaluation (Vol. 38). Sage.

Freeman, R. E. (1984). Stakeholder management: framework and philosophy. Pitman, Mansfield, $M A$.

Freeman, R. (1998). A stakeholder's theory of the modern corporation. In Hartman, L. (Ed.), Perspectives in Business Ethics, McGraw-Hill, New York, NY, pp. 12-19.

Freeman, R. E. (1994). The politics of stakeholder theory: Some future directions. Business ethics quarterly, 409-421.

Freeman, R., \& McVea, J. (2001). A stakeholder approach to strategic management.

Freeman. R., \& Reed, D. L. (1983). Stockholders and stakeholders: A new perspective in corporate governance. California management review, 25, 88-106.

Friedman, A. and Miles, S. (2006). Stakeholders: Theory and Practice. Oxford University Press, Oxford.

Frooman, J. (1999). Stakeholders influence strategies. Academy of Management Review. 24 (2), pp. 191-205.

Galaskiewicz, J. (1985). Interorganizational relations. Annual review of sociology, 281-304.

Galpin, T. (1996). The human side of change: A practical guide to organization redesign. San Francisco: Jossey-Bass.

Gazley, B. (2008). Beyond the contract: The scope and nature of informal government-nonprofit partnerships. Public administration review, 68(1), 141-154.

Gersick, C. (1991). Revolutionary change theories: A multilevel exploration of the punctuated equilibrium paradigm. Academy of Management Review, 16(1), 10-36. 
Gibson, C. B., \& Earley, P. C. (2007). Collective Cognition in Action: Accumulation, Interaction, Examination, and Accommodation in the Development and Operation of Group Efficacy Beliefs in the Workplace. Academy of management review, 32(2), 438-458.

Gilmartin, M. J., \& D'Aunno, T. A. (2007). 8 Leadership Research in Healthcare: A Review and Roadmap. The Academy of Management Annals, 1(1), 387-438.

Gimeno, J. (2004). Competition within and between networks: The contingent effect of competitive embeddedness on alliance formation. Academy of Management Journal, 47(6), $820-842$.

Gioia, D. A., \& Thomas, J. B. (1996). Identity, image, and issue interpretation: Sensemaking during strategic change in academia. Administrative science quarterly, 370-403.

Goldsmith, W. W. (1999). Resisting the reality of race: land use, social justice and the metropolitan economy. Lincoln Institute of Land Policy.

Goodstein, L., Burke, W. (1991). Creating successful organizational change. Organizational Dynamics, p. $5-17$.

Goulding, C. (2002). Grounded Theory: A practical guide for management, business and market researchers. Thousand Oaks, California: Sage.

Government of Ontario (2012). Ontario's action plan for health care (ISBN 978-1-4435-8942-0). Queen's Printer for Ontario. Ministry of Health and Long Term Care.

Graebner, M. E. (2004). Momentum and serendipity: How acquired leaders create value in the integration of technology firms. Strategic Management Journal, 25: 751-777.

Gray, B. (2000). Assessing interorganizational collaboration: Multiple conceptions and multiple methods. Cooperative strategy: Economic, business, and organizational issues, 243-260. 
Greenwood, R. \& Hinings, C. (1996). Understanding radical organizational change: Bringing together the old and the new institutionalism. Academy of Management Review, 21 (4), p. $1022-1054$.

Greenwood, R., \& Suddaby, R. (2006). Institutional entrepreneurship in mature fields: The Big Five accounting firms. Academy of Management Journal, 49: 27-48.

Greenwood, R., Suddaby, R., \& Hinings, C. R. (2002). Theorizing change: The role of professional associations in the transformation of institutionalized fields. Academy of Management Journal, 45: 58-80.

Grimshaw, D., Vincent, S., \& Willmott, H. (2002). Going privately: partnership and outsourcing in UK public services. Public Administration, 80(3), 475-502.

GRUTTERINK, H., VAN DER, G. S., MOLlEMAN, E., \& JEHN, K. A. (2010, August). FEELING KNOWN: A MULTI-LEVEL EXAMINATION OF PERCEIVED EXPERTISE AFFIRMATION IN WORK TEAMS. In Academy of Management Proceedings (Vol. 2010, No. 1, pp. 1-6). Academy of Management.

Gulati, R. (1998). Network location and learning: The influence of network resources and firm capabilities on alliance formation. Strategic management journal, 20(5), 397-420.

Gulati, R., M. Gargiulo. (1999). Where do interorganizational networks come from? American Journal of Sociology, 104(5) 1439-1493.

Gulati, R., Sytch, M., \& Tatarynowicz, A. (2012). The rise and fall of small worlds: Exploring the dynamics of social structure. Organization Science, 23(2), 449-471.

Günter, H., Grote, G., \& Thees, O. (2006). Information technology in supply networks: Does it lead to better collaborative planning?. Journal of Enterprise Information Management, 19(5), 540-550. 
Hagedoorn, J. (2006). Understanding the cross-level embeddedness of interfirm partnership formation. Academy of management review, 31(3), 670-680.

Halinski, M., \& Duxbury, L. (2015). Getting what you want from your transorganizational partners: "Social-sharedness" and decision-making within a transorganizational system. Management Decision, 53(9), 2017-2035.

Harper, S. (1998). Leading organizational change in the $21^{\text {st }}$ century. Industrial Management, May-June, p. $25-31$.

Harris, L. C., Ogbonna, E., \& Goode, M. M. (2008). Intra-functional conflict: an investigation of antecedent factors in marketing functions. European Journal of Marketing, 42(3/4), 453476.

Harrison, R. (1970). Choosing the depth of organizational intervention. Journal of Applied Behavioral Science, 6, 182-202.

Harrison, J. S., \& John, C. H. S. (1996). Managing and partnering with external stakeholders. The Academy of Management Executive, 10(2), 46-60.

Hart, C. (1998). Doing a literature review: Releasing the social science research imagination. Sage.

Harvey, B., Schaefer, A. (2001). Managing Relationships with Environmental Stakeholders: A Study of U.K. Water and Electricity Utilities. Journal of Business Ethics, 30, p. 243-260.

Helms, W. S., \& Patterson, K. D. (2014). Eliciting acceptance for "illicit" organizations: The positive implications of stigma for MMA organizations. Academy of Management Journal, $57(5), 1453-1484$.

Hendriks, F., \& Tops, P. (2003). Local public management reforms in The Netherlands: fads, fashions and winds of change. Public Administration, 81(2), 301-323. 
Hennart, J. F., Kim, D. J., \& Zeng, M. (1998). The impact of joint venture status on the longevity of Japanese stakes in US manufacturing affiliates. Organization Science, 9(3), 382-395.

Hensmans, M. (2015). The Trojan horse mechanism and reciprocal sense-giving to urgent strategic change. Journal of Organizational Change Management, 28(6), 1038-1075.

Hoffman A. (1996). A strategic response to investor activism. Sloan Management Review 37(2): 51-65

Hollingshead, A. B. (1996). The rank-order effect in group decision making. Organizational Behavior and Human Decision Processes, 68(3), 181-193.

Hope, O. (2010). The politics of middle management sensemaking and sensegiving. Journal of Change Management, 10(2), 195-215.

Howard, M., Vidgen, R. \& Powell, P. (2003). Overcoming Stakeholder Barriers in the Automotive Industry: Building to Order with Extra-Organizational Systems. Journal of Information Technology, 18, p. 27-43.

Huber, G. P., \& Lewis, K. (2010). Cross-understanding: Implications for group cognition and performance. Academy of Management Review, 35(1), 6-26.

Hudson, B., Hardy, B., Henwood, M., \& Wistow, G. (1999). In pursuit of inter-agency collaboration in the public sector: What is the contribution of theory and research?. Public Management an International Journal of Research and Theory, 1(2), 235-260.

Huxham, C., \& Vangen, S. (2000). Leadership in the shaping and implementation of collaboration agendas: How things happen in a (not quite) joined-up world. Academy of Management journal, 43(6), 1159-1175.

Inkpen, A. C. (2000). Learning through joint ventures: a framework of knowledge acquisition. Journal of management studies, 37(7), 1019-1044. 
Islam, G. (2013). Finding a space for story: sensemaking, stories and epistemic impasse. Journal of Organizational Change Management, 26(1), 29-48.

Ivancevich, J. \& Matteson, M. (2002). Organizational behavior and management. Boston: McGraw-Hill.

Jackson, M. O., \& Rogers, B. W. (2007). Relating network structure to diffusion properties through stochastic dominance. The BE Journal of Theoretical Economics, 7(1).

Jaffe, D. T., Scott, C. D., \& Tobe, G. R. (1994). Rekindling commitment: How to revitalize yourself, your work, and your organization. Jossey-Bass.

Jamal, T. B., \& Getz, D. (1995). Collaboration theory and community tourism planning. Annals of tourism research, 22(1), 186-204.

Jawahar, I. and McLaughlin, G. (2001). Toward a descriptive stakeholder theory: an organizational life cycle approach. Academy of Management Review, 26 (3), pp. 397-414.

Jaworski, B. J., \& Kohli, A. K. (1996). Market orientation: review, refinement, and roadmap. Journal of Market-Focused Management, 1, 119-135.

Jespersen, P. K., Nielsen, L. L. M., \& Sognstrup, H. (2002). Professions, institutional dynamics, and new public management in the Danish hospital field. International Journal of Public Administration, 25(12), 1555-1574.

Jick, T. (1995). Accelerating change for competitive advantage. Organizational Dynamics, 14 (1), p. $77-82$.

Johnson, R. B., Onwuegbuzie, A. J., \& Turner, L. A. (2007). Toward a definition of mixed methods research. Journal of mixed methods research, 1(2), 112-133.

Johnson, G., and Scholes, K. (2002). Exploring Corporate Strategy, Sixth Edition. Harlow, England: Pearson Education. 
Jones, G. R. (2010). Organizational theory, design, and change. Upper Saddle River, NJ: Prentice Hall.

Jones, C., Hesterly, W. S., Fladmoe-Lindquist, K., \& Borgatti, S. P. (1998). Professional service constellations: How strategies and capabilities influence collaborative stability and change. Organization Science, 9(3), 396-410.

Jones, R. A., Jimmieson, N. L., \& Griffiths, A. (2005). The impact of organizational culture and reshaping capabilities on change implementation success: The mediating role of readiness for change. Journal of Management Studies, 42(2), 361-386.

Jones, T. and Wicks, A. (1999). Convergent stakeholder theory. Academy of Management Review, 24 (2), pp. 206-21.

Judson, S. (1991). Changing behavior in organizations: Minimizing resistance to change. Cambridge, MA: Basil Blackwell.

Kaghan, W. N., \& Lounsbury, M. (2006). Artifacts, articulation work and institutional residue. Artifacts and organizations: Beyond mere symbolism, 279-289.

Kahneman, D., \& Tversky, A. (1984). Choices, values, and frames. American psychologist, 39(4), 341.

Kale, P., Singh, H., \& Perlmutter, H. (2000). Learning and protection of proprietary assets in strategic alliances: Building relational capital. Strategic management journal, 21(3), 217237.

Kaler, J. (2002). Morality and strategy in stakeholder identification. Journal of Business Ethics, 39(1-2), 91-100.

Kameda, T., Hulbert, L., \& Tindale, R. S. (2002). Procedural and agenda effects on political decisions by small groups. In The social psychology of politics (pp. 215-240). Springer US. 
Kan, M. M., \& Parry, K. W. (2004). Identifying paradox: A grounded theory of leadership in overcoming resistance to change. The Leadership Quarterly, 15(4), 467-491.

Kanter, R. (1991). Transcending business boundaries: 12,000 world managers view change. MayJune, Harvard Business Review, p. 151 - 164.

Kanter, E., Stein, B. A., \& Jick, T. D. (1992). The challenge of organizational change: How companies experience it and leaders guide it. New York.

Keen, T. R. (2003). Creating effective and successful teams. Purdue University Press.

Kemelgor, B., Johnson, S., Srinivasan, S. (2000). Forces driving organizational change: A business school perspective. Journal of Education for Business, Jan.-Feb., p. 133 - 137.

Kerr, N. L., \& Tindale, R. S. (2004). Group performance and decision making. Annu. Rev. Psychol., 55, 623-655.

Key, S. (1999). Toward a new theory of the firm: a critique of stakeholder 'theory'. Management Decision, Vol. 37 No. 4, pp. 317-36.

Kezar, A. (2013). Understanding sensemaking/sensegiving in transformational change processes from the bottom up. Higher Education, 65(6), 761-780.

Kim, T.-Y., H. Oh, A. Swaminathan. (2006). Framing interorganizational network change: A network inertia perspective. Academy of Management Review, 31(3) 704-720.

Kippenberger, T. (1998). Planned change: Kurt Lewin's legacy. Antidote, The, 3(4), 10-12.

Knight, G., \& Greenberg, J. (2002). Promotionalism and subpolitics: Nike and its labor critics. Management Communication Quarterly, 16: 541-570.

Knights, D., \& McCabe, D. (2002). A road less travelled: beyond managerialist, critical and processual approaches to total quality management. Journal of Organizational Change Management, 15(3), 235-254. 
Kochan, T. A., \& Rubinstein, S. A. (2000). Toward a stakeholder theory of the firm: The Saturn partnership. Organization science, 11(4), 367-386.

Koka, B. R., Madhavan, R., \& Prescott, J. E. (2006). The evolution of interfirm networks: Environmental effects on patterns of network change. Academy of Management Review, 31(3), 721-737.

Kossek, E. E., \& Lobel, S. A. (1996). Introduction. Transforming human resource systems to manage diversity-an introduction and orienting framework. Managing diversity. Human resource strategies for transforming the workplace, 1-19.

Kossek, E., Markel, K. S., \& McHugh, P. P. (2003). Increasing diversity as an HRM change strategy. Journal of Organizational Change Management, 16(3), 328-352.

Kotter, J. P. (1995). Leading change: Why transformation efforts fail. Harvard business review, 73(2), 59-67.

Kotter, J. P. (2008). A sense of urgency. Harvard Business Press.

Kotter, J. P., \& Schlesinger, L. A. (1979). Choosing strategies for change (pp. pp-106). Harvard Business Review.

Krueger, R. A., \& Casey, M. A. (2000). Focus groups. A practical guide for applied research, 3.

Kuhn, T. S. (1974). Second thoughts on paradigms. The structure of scientific theories, 2, 459482.

Kuipers, B. S., Higgs, M., Kickert, W., Tummers, L., Grandia, J., \& Van der Voet, J. (2014). The management of change in public organizations: A literature review. Public Administration, 92(1), 1-20.

Kyriakidou, O. (2011). Relational perspectives on the construction of meaning: A network model of change interpretation. Journal of Organizational Change Management, 24(5), 572-592. 
Lane, P.J. \& Lubatkin, M. (1998). Relative absorptive capacity and interorganizational learning. Strategic Management Journal, 19, 461-477.

Langley, A., \& Abdallah, C. (2011). Templates and turns in qualitative studies of strategy and management. Research methodology in strategy and management, 6, 201-235.

Langley, A. N. N., Smallman, C., Tsoukas, H., \& Van de Ven, A. H. (2013). Process studies of change in organization and management: unveiling temporality, activity, and flow. Academy of Management Journal, 56(1), 1-13.

Lewin, K. (1943). 'Psychological ecology'. In Cartwright, D. (Ed.), Field Theory in Social Science. London: Social Science Paperbacks.

Lewin, K. (1946a). Action research and minority problems. Journal of social issues, 2(4), 34-46.

Lewin, K. (1946b). Force field analysis. The 1973 Annual Handbook for Group Facilitators, 11113.

Lewin, K. (1947a). 'Frontiers in group dynamics'. In Cartwright, D. (Ed.), Resolving Social Conflict. London: Harper \& Row.

Lewin, K. (1947b). Frontiers in Group Dynamics II. Channels of group life; social planning and action research. Human relations, 1(2), 143-153.

Lewin, K. (1947c). Group decision and social change. Readings in social psychology, 3, 197-211.

Lewin, K. (1951). Field theory in social science: Selected theoretical papers. New York: Harper.

Lewis, M. (2002). Child and adolescent psychiatry. 3rd ed. Phiadelphia (PA): Lippincott.

Logan, J. R., Stults, B. J., \& Farley, R. (2004). Segregation of minorities in the metropolis: Two decades of change. Demography, 41(1), 1-22.

Lounsbury, M., \& Crumley, E. T. (2007). New practice creation: An institutional perspective on innovation. Organization studies, 28(7), 993-1012. 
Lounsbury, M., \& Glynn, M. A. (2001). Cultural entrepreneurship: Stories, legitimacy, and the acquisition of resources. Strategic management journal, 22(6-7), 545-564.

Lunenburg, F. C. (2010). Forces for and resistance to organizational change. In National Forum of educational administration and supervision journal (Vol. 27, No. 4).

Luo, Y. (2005). How important are shared perceptions of procedural justice in cooperative alliances?. Academy of Management Journal, 48(4), 695-709.

Mainardes, E., Alves, H. \& Raposo, M. (2011). Stakeholder theory: issues to resolve. Management Decision, 49 (2), p. $226-252$.

Maitlis, S., \& Lawrence, T. B. (2007). Triggers and enablers of sensegiving in organizations. Academy of management Journal, 50(1), 57-84.

Maitlis, S., \& Sonenshein, S. (2010). Sensemaking in crisis and change: Inspiration and insights from Weick (1988). Journal of Management Studies, 47(3), 551-580.

Mariotti, F., \& Delbridge, R. (2012). Overcoming network overload and redundancy in interorganizational networks: The roles of potential and latent ties. Organization Science, 23(2), 511-528.

Marmenout, K. (2010). Employee sensemaking in mergers: How deal characteristics shape employee attitudes. The Journal of Applied Behavioral Science, 46(3), 329-359.

Marshak, R. J. (2002). Changing the language of change: How new contexts and concepts are challenging the ways we think and talk about organizational change. Strategic Change, $11(5), 279-286$.

Mason, M. (2010). Sample Size and Saturation in PhD Studies Using Qualitative Interviews. Qualitative Social Research, 11(3). Retrieved from http://www.qualitativeresearch.net/index.php/fqs/article/view/1428/3027 
Mangham, I. L. (1980). THE LIMITS OF PLANNED ORGANIZATIONAL CHANGE. Organisation development in Europe, 1, 359.

Mattox, K. (2001). The World Trade Center Attack Disaster preparedness: health care is ready, but is the bureaucracy?. Critical Care, 5(6), 323.

McCarthy, J. D., \& Zald, M. N. (2001). The enduring vitality of the resource mobilization theory of social movements. In Handbook of sociological theory (pp. 533-565). Springer US.

McGrath, J. \& Kelly, J. (1996). Time and human interaction: Toward a social psychology of time. New York: Guilford.

Mennecke, B., Roche, E., Bray, D., Konsynski, B., Lester, J., Rowe, M., \& Townsend, A. (2007, December). Second Life and other virtual worlds: A roadmap for research. In 28th International Conference on Information Systems (ICIS).

Mesquita, L. F., Anand, J., \& Brush, T. H. (2008). Comparing the resource-based and relational views: knowledge transfer and spillover in vertical alliances. Strategic Management Journal, 29(9), 913-941.

Mesquita, L. F., \& Lazzarini, S. G. (2008). Horizontal and vertical relationships in developing economies: Implications for SMEs' access to global markets. Academy of Management Journal, 51(2), 359-380.

Miles, M. B., \& Huberman, A. M. (1994). Qualitative data analysis: An expanded sourcebook. Sage.

Mitchell, R., Agle, B., Wood, D. (1997). Toward a Theory of Stakeholder Identification and Salience: Defining the Principle of Who and What Really Counts. Academy of Management Review, 22 (4), p. 853-886. 
Mizruchi, M. S. (1989). Similarity of political behavior among large American corporations. American Journal of Sociology, 401-424.

Mohr, L. (1982). Explaining organizational behavior. San Francisco: Jossey-Bass.

Monge, P. R., \& Contractor, N. S. (2003). Theories of communication networks. Oxford University Press, USA.

Moorman, C., \& Miner, A. S. (1998). The convergence of planning and execution: improvisation in new product development. the Journal of Marketing, 1-20.

Morrison, I. (1998). The second curve: Managing the velocity of change. Strategic Leadership, Jan.-Feb., p. 7 - 11.

Morse, J., Noerager Stern, P., Corbin, J., Bowers, B., Charmaz, K., and Clarke, A. (2009). Developing grounded theory. Walnut Creek, CA: Left Coast Press, Inc.

Morgan, G., \& Sturdy, A. (2000). Beyond organizational change. Palgrave Macmillan.

Morrison, E. W., \& Milliken, F. J. (2000). Organizational silence: A barrier to change and development in a pluralistic world. Academy of Management Review, 25(4), 706-725.

Mosakowski, E., \& Earley, P. C. (2000). A selective review of time assumptions in strategy research. Academy of Management Review, 25(4), 796-812.

Mulvale, G., Abelson, J., \& Goering, P. (2007). Mental health service delivery in Ontario, Canada: how do policy legacies shape prospects for reform?. HEALTH ECONOMICS POLICY AND LAW, 2(4), 363.

Myers, M. (2009).Qualitative Research in Business \& Management. SAGE.

Myers, D. G. (2012). Self-Serving bias. This Will Make You Smarter: New Scientific Concepts to Improve. 
Nadler, D. A., \& Tushman, M. L. (1989). Leadership for organizational change. Large-scale organizational change, 100, 119.

Nadler, D. \& Tushman, M. (1990). Beyond the charismatic leader: Leadership and organizational change. California Management Review, p. 77 - 97.

$\mathrm{Ng}$, K., Hase, S. (2008). Grounded suggestions for doing a grounded theory business research. Electronic Journal of Business Research Methods, 6 (2), p. p. 155-170.

Noor, K. (2008). Case Study: A Strategic research Methodology. American Journal of Applied Sciences, 5 (11), p. 1602-1604.

Oliver, A. L., \& Ebers, M. (1998). Networking network studies: an analysis of conceptual configurations in the study of interorganizational relationships. Organization studies, 19(4), 549-583.

Ong, B. K. (2012). Grounded Theory Method (GTM) and the Abductive Research Strategy (ARS): a critical analysis of their differences. International Journal of Social Research Methodology, 15(5), 417-432.

Orlikowski, W. J. (1996). Improvising organizational transformation over time: A situated change perspective. Information systems research, 7(1), 63-92.

Organisation for Economic Co-operation and Development. (1986). Competition Policy and Joint Ventures, Paris: OECD.

Oshry, B. (2007). Seeing systems: Unlocking the mysteries of organizational life. Berrett-Koehler Publishers.

Painter, J. (1991). Regulation theory and local government 1. Local Government Studies, 17(6), $23-44$. 
Papazoglou, M. P., Traverso, P., Dustdar, S., \& Leymann, F. (2008). Service-oriented computing: a research roadmap. International Journal of Cooperative Information Systems, 17(02), 223-255.

Parent, M. M., \& Deephouse, D. L. (2007). A case study of stakeholder identification and prioritization by managers. Journal of Business Ethics, 75(1), 1-23.

Patton, M. Q. (2002). Two Decades of Developments in Qualitative Inquiry A Personal, Experiential Perspective. Qualitative Social Work, 1(3), 261-283.

Pellé-Culpin, I. (1998). Du paradoxe de la diffusion d'information environnementale par les entreprises européennes (Doctoral dissertation).

Perlesz, A. \& Lindsay, J. (2003). Methodological triangulation in researching families: Making sense of dissonant data. International Journal of Social Research Methodology, 6 (1), p. $25-40$.

Pesqueux, Y. and Damak-Ayadi, S. (2005). Stakeholder theory in perspective. Corporate Governance. 5 (2), pp. 5-22.

Peteraf, M. A. (1993). The cornerstones of competitive advantage: A resource-based view. Strategic management journal, 14(3), 179-191.

Pettigrew, A. M. (1979). On studying organizational cultures. Administrative science quarterly, $570-581$.

Pettigrew, A., Woodman, R. \& Cameron, K. (2001). Studying organizational change and development challenges for future research. Academy of Management Journal, 44 (4), p. $697-713$.

Pfeffer J. (1981). Power in Organizations. Pitman: Marshfield, MA. 
Pfeffer, J., \& Veiga, J. F. (1999). Putting people first for organizational success. The Academy of Management Executive, 13(2), 37-48.

Phene, A., \& Tallman, S. (2014). Knowledge spillovers and alliance formation. Journal of Management Studies, 51(7), 1058-1090.

Phillips, R. (2003). Stakeholder legitimacy. Business Ethics Quarterly, 13(1), 25-41.

Piderit, S. K. (2000). Rethinking resistance and recognizing ambivalence: A multidimensional view of attitudes toward an organizational change. Academy of management review, 25(4), 783-794.

Pollitt, C., \& Bouckaert, G. (2003). Evaluating public management reforms: an international perspective. Evaluation in Public-Sector Reform. Concepts and Practice in International Perspective. Cheltenham, 12-35.

Poole, M., Van de Ven, A., Dooley, K., \& Holmes, M. (2000). Organizational change and innovation processes: Theory and methods for research. New York: Oxford University Press.

Porras, J. \& Hoffer, S. (1996). Common behavior changes in successful organization development efforts. Journal of Applied Behavioral Science, 22 (4), p. 477 - 494.

Porras, J. I., \& Silvers, R. C. (1991). Organization development and transformation. Annual review of Psychology, 42(1), 51-78.

Porter, M. E., \& Kramer, M. R. (2006). Strategy and society. Harvard business review, 84(12), 78-92.

Post, J. E., \& Altma, B. W. (1994). Managing the environmental change process: barriers and opportunities. Journal of Organizational Change Management, 7(4), 64-81. 
Post, J. \& Andrew, P. (1982). Case Research in Corporation and Society Studies. In L.E. Preston (ed.) Research in Corporate Social Performance Policy: A Research Annual, (p. 1 - 34), Greenwich, CT: Jai Press Inc.

Post, J., Preston, L. \& Sachs, S. (2002). Redefining The Corporation: Stakeholder Management and Organizational Wealth, Stanford University Press, Standford.

Powell, W. W., White, D. R., Koput, K. W., \& Owen-Smith, J. (2005). Network dynamics and field evolution: The growth of interorganizational collaboration in the life sciences1. American journal of sociology, 110(4), 1132-1205.

Rainey, H.G. 1997. Understanding and Managing Public Organizations, 2nd edn. San Francisco, CA: Jossey-Bass.

Reay, T., Golden-Biddle, K., \& Germann, K. (2006). Legitimizing a new role: Small wins and microprocesses of change. Academy of Management Journal, 49(5), 977-998.

Reay, T. and Hinings, S.R. (2005). The recomposition of an organizational field: Healthcare in Alberta. Organization Studies, 26(3), 351-384.

Reichard, C. (2003). Local public management reforms in Germany. Public Administration, 81(2), 345-363.

Reissner, S. C. (2011). Patterns of stories of organisational change. Journal of Organizational Change Management, 24(5), 593-609.

Rescher, N. (1996). Process metaphysics: An introduction to process philosophy. Suny Press.

Rico, R., Sanchez-Manzanares, M., Gil, F., and Gibson, C.B. (2008). Team coordination processes: A team knowledge-based approach. Academy of Management Review, 33(1): $163-185$. 
Riesenmy, K. (2010). Physician sensemaking and readiness for electronic medical records. The Learning Organization, 17(2), 163-177.

Rivera, M. T., S. B. Soderstrom, B. Uzzi. (2010). Dynamics of dyads in social networks: Assortative, relational, and proximity mechanisms. Annual Review of Sociology, 36 91115.

Roberts, N. (2001). Coping with Wicked Problems: The Case of Afghanistan. In Learning from International Public Management Reform, vol. 11, part B, edited by Lawrence Jones, James Guthrie, and Peter Steane, 353 - 75. New York: JAI Press.

Roberts, J. M. (2004). Alliances, coalitions and partnerships: Building collaborative organizations. New Society Publishers.

Rogers, E. (2004). Diffusion of innovations, 5th edn. New York: Free Press.

Romanelli, E. \& Tushman, M. (1994). Organizational transformation as punctuated equilibrium: an empirical test. Acad. Manage. J. 37:1141.66

Roome, N., \& Wijen, F. (2006). Stakeholder power and organizational learning in corporate environmental management. Organization Studies, 27(2), 235-263.

Rossi, J. (2006). Organizational change on the rise. $T \& D, 60$ (1), p. $15-15$.

Rossotti, C. O. (2005). Many unhappy returns: One man's quest to turn around the most unpopular organization in America. Harvard Business Press.

Rowley, T. J. (1997). Moving beyond dyadic ties: A network theory of stakeholder influences. Academy of management Review, 22(4), 887-910.

Rowley, T. I., \& Moldoveanu, M. (2003). When will stakeholder groups act? An interest-and identity-based model of stakeholder group mobilization. Academy of Management Review, 28(2), 204-219. 
Rouleau, L., \& Balogun, J. (2011). Middle managers, strategic sensemaking, and discursive competence. Journal of Management Studies, 48(5), 953-983.

Sabatier, P. A. (1998). The advocacy coalition framework: revisions and relevance for Europe. Journal of European public policy, 5(1), 98-130.

Scandura, T. A., \& Williams, E. A. (2000). Research methodology in management: Current practices, trends, and implications for future research. Academy of Management journal, 43(6), 1248-1264.

Schein, E. H. (1990). Organizational culture (Vol. 45, No. 2, p. 109). American Psychological Association.

Schein, E. H. (1996). Kurt Lewin's change theory in the field and in the classroom: Notes toward a model of managed learning. Systems Practice, 9(1), 27-47.

Schein, E. H. (2002). Models and tools for stability and change in human systems. Reflections, $4(2), 34-46$.

Senge, P. (2000). Lessons for change leaders. Leader to Leader, John Wiley \& Sons Inc., Fall, p. $21-37$.

Shah, S. K., \& Corley, K. G. (2006). Building Better Theory by Bridging the QuantitativeQualitative Divide. Journal of Management Studies, 43(8), 1821-1835.

Shoemaker, P. J., Eichholz, M., Kim, E., \& Wrigley, B. (2001). Individual and routine forces in gatekeeping. Journalism \& mass communication quarterly, 78(2), 233-246.

Siggelkow, N. (2007). Persuasion with case studies. Academy of Management Journal, 50(1), 2024. 
Sillince, J.A.A., Harindranath, G., and Harvey, C.E. (2001). Getting acceptance that radically new working practices are required: Institutionalization of arguments about change within a healthcare organization. Human Relations, 54(11), 1421-1454.

Sink, D. W. (1991). Transorganizational development in urban policy coalitions. Human relations, 44(11), 1179-1195.

Singleton, R., Straits, B. (2010). Approaches to Social Research (5 $5^{\text {th }}$ Ed.). New York City, NY: Oxford University Press.

Smith, A. (2012). 'Monday will never be the same again': the transformation of employment and work in a public-private partnership. Work, Employment \& Society, 26(1), 95-110.

Smollan, R. (2014). The emotional dimensions of metaphors of change. Journal of Managerial Psychology, 29(7), 794-807.

Snijders, T. A. (2011). Multilevel analysis (pp. 879-882). Springer Berlin Heidelberg.

Solomon, J. (1987). Social influences on the construction of pupils' understanding of science.

Sonenshein, S. (2010). We're Changing-Or are we? untangling the role of progressive, regressive, and stability narratives during strategic change implementation. Academy of Management Journal, 53(3), 477-512.

Sonnenwald, D. H. (2003). The conceptual organization: an emergent organizational form for collaborative R\&D. Science and Public Policy, 30(4), 261-272.

Spar, D. L., \& La Mure, L. T. (2003). The power of activism: Assessing the impact of NGOs on global business. California Management Review, 45(3): 78-101.

Starik, M. (1994). Essay by Mark Starik. Business \& Society. 33 (1), pp. 89-95. 
Stasser, G., \& Stewart, D. (1992). Discovery of hidden profiles by decision-making groups: Solving a problem versus making a judgment. Journal of personality and social psychology, 63(3), 426.

Stasser G, Titus W. 1985. Pooling of unshared information in group decision making: biased information sampling during discussion. Journal of Personal Social Psychology, 48:146778.

Steadman, H. J., Deane, M. W., Borum, R., \& Morrissey, J. P. (2000). Comparing outcomes of major models of police responses to mental health emergencies. Psychiatric Services, 51(5), 645-649.

Stensaker, I., Falkenberg, J., \& Grønhaug, K. (2008). Implementation activities and organizational sensemaking. The Journal of Applied Behavioral Science.

Stensaker, I. G., \& Meyer, C. B. (2011). Change experience and employee reactions: developing capabilities for change. Personnel review, 41(1), 106-124.

Sterner, R. W., Small, G. E. \& Hood, J. M. (2011). The Conservation of Mass. Nature Education Knowledge 3(10):20

Stoney, C. and Winstanley, D. (2001), "Stakeholding: confusion or Utopia? Mapping the conceptual terrain", Journal of Management Studies, Vol. 38 No. 5, pp. 603-26.

Suchman MC. (1995). Managing legitimacy: strategic and institutional approaches. Academy of Management Journal 20: 571-610.

Tajfel, H., \& Turner, J. C. (1979). An integrative theory of intergroup conflict. The social psychology of intergroup relations, 33(47), 74. 
Teulier, R., \& Rouleau, L. (2013). Middle managers' sensemaking and interorganizational change initiation: Translation spaces and editing practices. Journal of Change Management, 13(3), 308-337.

Thomas, D. A., \& Ely, R. J. (1996). Making differences matter: A new paradigm for managing diversity. Harvard business review, 74(5), 79.

Thompson, J. R., \& Sanders, R. P. (Eds.). (1997). Transforming government: Lessons from the reinvention laboratories. Jossey-Bass Incorporated Pub.

Thurmond, V. (2001). The point of triangulation. Journal of Nursing Scholarship, 33(3), 254-256.

Tilley, C. (1978). From mobilization to revolution. New York: Random House.

Tindale, R. S., \& Kameda, T. (2000). "Social 'sharedness"' as a unifying theme for information processing in groups. Group Processes \& Intergroup Relations, 3(2), 123-140.

Tindale RS, Smith CM, Thomas LS, Filkins J, Sheffey S. 1996. Shared representations and asymmetric social influence processes in small groups. In Understanding Group Behavior: Consensual Action by Small Groups, ed. E Witte, JH David, 1:81-103. Mahwah, NJ: Erlbaum.

Todnem By, R. (2005). Organisational change management: A critical review. Journal of Change Management, 5(4), 369-380.

Tsoukas, H. (2005). Complex knowledge: Studies in organizational epistemology. Oxford: Oxford University Press.

Tsoukas, H. \& Chia, R. (2002). On organizational becoming: Rethinking organizational change. Organization Science, 13(5), 567-582.

Tushman, M. L., Reilly, O., \& Charles III, A. (1996). Organizations: Managing Evolutionary. California management review, 38, 4. 
Tversky, A., \& Kahneman, D. (1992). Advances in prospect theory: Cumulative representation of uncertainty. Journal of Risk and uncertainty, 5(4), 297-323.

Uzzi, B. (1996). The sources and consequences of embeddedness for the economic performance of organizations: The network effect. American sociological review, 674-698.

Uzzi, B., \& Gillespie, J. J. (2002). Knowledge spillover in corporate financing networks: Embeddedness and the firm's debt performance. Strategic Management Journal, 23(7), 595-618.

Vaara, E., \& Monin, P. (2010). A recursive perspective on discursive legitimation and organizational action in mergers and acquisitions. Organization Science, 21(1), 3-22.

Van de Ven, A. H., \& Huber, G. P. (1990). Longitudinal field research methods for studying processes of organizational change. Organization science, 1(3), 213-219.

Van de Ven, A.G., and Poole, M.S. (1995). Explaining Development and change in organizations. Academy of Management Review, 20(3), 510-540.

Van de Ven, A. H., \& Poole, M. S. (2005). Alternative approaches for studying organizational change. Organization Studies, 26(9), 1377-1404.

Van Dyck, C., Frese, M., Baer, M., \& Sonnentag, S. (2005). Organizational error management culture and its impact on performance: a two-study replication. Journal of Applied Psychology, 90(6), 1228.

Vann, J. L. (2004). Resistance to change and the language of public organizations: A look at "clashing grammars" in large-scale information technology projects. Public Organization Review, 4(1), 47-73.

Vermesan, O., Friess, P., Guillemin, P., Gusmeroli, S., Sundmaeker, H., Bassi, A., ... \& Doody, P. (2011). Internet of things strategic research roadmap. O. Vermesan, P. Friess, P. Guillemin, 
S. Gusmeroli, H. Sundmaeker, A. Bassi, et al., Internet of Things: Global Technological and Societal Trends, 9-52.

Vermeulen, F., \& Barkema, H. (2001). Learning through acquisitions. Academy of Management journal, 44(3), 457-476.

Vollman, T. 1996. The transformation imperative. Boston, MA: Harvard Business School Press.

Wanous, J. P., Reichers, A. E., \& Austin, J. T. (2000). Cynicism about organizational change measurement, antecedents, and correlates. Group \& Organization Management, 25(2), $132-153$.

Warren, D., Jarvis, A., LeBlanc, L., Gravel, J., CTAS National Working Group. (2008). Revisions to the Canadian Triage and Acuity Scale Paediatric Guidelines (PaedCTAS). Canadian Journal of Emergency Medicine, 10 (3), p. 224 - 232.

Weber, E. P., \& Khademian, A. M. (2008). Wicked problems, knowledge challenges, and collaborative capacity builders in network settings. Public Administration Review, 68(2), 334-349.

Weick, K. (1979). The social psychology of organizing, 2nd edn. Reading, MA: Addison-Wesley.

Weick, K. E. (1993). The collapse of sensemaking in organizations: The Mann Gulch disaster. Administrative science quarterly, 628-652.

Weick, K. E. (1995). Sensemaking in organizations (Vol. 3). Sage.

Weick, K. E., \& Quinn, R. E. (1999). Organizational change and development. Annual review of psychology, 50(1), 361-386.

Weick, K. E., Sutcliffe, K. M., \& Obstfeld, D. (2005). Organizing and the process of sensemaking. Organization science, 16(4), 409-421. 
Westley, F., \& Vredenburg, H. (1991). Strategic bridging: The collaboration between environmentalists and business in the marketing of green products. The Journal of applied behavioral science, 27(1), 65-90.

Whelan, K. (1997). Change catalysts - sources of individual change in the context of organizational change. Dissertation Abstracts International, (UMI No. 9725149).

Whelan-Berry, K., Gordon, J. \& Hinings, C. (2003). The relative effect of change drivers in large scale organizational change: An empirical study. Research in Organizational Change and Development, 14, p. $99-146$.

Whetten, D. (2005). On the meaning of organizational in organizational studies: The case of organizational identity. Journal of Management Inquiry.

Wilson, J. Q. (2000). Bureaucracy: What government agencies do and why they do it. Basic Books.

Winn, M., Keller, L. (2001) A Modeling Methodology for Multiobjective Multistakeholder Decisions - Implications for Research. Journal of Management Inquiry, 10, p. 166-181.

Womack, J. P., \& Jones, D. T. (1996). Lean thinking: Banish waste and create wealth in your organization. Rawson Associates, New York.

Wood, D., Harms, P., \& Vazire, S. (2010). Perceiver effects as projective tests: what your perceptions of others say about you. Journal of personality and social psychology, 99(1), 174.

Worley, C. G., \& Parker, S. B. (2011). Building multi-stakeholder sustainability networks: The Cuyahoga valley initiative. Organizing for sustainable effectiveness, 1, 187-214.

Yin, R. (2003). Case study research: Design and methods. Sage.

Yin, R. K. (2009). Case study research: Design and methods (Vol. 5). Sage. 
Zander, A. (1950). Resistance to change - Its analysis and prevention. Advanced Management, 4 (5), p. $9-11$.

Zimmerman, M. A., \& Zeitz, G. J. (2002). Beyond survival: Achieving new venture growth by building legitimacy. Academy of Management Review, 27(3), 414-431. 


\section{APPENDIX A: TIME ONE INTERVIEW SCRIPT}

\section{Preamble}

Hi , This is Michael Halinski from the Sprott School of Business, Carleton University. I am calling for the interview we scheduled. Is now still an ok time for the interview? Reschedule if necessary or proceed.

Thank you for volunteering to take part in this brief interview. Before we begin I would like to take a moment to tell you a bit about this interview. You have been identified as someone who can provide us with insight into (1) the process of how persons with mental illness (PMI) are dealt with by police organizations and hospitals, and (2) the challenges of improving this process. This interview will take approximately 30-40 minutes of your time. I will ask you questions designed to help us gain a deeper understanding about your experiences regarding this process, challenges that may exist in changing this process and ways to improve this process.

The information that you give us will help us to identify aspects of the patient process that can be improved to benefit the key stakeholders involved in this process: Peel Regional Police, Brampton Civic Hospital, the families of those with mental illness, the individual themselves and the community. The results of these interviews will be the foundation for recommendations to improve the process.

Do you have any questions before we begin the interview?

\section{Try to answer any questions the interviewee asks.}

Thanks again for agreeing to take part in this interview. The interview today will be recorded to support the analysis of the data. Audio files will be stored on a password protected PC and will be deleted once the data has been coded and analyzed. Names and other identifying information will not be recorded or shared. Are you happy to go ahead?

If they are unhappy, try to identify and calm their concerns. If you cannot, proceed without recording and take detailed notes.

\section{Section 1: Background}

The first couple of questions give us some background information on you. This information will give us the context to help us interpret the data.

1a) What organization do you work for? 
1b) What is your role at this organization? (Get enough information to determine their position) Position

1c) How long have you been in this role? Years

1d) When did you first become involved with the PMI process?

1e) How are you involved with the PMI process? (Prompt: Describe your interactions with PMIs)

1f) How often would you say that you deal with the PMI process? (Prompt: Hourly, Daily, Weekly, Monthly) How often

\section{Section 2: PMI Process}

The majority of police organizations and hospitals in Ontario have been affected by the closure of mental health hospitals and the subsequent increase in the number of calls to the police involving persons with mental illness. Police and hospitals have been working together to manage this situation. Currently there are a set of steps that are undertaken when police and health care services are asked to manage "call" dealing with a person with mental illness - we refer to this set of steps as the PMI process. The next set of questions were designed to give us a greater understanding of how this process works from your perspective.

I would like you to walk me through the steps of the PMI process as you have experienced it (e.g. from your point of view).

2a) What starts the process? Is there any paper generated by this step in the process? How much time does this stage of the process typically take? How do you know when this stage is done?

2b) What happens next?

2c) And then what?

(Repeat questions until they say that there are no more steps).

Thank you very much for describing the PMI process to me. Now, let me describe the process back to you as I understand it from your description. (Describe process).

2d) Is there anything I have missed?

2e) From your perspective, what parts of this process work well the way they are?

2f) What parts of this process need to be changed? Why these? 
$2 \mathrm{~g}$ ) What changes would you personally make to the PMI process to make it more effective?

\section{Section 3: Multiple Stakeholders}

In academic circles, a stakeholder is defined as a person or organization with an interest or concern in something. In other words, a stakeholder is someone who contributes to the success or failure of an organization or a process. In this study, we are looking at people who have an interest in and perhaps contribute to the success or failure of the PMI process. In your perspective,

3a) Who do you think are the main stakeholders in the PMI process? (Top 5)

3b) What is the role of each stakeholder in the PMI process?

For each stakeholder identified,

3c) Why is $\mathrm{X}$ involved in the PMI process to begin with?

3d) What interest does $X$ have in the PMI process? In other words, why is the PMI process important to $\mathrm{X}$ ?

3e) What value does $X$ add to the PMI process? (i.e., what would happen if they were not involved?)

3f) How is $X$ important to the success of the PMI process?

$3 \mathrm{~g}$ ) How is the PMI process related to the success of $\mathrm{X}$ in doing its job/meeting its mandate?

\section{Section 4: Classifying Stakeholders Using Existing Theory}

Stakeholder theory suggests there are three ways to classify stakeholders: stakeholders with power, stakeholders with legitimacy and stakeholders with urgency. In this section of the interview I will define each of these types of stakeholders and then ask you to help me classify the key stakeholders in the PMI process (police, hospital and PMI) using these ideas.

\section{Power}

Let's begin with Power. A stakeholder who has power can get other stakeholders to do what they want, even if this may not be what they want to do. If we look at the relationship between teachers and students, teachers would be the stakeholders with power over the students. In your opinion,

4a) How is power displayed in the PMI process?

4b) How much power does the hospital have in the PMI process? How is it displayed? 
4c) How much power do the police have power the PMI process? How is it displayed?

4d) How much power does the social service agency have in the PMI process? How is it displayed?

4e) Who holds the most power of these three - or is it evenly distributed between the three groups?

4f) Are there any other stakeholders that I have not identified who also has power with respect to the PMI process? If yes, could you please describe this group and tell me what power they have in the PMI process?

\section{Legitimacy}

Next we have legitimacy. A legitimate stakeholder is someone (a group or individual) that people think should be involved with the PMI process. For example in the case of teachers and students, a teacher could be considered a legitimate stakeholder to their students as both students and teacher would agree that a teacher is required for students to learn. In your opinion,

$4 \mathrm{~g})$ How is legitimacy displayed in the PMI process?

4h) Why does someone or an organization participate in the PMI process? In other words, what makes it socially acceptable or okay for someone to be involved in the PMI process? (prompt: mental health mandate)

4i) At this point in time, the PMI process involves the police, health care personnel, people with mental illness and the judicial system. In your opinion, are all these groups legitimate stakeholders in the PMI process? Why or why not?

4j) By definition, legitimate stakeholders behave in a way that supports the PMI process (e.g. teachers behave in a way to support learning). Which stakeholders in the PMI process behave in a manner that supports this process? What do they do to support the process?

4k) Are there any behaviors from stakeholders that can be perceived as unsupportive of the PMI process? What are they? Who engages in such behaviours?

\section{Urgency}

And lastly, we have urgency. The idea of urgency suggests that the relationship between stakeholders is impacted by time, such that urgent stakeholders conduct activities that are time sensitive. So, in the case of students, the idea of studying is time sensitive. Students who do not study enough before their exam may receive a bad grade. As such, the students grade in a midterm 
is an indication to the teacher of how time sensitive the students regarded studying BEFORE they wrote the exam and whether or not they could be considered an urgent stakeholder. In your opinion,

4k) What actions (or steps) in the PMI process would you consider time sensitive?

41) Which stakeholder would suffer the most difficulty if these actions or steps are not performed efficiently?

$4 \mathrm{~m})$ Are there any delays in the PMI process? How do these delays affect the different stakeholders?

4n) Who is most negatively impacted by these delays?

\section{Section 5: Lean - Customer Value and Waste}

Lean is a workplace philosophy to make processes more efficient. Traditionally thought of as a manufacturing tool kit to reduce costs in the automotive industry, Lean has been introduced to the service industry, implemented in the Public Sector, healthcare and police organizations and provided substantial gains.

Lean is premised on increasing customer value and decreasing waste. In Lean terminology, the customer is the individual who benefits from the product or service and value is regarded as to what provides a better product/service to the customer (i.e., what makes them happy). For the next set of questions, I would like you to identify the ways each stakeholder benefit and the ways each stakeholder have drawbacks based on how the current process is managed.

You Identified Stakeholder $\boldsymbol{A}$ as a stakeholder in the PMI process.

5a) How do they benefit from the PMI process?

5b) What drawbacks do they encounter because of the way the PMI process is currently organized? (i.e., sources of waste)

(Repeat for each stakeholder)

\section{Section 6: Change Management}

Change management is a major part of this research project. Kurt Lewin, a very famous change researcher, suggested that successful change requires three steps: unfreezing 
(i.e., creating drivers for change and removing barriers to change), changing and refreezing (i.e., putting practices in place to ensure process doesn't revert back to old methods). The next set of questions deal with the first stage of change.

From your perspective,

6a) Does the PMI process need to change?

6b) What are the top 5 drivers of change?

6c) What are the top 5 barriers of change?

6d) What parts of the PMI process need to change? Why these parts?

For each of the changes they identified ask:

6e) Which stakeholder or stakeholders should be in charge of changing X? Why should they be in charge of this change?

\section{Section 7: Wrap Up}

Thank you very much for taking part in this interview.

Is there anything important that I missed? Do you have any questions that you wanted to ask me?

Would you be willing to be interviewed again in 12 months? If yes, what is the best way to reach you?

Email:

Phone:

I look forward to speaking with you then. 


\section{APPENDIX B: TIME TWO INTERVIEW SCRIPT}

\section{Preamble}

Hi , This is Michael Halinski from the Sprott School of Business, Carleton University. I am calling for the interview we scheduled. Is now still an ok time for the interview? Reschedule if necessary or proceed.

Thank you for your willingness to speak with me again regarding this important issue (i.e., managing individuals with mental illness in the community who lose insight). Before we begin I would like to take a moment to tell you a bit about this interview. The last time we spoke, roughly 1.5 years ago, you provided us with your perspective on the process of how persons with mental illness (PMI) are dealt with by police and hospitals - which we refer to as the PMI process - and the challenges of improving this process. Since then, police, hospital and CMHA management have made a series of changes to the PMI process. The purpose of this interview is to gain your perspective on these changes. This interview will take approximately 30-40 minutes of your time. I will ask you questions designed to help us gain a deeper understanding about your experiences regarding the PMI process, and changes that you perceive may or may not have taken place.

The information that you give us will help us better understand how to implement change in complex systems such as the one relating to the PMI process.

Do you have any questions before we begin the interview?

\section{Try to answer any questions the interviewee asks.}

Thanks again for agreeing to take part in this interview. The interview today will be recorded to support the analysis of the data. Audio files will be stored on a password protected PC and will be deleted once the data has been coded and analyzed. Names and other identifying information will not be recorded or shared. Are you happy to go ahead?

If they are unhappy, try to identify and calm their concerns. If you cannot, proceed without recording and take detailed notes.

\section{Section 1: Background}

The first couple of questions give us some background information on you. This information will give us the context to help us interpret the data.

1a) What organization do you work for? 
1b) What is your role at this organization? (Get enough information to determine their position) Position

1c) How long have you been in this role? Years

1d) How were you involved with the PMI process over the past year? (Prompt: Describe your interactions with PMIs)

1e) How often would you say that you dealt with the PMI process over the past year? (Prompt: Hourly, Daily, Weekly, Monthly) How often

\section{Section 2: PMI Process}

The PMI process refers to the set of steps that are taken by police and hospitals when individuals with mental illness are brought involuntarily to the hospital by the police to the time when police are allowed to leave the hospital. Over the past year, police, hospital and CMHA management have attempted to implement a series of changes to the PMI process. In your opinion,

2a) Do you think the process has changed over the past year?

2b) What has changed in the process changed over the past year? Why do you say this? What have you observed?

2c) How have these changes impacted you and your organization over the past year? Why do you say this?

2d) How have these changes impacted the Stakeholder $X$ and $Y$ over the past year? Why do you say this?

2e) What benefits have you or your organization realized from these changes? Why do you say this?

2f) What benefits have Stakeholder $X$ and $Y$ realized from these changes? Why do you say this?

$2 \mathrm{~g}$ ) What drawbacks have you or your organization experienced as a result of these changes? Why do you say this?

2h) What drawbacks have Stakeholder $X$ and $Y$ experienced as a result of these changes? Why do you say this?

2i) What has stayed the same in this process over the past year? Why do you say this?

$2 \mathrm{j}$ ) Overall, on a scale from $1-5$, where 5 is very positive, 1 is very negative and 3 is neutral, how would you evaluate the changes? Why do you say this?

$2 \mathrm{k}$ ) What still needs to change in the process? Why do you say this? 


\section{Section 3: TS Stakeholders}

In our last round of interviews, respondents identified the police, hospital and CMHA as the main parties who may participate in the PMI process, which is of course in addition to the actual PMI.

3a) What is the role of each stakeholder in the PMI process?

\section{Section 4: TS Stakeholder Relationships}

Theory suggests that the interactions of these groups are influenced by perceptions of power, legitimacy and urgency. In this section of the interview I will define each of these attributes and then ask you to help me determine how much power, legitimacy and urgency each group has in the PMI process.

\section{Power}

Let's begin with Power. A stakeholder who has power can get other stakeholders to do what they want, even if this may not be what they want to do. If we look at the relationship between teachers and students, teachers would be the stakeholders with power over the students. In your opinion,

4a) How is power displayed in the PMI process?

4b) How much power does the hospital have in the PMI process? How is it displayed?

4c) How much power do the police have power the PMI process? How is it displayed?

4d) How much power does the social service agency have in the PMI process? How is it displayed?

4e) Recalling that some changes have occurred in the PMI process in the past year, how have these changes in the PMI process affected how power is displayed in the PMI process over the past year? Why do you say this?

\section{Legitimacy}

Next we have legitimacy. A legitimate stakeholder is someone (a group or individual) that people think should be involved with the PMI process. For example in the case of teachers and 
students, a teacher could be considered a legitimate stakeholder to their students as both students and teacher would agree that a teacher is required for students to learn. In your opinion,

4f) How is legitimacy displayed in the PMI process?

4g) Why does someone or an organization participate in the PMI process? In other words, what makes it socially acceptable or okay for someone to be involved in the PMI process? (prompt: mental health mandate)

4h) By definition, legitimate stakeholders behave in a way that supports the PMI process (e.g. teachers behave in a way to support learning). Which stakeholders in the PMI process behave in a manner that supports this process? What do they do to support the process?

4i) Are there any behaviors from Stakeholder $X$ that can be perceived as unsupportive of the PMI process? What are they?

$4 \mathrm{j}$ ) Recalling that some changes have occurred in the PMI process in the past year, how have these changes in the PMI process affected what you view is a socially acceptable reason for participating in the PMI process over the past year? Why do you say this?

\section{Urgency}

And lastly, we have urgency. The idea of urgency suggests that the relationship between stakeholders is impacted by time, such that urgent stakeholders conduct activities that are time sensitive. So, in the case of students, the idea of studying is time sensitive. Students who do not study enough before their exam may receive a bad grade. As such, the students grade in a midterm is an indication to the teacher of how time sensitive the students regarded studying BEFORE they wrote the exam and whether or not they could be considered an urgent stakeholder. In your opinion,

4j) What actions (or steps) in the PMI process would you consider time sensitive?

4k) Which stakeholder would suffer the most difficulty if these actions or steps are not performed efficiently?

41) Are there any delays in the PMI process? How do these delays affect the different stakeholders?

$4 \mathrm{~m})$ Who is most negatively impacted by these delays? 
$4 n)$ Recalling that some changes have occurred in the PMI process in the past year, how have these changes in the PMI process affected what you consider time sensitive in the PMI process over the past year? Why do you say this?

\section{Section 5: Wrap Up}

Thank you very much for taking part in this interview.

Is there anything important that I missed? Do you have any questions that you wanted to ask me? 
APPENDIX C: FULL PANEL DATA DISPLAYS

\begin{tabular}{|c|c|c|c|}
\hline & $\begin{array}{l}\text { Perception } \\
\text { mentioned }\end{array}$ & $\begin{array}{l}\text { Hospital } \\
(\mathrm{n}=\mathbf{2 0})\end{array}$ & $\begin{array}{c}\text { Police } \\
(\mathbf{n}=\mathbf{2 0})\end{array}$ \\
\hline \multicolumn{4}{|l|}{$\begin{array}{l}\text { Perceived Power Displayed by } \\
\text { Hospital }\end{array}$} \\
\hline \multirow{2}{*}{ Doctor decides PMI's treatment } & $\begin{array}{l}\text { Said T1 } \\
\text { not T2 }\end{array}$ & $\begin{array}{c}15 \\
(75 \%)\end{array}$ & $\begin{array}{c}15 \\
(75 \%)\end{array}$ \\
\hline & $\begin{array}{l}\text { Said T2 } \\
\text { not T1 }\end{array}$ & $\begin{array}{c}4 \\
(20 \%)\end{array}$ & $\begin{array}{c}3 \\
(15 \%)\end{array}$ \\
\hline \multirow{2}{*}{$\begin{array}{r}\text { Hospital staff decides when police } \\
\text { officers can leave }\end{array}$} & $\begin{array}{l}\text { Said T1 } \\
\text { not T2 }\end{array}$ & $\begin{array}{c}6 \\
(30 \%)\end{array}$ & $\begin{array}{c}9 \\
(45 \%)\end{array}$ \\
\hline & $\begin{array}{c}\text { Said T2 } \\
\text { not T1 }\end{array}$ & $\begin{array}{c}4 \\
(20 \%)\end{array}$ & $\begin{array}{c}4 \\
(20 \%)\end{array}$ \\
\hline \multirow{2}{*}{$\begin{array}{r}\text { Doctor decides whether PMI is } \\
\text { formed }\end{array}$} & $\begin{array}{l}\text { Said T1 } \\
\text { not T2 }\end{array}$ & $\begin{array}{c}3 \\
(15 \%)\end{array}$ & $\begin{array}{c}1 \\
(5 \%)\end{array}$ \\
\hline & $\begin{array}{l}\text { Said T2 } \\
\text { not T1 }\end{array}$ & $\begin{array}{c}4 \\
(20 \%) \\
\end{array}$ & $\begin{array}{c}10 \\
(50 \%)\end{array}$ \\
\hline \multicolumn{4}{|l|}{$\begin{array}{l}\text { Perceived Power Displayed by } \\
\text { Police }\end{array}$} \\
\hline \multirow{2}{*}{$\begin{array}{r}\text { Police presence influences PMI } \\
\text { process flow }\end{array}$} & $\begin{array}{c}\text { Said T1 } \\
\text { not T2 }\end{array}$ & $\begin{array}{c}1 \\
(5 \%)\end{array}$ & $\begin{array}{c}0 \\
(0 \%) \\
\end{array}$ \\
\hline & $\begin{array}{l}\text { Said T2 } \\
\text { not T1 }\end{array}$ & $\begin{array}{c}5 \\
(25 \%)\end{array}$ & $\begin{array}{c}0 \\
(0 \%)\end{array}$ \\
\hline \multicolumn{4}{|l|}{$\begin{array}{l}\text { Perceived Power Displayed by } \\
\text { Social Service Agency }\end{array}$} \\
\hline & $\begin{array}{l}\text { Said T1 } \\
\text { not T2 }\end{array}$ & $\begin{array}{c}0 \\
(0 \%)\end{array}$ & $\begin{array}{c}0 \\
(0 \%)\end{array}$ \\
\hline & $\begin{array}{l}\text { Said T2 } \\
\text { not T1 }\end{array}$ & $\begin{array}{c}0 \\
(0 \%)\end{array}$ & $\begin{array}{c}0 \\
(0 \%)\end{array}$ \\
\hline
\end{tabular}

Note. $\mathrm{T} 1=$ Time $1 . \mathrm{T} 2=$ Time 2. Shaded cells identify changes in perceptions over time.

Table C-1: Perceptions of Power within the TS Over Time: Panel Data 


\begin{tabular}{|c|c|c|c|}
\hline & $\begin{array}{l}\text { Perception } \\
\text { mentioned }\end{array}$ & $\begin{array}{l}\text { Hospital } \\
(\mathrm{n}=\mathbf{2 0})\end{array}$ & $\begin{array}{l}\text { Police } \\
(\mathbf{n}=\mathbf{2 0})\end{array}$ \\
\hline \multicolumn{4}{|l|}{$\begin{array}{l}\text { Perceptions of Legitimate Police } \\
\text { Actions }\end{array}$} \\
\hline \multirow{2}{*}{$\begin{array}{r}\text { Police apprehend PMI under } \\
\text { Mental Health Act }\end{array}$} & $\begin{array}{c}\text { Said T1 } \\
\text { not T2 }\end{array}$ & $\begin{array}{c}6 \\
(30 \%)\end{array}$ & $\begin{array}{c}0 \\
(0 \%)\end{array}$ \\
\hline & $\begin{array}{l}\text { Said T2 } \\
\text { not T1 }\end{array}$ & $\begin{array}{c}1 \\
(5 \%)\end{array}$ & $\begin{array}{c}1 \\
(5 \%)\end{array}$ \\
\hline \multirow{2}{*}{ Police keep community safe } & $\begin{array}{l}\text { Said T1 } \\
\text { not T2 }\end{array}$ & $\begin{array}{c}1 \\
(5 \%)\end{array}$ & $\begin{array}{c}2 \\
(10 \%)\end{array}$ \\
\hline & $\begin{array}{l}\text { Said T2 } \\
\text { not T1 }\end{array}$ & $\begin{array}{c}0 \\
(0 \%)\end{array}$ & $\begin{array}{c}0 \\
(0 \%)\end{array}$ \\
\hline \multirow{2}{*}{$\begin{array}{r}\text { Police provide background } \\
\text { information about PMI to doctors }\end{array}$} & $\begin{array}{l}\text { Said T1 } \\
\text { not T2 }\end{array}$ & $\begin{array}{c}0 \\
(0 \%)\end{array}$ & $\begin{array}{c}3 \\
(15 \%)\end{array}$ \\
\hline & $\begin{array}{l}\text { Said T2 } \\
\text { not T1 }\end{array}$ & $\begin{array}{c}1 \\
(5 \%) \\
\end{array}$ & $\begin{array}{c}2 \\
(10 \%)\end{array}$ \\
\hline \multirow{2}{*}{$\begin{array}{r}\text { Police provide background } \\
\text { information about PMI to doctors }\end{array}$} & $\begin{array}{l}\text { Said T1 } \\
\text { not T2 }\end{array}$ & $\begin{array}{c}0 \\
(0 \%)\end{array}$ & $\begin{array}{c}1 \\
(5 \%)\end{array}$ \\
\hline & $\begin{array}{l}\text { Said T2 } \\
\text { not T1 }\end{array}$ & $\begin{array}{c}0 \\
(0 \%)\end{array}$ & $\begin{array}{c}0 \\
(0 \%)\end{array}$ \\
\hline \multirow{2}{*}{$\begin{array}{r}\text { Police can maintain order in } \\
\text { Hospital }\end{array}$} & $\begin{array}{l}\text { Said T1 } \\
\text { not T2 }\end{array}$ & $\begin{array}{c}0 \\
(0 \%)\end{array}$ & $\begin{array}{c}0 \\
(0 \%)\end{array}$ \\
\hline & $\begin{array}{l}\text { Said T2 } \\
\text { not T1 }\end{array}$ & $\begin{array}{c}0 \\
(0 \%)\end{array}$ & $\begin{array}{c}0 \\
(0 \%)\end{array}$ \\
\hline \multicolumn{4}{|l|}{$\begin{array}{l}\text { Perceptions of Legitimate } \\
\text { Hospital Actions }\end{array}$} \\
\hline \multirow{2}{*}{ Healthcare workers assess PMIs } & $\begin{array}{l}\text { Said T1 } \\
\text { not T2 }\end{array}$ & $\begin{array}{c}7 \\
(35 \%) \\
\end{array}$ & $\begin{array}{c}2 \\
(10 \%) \\
\end{array}$ \\
\hline & $\begin{array}{l}\text { Said T2 } \\
\text { not T1 }\end{array}$ & $\begin{array}{c}6 \\
(30 \%)\end{array}$ & $\begin{array}{c}1 \\
(5 \%)\end{array}$ \\
\hline \multirow{2}{*}{ Healthcare workers treat PMIs } & $\begin{array}{l}\text { Said T1 } \\
\text { not T2 }\end{array}$ & $\begin{array}{c}2 \\
(10 \%)\end{array}$ & $\begin{array}{c}3 \\
(15 \%) \\
\end{array}$ \\
\hline & $\begin{array}{l}\text { Said T2 } \\
\text { not T1 }\end{array}$ & $\begin{array}{c}0 \\
(0 \%)\end{array}$ & $\begin{array}{c}1 \\
(5 \%)\end{array}$ \\
\hline \multirow{2}{*}{ Hospital can discipline PMI } & $\begin{array}{l}\text { Said T1 } \\
\text { not T2 }\end{array}$ & $\begin{array}{c}2 \\
(10 \%)\end{array}$ & $\begin{array}{c}0 \\
(0 \%)\end{array}$ \\
\hline & $\begin{array}{l}\text { Said T2 } \\
\text { not T1 }\end{array}$ & $\begin{array}{c}0 \\
(0 \%)\end{array}$ & $\begin{array}{c}0 \\
(0 \%)\end{array}$ \\
\hline \multirow{2}{*}{$\begin{array}{r}\text { Other Hospital legitimacy (i.e. } \\
\text { healthcare policy, people seek out } \\
\text { hospitals) }\end{array}$} & $\begin{array}{c}\text { Said T1 } \\
\text { not T2 }\end{array}$ & $\begin{array}{c}1 \\
(5 \%)\end{array}$ & $\begin{array}{c}0 \\
(0 \%)\end{array}$ \\
\hline & $\begin{array}{c}\text { Said T2 } \\
\text { not T1 }\end{array}$ & $\begin{array}{c}0 \\
(0 \%)\end{array}$ & $\begin{array}{c}0 \\
(0 \%)\end{array}$ \\
\hline
\end{tabular}




\begin{tabular}{|c|c|c|c|}
\hline $\begin{array}{l}\text { Perceptions of Legitimate Social } \\
\text { Service Agency Actions }\end{array}$ & & & \\
\hline \multirow{2}{*}{$\begin{array}{r}\text { Social Service Agency workers } \\
\text { initiate PMI process }\end{array}$} & $\begin{array}{l}\text { Said T1 } \\
\text { not T2 }\end{array}$ & $\begin{array}{c}0 \\
(0 \%)\end{array}$ & $\begin{array}{c}0 \\
(0 \%)\end{array}$ \\
\hline & $\begin{array}{l}\text { Said T2 } \\
\text { not T1 }\end{array}$ & $\begin{array}{c}0 \\
(0 \%)\end{array}$ & $\begin{array}{c}0 \\
(0 \%)\end{array}$ \\
\hline \multirow{2}{*}{$\begin{array}{l}\text { Social Service Agency worker } \\
\text { supports PMI in community }\end{array}$} & $\begin{array}{l}\text { Said T1 } \\
\text { not T2 }\end{array}$ & $\begin{array}{c}1 \\
(5 \%)\end{array}$ & $\begin{array}{c}0 \\
(0 \%)\end{array}$ \\
\hline & $\begin{array}{l}\text { Said T2 } \\
\text { not T1 }\end{array}$ & $\begin{array}{c}0 \\
(0 \%)\end{array}$ & $\begin{array}{c}0 \\
(0 \%)\end{array}$ \\
\hline \multirow{2}{*}{$\begin{array}{r}\text { Other Social Service Agency } \\
\text { legitimacy during PMI process }\end{array}$} & $\begin{array}{l}\text { Said T1 } \\
\text { not T2 }\end{array}$ & $\begin{array}{c}0 \\
(0 \%)\end{array}$ & $\begin{array}{c}0 \\
(0 \%)\end{array}$ \\
\hline & $\begin{array}{l}\text { Said T2 } \\
\text { not T1 }\end{array}$ & $\begin{array}{c}0 \\
(0 \%)\end{array}$ & $\begin{array}{c}0 \\
(0 \%)\end{array}$ \\
\hline \multirow{2}{*}{$\begin{array}{l}\text { Social workers provide PMI } \\
\text { information to Hospital and Police }\end{array}$} & $\begin{array}{l}\text { Said T1 } \\
\text { not T2 }\end{array}$ & $\begin{array}{c}0 \\
(0 \%)\end{array}$ & $\begin{array}{c}0 \\
(0 \%)\end{array}$ \\
\hline & $\begin{array}{l}\text { Said T2 } \\
\text { not T1 }\end{array}$ & $\begin{array}{c}0 \\
(0 \%)\end{array}$ & $\begin{array}{c}0 \\
(0 \%)\end{array}$ \\
\hline \multirow{2}{*}{$\begin{array}{r}\text { Social workers advocate for PMI } \\
\text { to Hospital and Police }\end{array}$} & $\begin{array}{l}\text { Said T1 } \\
\text { not T2 }\end{array}$ & $\begin{array}{c}0 \\
(0 \%)\end{array}$ & $\begin{array}{c}0 \\
(0 \%)\end{array}$ \\
\hline & $\begin{array}{l}\text { Said T2 } \\
\text { not T1 }\end{array}$ & $\begin{array}{c}0 \\
(0 \%)\end{array}$ & $\begin{array}{c}0 \\
(0 \%)\end{array}$ \\
\hline \multirow{2}{*}{$\begin{array}{r}\text { InSted workers connect PMIs to } \\
\text { community services }\end{array}$} & $\begin{array}{l}\text { Said T1 } \\
\text { not T2 }\end{array}$ & $\begin{array}{c}0 \\
(0 \%)\end{array}$ & $\begin{array}{c}0 \\
(0 \%)\end{array}$ \\
\hline & $\begin{array}{l}\text { Said T2 } \\
\text { not T1 }\end{array}$ & $\begin{array}{c}0 \\
(0 \%)\end{array}$ & $\begin{array}{c}0 \\
(0 \%)\end{array}$ \\
\hline
\end{tabular}

Note. $\mathrm{T} 1=\mathrm{Time} 1 . \mathrm{T} 2=$ Time 2 . Shaded cells identify changes in perceptions of legitimacy.

Table C-2: Perceptions of Legitimacy within the TS Over Time: Panel Data 


\begin{tabular}{|c|c|c|c|}
\hline & $\begin{array}{l}\text { Perception } \\
\text { mentioned }\end{array}$ & $\begin{array}{l}\text { Hospital } \\
(\mathrm{n}=\mathbf{2 0})\end{array}$ & $\begin{array}{c}\text { Police } \\
(n=20)\end{array}$ \\
\hline \multicolumn{4}{|l|}{$\begin{array}{l}\text { Perceptions of Urgent Hospital } \\
\text { Actions }\end{array}$} \\
\hline \multirow{2}{*}{$\begin{array}{l}\text { Physicians need to assess PMIs in } \\
\text { a timely manner }\end{array}$} & $\begin{array}{l}\text { Said T1 } \\
\text { not T2 }\end{array}$ & $\begin{array}{c}3 \\
(15 \%)\end{array}$ & $\begin{array}{c}0 \\
(0 \%)\end{array}$ \\
\hline & $\begin{array}{l}\text { Said T2 } \\
\text { not T1 }\end{array}$ & $\begin{array}{c}10 \\
(50 \%)\end{array}$ & $\begin{array}{c}10 \\
(50 \%)\end{array}$ \\
\hline \multirow{2}{*}{$\begin{array}{l}\text { Physicians discharge PMIs in a } \\
\text { timely manner }\end{array}$} & $\begin{array}{l}\text { Said T1 } \\
\text { not T2 }\end{array}$ & $\begin{array}{c}0 \\
(0 \%)\end{array}$ & $\begin{array}{c}0 \\
(0 \%)\end{array}$ \\
\hline & $\begin{array}{l}\text { Said T2 } \\
\text { not T1 }\end{array}$ & $\begin{array}{c}5 \\
(25 \%)\end{array}$ & $\begin{array}{c}0 \\
(0 \%)\end{array}$ \\
\hline \multicolumn{4}{|l|}{$\begin{array}{l}\text { Perceptions of Urgent Police } \\
\text { Actions }\end{array}$} \\
\hline \multirow{2}{*}{$\begin{array}{r}\text { Police transport PMI to Hospital in } \\
\text { a timely manner }\end{array}$} & $\begin{array}{l}\text { Said T1 } \\
\text { not T2 }\end{array}$ & $\begin{array}{c}5 \\
(25 \%) \\
\end{array}$ & $\begin{array}{c}12 \\
(60 \%) \\
\end{array}$ \\
\hline & $\begin{array}{l}\text { Said T2 } \\
\text { not T1 }\end{array}$ & $\begin{array}{c}1 \\
(5 \%) \\
\end{array}$ & $\begin{array}{c}1 \\
(5 \%) \\
\end{array}$ \\
\hline \multirow{2}{*}{$\begin{array}{r}\text { Police leaving Hospital in a timely } \\
\text { manner }\end{array}$} & $\begin{array}{l}\text { Said T1 } \\
\text { not T2 }\end{array}$ & $\begin{array}{c}16 \\
(80 \%)\end{array}$ & $\begin{array}{c}14 \\
(70 \%)\end{array}$ \\
\hline & $\begin{array}{l}\text { Said T2 } \\
\text { not T1 }\end{array}$ & $\begin{array}{c}0 \\
(0 \%)\end{array}$ & $\begin{array}{c}0 \\
(0 \%)\end{array}$ \\
\hline \multirow{2}{*}{$\begin{array}{l}\text { Other Hospital Urgency (i.e. } \\
\text { medicate PMI, discharge PMI) }\end{array}$} & $\begin{array}{l}\text { Said T1 } \\
\text { not T2 }\end{array}$ & $\begin{array}{c}1 \\
(5 \%)\end{array}$ & $\begin{array}{c}0 \\
(0 \%)\end{array}$ \\
\hline & $\begin{array}{l}\text { Said T2 } \\
\text { not T1 }\end{array}$ & $\begin{array}{c}0 \\
(0 \%)\end{array}$ & $\begin{array}{c}0 \\
(0 \%)\end{array}$ \\
\hline \multicolumn{4}{|l|}{$\begin{array}{l}\text { Perceptions of Urgent Social } \\
\text { Service Agency Actions }\end{array}$} \\
\hline \multirow{2}{*}{$\begin{array}{r}\text { Community support worker calls } \\
911 \text { to request police assistance } \\
\text { with high risk PMI }\end{array}$} & $\begin{array}{l}\text { Said T1 } \\
\text { not T2 }\end{array}$ & $\begin{array}{c}0 \\
(0 \%)\end{array}$ & $\begin{array}{c}0 \\
(0 \%)\end{array}$ \\
\hline & $\begin{array}{l}\text { Said T2 } \\
\text { not T1 }\end{array}$ & $\begin{array}{c}0 \\
(0 \%)\end{array}$ & $\begin{array}{c}0 \\
(0 \%)\end{array}$ \\
\hline \multirow{2}{*}{$\begin{array}{l}\text { Community support worker locates } \\
\text { PMI after Hospital discharge }\end{array}$} & $\begin{array}{l}\text { Said T1 } \\
\text { not T2 }\end{array}$ & $\begin{array}{c}0 \\
(0 \%)\end{array}$ & $\begin{array}{c}0 \\
(0 \%)\end{array}$ \\
\hline & $\begin{array}{l}\text { Said T2 } \\
\text { not T1 }\end{array}$ & $\begin{array}{c}0 \\
(0 \%)\end{array}$ & $\begin{array}{c}0 \\
(0 \%)\end{array}$ \\
\hline \multirow{2}{*}{$\begin{array}{l}\text { Community support worker } \\
\text { connects the PMI with community } \\
\text { services after Hospital discharge }\end{array}$} & $\begin{array}{c}\text { Said T1 } \\
\text { not T2 }\end{array}$ & $\begin{array}{c}0 \\
(0 \%)\end{array}$ & $\begin{array}{c}0 \\
(0 \%)\end{array}$ \\
\hline & $\begin{array}{c}\text { Said T2 } \\
\text { not T1 }\end{array}$ & $\begin{array}{c}0 \\
(0 \%)\end{array}$ & $\begin{array}{c}0 \\
(0 \%)\end{array}$ \\
\hline
\end{tabular}

Note. $\mathrm{T} 1=$ Time $1 . \mathrm{T} 2=$ Time 2 . Shaded cells identify changes in perceptions of urgency.

Table C-3: Perceptions of Urgency within the TS Over Time: Panel Data 


\section{APPENDIX D: DATA DISPLAYS FOR INTRA-ORGANIZATIONAL ASSESSMENTS}

Figure D-1: Perceptions of Power within the TS: TS Stakeholder Sample Pre-Change (Time 1)

\begin{tabular}{|c|c|c|c|c|c|c|c|c|c|c|c|c|}
\hline \multirow{3}{*}{$\begin{array}{l}\text { Perceptions of Power within the TS: TS } \\
\text { Stakeholder Sample Pre-Change (Time } \\
\text { 1) }\end{array}$} & \multicolumn{12}{|c|}{ INTER ORGANIZATIONAL ASSESSMENT } \\
\hline & \multicolumn{4}{|c|}{$\begin{array}{l}\text { Hospital } \\
(n=25)\end{array}$} & \multicolumn{4}{|c|}{$\begin{array}{l}\text { Police } \\
(n=25)\end{array}$} & \multicolumn{4}{|c|}{$\begin{array}{l}\text { Social Service } \\
\quad(\mathbf{n}=\mathbf{2 5})\end{array}$} \\
\hline & \multicolumn{2}{|c|}{ \# } & \multicolumn{2}{|c|}{$\%$} & \multicolumn{2}{|c|}{ \# } & \multicolumn{2}{|c|}{$\%$} & \multicolumn{2}{|c|}{ \# } & \multicolumn{2}{|c|}{$\%$} \\
\hline Perceived Power Displayed by Hospital & \multicolumn{2}{|c|}{24} & \multicolumn{2}{|c|}{96} & \multicolumn{2}{|c|}{24} & \multicolumn{2}{|c|}{96} & \multicolumn{2}{|c|}{22} & \multicolumn{2}{|c|}{88} \\
\hline Doctor decides PMI's treatment & \multicolumn{2}{|c|}{18} & \multicolumn{2}{|c|}{72} & \multicolumn{2}{|c|}{15} & \multicolumn{2}{|c|}{60} & \multicolumn{2}{|c|}{11} & \multicolumn{2}{|c|}{44} \\
\hline Hospital staff decides when police officers can leave & \multicolumn{2}{|c|}{9} & \multicolumn{2}{|c|}{36} & \multicolumn{2}{|c|}{13} & \multicolumn{2}{|c|}{52} & \multicolumn{2}{|c|}{8} & \multicolumn{2}{|c|}{32} \\
\hline Doctor decides whether PMI is formed & \multicolumn{2}{|c|}{6} & \multicolumn{2}{|c|}{24} & \multicolumn{2}{|c|}{4} & \multicolumn{2}{|c|}{16} & & & & 4 \\
\hline $\begin{array}{l}\text { Perceived Power Displayed by Social Service } \\
\text { Agency }\end{array}$ & & & & & & & & & & & & \\
\hline Social worker requests Police assistance & & & & & & & & & & 0 & & 0 \\
\hline Perceived Power Displayed by Police & & & & & & & & & & 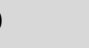 & & 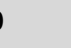 \\
\hline Police presence influences PMI process flow & & & & & & & & & & ) & & ) \\
\hline & & & & & A O & NIZA & NAI & SESSN & & & & \\
\hline & & & & & & & & & & $\begin{array}{l}\text { Justice } \\
\text { 11) }\end{array}$ & & Team \\
\hline & \# & $\%$ & \# & $\%$ & \# & $\%$ & $\#$ & $\%$ & $\#$ & $\%$ & \# & $\%$ \\
\hline Perceived Power Displayed by Hospital & 15 & 94 & 9 & 100 & 18 & 100 & 6 & 86 & 9 & 82 & 13 & 93 \\
\hline Doctor decides PMI's treatment & 12 & 75 & 6 & 67 & 12 & 67 & 3 & 43 & 3 & 27 & 8 & 57 \\
\hline Hospital staff decides when police officers can leave & 6 & 38 & 3 & 33 & 9 & 50 & 4 & 57 & 2 & 18 & 6 & 43 \\
\hline Doctor decides whether PMI is formed & 2 & 13 & 4 & 44 & 4 & 22 & 0 & 0 & 5 & 45 & 6 & 43 \\
\hline $\begin{array}{l}\text { Perceived Power Displayed by Social Service } \\
\text { Agency }\end{array}$ & 0 & 0 & 0 & 0 & 0 & 0 & 0 & 0 & 4 & 36 & 6 & 43 \\
\hline Social worker requests Police assistance & 0 & 0 & 0 & 0 & 0 & 0 & 0 & 0 & 4 & 36 & 6 & 43 \\
\hline
\end{tabular}




\begin{tabular}{|c|c|c|c|c|c|c|c|c|c|c|c|c|}
\hline Perceived Power Displayed by Police & 4 & 25 & 2 & 22 & 0 & 0 & 0 & 0 & 0 & 0 & 0 & 0 \\
\hline Police presence influences PMI process flow & 4 & 25 & 2 & 22 & 0 & 0 & 0 & 0 & 0 & 0 & 0 & 0 \\
\hline
\end{tabular}


Figure D-2: Perceptions of Power within the TS: TS Stakeholder Sample Post-Change (Time 2)

\begin{tabular}{|c|c|c|c|c|c|c|c|c|c|c|c|c|}
\hline \multirow{3}{*}{$\begin{array}{l}\text { Perceptions of Power in the TS: TS } \\
\text { Stakeholder Sample Post-Change (Time } \\
\text { 2) }\end{array}$} & \multicolumn{12}{|c|}{ INTER ORGANIZATIONAL ASSESSMENT } \\
\hline & \multicolumn{4}{|c|}{$\begin{array}{l}\text { Hospital } \\
(n=20)\end{array}$} & \multicolumn{4}{|c|}{$\begin{array}{l}\text { Police } \\
(\mathbf{n}=\mathbf{2 0})\end{array}$} & \multicolumn{4}{|c|}{$\begin{array}{l}\text { Social Service } \\
\qquad(\mathbf{n}=\mathbf{2 0})\end{array}$} \\
\hline & \multicolumn{2}{|c|}{ \# } & \multicolumn{2}{|c|}{$\%$} & \multicolumn{2}{|c|}{ \# } & \multicolumn{2}{|c|}{$\%$} & \multicolumn{2}{|c|}{ \# } & \multicolumn{2}{|c|}{$\%$} \\
\hline Perceived Power Displayed by Hospital & \multicolumn{2}{|c|}{14} & \multicolumn{2}{|c|}{70} & \multicolumn{2}{|c|}{20} & \multicolumn{2}{|c|}{100} & \multicolumn{2}{|c|}{19} & \multicolumn{2}{|c|}{95} \\
\hline Doctor decides whether PMI is formed & \multicolumn{2}{|c|}{7} & \multicolumn{2}{|c|}{35} & \multicolumn{2}{|c|}{13} & \multicolumn{2}{|c|}{65} & \multicolumn{2}{|c|}{17} & \multicolumn{2}{|c|}{85} \\
\hline Hospital staff decides when police officers can leave & \multicolumn{2}{|c|}{8} & \multicolumn{2}{|c|}{40} & \multicolumn{2}{|c|}{8} & \multicolumn{2}{|c|}{40} & \multicolumn{2}{|c|}{2} & \multicolumn{2}{|c|}{10} \\
\hline Doctor decides PMI's treatment & \multicolumn{2}{|c|}{7} & \multicolumn{2}{|c|}{35} & \multicolumn{2}{|c|}{3} & & & & & & \\
\hline Perceived Power Displayed by Police & & & & & & & & & & l & & \\
\hline Police presence influences PMI process flow & & & & & & & & & & & & \\
\hline $\begin{array}{l}\text { Perceived Power Displayed by Social Service } \\
\text { Agency }\end{array}$ & & & & & & & & & & ; & & \\
\hline Social worker requests Police assistance & & & & & & & & & & & & \\
\hline & & & & & A Ol & NIZA & NAL & SESSM & & & & \\
\hline & & & & & & & & & $\mathrm{MH}$ & $\begin{array}{l}\text { Justice } \\
\text { 9) }\end{array}$ & & 11) \\
\hline & $\#$ & $\%$ & \# & $\%$ & \# & $\%$ & \# & $\%$ & \# & $\%$ & \# & $\%$ \\
\hline Perceived Power Displayed by Hospital & 8 & 62 & 6 & 86 & 14 & 100 & 6 & 100 & 9 & 100 & 10 & 91 \\
\hline Doctor decides whether PMI is formed & 5 & 38 & 2 & 29 & 12 & 86 & 2 & 33 & 8 & 89 & 8 & 73 \\
\hline Hospital staff decides when police officers can leave & 4 & 31 & 5 & 71 & 3 & 21 & 5 & 83 & 2 & 22 & 0 & 0 \\
\hline Doctor decides PMI's treatment & 4 & 31 & 2 & 29 & 2 & 14 & 0 & 0 & 1 & 11 & 7 & 64 \\
\hline Perceived Power Displayed by Police & 6 & 46 & 1 & 14 & 0 & 0 & 0 & 0 & 1 & 11 & 6 & 55 \\
\hline Police presence influences PMI process flow & 6 & 46 & 1 & 14 & 0 & 0 & 0 & 0 & 1 & 11 & 6 & 55 \\
\hline $\begin{array}{l}\text { Perceived Power Displayed by Social Service } \\
\text { Agency }\end{array}$ & 0 & 0 & 0 & 0 & 0 & 0 & 0 & 0 & 3 & 33 & 2 & 18 \\
\hline Social worker requests Police assistance & 0 & 0 & 0 & 0 & 0 & 0 & 0 & 0 & 3 & 33 & 2 & 18 \\
\hline
\end{tabular}


Figure D-3: Perceptions of Legitimacy within the TS: TS Stakeholder Sample Pre-Change (Time 1)

\begin{tabular}{|c|c|c|c|c|c|c|c|c|c|c|c|c|}
\hline \multirow{3}{*}{$\begin{array}{c}\text { Perceptions of Legitimacy within the TS: } \\
\text { TS Stakeholder Sample Pre-Change } \\
\text { (Time 1) }\end{array}$} & \multicolumn{12}{|c|}{ INTER ORGANIZATIONAL ASSESSMENT } \\
\hline & \multicolumn{4}{|c|}{$\begin{array}{l}\text { Hospital } \\
(n=25)\end{array}$} & \multicolumn{4}{|c|}{$\begin{array}{l}\text { Police } \\
(n=25)\end{array}$} & \multicolumn{4}{|c|}{$\begin{array}{l}\text { Social Service } \\
\quad(n=25)\end{array}$} \\
\hline & \multicolumn{2}{|c|}{ \# } & \multicolumn{2}{|c|}{$\%$} & \multicolumn{2}{|c|}{ \# } & \multicolumn{2}{|c|}{$\%$} & \multicolumn{2}{|c|}{ \# } & \multicolumn{2}{|c|}{$\%$} \\
\hline Perceptions of Legitimate Police Actions & \multicolumn{2}{|c|}{24} & \multicolumn{2}{|c|}{96} & \multicolumn{2}{|c|}{25} & \multicolumn{2}{|c|}{100} & \multicolumn{2}{|c|}{22} & \multicolumn{2}{|c|}{88} \\
\hline Police apprehend PMIs under Mental Health Act & \multicolumn{2}{|c|}{14} & \multicolumn{2}{|c|}{56} & \multicolumn{2}{|c|}{24} & \multicolumn{2}{|c|}{96} & \multicolumn{2}{|c|}{12} & \multicolumn{2}{|c|}{48} \\
\hline Police keep community safe & \multicolumn{2}{|c|}{12} & \multicolumn{2}{|c|}{48} & \multicolumn{2}{|c|}{12} & \multicolumn{2}{|c|}{48} & \multicolumn{2}{|c|}{14} & \multicolumn{2}{|c|}{56} \\
\hline Police provide information to doctor & \multicolumn{2}{|c|}{7} & \multicolumn{2}{|c|}{28} & \multicolumn{2}{|c|}{8} & & & & & & \\
\hline Perceptions of Legitimate Hospital Actions & & & & & & & & & & & & \\
\hline Healthcare workers assess PMIs & & & & & & & & & & & & \\
\hline Healthcare workers treat PMIs & & & & & & & & & & & & \\
\hline $\begin{array}{l}\text { Perceptions of Legitimate Social Service Agency } \\
\text { Actions }\end{array}$ & & & & & & & & & & & & \\
\hline Social Service Agency workers initiate PMI process & & & & & & & & & & & & \\
\hline $\begin{array}{r}\text { Social Service Agency worker supports PMI in } \\
\text { community }\end{array}$ & & & & & & & & & & & & \\
\hline & & & & & A OF & NIZA & NAL & SESSM & & & & \\
\hline & & & & & & & & & MH & $\begin{array}{l}\text { Uustice } \\
11)\end{array}$ & & \\
\hline & \# & $\%$ & \# & $\%$ & \# & $\%$ & \# & $\%$ & \# & $\%$ & \# & $\%$ \\
\hline Perceptions of Legitimate Police Actions & 15 & 94 & 9 & 100 & 18 & 100 & 7 & 100 & 9 & 82 & 13 & 93 \\
\hline Police apprehend PMIs under Mental Health Act & 10 & 63 & 4 & 44 & 18 & 100 & 6 & 86 & 7 & 64 & 5 & 36 \\
\hline Police keep community safe & 6 & 38 & 6 & 67 & 10 & 56 & 2 & 29 & 7 & 64 & 7 & 50 \\
\hline Police provide information to doctor & 4 & 25 & 3 & 33 & 3 & 17 & 5 & 71 & 2 & 18 & 2 & 14 \\
\hline Perceptions of Legitimate Hospital Actions & 9 & 56 & 8 & 89 & 12 & 67 & 5 & 71 & 4 & 36 & 8 & 57 \\
\hline
\end{tabular}




\begin{tabular}{|c|c|c|c|c|c|c|c|c|c|c|c|c|}
\hline Healthcare workers assess PMIs & 8 & 50 & 5 & 56 & 11 & 61 & 4 & 57 & 4 & 36 & 4 & 29 \\
\hline Healthcare workers treat PMIs & 1 & 6 & 4 & 44 & 3 & 17 & 1 & 14 & 0 & 0 & 6 & 43 \\
\hline $\begin{array}{l}\text { Perceptions of Legitimate Social Service Agency } \\
\text { Actions }\end{array}$ & 3 & 19 & 3 & 33 & 0 & 0 & 0 & 0 & 8 & 73 & 10 & 71 \\
\hline Social Service Agency workers initiate PMI process & 0 & 0 & 0 & 0 & 0 & 0 & 0 & 0 & 6 & 55 & 8 & 57 \\
\hline $\begin{array}{r}\text { Social Service Agency worker supports PMI in } \\
\text { community }\end{array}$ & 3 & 19 & 3 & 33 & 0 & 0 & 0 & 0 & 7 & 63 & 3 & 21 \\
\hline
\end{tabular}


Figure D-4: Perceptions of Legitimacy within the TS: TS Stakeholder Sample Post-Change (Time 2)

\begin{tabular}{|c|c|c|c|c|c|c|c|c|c|c|c|c|}
\hline \multirow{3}{*}{$\begin{array}{c}\text { Perceptions of Legitimacy within the TS: } \\
\text { TS Stakeholder Sample Post-Change } \\
\text { (Time 2) }\end{array}$} & \multicolumn{12}{|c|}{ INTER ORGANIZATIONAL ASSESSMENT } \\
\hline & \multicolumn{4}{|c|}{$\begin{array}{l}\text { Hospital } \\
(n=20)\end{array}$} & \multicolumn{4}{|c|}{$\begin{array}{l}\text { Police } \\
(\mathbf{n}=\mathbf{2 0})\end{array}$} & \multicolumn{4}{|c|}{$\begin{array}{l}\text { Social Service } \\
\quad(n=20)\end{array}$} \\
\hline & \multicolumn{2}{|c|}{ \# } & \multicolumn{2}{|c|}{$\%$} & \multicolumn{2}{|c|}{ \# } & \multicolumn{2}{|c|}{$\%$} & \multicolumn{2}{|c|}{ \# } & \multicolumn{2}{|c|}{$\%$} \\
\hline Perceptions of Legitimate Police Actions & \multicolumn{2}{|c|}{12} & \multicolumn{2}{|c|}{60} & \multicolumn{2}{|c|}{20} & \multicolumn{2}{|c|}{100} & \multicolumn{2}{|c|}{17} & \multicolumn{2}{|c|}{85} \\
\hline Apprehend PMIs under mental health act & \multicolumn{2}{|c|}{12} & \multicolumn{2}{|c|}{60} & \multicolumn{2}{|c|}{20} & \multicolumn{2}{|c|}{100} & \multicolumn{2}{|c|}{17} & \multicolumn{2}{|c|}{85} \\
\hline Police keep community safe & \multicolumn{2}{|c|}{1} & \multicolumn{2}{|c|}{5} & \multicolumn{2}{|c|}{4} & \multicolumn{2}{|c|}{20} & \multicolumn{2}{|c|}{0} & \multicolumn{2}{|c|}{0} \\
\hline Police provide information to doctor & \multicolumn{2}{|c|}{0} & \multicolumn{2}{|c|}{0} & \multicolumn{2}{|c|}{0} & & & & & & 0 \\
\hline Perceptions of Legitimate Police Actions & & & & & & & & & & & & 0 \\
\hline Apprehend PMIs under mental health act & & & & & & & & & & & & 5 \\
\hline Police keep community safe & & & & & & & & & & & & 5 \\
\hline $\begin{array}{l}\text { Perceptions of Legitimate Social Service Agency } \\
\text { Actions }\end{array}$ & & & & & & & & & & & & 5 \\
\hline $\begin{array}{r}\text { Social workers provide PMI information to Hospital } \\
\text { and Police }\end{array}$ & & & & & & & & & & & & 0 \\
\hline Advocate for PMI to Hospital and Police & & & & & & & & & & 0 & & 0 \\
\hline InSted connects PMIs to community services & & & & & & & & & & & & 5 \\
\hline & & & & & A O & ANIZA & NAL & SESSM & & & & \\
\hline & & & & & & & & & MH & $\begin{array}{l}\text { Justice } \\
\text { 9) }\end{array}$ & & $\begin{array}{l}\text { Team } \\
11)\end{array}$ \\
\hline & \# & $\%$ & \# & $\%$ & \# & $\%$ & \# & $\%$ & \# & $\%$ & \# & $\%$ \\
\hline Perceptions of Legitimate Police Actions & 6 & 46 & 6 & 86 & 14 & 100 & 6 & 100 & 9 & 100 & 8 & 73 \\
\hline Apprehend PMIs under mental health act & 6 & 46 & 6 & 86 & 14 & 100 & 6 & 100 & 9 & 100 & 8 & 73 \\
\hline Police keep community safe & 1 & 8 & 0 & 0 & 4 & 28 & 0 & 0 & 0 & 0 & 0 & 0 \\
\hline Police provide information to doctor & 0 & 0 & 0 & 0 & 0 & 0 & 0 & 0 & 1 & 11 & 1 & 9 \\
\hline Perceptions of Legitimate Police Actions & 8 & 64 & 6 & 86 & 2 & 14 & 1 & 17 & 3 & 33 & 1 & 9 \\
\hline
\end{tabular}




\begin{tabular}{|c|c|c|c|c|c|c|c|c|c|c|c|c|}
\hline Apprehend PMIs under mental health act & 8 & 64 & 6 & 86 & 2 & 14 & 0 & 0 & 3 & 33 & 0 & 0 \\
\hline Police keep community safe & 0 & 0 & 0 & 0 & 2 & 14 & 1 & 17 & 2 & 22 & 1 & 9 \\
\hline $\begin{array}{l}\text { Perceptions of Legitimate Social Service Agency } \\
\text { Actions }\end{array}$ & 0 & 0 & 0 & 0 & 2 & 14 & 0 & 0 & 7 & 78 & 10 & 90 \\
\hline $\begin{array}{r}\text { Social workers provide PMI information to Hospital } \\
\text { and Police }\end{array}$ & 0 & 0 & 0 & 0 & 1 & 7 & 0 & 0 & 6 & 67 & 4 & 36 \\
\hline Advocate for PMI to Hospital and Police & 0 & 0 & 0 & 0 & 0 & 0 & 0 & 0 & 4 & 67 & 6 & 55 \\
\hline InSted connects PMIs to community services & 0 & 0 & 0 & 0 & 1 & 7 & 0 & 0 & 0 & 0 & 7 & 64 \\
\hline
\end{tabular}


Figure D-5: Perceptions of Urgency within the TS: TS Stakeholder Sample Pre-Change (Time 1)

\begin{tabular}{|c|c|c|c|c|c|c|c|c|c|c|c|c|}
\hline \multirow{3}{*}{$\begin{array}{c}\text { Perceptions of Urgency within the TS: } \\
\text { TS Stakeholder Sample Pre-Change } \\
\text { (Time 1) }\end{array}$} & \multicolumn{12}{|c|}{ INTER ORGANIZATIONAL ASSESSMENT } \\
\hline & \multicolumn{4}{|c|}{$\begin{array}{l}\text { Hospital } \\
(n=25)\end{array}$} & \multicolumn{4}{|c|}{$\begin{array}{l}\text { Police } \\
(n=25)\end{array}$} & \multicolumn{4}{|c|}{$\begin{array}{l}\text { Social Service } \\
\quad(n=25)\end{array}$} \\
\hline & \multicolumn{2}{|c|}{ \# } & \multicolumn{2}{|c|}{$\%$} & \multicolumn{2}{|c|}{ \# } & \multicolumn{2}{|c|}{$\%$} & \multicolumn{2}{|c|}{ \# } & \multicolumn{2}{|c|}{$\%$} \\
\hline Perceptions of Urgent Police Actions & \multicolumn{2}{|c|}{24} & \multicolumn{2}{|c|}{96} & \multicolumn{2}{|c|}{24} & \multicolumn{2}{|c|}{96} & \multicolumn{2}{|c|}{23} & \multicolumn{2}{|c|}{92} \\
\hline Police leave Hospital in a timely manner & \multicolumn{2}{|c|}{24} & \multicolumn{2}{|c|}{96} & \multicolumn{2}{|c|}{24} & \multicolumn{2}{|c|}{96} & \multicolumn{2}{|c|}{20} & \multicolumn{2}{|c|}{80} \\
\hline Police transport PMI to Hospital in a timely manner & \multicolumn{2}{|c|}{4} & \multicolumn{2}{|c|}{16} & \multicolumn{2}{|c|}{10} & \multicolumn{2}{|c|}{40} & \multicolumn{2}{|c|}{13} & \multicolumn{2}{|c|}{52} \\
\hline Perceptions of Urgent Hospital Actions & \multicolumn{2}{|c|}{9} & \multicolumn{2}{|c|}{36} & \multicolumn{2}{|c|}{1} & \multicolumn{2}{|c|}{4} & & & & \\
\hline Physicians need to assess PMIs in a timely manner & & & & & & & & & & & & \\
\hline $\begin{array}{l}\text { Perceptions of Urgent Social Service Agency } \\
\text { Actions }\end{array}$ & & & & & & & & & & & & \\
\hline $\begin{array}{r}\text { Community support worker calls } 911 \text { to request } \\
\text { police assistance with high risk PMI }\end{array}$ & & & & & & & & & & & & \\
\hline & & & & & A Ol & INIZA & NAL & ESSM & & & & \\
\hline & & & & & & & & & MH & $\begin{array}{l}\text { Justice } \\
\text { 11) }\end{array}$ & & 14) \\
\hline & \# & $\%$ & \# & $\%$ & \# & $\%$ & \# & $\%$ & \# & $\%$ & \# & $\%$ \\
\hline Perceptions of Urgent Police Actions & 15 & 94 & 9 & 100 & 17 & 95 & 7 & 100 & 11 & 100 & 12 & 86 \\
\hline Police leave Hospital in a timely manner & 15 & 94 & 9 & 100 & 17 & 95 & 7 & 100 & 11 & 100 & 9 & 64 \\
\hline Police transport PMI to Hospital in a timely manner & 2 & 12 & 2 & 22 & 8 & 44 & 2 & 29 & 4 & 36 & 9 & 64 \\
\hline Perceptions of Urgent Hospital Actions & 4 & 25 & 3 & 33 & 1 & 6 & 1 & 14 & 0 & 0 & 2 & 14 \\
\hline Physicians need to assess PMIs in a timely manner & 4 & 25 & 3 & 33 & 1 & 6 & 0 & 0 & 0 & 0 & 1 & 7 \\
\hline $\begin{array}{l}\text { Perceptions of Urgent Social Service Agency } \\
\text { Actions }\end{array}$ & 0 & 0 & 0 & 0 & 0 & 0 & 0 & 0 & 4 & 36 & 6 & 43 \\
\hline $\begin{array}{r}\text { Community support worker calls } 911 \text { to request } \\
\text { police assistance with high risk PMI }\end{array}$ & 0 & 0 & 0 & 0 & 0 & 0 & 0 & 0 & 4 & 36 & 4 & 29 \\
\hline
\end{tabular}


Figure D-6: Perceptions of Urgency within the TS: TS Stakeholder Sample Post-Change (Time 2)

\begin{tabular}{|c|c|c|c|c|c|c|c|c|c|c|c|c|}
\hline \multirow{3}{*}{$\begin{array}{c}\text { Perceptions of Urgency within the TS: } \\
\text { TS Stakeholder Sample Post-Change } \\
\text { (Time 2) }\end{array}$} & \multicolumn{12}{|c|}{ INTER ORGANIZATIONAL ASSESSMENT } \\
\hline & \multicolumn{4}{|c|}{$\begin{array}{l}\text { Hospital } \\
(n=20)\end{array}$} & \multicolumn{4}{|c|}{$\begin{array}{l}\text { Police } \\
(n=20)\end{array}$} & \multicolumn{4}{|c|}{$\begin{array}{l}\text { Social Service } \\
\quad(n=20)\end{array}$} \\
\hline & \multicolumn{2}{|c|}{ \# } & \multicolumn{2}{|c|}{$\%$} & \multicolumn{2}{|c|}{ \# } & \multicolumn{2}{|c|}{$\%$} & \multicolumn{2}{|c|}{ \# } & \multicolumn{2}{|c|}{$\%$} \\
\hline Perceptions of Urgent Hospital Actions & \multicolumn{2}{|c|}{16} & \multicolumn{2}{|c|}{80} & \multicolumn{2}{|c|}{11} & \multicolumn{2}{|c|}{55} & \multicolumn{2}{|c|}{15} & \multicolumn{2}{|c|}{75} \\
\hline Physicians need to assess PMIs in a timely manner & \multicolumn{2}{|c|}{16} & \multicolumn{2}{|c|}{80} & \multicolumn{2}{|c|}{11} & \multicolumn{2}{|c|}{55} & \multicolumn{2}{|c|}{10} & \multicolumn{2}{|c|}{50} \\
\hline Physicians discharge PMIs in a timely manner & \multicolumn{2}{|c|}{5} & \multicolumn{2}{|c|}{25} & \multicolumn{2}{|c|}{0} & \multicolumn{2}{|c|}{0} & \multicolumn{2}{|c|}{5} & \multicolumn{2}{|c|}{25} \\
\hline Perceptions of Urgent Police Actions & \multicolumn{2}{|c|}{6} & \multicolumn{2}{|c|}{30} & \multicolumn{2}{|c|}{12} & \multicolumn{2}{|c|}{60} & & & & \\
\hline Police transport PMI to Hospital in a timely manner & & & & & & & & & & & & \\
\hline Police leaving Hospital in a timely manner & & & & & & & & & & & & \\
\hline $\begin{array}{l}\text { Perceptions of Urgent Social Service Agency } \\
\text { Actions }\end{array}$ & & & & & & & & & & & & \\
\hline $\begin{array}{l}\text { Community support worker connects the PMI with } \\
\text { community services after Hospital discharge }\end{array}$ & & & & & & & & & & & & \\
\hline & & & & & A O & NIZA & NAL & ESSN & & & & \\
\hline & & & & & & & & & & $\begin{array}{l}\text { Uustice } \\
\text { 9) }\end{array}$ & & 11) \\
\hline & $\#$ & $\%$ & \# & $\%$ & \# & $\%$ & \# & $\%$ & \# & $\%$ & $\#$ & $\%$ \\
\hline Perceptions of Urgent Hospital Actions & 11 & 85 & 5 & 71 & 9 & 64 & 2 & 33 & 7 & 78 & 9 & 82 \\
\hline Physicians need to assess PMIs in a timely manner & 7 & 64 & 3 & 43 & 6 & 43 & 2 & 33 & 7 & 78 & 4 & 36 \\
\hline Physicians discharge PMIs in a timely manner & 6 & 46 & 1 & 14 & 6 & 43 & 1 & 17 & 3 & 33 & 4 & 36 \\
\hline Perceptions of Urgent Police Actions & 2 & 15 & 4 & 57 & 8 & 57 & 4 & 67 & 6 & 67 & 6 & 55 \\
\hline Police transport PMI to Hospital in a timely manner & 2 & 15 & 1 & 14 & 7 & 50 & 1 & 17 & 6 & 67 & 6 & 55 \\
\hline Police leaving Hospital in a timely manner & 1 & 8 & 4 & 57 & 2 & 14 & 4 & 67 & 2 & 22 & 0 & 0 \\
\hline
\end{tabular}




\begin{tabular}{|c|c|c|c|c|c|c|c|c|c|c|c|c|}
\hline $\begin{array}{l}\text { Perceptions of Urgent Social Service Agency } \\
\text { Actions }\end{array}$ & 0 & 0 & 0 & 0 & 0 & 0 & 0 & 0 & 2 & 22 & 2 & 18 \\
\hline $\begin{array}{l}\text { Community support worker connects the PMI with } \\
\text { community services after Hospital discharge }\end{array}$ & 0 & 0 & 0 & 0 & 0 & 0 & 0 & 0 & 2 & 22 & 2 & 18 \\
\hline
\end{tabular}


Figure D-7: Perceptions of Desired Changes in the TS: TS Stakeholder Sample Pre-Change (Time 1)

\begin{tabular}{|c|c|c|c|c|c|c|c|c|c|c|c|c|}
\hline \multirow{3}{*}{$\begin{array}{c}\text { Perceptions of Desired Changes in the } \\
\text { TS: TS Stakeholder Sample Pre-Change } \\
\text { (Time 1) }\end{array}$} & \multicolumn{12}{|c|}{ INTER ORGANIZATIONAL ASSESSMENT } \\
\hline & \multicolumn{4}{|c|}{$\begin{array}{l}\text { Hospital } \\
(n=25)\end{array}$} & \multicolumn{4}{|c|}{$\begin{array}{l}\text { Police } \\
(n=25)\end{array}$} & \multicolumn{4}{|c|}{$\begin{array}{l}\text { Social Service } \\
\quad(n=25)\end{array}$} \\
\hline & \multicolumn{2}{|c|}{ \# } & \multicolumn{2}{|c|}{$\%$} & \multicolumn{2}{|c|}{ \# } & \multicolumn{2}{|c|}{$\%$} & \multicolumn{2}{|c|}{ \# } & \multicolumn{2}{|c|}{$\%$} \\
\hline Desired Hospital Changes & \multicolumn{2}{|c|}{23} & \multicolumn{2}{|c|}{92} & \multicolumn{2}{|c|}{25} & \multicolumn{2}{|c|}{100} & \multicolumn{2}{|c|}{11} & \multicolumn{2}{|c|}{40} \\
\hline Shorter waiting time for PMI and Police & \multicolumn{2}{|c|}{20} & \multicolumn{2}{|c|}{80} & \multicolumn{2}{|c|}{25} & \multicolumn{2}{|c|}{100} & \multicolumn{2}{|c|}{10} & \multicolumn{2}{|c|}{40} \\
\hline Increased MHESU engagement & \multicolumn{2}{|c|}{21} & \multicolumn{2}{|c|}{84} & \multicolumn{2}{|c|}{7} & \multicolumn{2}{|c|}{28} & \multicolumn{2}{|c|}{5} & \multicolumn{2}{|c|}{20} \\
\hline Desired Changes at System Level & \multicolumn{2}{|c|}{4} & \multicolumn{2}{|c|}{16} & \multicolumn{2}{|c|}{7} & & & & & & \\
\hline $\begin{array}{r}\text { More effective communication between TS } \\
\text { stakeholders }\end{array}$ & & & & & & & & & & & & \\
\hline Desired Police Changes & & & & & & & & & & & & \\
\hline Training Police to apprehend PMIs appropriately & & & & & & & & & & & & \\
\hline Police complete EDP form & & & & & & & & & & & & \\
\hline & & & & & A Ol & NIZA & NAL & SESSI & & & & \\
\hline & & & & & & & & & $\mathrm{MH}$ & $\begin{array}{l}\text { Justice } \\
11)\end{array}$ & & $\begin{array}{l}\text { Team } \\
14)\end{array}$ \\
\hline & \# & $\%$ & \# & $\%$ & \# & $\%$ & $\#$ & $\%$ & $\#$ & $\%$ & \# & $\%$ \\
\hline Desired Hospital Changes & 14 & 88 & 8 & 89 & 18 & 100 & 6 & 86 & 5 & 45 & 6 & 43 \\
\hline Shorter waiting time for PMI and Police & 12 & 75 & 8 & 89 & 12 & 67 & 3 & 43 & 3 & 27 & 6 & 43 \\
\hline Increased MHESU engagement & 9 & 56 & 7 & 78 & 2 & 11 & 0 & 0 & 0 & 0 & 3 & 21 \\
\hline Desired Changes at System Level & 2 & 13 & 2 & 22 & 4 & 22 & 3 & 43 & 2 & 18 & 11 & 79 \\
\hline $\begin{array}{r}\text { More effective communication between TS } \\
\text { stakeholders } \\
\end{array}$ & 2 & 13 & 2 & 22 & 4 & 22 & 3 & 43 & 2 & 18 & 11 & 79 \\
\hline Desired Police Changes & 2 & 13 & 5 & 56 & 0 & 0 & 0 & 0 & 5 & 45 & 4 & 29 \\
\hline Training Police to apprehend PMIs appropriately & 0 & 0 & 0 & 0 & 0 & 0 & 0 & 0 & 5 & 45 & 4 & 29 \\
\hline Police complete EDP form & 2 & 13 & 5 & 56 & 0 & 0 & 0 & 0 & 1 & 9 & 0 & 0 \\
\hline
\end{tabular}


Figure D-8: Perceptions of What Has Changed in the TS: TS Stakeholder Sample Post-Change (Time 2)

\begin{tabular}{|c|c|c|c|c|c|c|c|c|c|c|c|c|}
\hline \multirow{3}{*}{$\begin{array}{c}\text { Perceptions of What Has Changed in the } \\
\text { TS: TS Stakeholder Sample Post-Change } \\
\text { (Time 2) }\end{array}$} & \multicolumn{12}{|c|}{ INTER ORGANIZATIONAL ASSESSMENT } \\
\hline & \multicolumn{4}{|c|}{$\begin{array}{l}\text { Hospital } \\
(n=20)\end{array}$} & \multicolumn{4}{|c|}{$\begin{array}{l}\text { Police } \\
(\mathbf{n}=\mathbf{2 0})\end{array}$} & \multicolumn{4}{|c|}{$\begin{array}{l}\text { Social Service } \\
\quad(\mathbf{n}=\mathbf{2 0})\end{array}$} \\
\hline & \multicolumn{2}{|c|}{$\#$} & \multicolumn{2}{|c|}{$\%$} & \multicolumn{2}{|c|}{ \# } & \multicolumn{2}{|c|}{$\%$} & \multicolumn{2}{|c|}{ \# } & \multicolumn{2}{|c|}{$\%$} \\
\hline Perceived System Level Changes & \multicolumn{2}{|c|}{12} & \multicolumn{2}{|c|}{60} & \multicolumn{2}{|c|}{13} & \multicolumn{2}{|c|}{65} & \multicolumn{2}{|c|}{12} & \multicolumn{2}{|c|}{60} \\
\hline Increased interorganizational communication & \multicolumn{2}{|c|}{12} & \multicolumn{2}{|c|}{60} & \multicolumn{2}{|c|}{13} & \multicolumn{2}{|c|}{65} & \multicolumn{2}{|c|}{12} & \multicolumn{2}{|c|}{60} \\
\hline Perceived Hospital Changes & \multicolumn{2}{|c|}{18} & \multicolumn{2}{|c|}{90} & \multicolumn{2}{|c|}{17} & \multicolumn{2}{|c|}{85} & \multicolumn{2}{|c|}{$\mathbf{0}$} & \multicolumn{2}{|c|}{$\mathbf{0}$} \\
\hline $\begin{array}{r}\text { ED created new process that expedites service for } \\
\text { PMIs who are brought in by Police }\end{array}$ & \multicolumn{2}{|c|}{18} & \multicolumn{2}{|c|}{90} & \multicolumn{2}{|c|}{17} & \multicolumn{2}{|c|}{85} & & & & \\
\hline $\begin{array}{r}\text { Hospital increased communication between ED and } \\
\text { MHESU }\end{array}$ & & & & & & & & & & & & \\
\hline Perceived Social Service Agency Changes & & & & & & & & & & & & \\
\hline $\begin{array}{r}\text { Social Service Agency created InSted program to } \\
\text { support PMI }\end{array}$ & & & & & & & & & & & & \\
\hline Perceived Police Changes & & & & & & & & & & & & \\
\hline Police mandate completion of EDP form & & & & & & & & & & & & \\
\hline $\begin{array}{r}\text { Police increased communication between frontline } \\
\text { officers and supervisors }\end{array}$ & & & & & & & & & & & & \\
\hline & & & & & $\mathrm{AOH}$ & NIZA & NAL & SESSM & & & & \\
\hline & & & & & & & & & MH & lustice & & \\
\hline & \# & $\%$ & \# & $\%$ & $\#$ & $\%$ & \# & $\%$ & $\#$ & $\%$ & \# & $\%$ \\
\hline Perceived System Level Changes & 6 & 46 & 6 & 86 & 8 & 57 & 5 & 83 & 3 & 33 & 7 & 64 \\
\hline Increased interorganizational communication & 6 & 46 & 6 & 86 & 8 & 57 & 5 & 83 & 3 & 33 & 7 & 64 \\
\hline Perceived Hospital Changes & 12 & 92 & 6 & 86 & 11 & 79 & 6 & 100 & 0 & 0 & 0 & 0 \\
\hline $\begin{array}{r}\text { ED created new process that expedites service for } \\
\text { PMIs who are brought in by Police }\end{array}$ & 12 & 92 & 6 & 86 & 11 & 79 & 6 & 100 & 0 & 0 & 0 & 0 \\
\hline
\end{tabular}




\begin{tabular}{|c|c|c|c|c|c|c|c|c|c|c|c|c|}
\hline $\begin{array}{r}\text { Hospital increased communication between ED and } \\
\text { MHESU }\end{array}$ & 1 & 8 & 3 & 43 & 3 & 21 & 1 & 17 & 0 & 0 & 0 & 0 \\
\hline Perceived Social Service Agency Changes & 1 & 8 & 3 & 43 & 0 & 0 & 0 & 0 & 7 & 78 & 9 & 82 \\
\hline $\begin{array}{r}\text { Social Service Agency created InSted program to } \\
\text { support PMI }\end{array}$ & 1 & 8 & 3 & 43 & 0 & 0 & 0 & 0 & 7 & 78 & 9 & 82 \\
\hline Perceived Police Changes & 0 & 0 & 1 & 14 & 9 & 64 & 5 & 83 & 0 & 0 & 0 & 0 \\
\hline Police mandate completion of EDP form & 0 & 0 & 1 & 14 & 9 & 64 & 4 & 67 & 0 & 0 & 0 & 0 \\
\hline $\begin{array}{r}\text { Police increased communication between frontline } \\
\text { officers and supervisors }\end{array}$ & 0 & 0 & 0 & 0 & 2 & 14 & 4 & 67 & 0 & 0 & 0 & 0 \\
\hline
\end{tabular}

